\title{
The role of bHLH transcription factor NEX in neuronal differentiation and experience-dependent plasticity
}

\author{
Dissertation \\ zur Erlangung des mathematisch-naturwissenschaftlichen Doktorgrades \\ "Doctor rerum naturalium" \\ der Georg-August-Universität Göttingen
}

vorgelegt von

Olga S. Mikhailova

aus Sankt-Petersburg (Russland)

Göttingen, 2007 
Anleiter:

Referent :

Korreferent :
Prof. Dr. Klaus-Armin Nave

Prof. Dr. Nils Brose

Prof. Dr. Kerstin Krieglstein

Tag der mündlichen Prüfung: 15. 01. 2008 
Diese Arbeit wurde im Labor von Prof. Dr. Klaus-Armin Nave am MaxPlanck-Institut für Experimentelle Medizin, Göttingen ausgeführt. 


\section{Acknowledgements}

First of all, I would like to thank Prof. Dr. Klaus-Armin Nave for giving me the opportunity of working in his group and for his scientific and moral support that was extremely important for my motivation during this study. I am deeply grateful to him for showing constant interest in my project and for giving advices that helped me to overcome the difficulties that appeared during the course of this study. I am deeply grateful to Dr. Markus Schwab for his technical and intellectual input that had significantly influenced my thesis project. His punctilious supervision and constant attention to details helped me develop scientific style in experiment design and in writing. My special thanks belong to the students and faculty members of the graduate programme "Centre for Systems Neuroscience" (ZNV). I would like to thank in particular the members of my PhD committee Prof. Krieglstein and Prof. Brose for their genuine concern and invaluable scientific advice. I would also like to thank Prof. Heinrich, Prof. Treue and Prof. Wimmer for agreeing to be members of my thesis defence committee.

The work presented in this thesis could not be possible without the immense technical support from several people. I would like to express my gratitude to Sven and Carolin for their assistance in the microarray analysis, Patricia and Ulli for various technical support and advice, Annette for help with histology and immunocytochemistry, Simone and Monika for assistance in the production of the transgenic animals, Harry, Hajo and Rolf for computer hard- and software management, Martin, Ina and Gabriele for their help with administrative issues, Markus and Eray for the construction of behavioural test setups. My biggest thanks belong to Tanja Freerk, Tanja Leinert, Sabrina, Daniel and Steffi for their excellent animal management and help they have provided me with at all times during the course of my $\mathrm{PhD}$.

I appreciate all the support, critics and intellectual guidance I have received from Frank, Sandra, Ingo, Victor, Zina, Ursula, Moritz and Toby; their advice was always fresh and useful. The organization of behaviour and memory tests, reported in this thesis, was largely based on the advice from Magda, Kostik and Prof. Giese, whose contribution made it possible to reveal specific memory deficits in the mutants. I would like to thank Chris, Maike, Viki, Lisa, Jojo, Micha, Magda, Anna and Foteini, who not only provided me with constant technical, intellectual and emotional support, but also created a warm and friendly atmosphere in the laboratory. I am grateful to all members of dept. Neurogenetics for creating an excellent working environment.

My very special gratitude and appreciation belong to Ahmet for his constant attention, advice and support in all areas of my scientific and personal life. Finally, I would like to thank my parents for giving me education. The invaluable help, support and advice they have provided me with during my whole life made all my achievements possible. 
To my parents 


\section{TABLE OF CONTENTS}

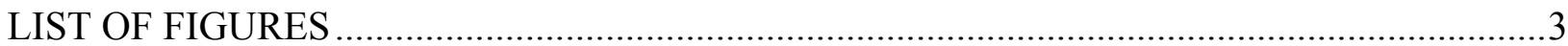

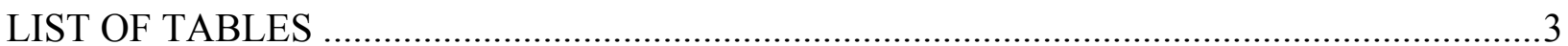

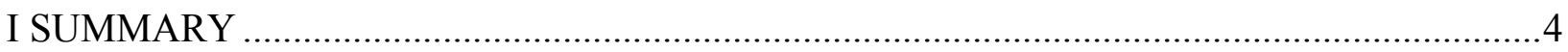

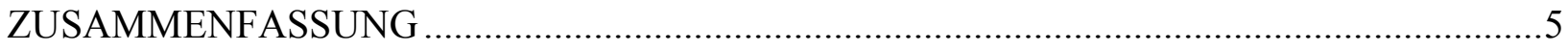

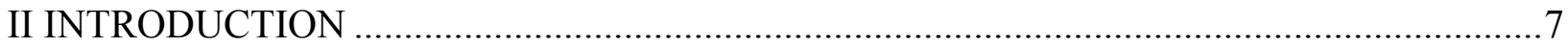

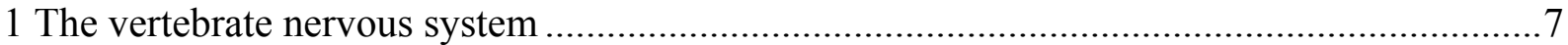

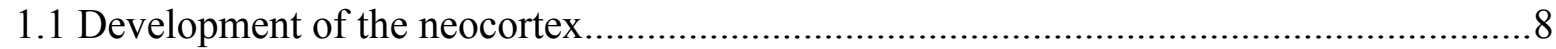

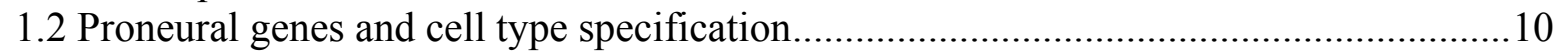

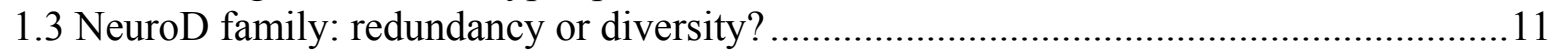

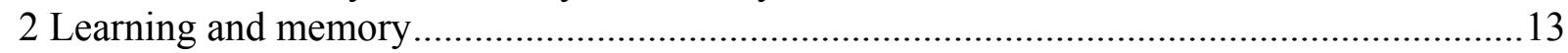

2.1 Brain areas involved in memory formation and maintenance...................................... 13

2.2 Physiological and molecular mechanisms underlying memory formation .....................15

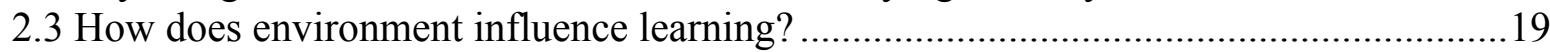

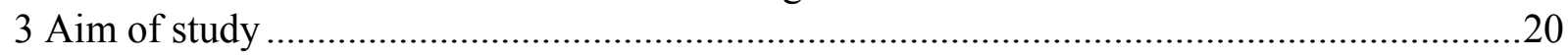

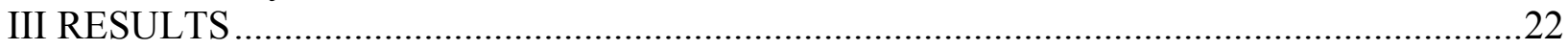

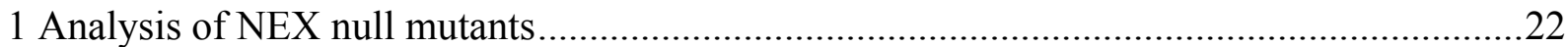

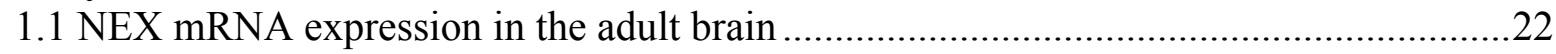

1.2 Microarray analysis of the CA3 region in NEX null mutants ......................................23

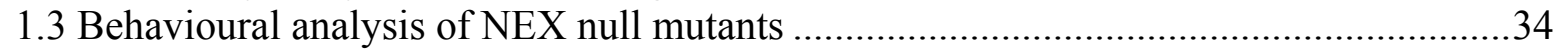

2 Cre recombinase-mediated overexpression of NEX in neural progenitor cells of the Emx 1

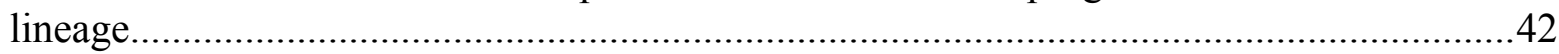

2.1 Production of a mouse line with inducible overexpression of NEX ….........................42

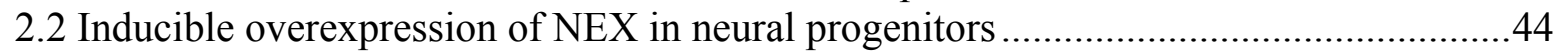

3 Generation and analysis of NEX-htTA transgenic mouse line ........................................50

3.1 BAC-based transgene construction and verification of transgene integrity ...................50

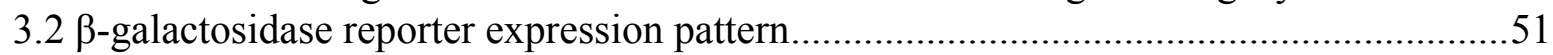

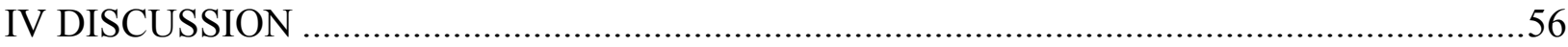

1 The effect of NEX deficiency on hippocampal gene expression in the adult mouse.............56

1.1 Expression profiling of the CA3 region of the adult hippocampus ..............................57

1.2 Gene expression changes in wildtype mice upon environmental enrichment................58

1.3 Metabolic changes in NEX null mutants indicate hyperactivity ...................................59

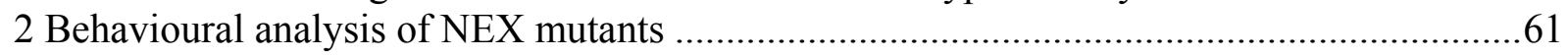

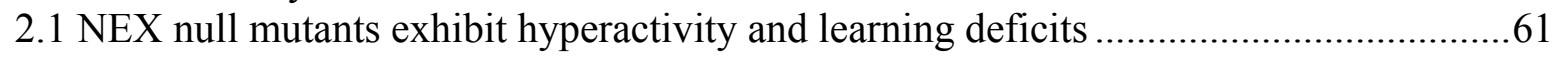

2.2 NEX deficiency has differential effects on recent and remote memory........................63

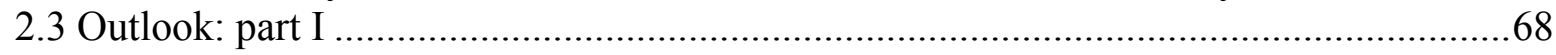

3 The effect of NEX overexpression on telencephalon development ….................................68

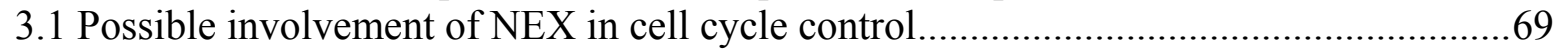

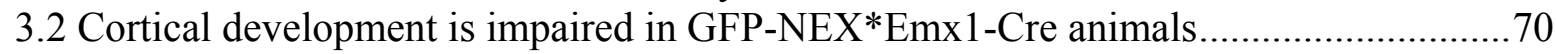

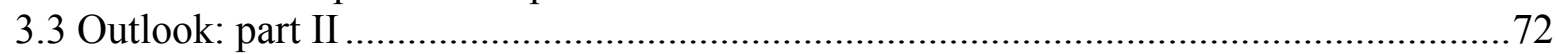

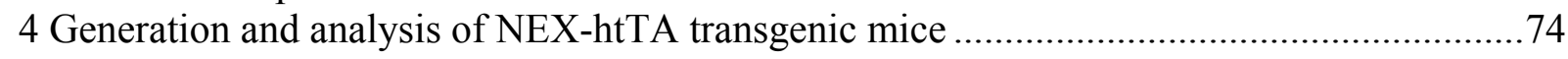

4.1 NEX-htTA transgene-mediated reporter gene expression.........................................74

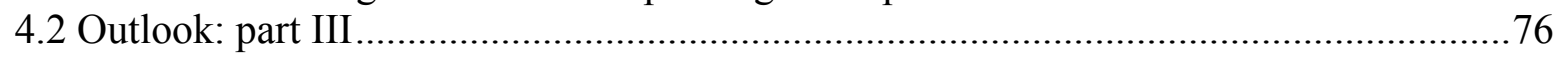

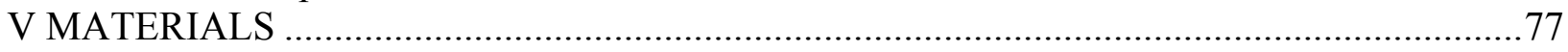

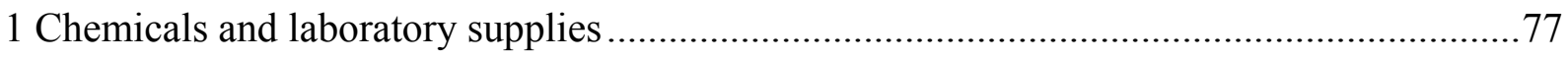

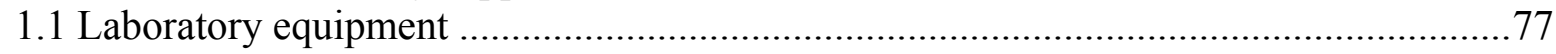

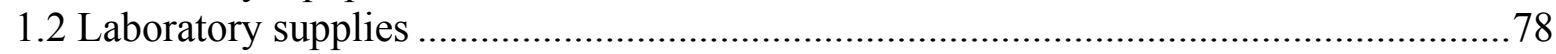

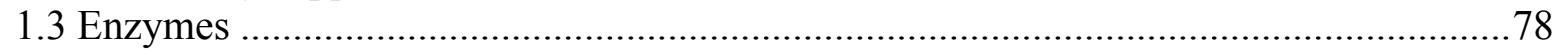

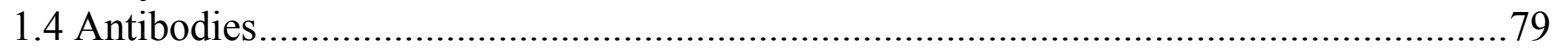

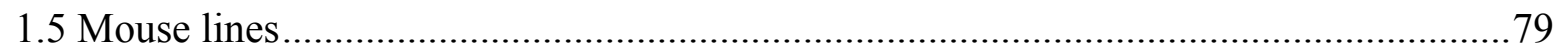




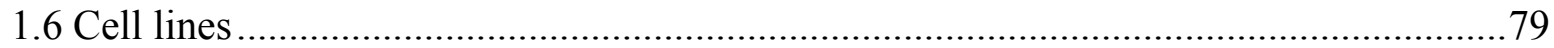

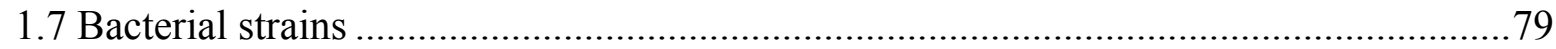

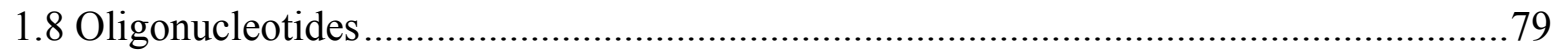

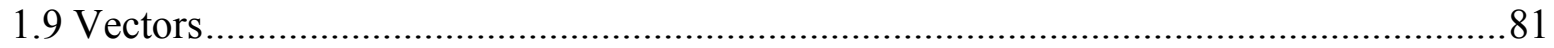

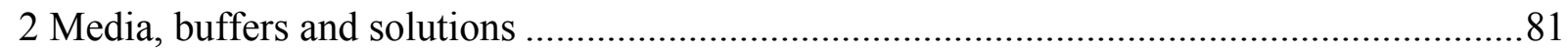

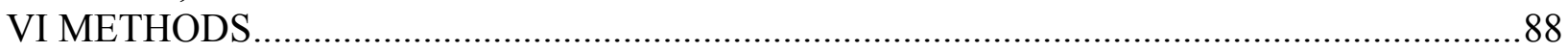

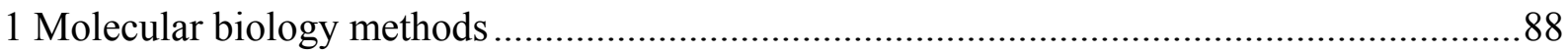

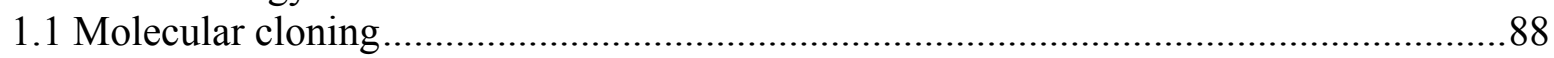

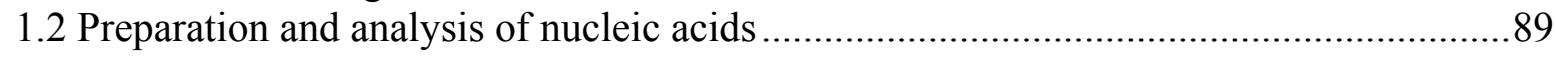

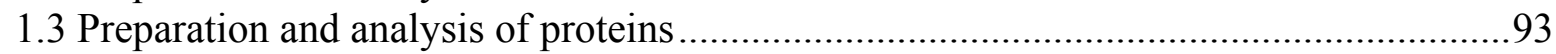

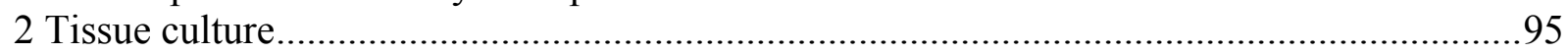

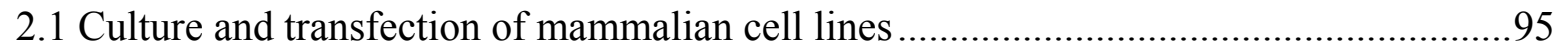

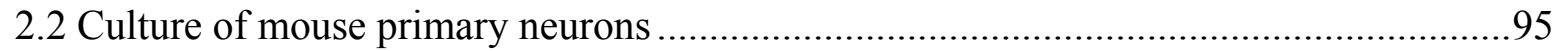

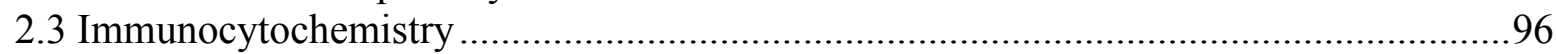

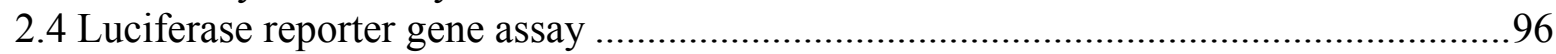

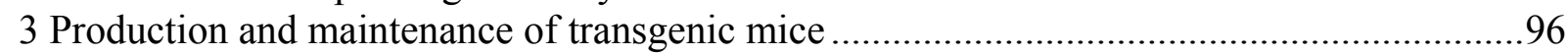

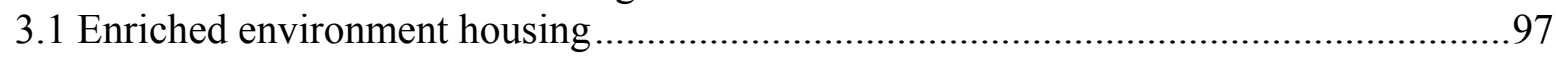

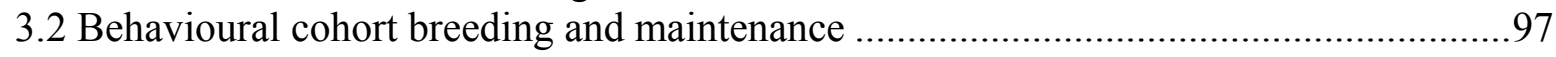

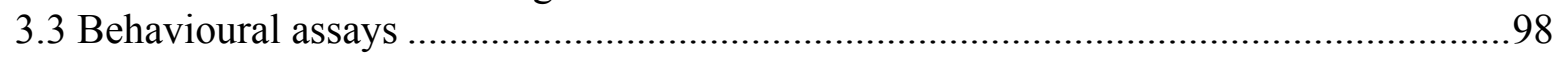

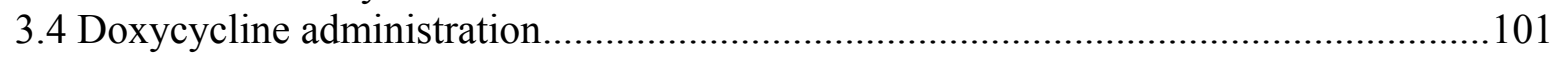

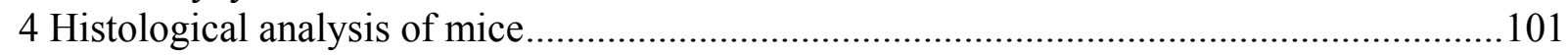

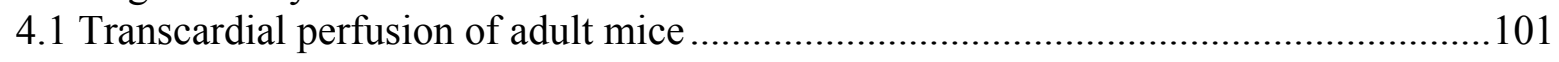

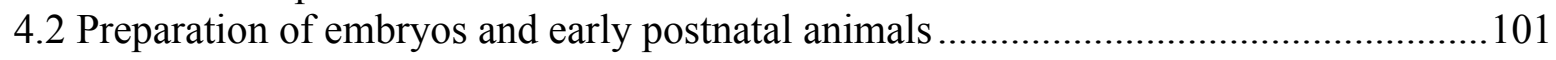

4.3 Material processing for paraffin embedding and sectioning ..................................... 102

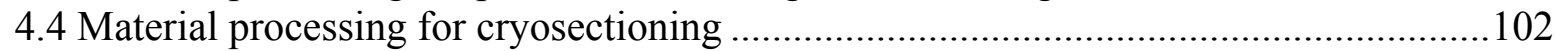

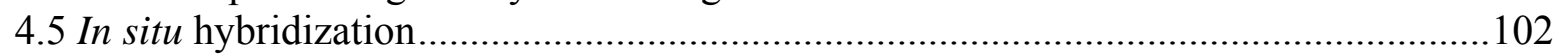

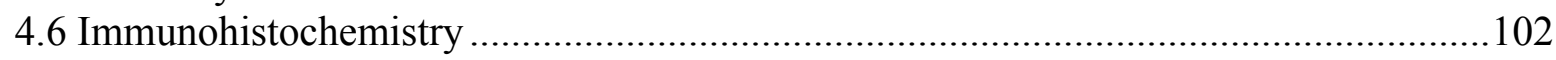

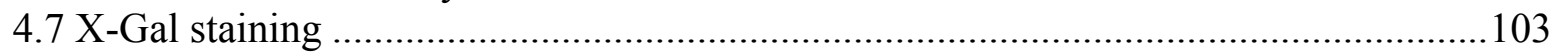

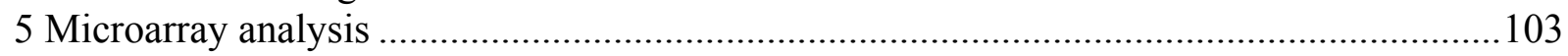

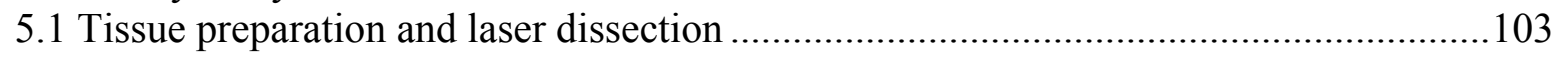

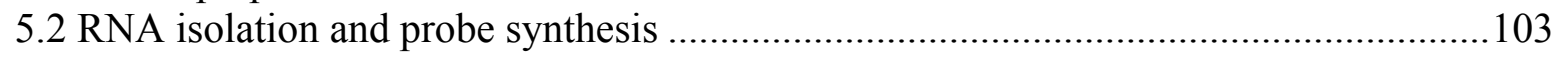

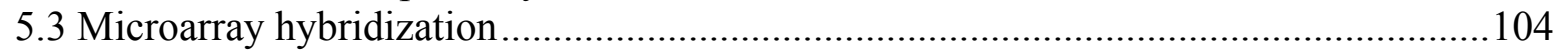

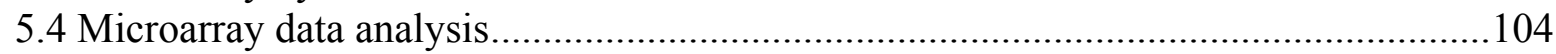

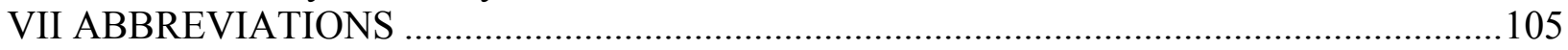

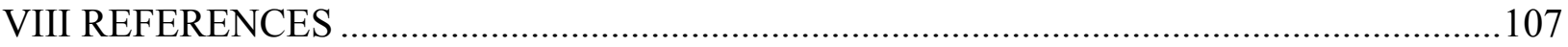

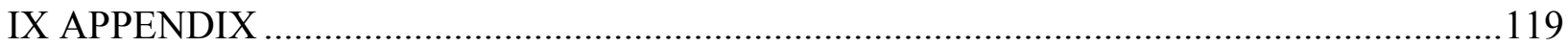

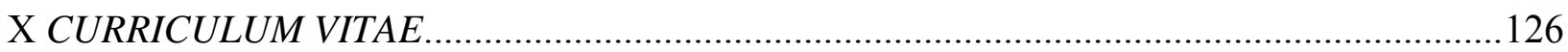

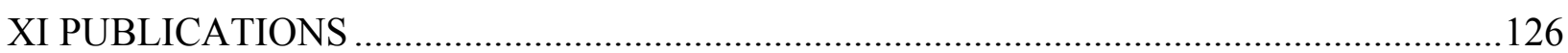




\section{LIST OF FIGURES}

Fig. 1 NEX is expressed in the CA1-3 hippocampal region of the adult hippocampus..............23

Fig. 2 Enriched environment and microarray analysis: experimental outline ............................25

Fig. 3 Enriched environment-mediated transcriptional changes in wildtype animals: gene set enrichment analysis

Fig. 4 Differentially expressed genes in the CA3 region of NEX null mutants versus wildtype controls (regular cage housing)

Fig. 5 Differentially expressed genes in the CA3 region of NEX null mutants versus wildtype controls ( 2 and 7 days in the enriched environment).....

Fig. 6 Differential gene expression in NEX null mutants and wildtype animals upon

environmental enrichment: comparison of microarray and QRT-PCR data ...............................33

Fig. 7 General physical fitness is unaltered in NEX null mutants...........................................35

Fig. 8 NEX mutants exhibit hyperactivity and higher velocity in the open field........................36

Fig. 9 NEX mutants show normal level of general anxiety in light-dark preference and plus

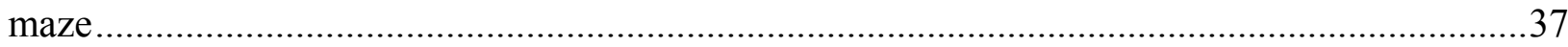

Fig. 10 NEX mutants of the first behavioural cohort show spatial learning deficits in the Morris

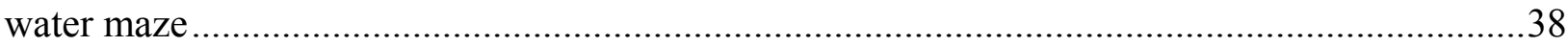

Fig. 11 NEX mutants of the second behavioural cohort show spatial learning deficits in the

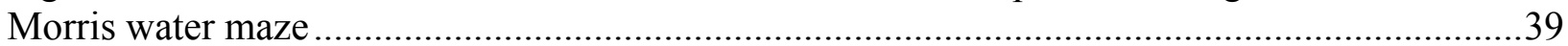

Fig. 12 The effect of different training paradigms on performance in the Morris water maze ....40

Fig. 13 Recent and remote fear memory extinction in NEX mutants ...................................41

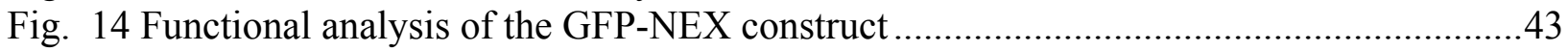

Fig. 15 Cre-mediated recombination in GFP-NEX transgenic mice........................................44

Fig. 16 Reduction of cerebral hemisphere size in GFP-NEX*Emx1-Cre transgenic mice..........46

Fig. 17 Cortical size reduction in GFP-NEX*Emx1-Cre transgenic mice.................................47

Fig. 18 Abnormal cortical layer formation in GFP-NEX*Emx1-Cre transgenic mice ...............47

Fig. 19 Increased cell death and ectopic neuronal differentiation in GFP-NEX*Emx1-Cre

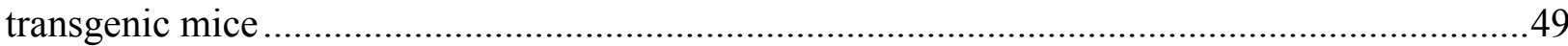

Fig. 20 Schematic representation of NEX-htTA homologous recombination; analysis of

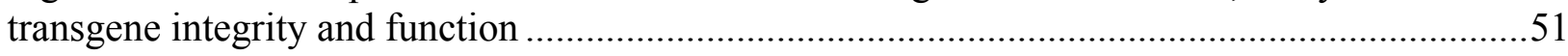

Fig. 21 NEX-htTA mediated lacZ reporter expression in embryonic development ..................52

Fig. $22 \mathrm{LacZ}$ reporter expression in the brain of adult NEX-htTA*tetO-Cre*Rosa26 mice .......53

Fig. 23 NEX-htTA*tetO-Cre*Rosa26 transgenic mice reporter expression is restricted to

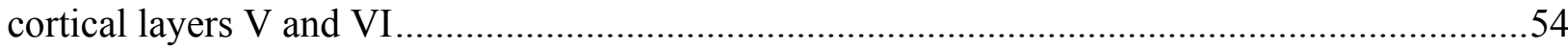

Fig. 24 htTA drives reporter expression only in a subset of pyramidal neurons in the postnatal brain

\section{LIST OF TABLES}

Tab. 1 Number of animals used for microarray analysis.

Tab. 2 Enrichment scores of gene sets that are differentially regulated upon environmental enrichment

Tab. 3 Genes that are differentially regulated in NEX null mutants versus wildtype animals in the regular cage housing.

Tab. 4 Genes that are differentially regulated in NEX null mutants versus wildtype animals after

2 days of exposure to the enriched environment.

Tab. 5 Genes that are differentially regulated in NEX null mutants versus wildtype animals after

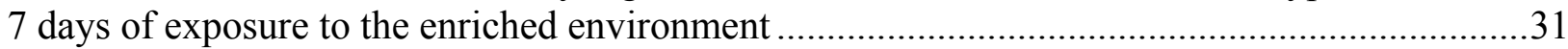

Tab. 6 X-gal staining of different reporter lines, crossed with NEX-htTA transgenic line .........55 


\section{SUMMARY}

NEX (neuronal helix-loop-helix protein) is an atonal-related basic helix-loop-helix (bHLH) transcription factor that belongs to the subfamily of neuronal differentiation factors, which also includes NeuroD and NDRF. NEX is expressed in postmitotic pyramidal neurons throughout CNS development and in the adult brain. In rodents, expression starts around embryonic day 11 in postmitotic pyramidal neurons of the cortical plate. In the adult mouse brain expression is maintained in hippocampus proper, amygdala, entorhinal cortex and subiculum. Recent in vitro studies have suggested that NEX promotes neurite outgrowth and regeneration and facilitates neuronal survival. Since inactivation of the Nex gene per se does not have an obvious impact on forebrain formation in the mouse, it is likely that the closely related proteins NeuroD and NDRF can compensate for the loss of NEX protein. However, sustained NEX expression in adult pyramidal neurons of several brain areas, which are implicated in memory formation and retrieval, suggests a function of NEX in neuronal plasticity and higher cognitive functions. The goal of this study was to investigate the function of NEX during murine telencephalon development and in experience-dependent plasticity of the adult brain.

To study the role of NEX in the adult brain, a microarray analysis of the hippocampal CA3 region of NEX null mutants versus wildtype animals was performed. In order to test the effect of the NEX null mutation on experience-induced plasticity, an enriched environment paradigm was applied prior to transcriptome analysis. The comparison of gene expression profiles of null mutant and control animals revealed a differential regulation of metabolic pathways and several genes involved in learning and memory. In line with increased metabolic activity of hippocampal neurons, NEX null mutants exhibited hyperactivity in the open field, light-dark preference and plus maze test. Further behavioural studies demonstrated a learning delay in spatial learning tasks and impaired contextual memory formation in NEX null mutants.

The spatiotemporal expression pattern of NEX during development and altered expression of cell cycle genes in null mutants suggested a role for NEX in cell cycle exit control and neuronal differentiation. To study the role of NEX during early cortical development a transgenic mouse line was generated, in which NEX expression can be induced by Crerecombinase. NEX overexpression in neuronal progenitors, but not in postmitotic neurons, resulted in the reduction of cerebral hemisphere size and abnormal cortical layer formation. Massive apoptosis of ventricular zone progenitors and ectopic expression of neuronal differentiation markers indicated premature cell cycle exit and impaired migration. 
Additionally, a transgenic mouse line allowing doxycycline-regulated control of gene expression in postmitotic pyramidal neurons was created during this study. The NEX-htTA transgenic line is currently being used to study mechanisms of cortical lamination.

\section{ZUSAMMENFASSUNG}

Das basische Helix-Loop-Helix-Protein NEX bildet zusammen mit NeuroD und NDRF eine Subfamilie neuronaler Transkriptionsfaktoren, die mit dem proneuralen Gen atonal aus Drosophila verwandt sind. Innerhalb des Vorderhirns der Maus wird NEX ausschließlich in postmitotischen Pyramidenzellen exprimiert. Die Expression von NEX beginnt am elften Embryonaltag in der Kortikalplatte und bleibt im adulten Gehirn unter anderem in der Amygdala, dem Hippokampus, dem entorhinalen Kortex und dem Subikulum erhalten. Das adulte Expressionsmuster deutet darauf hin, dass NEX an der Regulation neuronaler Plastizitätsmechanismen beteiligt sein könnte, die für den Erwerb und das Wiederabrufen von Gedächtnisinhalten notwendig sind. Verschiedene Zellkulturstudien legen nahe, dass Vertreter der NeuroD-Subfamilie am Auswachsen und der Regeneration von Axonen beteiligt sind. Da die Gehirnentwicklung in Mäusen mit einer Nullmutation des NEX-Gens weitgehend normal verläuft, ist dessen genaue in vivo-Funktion unbekannt. Im Rahmen dieser Doktorarbeit sollte die Auswirkung der Nullmutation des NEX-Gens auf das Transkriptionsprofil des Hippokampus sowie auf Lern- und Gedächtnisleistungen ermittelt werden. Daneben sollte durch Überexpression von NEX in einer transgenen Mauslinie dessen Funktion während der Kortexentwicklung untersucht werden.

Das vollständige Expressionsprofil der CA3-Region des Hippokampus von NEXNullmutanten und wildtypischen Mäusen wurde mit Hilfe einer DNA-Microarray-Analyse ermittelt. Um neuronale Plastizitätsprozesse zu verstärken, wurden NEX-Mutanten und Kontrollmäuse zuvor einer komplexen Käfigumgebung ausgesetzt. Im Rahmen der Transkriptomanalyse wurde in der Nullmutante eine erhöhte Expression von Genen des Energiestoffwechsels beobachtet. Außerdem lag eine transkriptionelle Dysregulation einer Reihe von Genen vor, die an Lern- und Gedächtnisprozessen beteiligt sind. Die erhöhte metabolische Aktivität hippokampaler Neurone korreliert mit einer Änderung des Verhaltens der NEX Nullmutanten, die im Open Field-, Light-Dark Preference- und dem Plus Maze-Verhaltenstest Hyperaktivität aufweisen. Daneben wurde eine Verlangsamung des räumlichen Lernens und Defekte in der kontextabhängigen Gedächtnisbildung beobachtet.

Das zeitliche und räumliche Expressionsmuster von NEX und die veränderte Expression von Zellzyklus-Genen in NEX-Nullmutanten weisen darauf hin, dass NEX während der neuronalen Differenzierung den Austritt aus dem Zellzyklus vermittelt. Um die Funktion von 
NEX in der Entwicklung des zerebralen Kortex zu untersuchen, wurde eine transgene Mauslinie hergestellt, die eine durch Cre Rekombinase induzierbare Überexpression von NEX ermöglicht. Die Überexpression von NEX in neuralen Vorläuferzellen, nicht jedoch in postmitotisschen Pyramidenzellen, führt zu einer deutlichen Verkleinerung des Neokortex, von der insbesondere die Schichte V und VI des Neokortex betroffen ist. Auf zellulärer Ebene wurden innerhalb der Ventrikularzone eine erhöhte Apoptoserate und eine ektopische Expression von neuronalen Differenzierungsmarkern beobachtet. Dies deutet daraufhin, dass die Überexpression von NEX in neuralen Vorläuferzellen zu einem vorzeitigen Austritt aus dem Zyklus und einer verfrühten neuronalen Differenzierung führen kann.

In einem unabhängigen Projekt wurde eine weitere transgene Mauslinie hergestellt, in der eine humanisierte Variante des Tetrazyklin-abhängigen Transaktivators (htTA) unter Kontrolle regulatorischer Sequenzen des NEX-Gens exprimiert wird. Diese Mauslinie soll zur Untersuchung von Mechanismen der Kortexentwiklung verwendet werden. 


\section{INTRODUCTION}

\section{The vertebrate nervous system}

The complexity of the nervous system is reflected by its cellular diversity as well as the variety of functions it performs. The general function of the brain is to receive input from sensory organs and, after a process of information integration and association, to transform this input into behavioural output (motor activity). The nervous system is divided into central and peripheral nervous system. The peripheral nervous system (PNS) comprises the somatic and autonomic nervous system. It receives sensory information from the body and the environment, sends signals to the spinal cord and the brain and executes motor commands of the brain. The central nervous system (CNS) consists of six major parts: spinal cord, medulla oblongata, pons and cerebellum, midbrain, diencephalon and the cerebral hemispheres. The CNS controls skeletal muscle activity and governs body homeostasis. Moreover, it is the origin of higher cognitive functions, such as learning and memory, which play an essential role in survival by regulating defence mechanisms, spatial navigation and finding of appropriate food resources (Kandel et al., 2000).

The cerebral cortex underwent the most peculiar changes during vertebrate evolution. In fish the cerebral hemispheres perform mostly olfactory function. In amphibians and reptiles the paleopallium and corpus striatum execute instinctive behaviour, whereas the archipallium is responsible for emotions. The most evolutionary developed reptiles acquire the first neurons of what will become the neocortex, and their archipallium gives rise to the hippocampal formation (Gilbert, 2006). Evolution of mammals, especially primates, is tightly connected to the development of an increasingly complex neocortex. Indeed, the number of cortical neurons in the mouse is around 4 million; this number raises significantly in carnivors and sea mammals and reaches 6,2 billion in the chimpanzee and around 11,5 billion in man (Roth and Dicke, 2005).

The basic functioning unit of the nervous system is the neuron, which is typically composed of three parts: the cell body or soma, the dendritic tree and the axon. Neurons can be classified according to their shape (uni-, bi- and multipolar), function (afferent, efferent and interneurons; excitatory and inhibitory neurons) and biochemical properties (e.g. by the type of neurotransmitter). Most of the excitatory neurons in the brain are glutamatergic, whereas the excitatory spinal motor neurons that synapse to the muscle are cholinergic. Inhibitory neurons typically use glycin or GABA as neurotransmitter. Certain unique neuronal types can be identified by their peculiar shape and location in the nervous system, e.g. Purkinje cells of the cerebellum, basket cells, pyramidal cells etc. The basic unit of neuronal activity, the action 
potential, is generated close to the cell body, propagates along the axon and is transmitted to the muscle or to another neuron through chemical or electrical synapses in a process known as synaptic transmission. Proper neuronal function would not be possible without glia, or the nonneuronal cells of the nervous system. Axonal insulation facilitates action potential propagation and nerve conduction velocity (Kandel et al., 2006). In the CNS, myelin sheaths are formed by oligodendrocytes. Astrocytes perform a variety of functions in the CNS; they enwrap synaptic connections of neurons, mediate messenger uptake and neuroactive substances release and thus participate in synaptic activity modulation (Slezak and Pfrieger, 2003; He and Sun, 2007).

This enormous cell type diversity is the result of several coordinated developmental processes that include neuroectoderm induction, proliferation, neuronal fate specification, differentiation, migration, maturation and axonal growth and guidance.

\subsection{Development of the neocortex}

The majority of cells in the vertebrate nervous system derive from the neural plate that forms on the dorsal side of the embryo during gastrulation. The formation of the neural plate is induced by morphogenic signals from the underlying notochord, which also determine the anterior-posterior patterning of the nervous system. During neurulation the edges of the neural plate fold up and ultimately form the neural tube. The specification of neuronal precursors in the neural tube involves the process of lateral inhibition mediated by Notch-Delta signalling (Gotz and Sommer, 2005). During the dorso-ventral patterning of the spinal cord a gradient of sonic hedgehog $(s h)$ is integrated into positional information and neuronal precursor subtype specification (O’Leary and Nakagawa, 2002). The posterior region of the neural tube develops into the spinal cord, and its anterior region expands to form three primary brain vesicles: prosencephalon, mesencephalon and rhombencephalon. The rhombencephalon is further divided into myelencephalon and metencephalon, from which pons, cerebellum and medulla oblongata finally form, while the mesencephalon develops into midbrain. During development the prosencephalon separates into optic vesicles, the diencephalon that gives rise to the pineal gland, hypothalamus and thalamus, and the telencephalon that comprises the developing olfactory bulbs, hippocampus and neocortex (Gilbert, 2006; Wolpert et al., 2006).

All neurons and glia cells of the neocortex arise from a proliferative zone aligning the lumen of the neural tube, i.e. the ventricular zone. Pyramidal neurons are produced in the ventricular and subventricular zone of the dorsal telencephalon and migrate radially (within the same radial column) through the intermediate zone to the developing cortical plate along radial glia. Their neuronal fate and layer identity is specified before migration, and the earlier-born neurons occupy deeper cortical layers, whereas the later-born neurons migrate further to the 
superficial layers thus forming an inside-out gradient. Cajal-Retzius cells and GABA-ergic interneurons are produced in subpallial regions and migrate tangentially into the cortex (Gotz and Sommer, 2005).

Genetic studies have revealed a number of genes that control progenitor fate specification during neocortical development. Among those transcription factors that determine neuronal progenitor identity are Pax6, Ngn2, Emx2 and Mash1 (Hamasaki et al., 2004; Parras et al., 2002; Gotz and Sommer, 2005; Scardigli et al., 2003; Takahashi and Osumi, 2002). Expressed in well defined regions of the developing CNS, these factors instruct morphological and biochemical characteristics that determine future neuronal properties. Numerous studies have demonstrated that specification of regional identity and neurotransmitter fate is determined concomitant to or even before the determination of general neuronal identity (Sur and Rubenstein, 2005; Schuurmans and Guillemot, 2002; Schuurmans et al., 2004). However, it is still unclear whether the combination of transcription factors at a certain time point or the series of patterning and identity choices over a period of time result in the final identity of a given neuron.

Once the cortical plate is formed, functional interactions between neighbouring and distant neurons have to be established to allow networking of different brain areas. The pyramidal neurons of the neocortex establish connections to ipsi- and contralateral sides of the cortex, the thalamus and the spinal cord via axon outgrowth. Axonal pathfinding depends on both axonal identity and environmental cues. Several axon guidance molecules, such as SLIT, ROBO, semaphorins, ephrins and netrins, have been implicated in this process (Plachez and Richards, 2005; Chilton, 2006). Upon growth cone arrival at the target, initial contact triggers the recruitment of pre- and post-synaptic proteins and functional synapse establishment (Price et al., 2006).

CNS development is not limited to embryogenesis; several very important developmental and fine-tuning processes continue postnatally, e.g. neurogenesis of cortical and hippocampal interneurons (Hatten, 1999), cerebellar granule cell migration and differentiation. Myelination of axons from projection neurons in brain and spinal cord ensures axonal protection, insulation and therefore efficient action potential propagation. The establishment of visual, auditory and somatosensory circuits occurs after birth and is experience-dependent (Erzurumlu and Kind, 2001; Fox, 2002). Fine-tuning in the adult CNS involves synaptic plasticity; de novo synapse formation and modification continues throughout life and accounts for learning and memory processes. 


\subsection{Proneural genes and cell type specification}

Research in the past decades has elucidated some of the mechanisms that generate complex neuronal phenotypes. Studies in Drosophila and vertebrates have identified a group of basic helix-loop-helix (bHLH) transcription factors that are involved in both general neuronal fate specification and location-dependent identity. Several evolutionary distinct groups of bHLH transcription factors have been described that act in cascades to generate specific neuronal phenotypes throughout the CNS (Bertrand et al., 2002; Ross et al., 2003; Ma, 2006).

The bHLH domain, that gave this protein family its name, is required for heterodimerization and binding to the CANNTG consensus cis-element (E-box) in the promoter region of target genes. Most of the vertebrate bHLH genes were identified in various screens due to their sequence similarity to the Drosophila genes atonal (ato) and achaete-scute (asc) or their ability to bind the product of the Drosophila sex determination gene daughterless (da) (Chae et al., 2004). In Drosophila, members of asc and ato families are expressed in the ectoderm before any overt neuronal differentiation and are both required and sufficient for the generation of neuronal progenitor cells in PNS and CNS (Jan and Jan, 1994; Jimenez and Modolell, 1993). Gain- and loss-of-function experiments have demonstrated that these genes are similar in both their ability to induce proneural fate and the capacity to trigger the differentiation of a particular type of sensory organ when ectopically expressed (reviewed in Bertrand et al., 2002).

The vertebrate homologues of asc include ash1 (present in mouse, Xenopus and chicken) and three other genes, present only in one of the three model organisms (Mash2, Xash3 and Cash4) (Hassan and Belen, 2000; Bertrand et al., 2002). Ato homologues are represented by several subfamilies: the atonal family, which shows the closest homology to the ato bHLH domain (Math1 and Math5), the neurogenin (Ngn), the NeuroD and the Olig subfamilies (Bertrand et al., 2002). Two other distinct groups of bHLH factors are the E-proteins (including E12, E47, ME1 and ME2 - the homologues of da), that act as co-activators, and the Id family, members of which lack the basic domain and act as repressors of the other bHLH proteins (Ross et al., 2003).

The general mode of action of bHLH genes in nervous system development is to orchestrate a cascade of fate determination and differentiation genes, similar to what has been observed in muscle development (Weintraub, 1993; Jan and Jan, 1993; Lee, 1997). Neuronal fate determination genes are expressed transiently during development and trigger the expression of differentiation genes that are required for further generation of subtype diversity and neuronal survival. Neuronal differentiation factors are able to induce neuronal fate when ectopically expressed, but they depend on the transcriptional control of the true proneural genes both in 
Drosophila (Jarman et al., 1993; Goulding et al., 2000) and the mouse (Lee et al., 1995; Sommer et al., 1996; Farah et al., 2000; Hevner et al., 2006).

Target gene specificity of bHLH factors is encoded, in part, in the basic (i.e. DNA-binding) domain. Indeed, a single aminoacid in the basic domain of zebrafish NeuroD determines its ability to produce sensory neurons (Wang et al., 2002). The HLH domain swap between ato and asc in Drosophila also demonstrated that specific target gene expression, and therefore cell fate determination depends on the sequence of the basic domain (Chien et al., 1996).

While the basic domain determines binding to certain target promoters, the HLH domain mediates protein-protein interactions, which allow heterodimerization with E-proteins and recruitment of other transcription factors.

Finally, protein regions outside the bHLH domain share the least similarity within and between protein families and are therefore probably involved in the functional divergence of bHLH factors (Morgenstern and Atchley, 1999). Of particular interest are the potential phosphorylation sites in the activation domains and the plastic activity changes they might provide at different stages of development and adult life (Khoo et al., 2003; Hand et al., 2005; Martelli et al., 2006; Seet et al., 2006).

\subsection{NeuroD family: redundancy or diversity?}

NEX (neuronal helix-loop-helix protein), the first member of the NeuroD family, was cloned in a PCR-screen using degenerate primers against Drosophila bHLH genes (Bartholoma and Nave,1994; see also Shimizu et al., 1995), followed by the isolation of NeuroD (neuronal determination factor; Lee et al., 1995; Naya et al., 1995) and NDRF (NeuroD- related factor; Yasunami et al., 1996). NeuroD, NEX and NDRF share approximately 93\% homology within the bHLH domain. NeuroD family members display $51 \%$ homology to the bHLH domain of atonal. All three factors are capable of inducing neuronal differentiation in cultured cells (Farah et al., 2000; Cho et al., 2001; Uittenbogaard and Chiaramello, 2002; Oda et al., 2005) and NeuroD and NDRF have been shown to trigger ectopic neuronal differentiation upon overexpression in Xenopus ectoderm (Lee et al., 1995; McCormick et al., 1996). However, since embryonic expression of these proteins is largely restricted to committed cells outside the ventricular zone and was shown to depend on the expression of neurogenins, these factors act downstream from neuronal determination genes (Ma et al., 1996; Fode et al., 1998; Ma et al., 1999; Lin et al., 2004). In addition to neuronal differentiation, NeuroD is involved in the development of pancreatic beta-cells (Mutoh et al., 1997). Mutations of the human homologue of NeuroD have been shown to cause Type 1 and 2 diabetes mellitus (Malecki et al., 1999), and 
NeuroD overexpression under the insulin promoter in null mutant mice (Miyata et al., 1999) rescues the pancreatic phenotype.

Mice lacking NeuroD in the CNS exhibit a loss of dentate gyrus and cerebellar granule cells and a subset of inner ear sensory ganglia, resulting in ataxia and deafness (Miyata et al., 1999; Schwab et al., 2000; Liu et al., 2000; Kim et al., 2001). NDRF null mutants show normal development until two weeks after birth, but develop ataxia afterwards and die between P14 and P23 (Olson et al., 2001). The mutants also exhibit a thinner cerebellar granule cell layer, downregulation of neurotrophic factors in the cerebellum (Olson et al., 2001), impaired amygdala development (Lin et al., 2005) and defects in thalamocortical axon terminal segregation in the somatosensory cortex (Ince-Dunn et al., 2006). In contrast, no obvious histological abnormalities have been reported in NEX null mutants (Schwab et al., 1998). A comparative expression analysis revealed that all three family members possess overlapping expression domains in the developing CNS (Schwab et al., 1998). This observation and high sequence similarity led to the hypothesis that NeuroD, NEX and NDRF perform redundant functions in differentiating forebrain neurons (Ross et al., 2003). Indeed, dentate gyrus formation is more affected in the absence of both NEX and NeuroD when compared to NeuroD single null mutants (Schwab et al., 2000). Interestingly, although all three factors show abundant expression in pyramidal neurons of the neocortex and the hippocampus, no gross deficits in differentiation or survival of these cells have been reported in single gene loss-of-function experiments (Kume et al., 1998; Schwab et al., 1998; Chae et al., 2004; Cho and Tsai, 2004).

The expression of neuronal bHLH proteins is maintained in the adult telencephalon (Schwab et al., 1998; Goebbels et al., 2006). High levels of NeuroD and NDRF mRNA are detected in the granule cell layer of the cerebellum, hippocampal CA1-3 areas and dentate gyrus. NEX is virtually absent from the cerebellum, but expressed at high levels in the CA1-3 region of the hippocampus, entorhinal cortex and subiculum (Schwab et al., 1998). The three factors show different expression patterns in the amygdala, where NEX and NDRF, but not NeuroD, are expressed in the basolateral anterior amygdaloid nucleus (Goebbels, 2002; Lin et al., 2005). NEX mRNA levels predominate those of the other two factors in the CA3 region of the hippocampus, the entorhinal cortex and the subiculum (Schwab et al., 1998; M. Gummert, unpublished observation), i.e. areas essential for the functional connection between hippocampus and neocortex (de la Prida et al., 2006; Yasuda and Mayford, 2006).

Persistent expression suggests that these factors are required for the maintenance of pyramidal neurons and neuronal plasticity in the adult brain. Indeed, since neuronal bHLH factors drive synaptic and cytoskeletal protein expression in cell culture and in development (Chiaramello et al., 1996; Oda et al., 2005; Munoz et al., 2007), these factors might be used later 
in life for replenishing the dendritic and axonal protein pools and plasticity-dependent changes. Recent in vitro studies identified NEX target genes (Uittenbogaard and Chiaramello, 2005), which indicate that NEX might be involved in the maintenance of adult pyramidal neurons by promoting neuronal survival. NeuroD, in turn, has been implicated in experience-dependent adult neurogenesis (Deisseroth et al., 2004). Finally, NDRF heterozygous mutants show emotional learning deficits (Lin et al., 2005). Taken together, expression patterns and loss-offunction studies suggest that, in addition to a certain degree of functional redundancy between the family members, evolutionary pressure has created a diversity of functions of neuronal bHLH proteins during development as well as in the survival and plasticity of mature neurons.

\section{Learning and memory}

\subsection{Brain areas involved in memory formation and maintenance}

"We are what we remember." Eric Kandel

Learning and memory play an important role in a wide range of phenomena, from simple conditioning, present in virtually all multicellular organisms, to higher cognitive functions in humans. The current classification divides memories into declarative, or explicit, and nondeclarative, or implicit. Importantly, it has been shown that in contrast to explicit memory the recall of implicit memory is always unconscious. In fact, implicit memory formation is found even in the primitive forms of invertebrates, and its molecular mechanisms have been successfully studied in Aplysia (reviewed in Kandel, 2006; see also Barco et al., 2006).

In humans the various types of explicit memory can be divided into episodic (memory of events and locations) and semantic (general knowledge of facts) memory. Case studies in humans revealed the importance of the medial temporal lobe (MTL), a brain region that includes hippocampus, entorhinal cortex, peri- and postrhinal cortex, subiculum and pre- and parasubiculum, for memory formation and storage (Squire and Zola-Morgan, 1991; Squire, 1992; Eichenbaum, 2004; Squire et al., 2004). Subjects with MTL lesions (one of the most famous cases was that of patient H.M., published by Scoville and Milner in 1957) suffer from anterograde amnesia. However, the damage affects mostly the formation of new declarative memories, while other forms of mnemonic processing (visuomotor tasks, perceptual learning and repetition priming) are largely spared. The degree of retrograde amnesia can vary greatly between patients, but in general episodic memories are more susceptible to temporally graded retrograde amnesia, whereas some semantic memories are spared if the damage does not exceed the MTL (Frankland and Bontempi, 2005). These observations suggest the presence of several anatomically distinct memory systems. 
Explicit memory formation is connected to higher cognitive function and has been extensively studied in the mouse and other mammals. In the following chapters emphasis will be put on the spatial and emotional learning in rodents. The key player in spatial learning is the hippocampus, which enables navigation in familiar and non-familiar environments and is believed to serve as a cognitive map. So called "place cells" in the CA1 region of the hippocampus "record" certain body positions in the surrounding and allow later recollection of the specific path the animal has followed (Skaggs and McNaughton, 1996). The path recording is often recalled during sleep, when certain CA1 neurons repeat the firing pattern they have performed while the animal was navigating.

Observations made in human subjects and animals have led to a system-based model of consolidation, according to which the hippocampus rapidly stores events before the information is transferred to the cortex for subsequent sorting and reorganization (Marr, 1970; Marr, 1971). According to the current system consolidation theory, experience is encoded in parallel in cortex and hippocampus, and the subsequent reactivation of hippocampus (e.g. during sleep) leads to the strengthening of hippocampo-cortical and cortico-cortical connections and gradual transfer of the memory to cortical networks (Squire and Alvarez, 1995). However, to avoid "overloading" the brain with unnecessary information the path and spatial cues are only recorded when associated with a positive or negative stimulus. It can be the reward at the end of the maze or an electric shock that allows for the memory of a specific environment to be consolidated in association with the stimulus. Therefore the hippocampus actively interacts with other brain areas, e.g. prefrontal and anterior cingulate cortex, amygdala, striatum and entorhinal cortex, to ensure that only relevant memory is stored.

In rodents, one of the most widely accepted spatial memory tasks is navigation in the Morris water maze (Morris, 1981), which is based on rats and mice being naturally good swimmers, yet expressing a strong motivation to escape from water. Generally the animals are trained over a period of 1-2 weeks to find a hidden escape platform in a round pool filled with opaque liquid by using spatial cues on the walls surrounding the pool (Crawley, 2000). Classical Pavlovian conditioning is used in rodents to evaluate emotional learning and explicit memory of a particular location. The most common paradigm includes positioning the animal into a novel environment (conditioned stimulus I) and presenting it with a tone (conditioned stimulus II), followed by a mild foot shock (Fanselow, 1980; LeDoux, 1995). The memory of both cage and tone are later tested by evaluating the level of "freezing" (lack of all motor activity except breathing), the specific fear response rodents demonstrate when the conditioned stimulus is presented again (Crawley, 2000). Freezing is a common response in many species and is thought to be an evolutionary advantageous mechanism, as an unmoving animal is less noticed by a 
predator (LeDoux, 1996). Importantly, studies of explicit memory usually include implicit components: thus, mice have to learn the motor reflex of swimming in the Morris water maze or the freezing reflex during fear conditioning in addition to learning the specific spatial locations; and in some instances the animal re-links a previously learned motor task with novel environmental cues (McGaugh, 2005; Rossato et al., 2006).

The multitrace nature of memories presents a challenge for the further investigation of cognitive processes and suggests replacing studies of single candidate genes or inactivation of single brain areas by a system approach, based on the analysis of microarray data and the interactions of several brain areas.

\subsection{Physiological and molecular mechanisms underlying memory formation}

\subsubsection{LTP and LTD}

The hippocampal formation harbours three excitatory inputs: (I) the perforant path, which connects the subiculum to the dentate gyrus (DG), (II) the mossy fibres, running from the DG to the pyramidal cells in the CA3 region, and (III) the Schaffer collaterals from CA3 to CA1 neurons. In 1973 Bliss and Lomo demonstrated that a high-frequency stimulation of any of these three pathways can produce a potentiation in postsynaptic hippocampal neurons, which can last for hours or even days. This phenomenon was called long-term potentiation (LTP) (Bliss and Lomo, 1973). LTP has different properties in CA3 and CA1 neurons, being non-associative in the former. In contrast, LTP in CA1 pyramidal cells is characterized by cooperativity, associativity and specificity. This and the persistent nature of LTP have led to the hypothesis that it is closely associated with memory formation (Bliss and Collingridge, 1993).

Another form of neuronal plasticity, long-term depression (LTD), was originally induced by low-frequency stimulation in CA1 hippocampal neurons (Lynch et al., 1977). It is now clear that LTD can be induced in virtually all excitatory synapses throughout the CNS by a variety of mechanisms. Importantly, induction of LTD as well as LTP depends greatly on the recent history of synaptic activity (Malenka and Bear, 2004); for example, prior tetanization can inhibit subsequent LTP (Abraham and Hugget, 1997) and facilitate LTD (Holland and Wagner, 1998). The temporal persistence of LTP and LTD is linked to the intensity of NMDA receptor activation and protein synthesis. Nguyen and colleagues (1994) have postulated the existence of a temporal window after LTP induction, during which the protein synthesis-independent LTP (or early LTP) can be consolidated by recruitment of plasticity-associated proteins. Their findings supported the development of the synaptic tag hypothesis (Frey and Morris, 1997; Frey and Morris, 1998), that suggests targeted sequestering of plasticity proteins to specific synapses and 
their contribution to consolidation in an input-specific manner (for review, see Martin et al., 2000).

Whether LTP and LTD are indeed the cellular mechanisms of memory remains an open question, although a number of studies have shown a clear link between LTP and memory impairment in mouse mutants (Giese et al., 1998; Mansuy et al., 1998; Winder et al., 1998). Moreover, the induction of LTP in the hippocampus following experience in a living animal has been demonstrated recently (Whitlock et al., 2006), and the molecular mechanisms involved in LTP and LTD formation and maintenance give us reasons to believe that neurons use long-term potentiation and depression at least as one of the tools for memory recording.

\subsubsection{The molecular signatures of short-term and long-term memory}

Memories can be classified as short-term or long-lasting, the latter sometimes persevering throughout life. In the mouse, short-term memory of an object, specific environment or event is limited to 2-3 hours, whereas long-term memory is established during 24 hours and can last for months or even years (Kandel, 2001). If we consider long-term potentiation or depression in the hippocampus to be the basic physiological event of memory formation, the varying molecular signatures of these processes should to some extent reflect the varying nature of memories. Indeed, it has been shown that early and late LTP differ not only in the time they persist, but also in molecular properties, in the sense that late LTP requires protein synthesis (Malenka and Bear, 2004).

Short-term memory is believed to exist purely at the local synapse. After the potentiating stimulus has occurred synaptic strengthening is triggered by glutamate receptors that stabilize spine motility by decreasing actin turnover (Dillon and Goda, 2005) and recruiting profilin, Ncadherin, $\beta$-catenin, $\alpha \mathrm{N}$-catenin and contractin to the postsynaptic region (Ackermann and Matus, 2003; Bozdagi et al., 2000; Murase et al., 2002; Abe et al., 2004; Hering and Sheng, 2003). The hypothesis of a "synaptic tag" suggests that it also captures plasticity-related proteins that are later used to further stabilize synaptic strength. The current model of spontaneous synaptic plasticity suggests a balance of kinase-phosphatase activity at the synapse, which is shifted to one side in the process of synapse stabilization (Dillon and Goda, 2005). Interestingly, while LTP induction and maintenance depend on protein phosphorylation, LTD was shown to be protein-phosphatase 1 (PP1)-dependent (Hu et al., 2007).

Eric Kandel has described the process of memory storage as "a dialogue between genes and synapses" (Kandel, 2006). Unlike short-term memory, which is characterized only by synapse strengthening, long-term memory requires de novo transcription and protein synthesis, as originally shown in Aplysia (Kandel, 2001). In the sensitization paradigm, elevated levels of 
cAMP and protein kinase A (PKA), when combined with continuous stimulation, led to phosphorylation and activation of MAPK and the transcription factor CREB, translocation of which to the nucleus is necessary for long-term memory formation in several models (Impey et al., 1998). NMDA and AMPA receptors were also shown to play a major role in this signalling cascade. They act through activation of CaMKII (calcium-calmodulin dependent kinase II), which could serve as a molecular switch for memory storage at the active synapse (Lisman et al., 2002). CaMKIV can also be activated in the MEK-MAPK cascade and is able to phosphorylate CREB (Roberson et al., 1999). Several other growth factors (e.g. BDNF) and transcription factors (such as jun, fos and Zif-268) have been implicated in LTP and memory formation (reviewed in Johnston et al., 2003). Studies of several mouse mutants have confirmed the importance of signalling cascades (e.g. PKA, PKC, CaMKII and MEK cascades) in long-term memory formation (Shalin et al., 2004; Fleishmann et al., 2003; Angelo et al., 2006; Isiegas et al., 2006).

Studies of sensitization in Aplysia and of formation of explicit memory in rodent hippocampus have revealed that implicit and explicit memory formation exploits essentially the same molecular mechanisms at the synapse (Barco et al., 2006). Intracellular signalling from the postsynapse to the nucleus results in the activation of nuclear transcription factor CREB, which facilitates target gene expression. Interestingly, many CREB target genes, such as c-fos, are also transcription factors. They often belong to the class of immediate early genes, and their ability to be activated in response to synaptic activity and to activate the transcription of downstream genes is believed to account for long-term memory formation. The de novo synthesised gene products are captured at the active synapse, thus ensuring the strengthening and growth of existing connections and probably facilitating the activation of pre-existing silent synapses and new synapse formation (reviewed in Barco et al., 2006).

\subsubsection{Reconsolidation and extinction of long-term memory}

Memories are not carved in stone, but rather written in ink. Recollection of apparently fully consolidated memories initiates a process of reconsolidation and, under some circumstances, extinction (Nader, 2003). Several studies have demonstrated the loss of memory during retrieval in the presence of inhibitors of transcription (reviewed in Tronson and Taylor, 2007); moreover, retrieval-induced reconsolidation was shown to differ from original consolidation on the molecular level (Lee et al., 2004). The well established paradigm of fear memory extinction demonstrates that even strong fear memory in rodents can be modified, and it is not clear whether traces of the original memory survive the extinction process. Extinction is currently viewed as de novo memory formation, and although both reconsolidation and extinction of 
memory are transcription-dependent, they have distinct temporal and biochemical characteristics (Suzuki et al., 2004).

Long-term memory can be further classified into recent and remote. These terms not only reflect the temporal aspect, but also the brain area where the memory is stored. Recent studies of human subjects and mouse mutants suggest that spatial memories, though formed in hippocampus, are not permanently stored there (Squire and Bayley, 2007). Accordingly, posttraining hippocampal lesions disrupt recent, but not remote memories (Maviel et al., 2004). One of the current models of system consolidation views hippocampus as a temporary depository, where memories are formed and replayed before being transferred to the cortex (reviewed in Frankland and Bontempi, 2005). Studies of brain activity during sleep have revealed that reactivation of memory trace not only occurs in hippocampus (Skaggs and McNaughton, 1996), but also in hippocampo-cortical and cortico-cortical networks (Sutherland and McNaughton, 2000; Wilson, 2002; McNaughton et al., 2006). In support of the memory transfer hypothesis, rats with disrupted cortico-hippocampal connections (lesion in the afferent input from layer III of the entorhinal cortex, the so-called temporoammonic projection) show normal functioning of hippocampus but fail at a 28-day retention memory test (Remondes and Schuman, 2004). CaMKII heterozygous mutant mice show normal learning and memory when tested 1-3 days post training, but are severely impaired when tested 10-50 days after training, - a time period, during which memory would have become dependent on the cortex (Frankland et al., 2001; Frankland et al., 2004). A similar pattern of memory loss was observed in mice, when a dominant-negative form of p21-activated kinase was overexpressed in cortical neurons (Hayashi et al., 2004). In these mutants remote memory deficits were associated with impaired cortical plasticity. Because of the difference in recent and remote memory storage, different brain areas (and possibly different molecular mechanisms) could be involved in the respective extinction processes. Indeed, inhibition of protein synthesis in the hippocampus facilitates recent memory extinction, but has no effect on remote memory (Frankland et al., 2006).

Behavioural experiments and studies of molecular mechanisms reveal a high conservation of memory formation-dependent changes at synapses and in nuclei of neurons in different brain areas as well as in different species. At the same time, evidence is growing that the connections and cross-talk between different brain areas underlie many cognitive processes of mammals. Therefore, a systems approach has to be applied to fully understand the formation and maintenance of memory. 


\subsection{How does environment influence learning?}

Experimental rodents are typically housed in a low complexity environment, which generally includes food and water provided ad libitum and sometimes nesting material. Thus, the phenotype of many mouse mutants is left unnoticed, since this caging provides no challenge for proper brain function. On the other hand, some mouse mutants with impaired learning in the laboratory setup show flexible behaviour and improve their learning abilities in a more naturalistic environment (Vyssotsky et al., 2002). In some cases enriched environment provides a positive "challenge", as revealed by studies of animal models of various human cognitive disorders and trauma. Environmental enrichment or even physical activity have been shown to improve cognitive and histological symptoms in mouse models of Hungtington's, Alzheimer's and Parkinson's diseases, ALS, epilepsy, stroke and traumatic injury (reviewed in Nithianantharajah and Hannan, 2006).

The enriched environment paradigm was first described by Donald Hebb in 1947 (cited after Nithianantharajah and Hannan, 2006). Enriched cages used nowadays typically contain running wheels, simple maze-like structures and objects of various colour and texture, which stimulate motor, cognitive, visual and somatosensory brain functions. If the animals are housed in the cage for a long time period, occasional changing of objects or their location provides additional enrichment. An enriched environment has a variety of effects on wildtype rodents: it has been shown to increase dendritic branching and length, number and size of synapses in certain populations of neurons, hippocampal neurogenesis and functional integration of the newborn neurons (reviewed in van Praag et al., 2000). On the molecular level, it enhances the levels of BDNF, NGF, various synaptic proteins, NMDA and AMPA receptors and other proteins implicated in synaptic plasticity (Rampon et al., 2000), and, not surprisingly, results in increased synaptic strength and LTP (Foster et al., 1996; Foster and Dumas, 2001). At the behavioural level, environmental enrichment enhances exploratory activity and decreases anxiety, furthermore, it has been shown to facilitate learning and memory (Schrijver et al., 2002; Lee et al., 2003) and to rescue memory decline in senile rodents (Kobayashi et al., 2002).

Several studies have used microarrays to analyse gene expression changes induced by environmental enrichment in cortex and hippocampus (Rampon et al., 2000; Tong et al., 2001; Molteni et al., 2002). Interestingly, both physical exercise and environmental enrichment result in upregulation of CREB, CaMKII, PKC and other genes involved in memory formation. These data point to a tight link between environment, experience-dependent plasticity and learning. Further analysis of the molecular mechanisms involved in plasticity changes will greatly help to understand cognitive processes and assist in the development of therapies for human cognitive disorders. 


\section{Aim of study}

\section{Aim 1: Determining the effect of the NEX null mutation on hippocampal expression profile, learning and memory}

NEX (neuronal helix-loop-helix protein) belongs to the NeuroD family of atonal-related bHLH transcription factors and is expressed in postmitotic pyramidal neurons throughout CNS development and in adulthood. Cell culture studies have shown that NEX facilitates neuronal survival and promotes neurite outgrowth and regeneration in PC12 cells.

In the adult murine brain, expression is maintained in hippocampus proper, amygdala, entorhinal cortex and subiculum. While in developing forebrain the expression domains of NEX and the related factors NeuroD and NDRF largely overlap, in the adult brain NEX mRNA levels dominate in CA3 region of the hippocampus, entorhinal cortex and subiculum. Its unique expression pattern and the observation that NEX is a putative CREB target gene (Uittenbogaard et al., 2007) implicate a role for NEX in neuronal plasticity, memory formation and retrieval. However, the null mutant mice generated earlier (Schwab et al., 1998) show normal development and no obvious histological defects in the adult brain. A systemic approach is required to link a potential role of NEX in synaptic plasticity changes induced by environmental enrichment to hippocampal-dependent learning and subsequent memory transfer to other brain areas. Therefore, the aim of this study was to determine the effect of a NEX null mutation on mouse behaviour and to analyse the transcriptional changes in the CA3 region of the hippocampus in NEX null mutants compared to wildtype mice upon environmental enrichment.

\section{Aim 2: Generation of a transgenic mouse line that permits inducible overexpression of NEX}

Studies of mouse mutants provide valuable information about the loss of function of the gene of interest. However, in case of functional redundancy, particular deficits might be masked by the compensatory action of related factors. The lack of an obvious developmental phenotype in NEX null mutants might be due to a functional redundancy, since NeuroD and NDRF, two structurally related bHLH transcriptional factors, have overlapping expression domains in the developing mouse telencephalon (Schwab et al., 1998). Overexpression studies often prove useful in revealing the specific function of closely related genes; therefore, a gain-of-function approach was used in this study to investigate the role of NEX in mouse brain development. The consequence of Cre-recombinase mediated overexpression of NEX in early progenitors was studied with the help of Emx1-Cre mouse line (Gorski et al., 2002). Investigation of the role of 
increased dosage of NEX in later stages of neuronal differentiation and in the adult brain will be performed with the help of NEX-Cre transgenic line (Goebbels et al., 2006).

Additionally, a mouse transgenic line, allowing doxycycline-regulated control of gene expression in pyramidal neurons, was created within this study. The NEX-htTA transgenic line will be used for inducible transgene expression in the developing cortical neurons and studies of cortical lamination mechanisms. 


\section{RESULTS}

\section{Analysis of NEX null mutants}

The neuronal bHLH transcription factor NEX has been previously shown to trigger neuronal differentiation in cultured cells and to facilitate expression of genes involved in neuronal survival and plasticity (Uittenbogaard and Chiaramello, 2004; Uittenbogaard and Chiaramello, 2005). Together with the fact that NEX is expressed in brain areas that are involved in memory formation this suggests a role for NEX in neuronal maintenance and higher cognitive functions. NEX null mutants exhibit no obvious developmental deficits; they have a normal life span and cage behaviour. A detailed molecular and behavioural analysis was performed to determine the function of NEX in adult neuronal plasticity. A microarray approach was chosen as a means of high-throughput screening of transcriptional changes. To further increase the sensitivity and precision of the analysis, it was combined with laser-capture microdissection of specific brain areas (Rossner et al., 2006). Additionally, enriched environment, which is known to trigger plasticity changes and facilitate neuronal survival and metabolic activity and therefore acts as a form of positive cognitive challenge, was provided for experimental animals.

\subsection{NEX mRNA expression in the adult brain}

High levels of postnatal NEX expression have been shown in the hippocampus, entorhinal cortex and subiculum (Schwab et al., 1998). In this study additional expression analysis was performed to assess NEX expression level in the hippocampus of adult wildtype mice. To determine the expression level of NEX mRNA in the adult mouse brain, in situ hybridization with a DIG-labelled NEX-specific cRNA probe was performed on coronal cryosections of wildtype male mice at different ages. High levels of NEX mRNA were detected in the CA1-3 region of the hippocampus in 2, 6 and 12 month old animals (Fig. 1A-C). The CA1 and CA3 hippocampal regions were chosen for microarray analysis of NEX mutants. Data obtained from the expressional profiling of the CA3 region is presented in this thesis, whereas profiling of the CA1 region is still ongoing.

Additionally, RT-PCR with NEX-specific intron-spanning primers, performed on the cDNA prepared from brain RNA extracts of NEX null mutants and wildtype controls, demonstrated the absence of NEX message from mutant brain (Fig. 1D). 


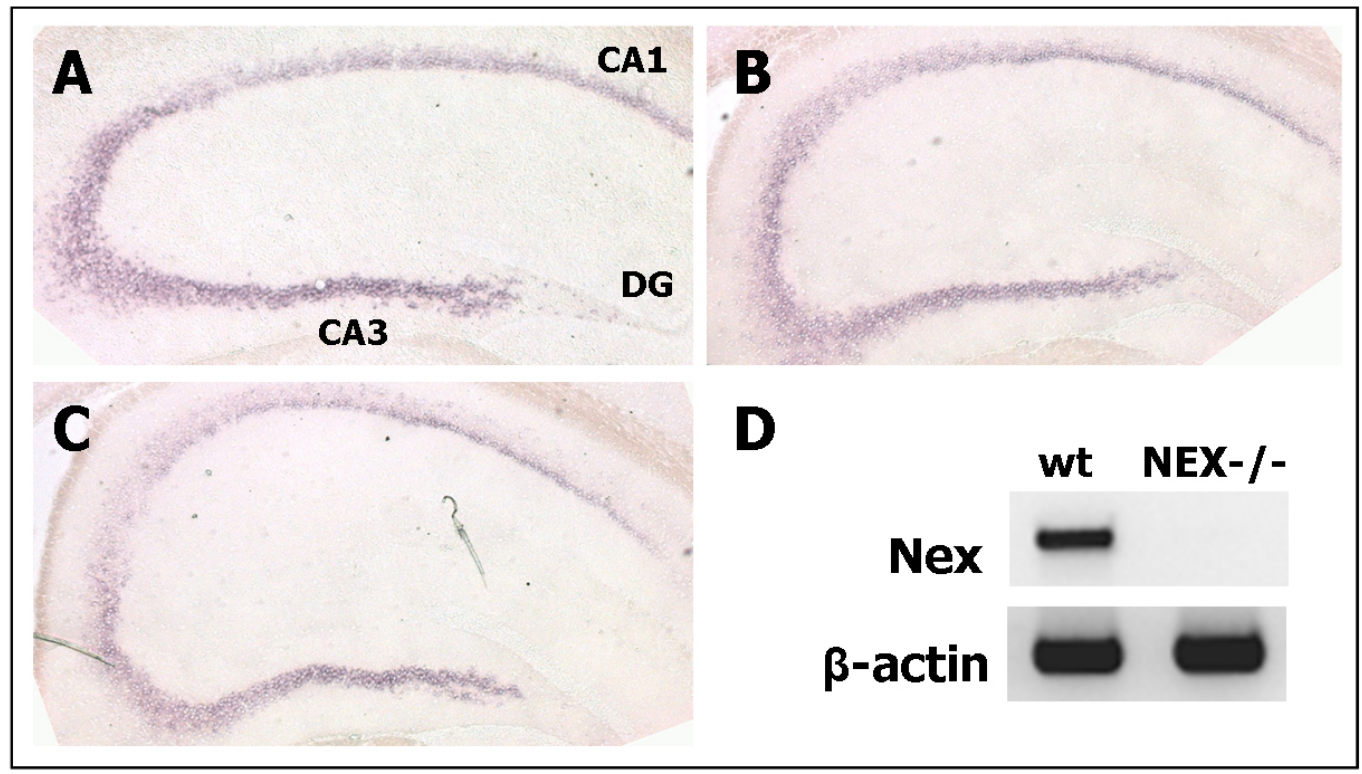

Fig. 1 NEX is expressed in the CA1-3 hippocampal region of the adult hippocampus

In situ hybridization shows expression of NEX mRNA in the CA1-3 regions of the hippocampus at 2, 6 and 12 months (A, B and $\mathbf{C}$, respectively). Note the absence of signal from dentate gyrus (DG).

(D) NEX message is absent from the brain of null mutants as demonstrated by RT-PCR.

\subsection{Microarray analysis of the CA3 region in NEX null mutants}

Several groups of animals, each containing mutant and wildtype male littermates, were housed in regular cages or exposed to an enriched environment for 2 or 7 days at the age of nine weeks. The enriched environment cages were approximately 3 times larger than conventional cages $(36 \times 50 \times 20 \mathrm{~cm})$. Each enriched environment cage was equipped with a running wheel, a 2floor house, a crunchy tunnel and a wall separating "living area" from "food and water area" as shown in Fig. 2A. Every day an inner carton of a toilet paper roll and a brightly coloured novel object were placed into the cage. Additional enrichment was provided by handling of the mice. Thus all criteria of environmental enrichment were met: novelty, physical exercise, cognitive, visual and somatosensory stimulation (Nithianantharajah and Hannan, 2006). The total number of animals, exposed to different levels of environmental enrichment, is presented in Tab. 1. Prominent transcriptional changes have been observed to take place already after two days of environmental enrichment (Rampon et al., 2000; Ronnback et al., 2005). In this study the duration of exposure was set for two and seven days in order to monitor the onset of expressional changes in the beginning of the positive challenge as well as at the time point when these changes are likely to acquire a steady state. 
Tab. 1 Number of animals used for microarray analysis

\begin{tabular}{|c|c|c|}
\hline $\begin{array}{c}\text { Time of exposure } \\
\text { to the enriched } \\
\text { environment }\end{array}$ & NEX-/- & wt \\
\hline $\begin{array}{c}\text { 0 d (regular cage } \\
\text { housing) }\end{array}$ & 4 & 4 \\
\hline $2 \mathrm{~d}$ & 4 & 4 \\
\hline $7 \mathrm{~d}$ & 3 & 4 \\
\hline
\end{tabular}

After exposure to the enriched environment animals were sacrificed by cervical dislocation at $1 \mathrm{pm} \pm 1$ hour. Brains were freshly dissected and immediately placed onto dry ice, wrapped in aluminium foil and parafilm and stored at $-80^{\circ} \mathrm{C}$ until sectioning. Serial coronal sections were prepared from the left hemisphere in the region of hippocampus (bregma -1,7 to 2,0). After laser-capture microdissection (LCM) of several CA3 regions (shown in Fig. 2B-D), the samples were pooled within groups and total RNA isolation and probe synthesis were performed according to a recently established protocol (Rossner et al., 2006). Briefly, the frozen samples were rapidly thawed at RT, half of each sample was transferred into a fresh tube, and RNA isolation was performed as described in the Methods section. The eluted RNA samples were pooled within groups (same genotype, same duration of exposure to the enriched environment). RNA was precipitated and subsequently resuspended with T7-tagged oligonucleotides. Two rounds of T7 RNA polymerase-mediated RNA amplification and biotin labelling were performed, after which probe quality was analyzed with the Agilent 2100 Bioanalyzer.

Hybridization, washing, staining, and scanning were performed under standard conditions as described by the manufacturer. Mouse 4302.0 gene chips were used that contain over 22,600 probe sets representing transcripts and variants from over 14,000 mouse genes. Two technical replicas were used for each sample. The average correlation coefficient was 0,993 for each pair of replicas (see Fig. 2E). 


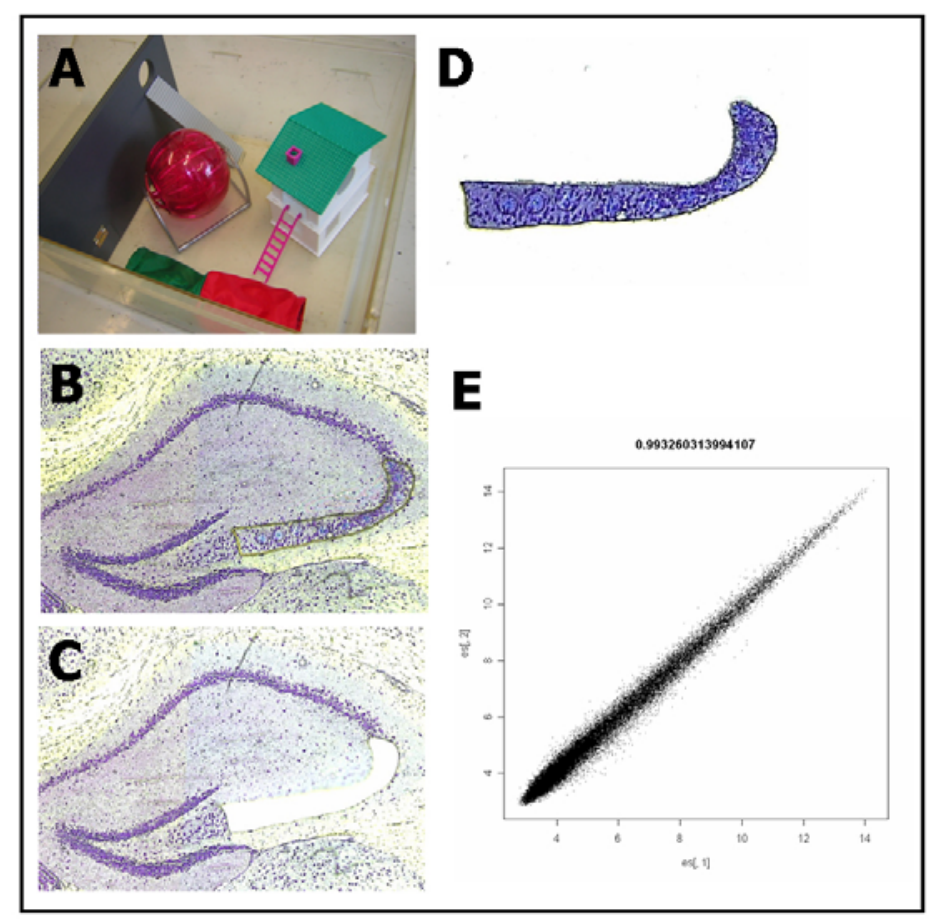

Fig. 2 Enriched environment and microarray analysis: experimental outline

Mutant and wildtype males were exposed to the enriched environment cage (A) for 2 or 7 days at 9 weeks of age (control animals were housed in regular cages). Brains were dissected and fresh-frozen on dry ice. CA3 regions were isolated from $20 \mu \mathrm{m}$ thick thionin-stained cryosections (B-D) and stored in RLT buffer. Three samples per group were pooled prior to RNA extraction and amplification. After two rounds of amplification and quality check the material was hybridized to Affymetrix GeneChip ${ }^{\circledR}$ Mouse Genome 4302.0 Arrays. Two technical replicas were used for each sample. The average correlation coefficient between replicas was 0,993. (E) Example of scatter plot for two technical replicas.

Microarray hybridization data was first normalized within the same arrays and between different arrays with the help of software from the affy package and limma package (Smyth et al., 2005; both packages are available from the Bioconductor Platform; www.bioconductor.org). Significance analysis of microarrays (SAM) was performed with the corresponding SAMPackage also available from Bioconductor. A gene set enrichment analysis (GSEA; Mootha et al., 2003; Subramanian et al., 2005) was performed on pairwise comparisons; data obtained from animals of different genotypes or different duration of exposure to the enriched environment were compared.

\subsubsection{Effect of environmental enrichment on gene expression in the $\mathrm{CA} 3$ region}

The analysis of enriched environment-induced changes of gene expression was first performed for wildtype mice (C57B16N background) in order to assess the level of metabolic and plasticity changes the used paradigm induced in the CA3 region. Here, a gene set enrichment analysis demonstrated an increase in neuronal metabolism and total activity (Fig. 3). 


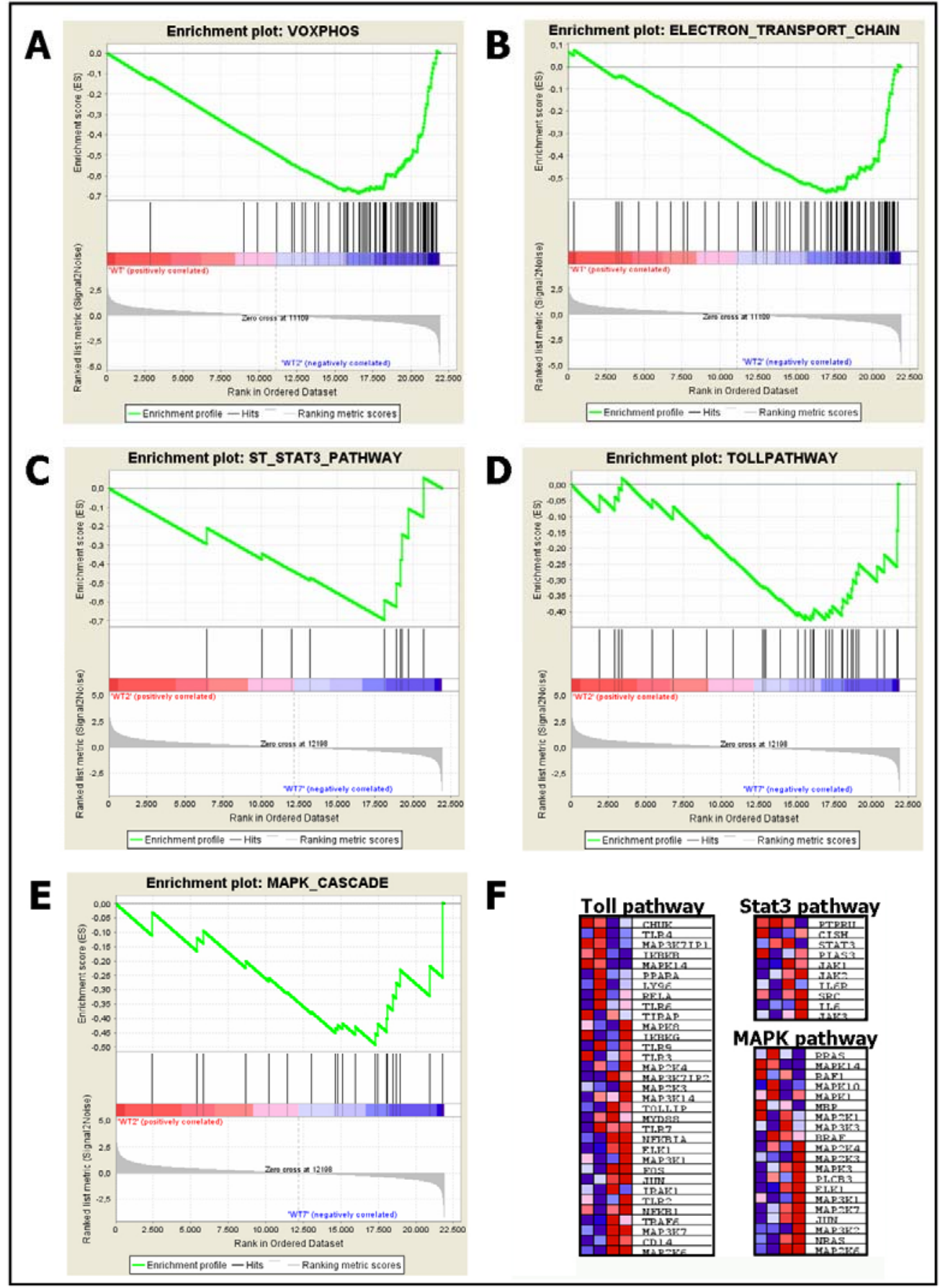

Fig. 3 Enriched environment-mediated transcriptional changes in wildtype animals: gene set enrichment analysis

Oxidative phosphorylation (A) and electron transport chain (B) gene sets are upregulated in wildtype mice that were housed in the enriched environment for two days, when compared to regular cage controls. The gene sets of Stat3, Toll and MAPK (C-E) pathways are upregulated in animals housed in the enriched environment for 7 days, when compared to those exposed to enriched environment for 2 days. (F) Heat maps of the members of gene sets presented in C-E.

A comparison of animals from regular cages with those that were exposed to the enriched environment for 2 days revealed an activation of genes involved in oxidative phosphorylation and the electron transport chain upon environmental enrichment, indicating an increase in metabolic activity in the CA3 region. More pronounced changes in neuronal activity occurred after a 7 day exposure to the enriched environment (Fig. 3C-E): the expression of components of 
the Stat3, Toll and MAPK pathways were significantly elevated when compared to a 2 day exposure (see heat maps in Fig. 3F). The enrichment scores and p values of obtained for the gene sets mentioned above are presented in Tab. 2.

Tab. 2 Enrichment scores of gene sets that are differentially regulated upon environmental enrichment

\begin{tabular}{|l|c|c|c|}
\hline \multicolumn{1}{|c|}{ Gene set } & $\begin{array}{c}\text { Enrichment score } \\
\text { (ES) }\end{array}$ & Normalized ES & p value \\
\hline Oxidative phosphorylation & $-0,69$ & $-2,67$ & $<0,001$ \\
\hline Electron transport chain & $-0,56$ & $-2,2$ & $<0,001$ \\
\hline Stat3 pathway & $-0,7$ & $-1,77$ & 0,026 \\
\hline Toll pathway & $-0,43$ & $-1,5$ & 0,047 \\
\hline MAPK pathway & $-0,49$ & $-1,48$ & 0,117 \\
\hline
\end{tabular}

\subsubsection{Differentially expressed genes in the CA3 region of NEX null mutants}

First, single-gene and gene set enrichment analysis was used for a comparison between NEX null mutants and wildtype mice housed in regular cages. Altogether, 39 genes were found to be significantly (i.e. expression level showed more than $50 \%$ difference between samples) downregulated and 98 genes were found to be upregulated in the CA3 region of NEX null mutants. The expression levels of NEX-related bHLH proteins NeuroD and NDRF was unaltered in NEX mutants.

A subset of differentially expressed genes is presented in Tab. 3. Identified genes were classified according to their function (transcriptional regulation, mitochondrial and ribosomal proteins, cell cycle-related genes, genes implicated in memory and behaviour, etc; see Fig. 4). The group distribution of down- and upregulated genes is shown in Fig. 4. A gene set enrichment analysis revealed shifts in metabolic activity-related pathways in mutant animals: diacylglycerol pathway, oxidative phosphorylation and electron transport chain gene sets were significantly upregulated in the null mutants (Fig. 4). 
downregulated
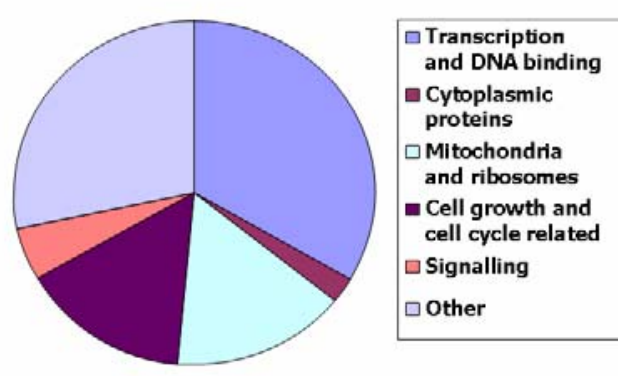

upregulated

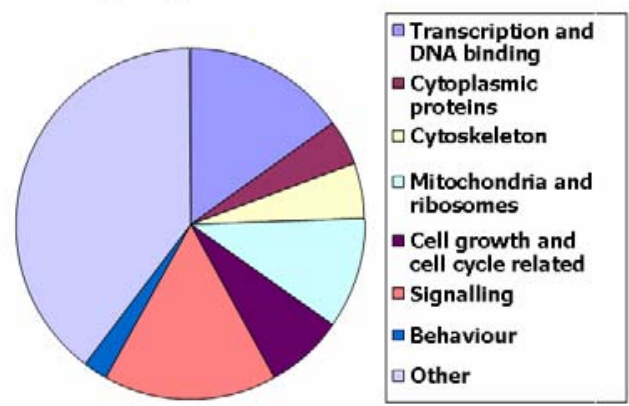

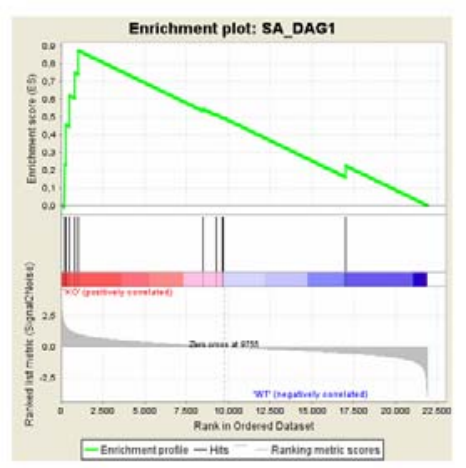
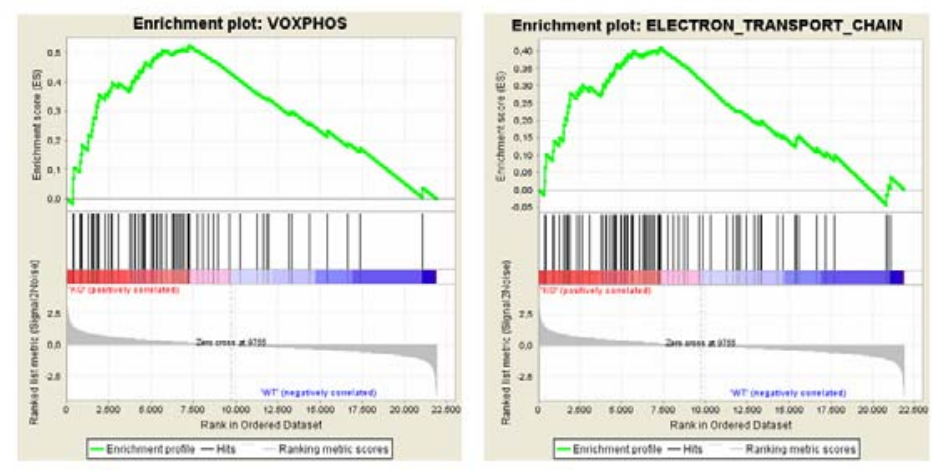

Fig. 4 Differentially expressed genes in the CA3 region of NEX null mutants versus wildtype controls (regular cage housing)

Diagrams above display the group distribution of 39 genes, which are downregulated (left), and 98 genes, which are upregulated (right), in NEX null mutants compared to wildtype controls.

The gene set enrichment plots below demonstrate upregulation of diacylglycerol pathway (left; ES 0,52; NES 2,12; $\mathrm{p}<0,001$ ), oxidative phosphorylation gene set (middle; ES 0,87; NES 2,15; $<0,001$ ) and electron transport chain gene set (right; ES 0,41; NES 1,74; $\mathrm{p}<0,001$ ) in NEX null mutants.

(ES enrichment score; NES normalized enrichment score)

Group distribution of genes, which are differentially regulated in NEX null mutants compared to wildtype animals at 2 and 7 days of environmental enrichment, is presented in Fig. 5. Approximately the same number of genes was found to be differentially expressed in regular caging, 2 days and 7 days of enriched environment exposure (137, 124 and 151, respectively). However, the group distribution was different at different time points (Fig. 4 and 5). Interestingly, genes involved in the regulation of transcription tend to be upregulated in the null mutants. The share of metabolism-controlling genes that were upregulated in NEX mutants increased upon environmental enrichment (Fig. 5). More differences in the expression level of various genes involved in cell cycle control were found after 7 day exposure to the enriched environment than at earlier time points.

A detailed list of genes with a differential expression of more than 1,5 fold between genotypes is presented in Tab. 3 (regular cage housing), Tab. 4 (2 days of environmental enrichment) and Tab. 5 (7 days of environmental enrichment). 


\section{2 days}

A downregulated

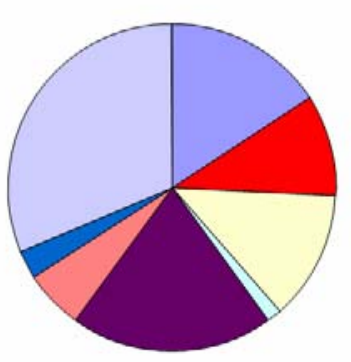

C

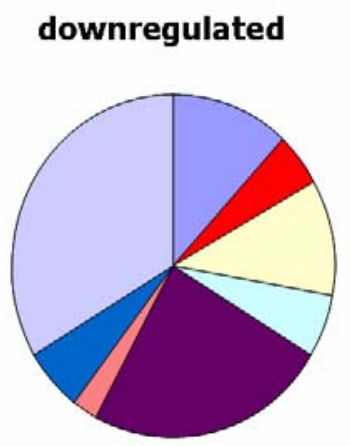

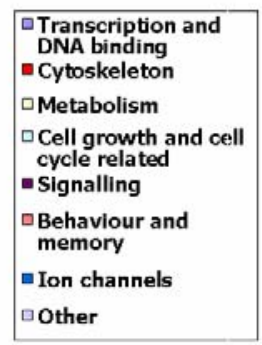

\section{7 days}

\begin{tabular}{|l|}
\hline Transcription and \\
DNA binding \\
- Cytoskeleton \\
$\square$ Metabolism \\
$\square$ Cell growth and cell \\
cycle related \\
Signalling \\
Behaviour and \\
memory \\
Synaptic proteins \\
and lon channels \\
$\square$ Other \\
\hline
\end{tabular}

B upregulated

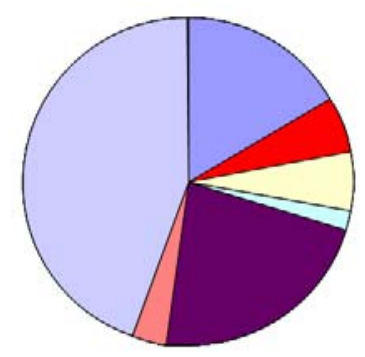

D

upregulated

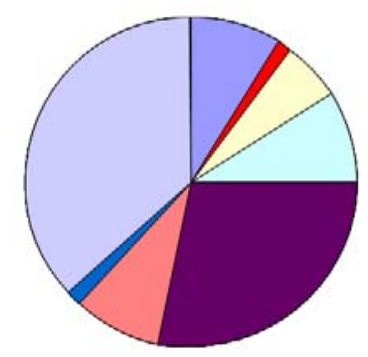

Fig. 5 Differentially expressed genes in the CA3 region of NEX null mutants versus wildtype controls (2 and 7 days in the enriched environment)

(A) Group distribution of 70 genes, which are downregulated in the NEX null mutants compared to wildtype animals after 2 days of environmental enrichment.

(B) Group distribution of 54 genes, which are is upregulated in the NEX null mutants compared to wildtype animals after 2 days of environmental enrichment.

(C) Group distribution of 83 genes, which are downregulated in the NEX null mutants compared to wildtype animals after 7 days of environmental enrichment.

(D) Group distribution of 68 genes, which are upregulated in the NEX null mutants compared to wildtype animals after 7 days of environmental enrichment.

The expression of two transcription factors, Pou3f1 and Egr2, was notably dysregulated in null mutants, housed in both regular and enriched cages. Several cytoskeleton-related genes and components of intracellular signalling pathways were differentially expressed in NEX mutants. Genes involved in behaviour and cognitive functions, such as Fos, Prkar2b, ttr and ptgds demonstrated misexpression in the null mutants at different time points (Tab. 3-4). Finally, pronounced differences in the expression of cell cycle-related genes (Cdkn1a, Nedd9 and reelin) were detected after prolonged exposure to the enriched environment. 
Tab. 3 Genes that are differentially regulated in NEX null mutants versus wildtype animals in the regular cage housing

\begin{tabular}{|c|c|c|c|c|c|}
\hline \multirow{2}{*}{\multicolumn{2}{|c|}{$\begin{array}{l}\text { Genes downregulated in } \mathrm{KO} \\
\text { Transcription }\end{array}$}} & \multicolumn{4}{|c|}{ Genes upregulated in $\mathrm{KO}$} \\
\hline & & \multicolumn{2}{|c|}{ Transcription } & \multicolumn{2}{|c|}{ Cell Growth and Cell Cycle } \\
\hline Gene Symbol & Fold reg. & Gene Symbol & Fold reg. & Gene Symbol & Fold reg. \\
\hline Egr2 & 7,66 & Pou3f1 & 15,18 & Igfbp4 & 8,58 \\
\hline Fos & 3,80 & $\mathrm{Ldb} 2$ & 2,18 & Nov & 7,67 \\
\hline Fos12 & 3,38 & Rbm39 & 2,15 & Gas5 & 2,31 \\
\hline Nfkbie & 3,01 & St18 & 2,01 & Bex1 & 1,58 \\
\hline Npas4 & 2,97 & Grhl1 & 1,99 & & \\
\hline Zfp451 & 2,68 & Lmo1 & 1,88 & & \\
\hline Irf2bp2 & 2,42 & Zfp97 & 1,83 & & \\
\hline $\mathrm{Nr} 4 \mathrm{a} 2$ & 2,03 & Ankrd10 & 1,83 & & \\
\hline \multirow[t]{2}{*}{ Nfatc2 } & 1,83 & Trps 1 & 1,75 & \multicolumn{2}{|l|}{ Synapse } \\
\hline & & Sfrs6 & 1,67 & Gene Symbol & Fold reg. \\
\hline \multicolumn{2}{|c|}{ Cell Growth and Cell Cycle } & \multicolumn{2}{|c|}{ 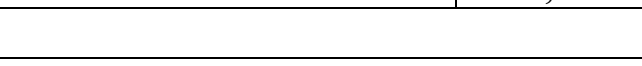 } & Doc2b & 4,38 \\
\hline Gene Symbol & Fold reg. & \multicolumn{2}{|c|}{ Actin cytoskeleton } & Stxbp6 & 2,97 \\
\hline Bid & 2,38 & Gene Symbol & Fold reg. & Arhgap20 & 2,30 \\
\hline \multirow[t]{2}{*}{ Ccnd2 } & 1,94 & Tmsb10 & 9,47 & & \\
\hline & & Hook1 & 1,88 & \multicolumn{2}{|c|}{ Mitochondrion } \\
\hline \multicolumn{2}{|l|}{ Mitochondrion } & Marcks & 1,59 & Gene Symbol & Fold reg. \\
\hline Gene Symbol & Fold reg. & & & Dsp & 9,06 \\
\hline 0610009A07Rik & 6,38 & \multicolumn{2}{|c|}{ Behaviour, learning and memory } & $\operatorname{Cox} 7 \mathrm{c}$ & 2,42 \\
\hline Arg2 & 2,48 & Gene Symbol & Fold reg. & Cyp26b1 & 2,22 \\
\hline \multicolumn{2}{|c|}{ Behaviour, learning and memory } & Calb1 & 21,10 & Tomm20 & 1,77 \\
\hline Gene Symbol & Fold reg. & Penk1 & 3,07 & Ndufs5 & 1,69 \\
\hline Plcl1 & 2,78 & Prkar2b & 2,16 & Ndufb7 & 1,58 \\
\hline
\end{tabular}


Tab. 4 Genes that are differentially regulated in NEX null mutants versus wildtype animals after 2 days of exposure to the enriched environment

\begin{tabular}{|c|c|c|c|}
\hline \multicolumn{2}{|c|}{ Genes downregulated in $\mathrm{KO}$} & \multicolumn{2}{|c|}{ Genes upregulated in $\mathrm{KO}$} \\
\hline \multicolumn{2}{|c|}{ Transcription } & \multicolumn{2}{|c|}{ Transcription } \\
\hline Gene Symbol & Fold reg. & Gene Symbol & Fold reg. \\
\hline Pou3f1 & 3,58 & St18 & 2,60 \\
\hline Egr2 & 3,56 & Maf & 2,37 \\
\hline Trps1 & 2,02 & Rcor3 & 2,36 \\
\hline \multicolumn{2}{|l|}{ Cytoskeleton } & Zfp192 & 2,17 \\
\hline Gene Symbol & Fold reg. & \multicolumn{2}{|l|}{ Signalling } \\
\hline Acta2 & 4,05 & Gene Symbol & Fold reg. \\
\hline Ncald & 2,36 & Ptprz1 & 2,03 \\
\hline Myl6 & 2,27 & \multicolumn{2}{|l|}{ Metabolism } \\
\hline \multicolumn{2}{|l|}{ Signalling } & Gene Symbol & Fold reg. \\
\hline Gene Symbol & Fold reg. & Ltb4dh & 4,30 \\
\hline Ctsc & 3,22 & \multicolumn{2}{|c|}{ Behaviour, learning and memory } \\
\hline Adcy1 & 2,03 & Gene Symbol & Fold reg. \\
\hline Phb2 & 2,02 & Prkar2b & 3,19 \\
\hline \multicolumn{2}{|c|}{ Behaviour, learning and memory } & Ptgds & 2,22 \\
\hline Gene Symbol & Fold reg. & Hpca & 2,02 \\
\hline $\operatorname{ttr}$ & 6,63 & & \\
\hline
\end{tabular}

Tab. 5 Genes that are differentially regulated in NEX null mutants versus wildtype animals after 7 days of exposure to the enriched environment

\begin{tabular}{|c|c|c|c|}
\hline \multirow{2}{*}{\multicolumn{2}{|c|}{$\begin{array}{l}\text { Genes downregulated in } \mathrm{KO} \\
\text { Transcription }\end{array}$}} & \multicolumn{2}{|c|}{ Genes upregulated in $\mathrm{KO}$} \\
\hline & & \multicolumn{2}{|c|}{ Transcription } \\
\hline Gene Symbol & Fold reg. & Gene Symbol & Fold reg. \\
\hline Pou3f1 & 4,83 & Yap1 & 3,15 \\
\hline Npas4 & 3,16 & Egr2 & 2,70 \\
\hline Mrg1 & 2,46 & Etv3 & 2,29 \\
\hline $\mathrm{Ldb} 2$ & 2,27 & & \\
\hline $\mathrm{Nr} 4 \mathrm{a} 2$ & 2,25 & \multicolumn{2}{|l|}{ Signalling } \\
\hline \multicolumn{2}{|l|}{ Cytoskeleton } & Gene Symbol & Fold reg. \\
\hline Gene Symbol & Fold reg. & Cnih3 & 3,07 \\
\hline Epb4.112 & 2,71 & Dusp14 & 2,83 \\
\hline Scin & 2,20 & Plekha2 & 2,62 \\
\hline \multicolumn{2}{|l|}{ Signalling } & Pcsk5 & 2,47 \\
\hline Gene Symbol & Fold reg. & Camk1 & 2,35 \\
\hline Dusp5 & 4,09 & Inpp4b & 2,22 \\
\hline Sostdc1 & 3,09 & Pcsk5 & 2,11 \\
\hline Ctsc & 2,42 & Trib2 & 2,10 \\
\hline Frzb & 2,35 & \multicolumn{2}{|l|}{ Metabolism } \\
\hline Gng4 & 2,20 & Gene Symbol & Fold reg. \\
\hline \multicolumn{2}{|l|}{ Metabolism } & Fmo1 & 2,08 \\
\hline Gene Symbol & Fold reg. & \multicolumn{2}{|c|}{ Behaviour, learning and memory } \\
\hline Arg2 & 4,01 & Gene Symbol & Fold reg. \\
\hline \multirow[t]{2}{*}{ Cyp4x1 } & 2,08 & Prkar2b & 3,98 \\
\hline & & jun & 2,60 \\
\hline
\end{tabular}




\begin{tabular}{|l|c|l|c|}
\hline \multicolumn{4}{|l|}{ Tab. 4 (continued) } \\
\hline \multicolumn{2}{|l|}{ Genes downregulated in KO } & \multicolumn{2}{l|}{ Genes upregulated in KO } \\
\hline \multicolumn{2}{|l|}{ Cell Growth and Cell Cycle } & \multicolumn{2}{|l|}{ Behaviour, learning and memory } \\
\hline Gene Symbol & Fold reg. & Ier5 & 2,22 \\
\hline Crim1 & 3,02 & ttr & 3,89 \\
\hline Ccnd1 & 2,45 & Cell Growth and Cell Cycle \\
\hline Mas1 & 2,23 & Gene Symbol & Fold reg. \\
\hline \multicolumn{1}{|l|}{ Ion channels and synapse } & Inhba & 3,52 \\
\hline Gene Symbol & Fold reg. & Cdkn1a & 2,65 \\
\hline Cacng5 & 2,56 & Nedd9 & 2,47 \\
\hline Cadps2 & 2,52 & Reln & 2,27 \\
\hline
\end{tabular}

A subset of candidate genes that displayed differential expression in null mutants compared to wildtype animals was chosen to verify microarray data by QRT-PCR (Fig. 6). Calbindin was found to be upregulated in the null mutants in regular cage housing, but not in the enriched environment housing (Fig. 6A). The expression of transthyretin (ttr) was highly upregulated in wildtype animals following 2 days of environmental enrichment; in contrast, mutants showed a much weaker increase in ttr mRNA level (Fig. 6C). Finally, a subset of genes, including cdkn1a (cyclin-dependent kinase inhibitor 1a) and nedd9 (neural precursor cell expressed, developmentally down-regulated gene 9), whose expression showed no changes in wildtype animals, was upregulated in the mutants after 7 days of exposure to complex cages (Fig. 6 E,G). Changes in candidate gene expression levels were confirmed by QRT-PCR (Fig. 6 $\mathrm{B}, \mathrm{D}, \mathrm{F}, \mathrm{H})$. Where differential expression was observed at only one time point, QRT-PCR was performed only for this time point ( 0 for calbindin; 7 days of enriched environment for cdkn1a and nedd9). 
A

calb1

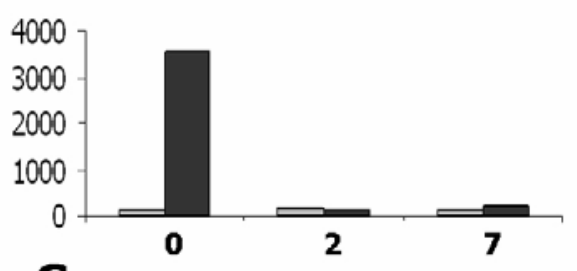

C

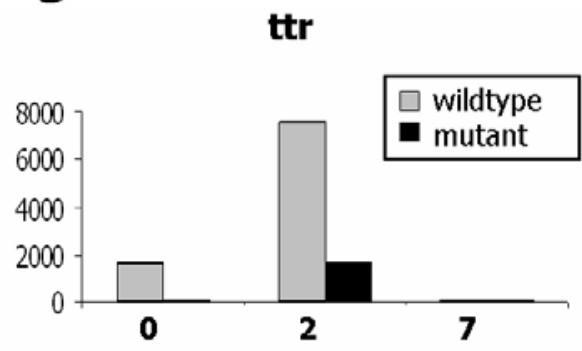

E

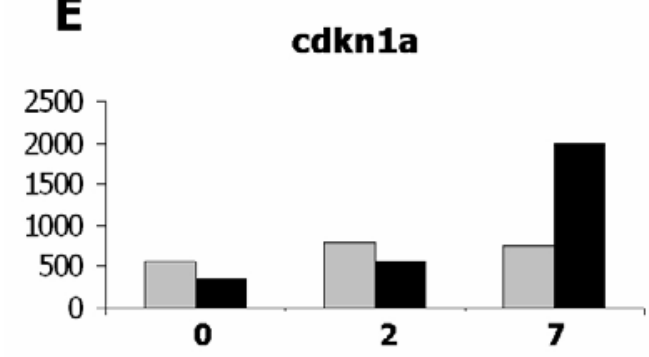

\section{G} nedd9

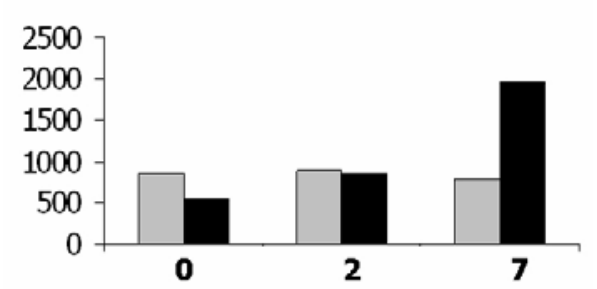

B

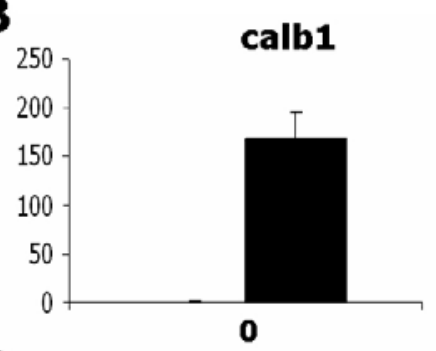

D

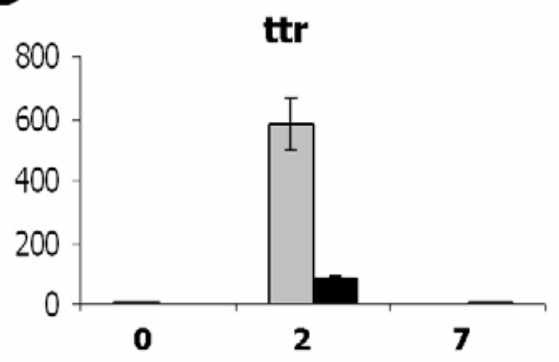

$\mathbf{F}$

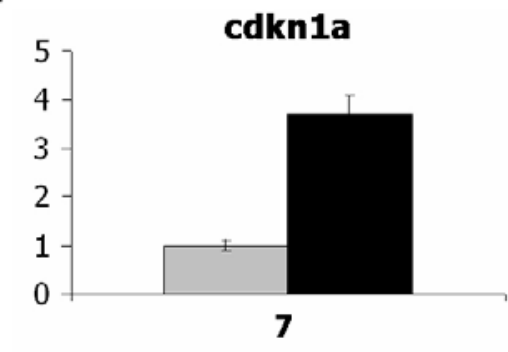

H

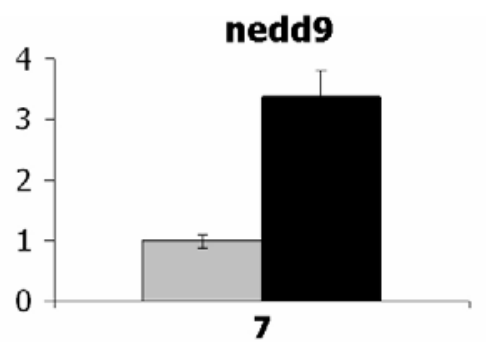

Fig. 6 Differential gene expression in NEX null mutants and wildtype animals upon environmental enrichment: comparison of microarray and QRT-PCR data

Differential gene expression, revealed by microarray analysis (left) was confirmed by QRT-PCR (right). Diagrams represent expression levels of calbindin (calb1; A, B), transthyretin (ttr; C, D), cdkn1a (cyclin-dependent kinase inhibitor 1a; E, F) and nedd9 (neural precursor cell expressed, developmentally down-regulated gene 9; G, H).

0,2 and 7 indicate the regular cage housing (0) and 2 and 7 days housing in enriched cages, respectively. Error bars represent SEM. 


\subsection{Behavioural analysis of NEX null mutants}

As mentioned in the previous chapter, microarray data indicated higher metabolic activity in NEX null mutants compared to wildtype mice. Such an increase in metabolism is usually associated with environmental enrichment and higher physical activity; however, in the NEX null mutants it was observed already in the regular cage setup. Additionally, NEX mutant mice displayed differential expression of genes implicated in learning and memory, such as fos, penk1, prkar2b, jun and ttr (see Tab. 3-5). In line with previously published observations that NEX is a putative target of transcription factor CREB (Uittenbogaard et al., 2007) and facilitates expression of neuronal plasticity-related genes in vitro (Uittenbogaard and Chiaramello, 2004; Uittenbogaard and Chiaramello, 2005), these data indicate a role of NEX in higher cognitive functions in vivo. Therefore, a detailed behavioural analysis of NEX mutants was performed in this study. NEX null mutants have a normal life span and exhibit no obvious deficits in mating or cage behaviour and thus are suitable for behavioural and memory testing. Two cohorts of experimental animals were used for a behavioural analysis:

- First cohort: 10 wildtype males and 14 null mutant males

- Second cohort: 5 wildtype males, 4 null mutant males, 4 wildtype females and 6 null mutant females.

Prior to breeding of behavioural cohorts, the NEX null mutant mouse line had been backcrossed more than 14 times to $\mathrm{C} 57 \mathrm{~B} 16 \mathrm{~N}$ background. The experimental animals for behavioural studies were generated by either crossing heterozygous animals to each other or crossing the same heterozygous male to a wildtype and a homozygous null mutant female. In the former case, wildtype and null mutant animals of the same litter were taken for behavioural tests. In the latter case, the offspring of both mothers was housed together until weaning. Therefore, pups of both genotypes, although not belonging to the same litter, had the same father and experienced maternal care of both wildtype and null mutant mothers. Both cohorts were housed under standard conditions in the animal facility before training, but mice of the second cohort were subjected to 7 days of "handling" immediately prior to behavioural testing, i.e. each mouse was handled by the experimentator for 2 minutes every day. This pre-handling was performed in order to minimize the effect of anxiety, caused by handling per se, during the behavioural testing. 


\subsubsection{Evaluation of general physical fitness of NEX null mutants}

General features, such as weight, pain sensitivity, vision acuteness and motor performance in the accelerating rotarod task, were measured in order to make sure that mutant mice are similar in these parameters to the wildtype control animals and therefore appropriate for behavioural assays. Because of the natural differences between genders, weight comparison of the second cohort was made for both genders separately. No difference between NEX null mutants and wildtype animals was observed (Fig. 7A). The acuteness of vision was measured in a visual cliff test (Crawley, 2000). Each mouse was given 10 consecutive trials on the same day. Mice of both genotypes showed a tendency of approximately $70 \%$ to step down to the virtually safe area (Fig. 7B). The hot plate test of general pain sensitivity was performed for both cohorts, and no difference in latency to lick the hind paw was observed between genotypes (Fig. 7C).

General motor performance was assessed in the second cohort by means of an accelerating rotarod. Each mouse was placed onto the rotating rod, whose speed then accelerated from 3 to $30 \mathrm{rpm}$ over the course of 5 minutes. The latency to fall off and the maximal tolerated rotation speed were recorded. NEX mutant mice exhibited higher latency to fall off (Fig. 7D); however, the difference was statistically significant only on the fourth day of training $(p=0,02)$.
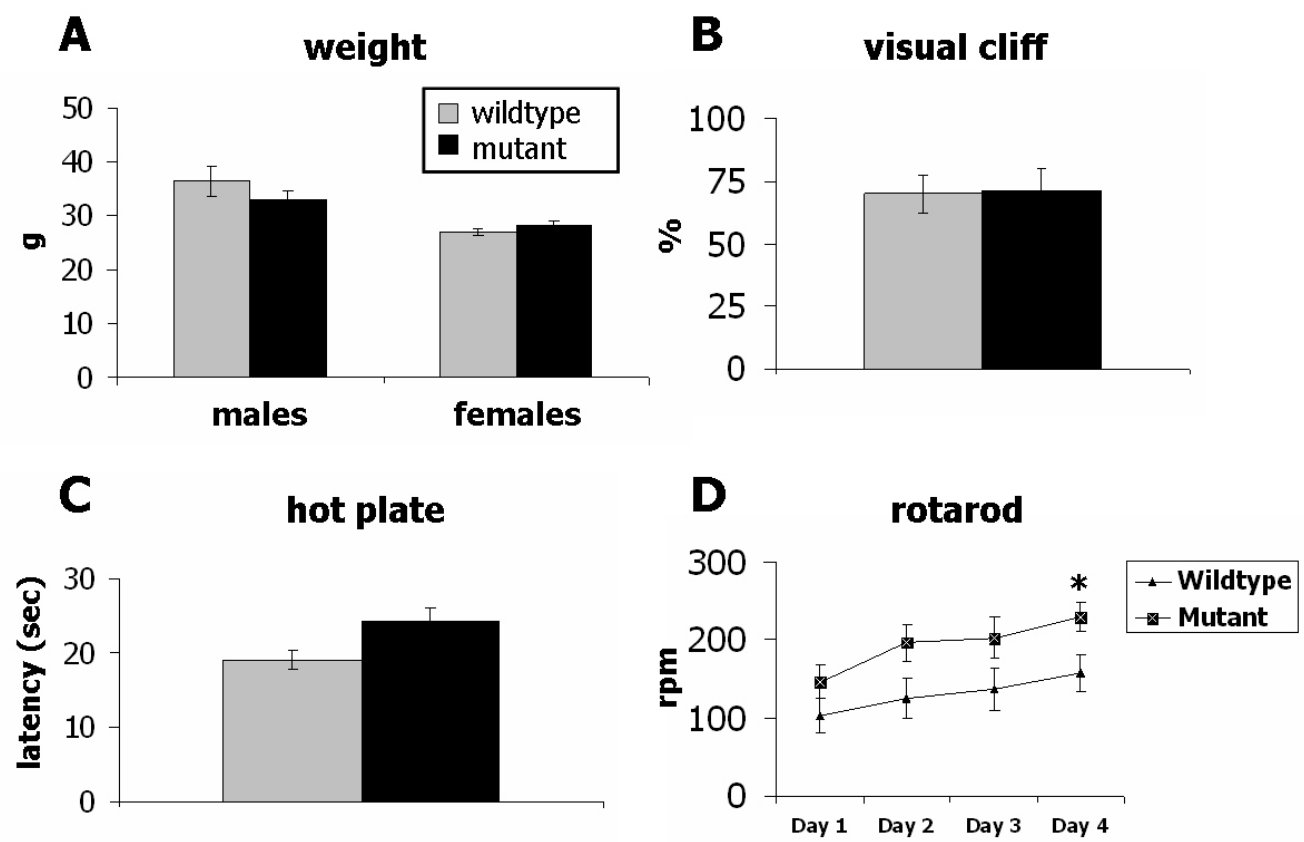

Fig. 7 General physical fitness is unaltered in NEX null mutants

(A) Mutants of both genders do not differ in weight from wildtype animals

(B) Relative tendency to step down to a "safe" area in the vision acuteness test is the same in NEX null mutant and wildtype mice

(C) NEX null mutants have a slightly higher latency to lick the hind paw in the hot plate pain sensitivity test (first cohort, $\mathrm{p}=0,057)$. Similar results were obtained for the second cohort.

(D) NEX null mutants show superior performance in accelerating rotarod motor task to that of wildtype animals (second cohort; ${ }^{*} \mathrm{p}<0,05$ ). Error bars represent SEM. 


\subsubsection{NEX null mutants display hyperactivity in the open field}

Each mouse was allowed to explore an open field box $(45 \times 45 \mathrm{~cm})$ for a period of 10 minutes, after which it was returned to the home cage. The statistical analysis of various activity parameters showed that although NEX mutants (similar to wildtype mice) tend to stay at the periphery of the box (Fig. 8A), they spent more time moving than their wildtype littermates (Fig. 8B-D). This increased locomotory activity was detected in both cohorts, and in the second cohort in both genders (Fig. 8C,D).

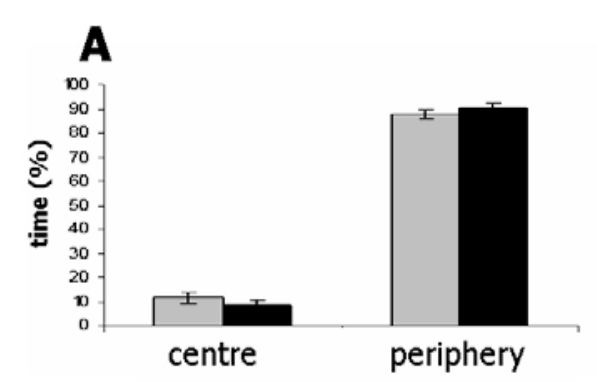

C

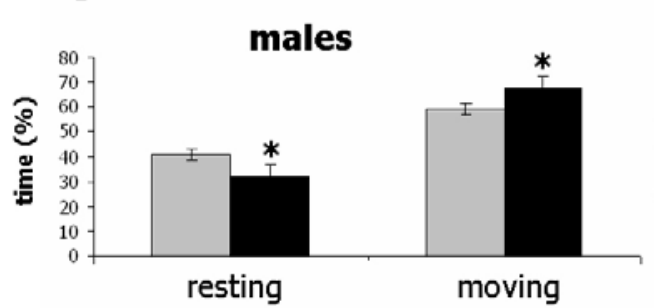

$\mathbf{E}$

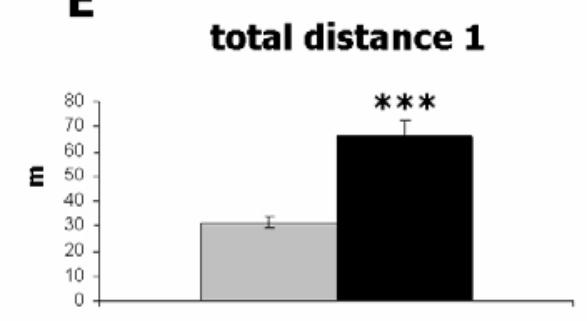

\section{G}

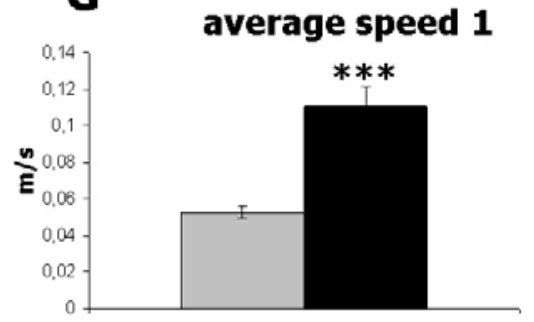

B

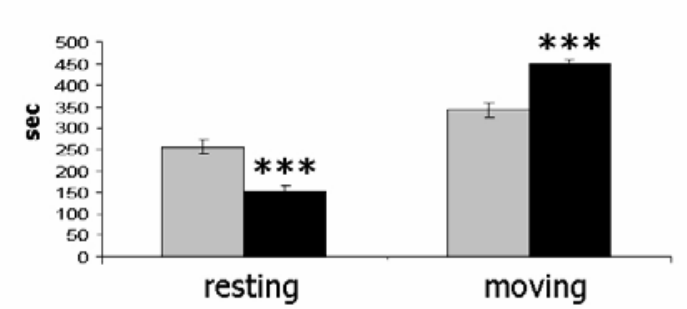

D
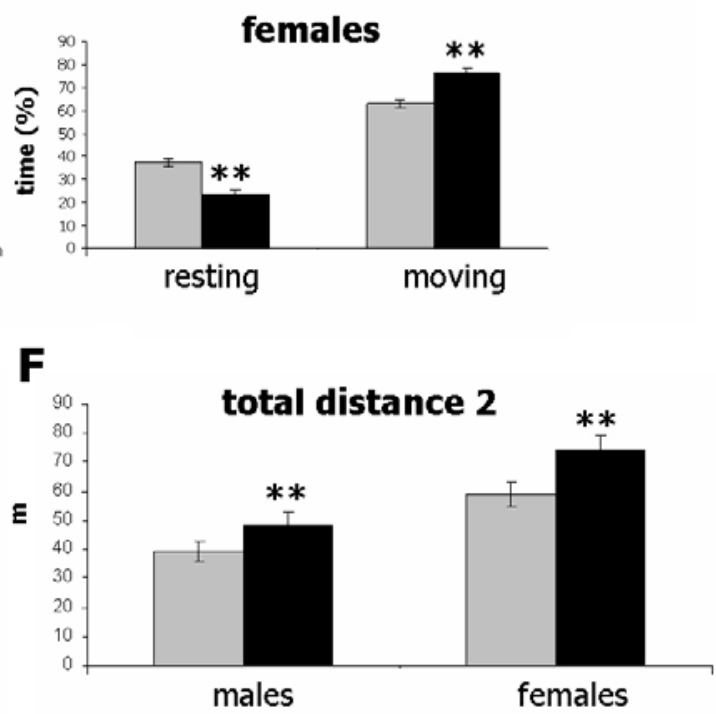

H

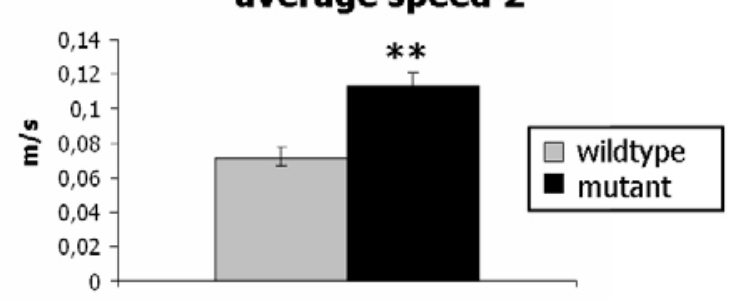

Fig. 8 NEX mutants exhibit hyperactivity and higher velocity in the open field

(A) Both mutants and wildtype controls spend relatively more time at the periphery than in the centre of the open field box. Mutant mice spend relatively more time moving than wildtype animals (B-D). Total distance travelled in the open field box $(\mathbf{E}, \mathbf{F})$ and average velocity $(\mathbf{G}, \mathbf{H})$ was higher for mutants from both cohorts.

(* $\mathrm{p}<0,05 ; * * \mathrm{p}<0,01 ; * * * \mathrm{p}<0,001 ;$ error bars represent SEM)

Hyperactivity was also reflected by the overall distance travelled in the open field, which was significantly higher for mutants of both cohorts compared to their wildtype littermates (Fig. 
8E,F). Moreover, the average velocity of NEX mutants was also significantly higher than that of wildtype control animals (Fig. 8G,H).

\subsubsection{Assessment of general anxiety and fear in NEX null mutant mice}

To assess general anxiety and fear levels in NEX mutants, light-dark preference and plus maze tests were used. Scoring was performed manually in both tests. Typically, general anxiety and fear in mice are represented by the tendency to keep to dark or closed places (similar to staying at the periphery of the open field box). NEX null mutants and wildtype animals showed similar levels of basic anxiety and fear in the open field (Fig. 8A), light-dark box (Fig. 9A) and plus maze (Fig. 9B). However, NEX mutants also exhibited hyperactivity in the light-dark box and plus maze, as demonstrated by the increased number of crossings between compartments in both tests (Fig. 9C,D).
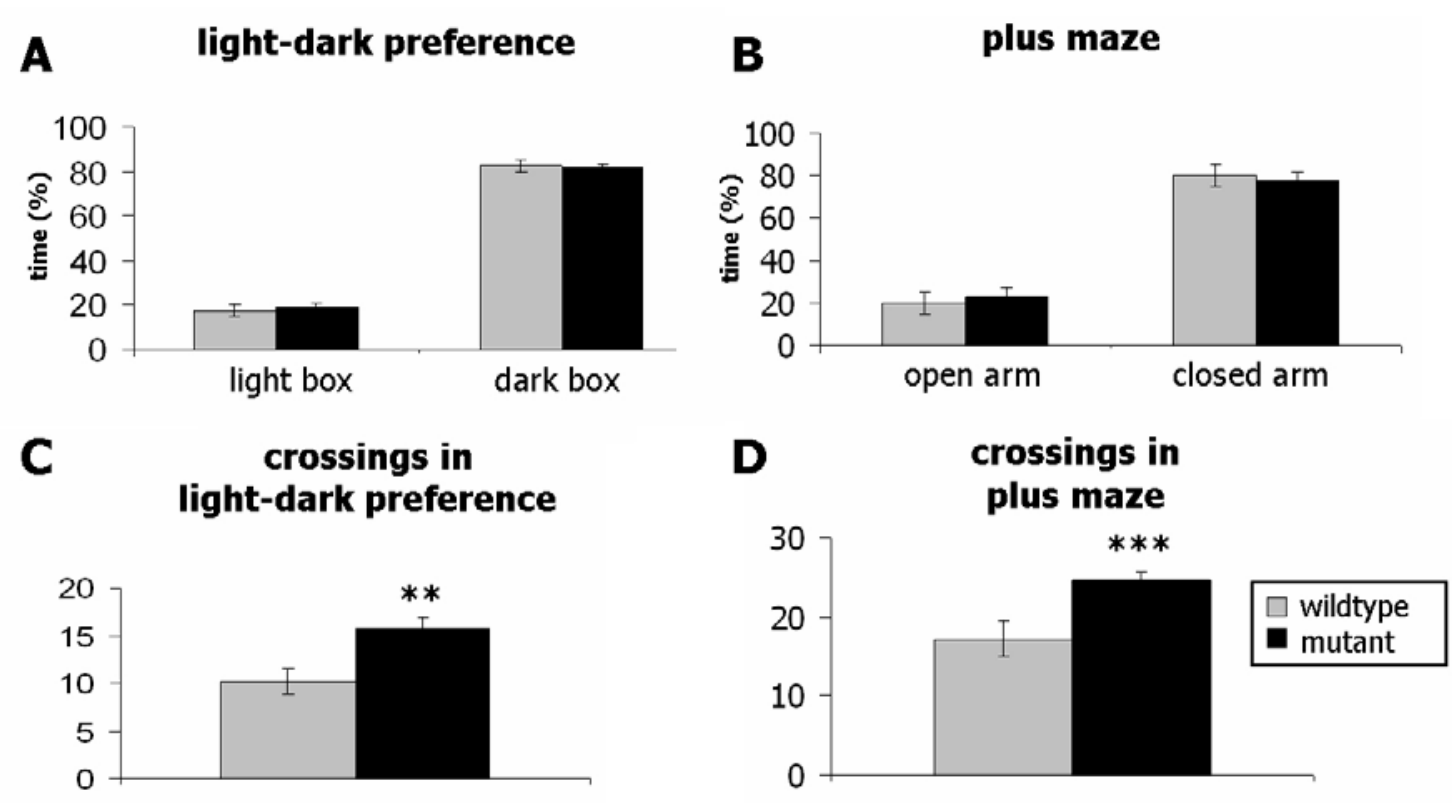

Fig. 9 NEX mutants show normal level of general anxiety in light-dark preference and plus maze

Relative time spent in the light box and dark box in the light-dark preference test (A) is similar in mutants and wildtype animals. Mutants did not differ from wildtype controls in relative time spent in the open and closed arms of the plus maze (B). Mutants show elevated number of crossings in both light-dark preference (C) and plus maze (D) tests.

(** $\mathrm{p}<0,01 ; * * * \mathrm{p}<0,001$; error bars represent SEM) 


\subsubsection{Spatial learning in the Morris water maze}

Sustained NEX mRNA expression in the adult hippocampus, entorhinal cortex and subiculum suggests that NEX might be involved in hippocampus-dependent cognitive functions, such as spatial and contextual memory formation. To assess spatial memory formation in NEX null mutants, animals of both behavioural cohorts were trained in the Morris water maze spatial navigation task.

The first cohort was subjected to 2 days of visible platform training followed by 7 days of hidden platform training, after which a probe trial was performed. In the probe trial the relative time spent in each quadrant of the water maze was measured to assess the ability of mice to remember the platform location. Mutant mice exhibited significantly higher average swimming speed when compared to wildtype animals (Fig. 10A). They also showed slower learning in the visible platform task (Fig. 10B). In the hidden platform task, NEX mutants covered a longer distance to trace the platform; however, the learning curve exhibited a similar slope to that of control animals (Fig. 10C). While control animals successfully mastered the spatial learning task, as shown by their target quadrant preference in the probe trial, NEX null mutants showed lower preference to the target quadrant (Fig. 10D).
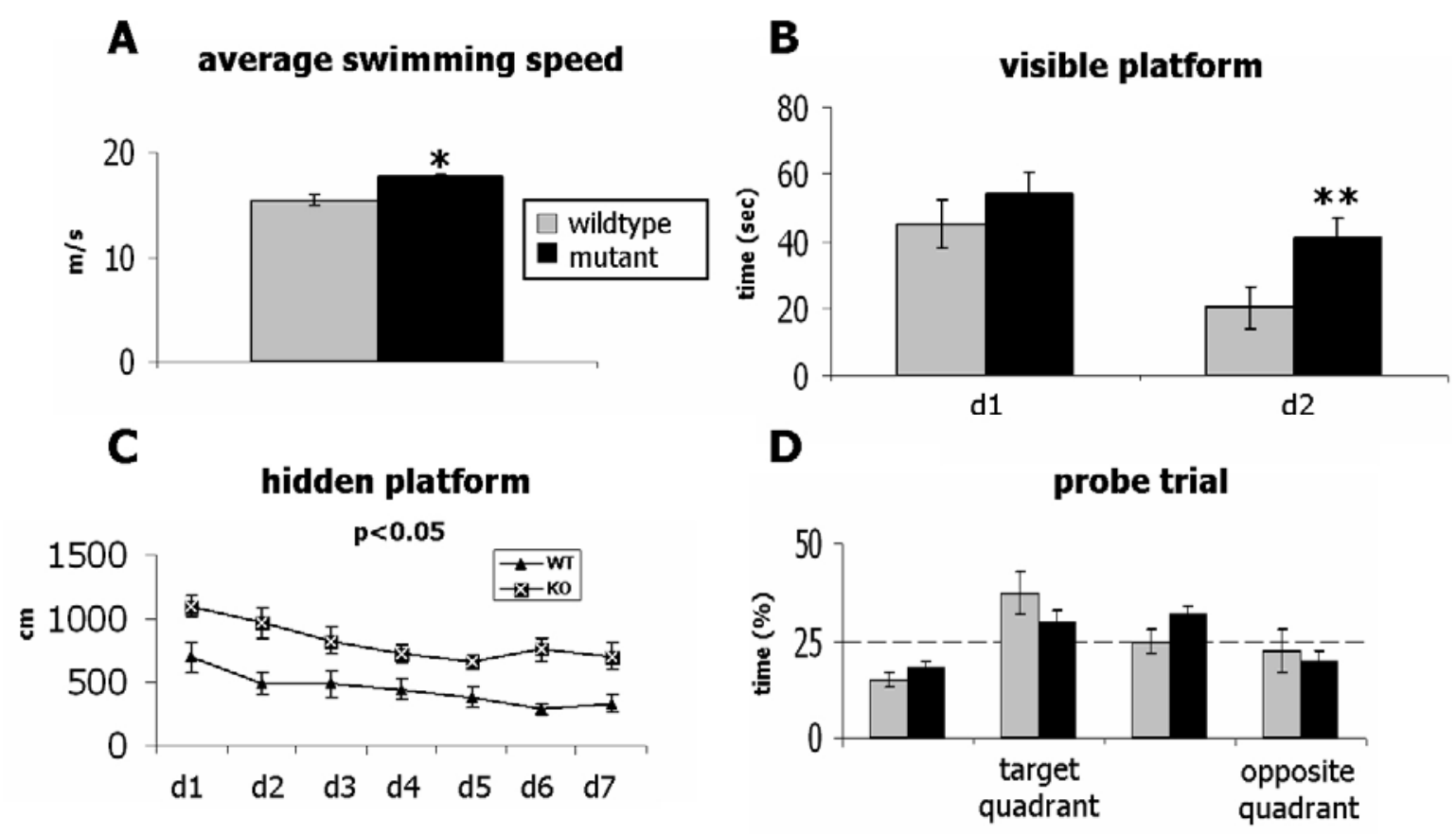

Fig. 10 NEX mutants of the first behavioural cohort show spatial learning deficits in the Morris water maze NEX null mutants exhibit higher average swimming speed in the hidden platform task (A). The latency to reach the platform in the visible platform task is higher in mutants on the second training day (B). Mutants travel longer distance in the hidden platform task (C) and perform worse than wildtype animals in the probe trial on day 8 of the hidden platform training (D).

(* $\mathrm{p}<0,05 ; * * \mathrm{p}<0,01$; error bars represent SEM) 
In order to reveal a possible link between training order and spatial memory formation, the water maze learning setup was slightly changed for the second experimental cohort. The experimental animals were first allowed to master the hidden platform task for 14 consecutive days and then subjected to the visible platform task for 3 days. Probe trials were performed on day 7 and 14 of the hidden platform training. Statistical analysis showed no difference in distribution between genders within the same genotype, therefore, the data obtained for males and females was pooled. The hidden platform learning was similar to that of the first cohort: NEX null mutants, although able to learn the task, performed significantly worse that their wildtype littermates (Fig. 11A). However, no difference between genotypes was detected in the subsequent visible platform training (Fig. 11B). For control mice, the preference for the target quadrant, already pronounced at day 7 (Fig. 11C), further increased towards the end of training (Fig. 11D). In contrast, NEX null mutants showed only slight preference to the target quadrant, which did not increase with prolonged training (Fig. 11C,D).
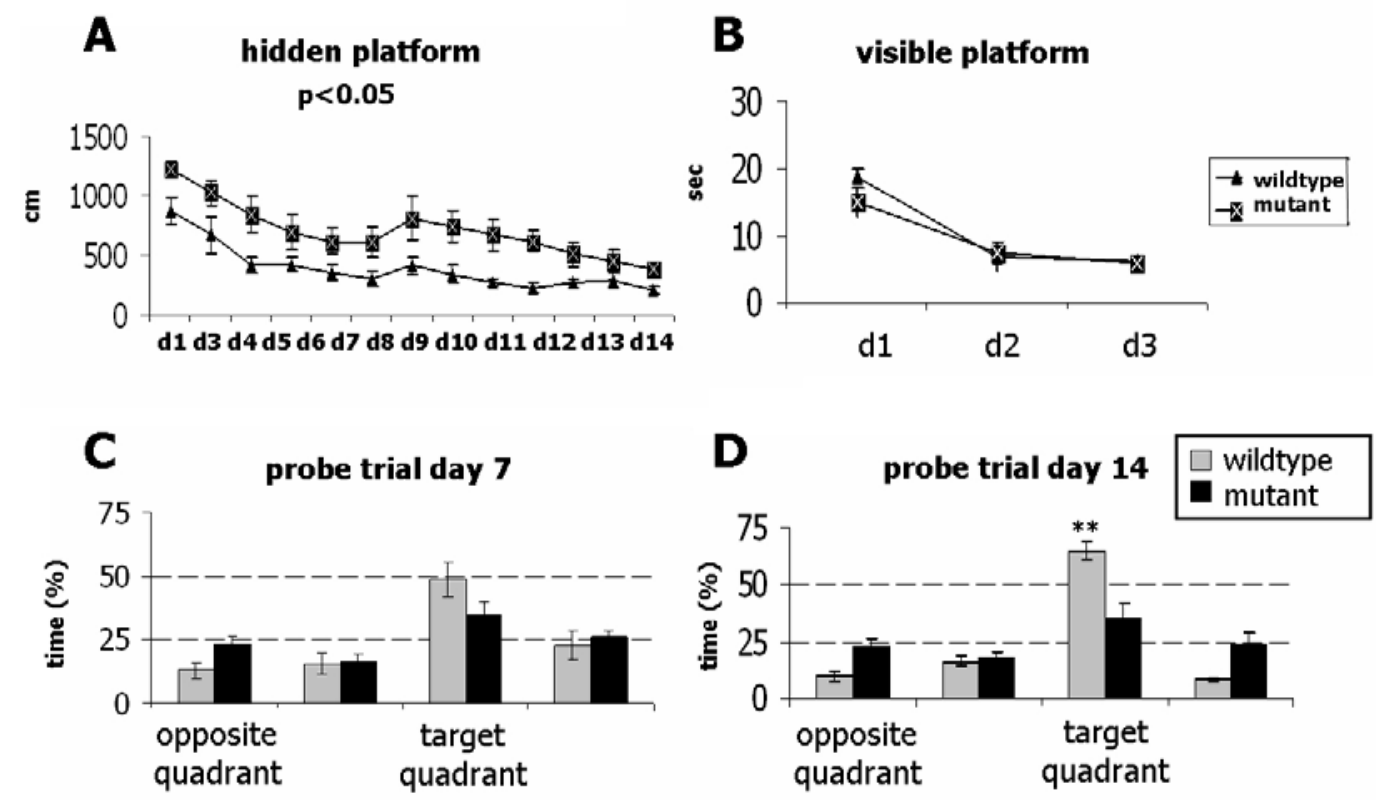

Fig. 11 NEX mutants of the second behavioural cohort show spatial learning deficits in the Morris water maze

NEX null mutants travel longer distance than wildtype controls in the hidden platform task (A), but show no deficit in the visible platform task (B). Mutants perform worse than wildtype animals in the probe trial on day 7 (C) and day 14 (D) of the hidden platform training. $(* * p<0,01$; error bars represent SEM)

Comparison of different training paradigms revealed the paradigm effect on the performance in the water maze tasks. Two learning processes typically involved in the training, i.e. spatial learning per se and association between the platform and escape, can be thus uncoupled. Placing visible platform training at the end rather than at the beginning of testing resulted in the decrease of time (Fig. 10B; Fig. 11B) and distance (Fig. 12A,B) required for the mouse to reach the visible platform. Similarly, when hidden platform training is not preceded by 
visible platform training, the platform is not viewed as means of escape, which results in poorer performance during the first days of hidden platform training. Thus, wildtype mice of the first cohort (for whom visible platform training preceded hidden platform task) showed better performance on the first day of hidden platform task than wildtype mice of the second cohort (Fig. 12C,D). The trial-by-trial analysis of the performance of first cohort on the first day of the hidden platform task also revealed the failure of NEX null mutants to improve over 4 trials (Fig. 12C).
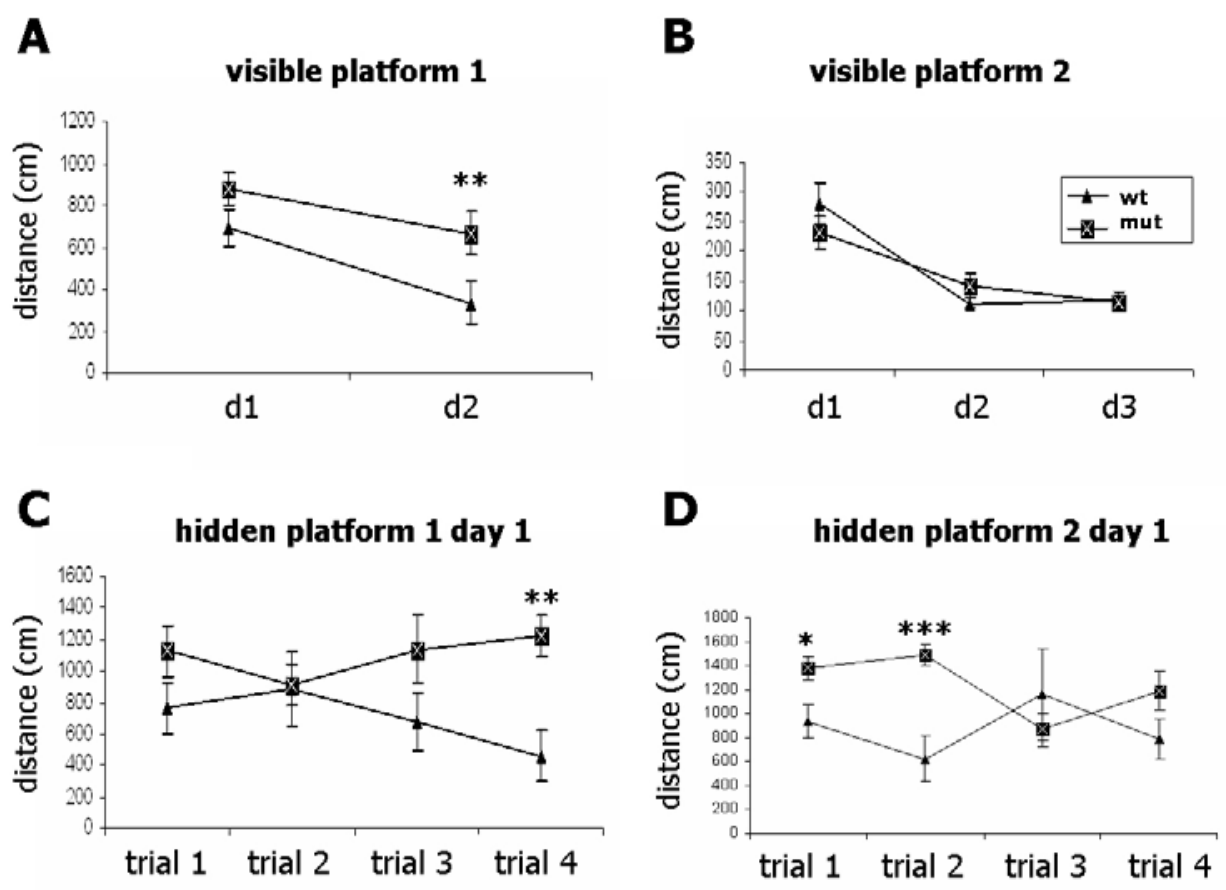

Fig. 12 The effect of different training paradigms on performance in the Morris water maze Mutants of the first cohort (A) are impaired in finding the platform on the second day of the visible platform task, whereas mutants of the second cohort have mastered the visible platform task with the same efficiency as control animals (B). Mutants of both cohorts show impaired performance on the first training day of hidden platform task (C,D). (* $\mathrm{p}<0,05 ; * * \mathrm{p}<0,01$; error bars represent SEM)

\subsubsection{Recent and remote memory formation and extinction}

Fear conditioning was used to evaluate cued and contextual fear memory formation and stability in the NEX null mutants compared to wildtype controls. Both cohorts were subjected to conditioning to cue (tone) and context (conditioning cage). Scoring of freezing response during conditioning and subsequent extinction training was done manually online.

The first cohort was tested for fear memory of both cue and context the day after conditioning took place (recent memory), after which contextual memory extinction was performed for 8 consecutive days. The cue (tone) memory testing revealed no difference in the strength of fear memory between NEX null mutant and wildtype mice (Fig. 13A). However, NEX null mutants showed a significant decrease in contextual fear memory when compared to 
control animals (Fig. 13B). Fear memory extinction produced a significant decrease in freezing in wildtype animals, but no such decrease was observed for NEX null mutants (Fig. 13C,D).
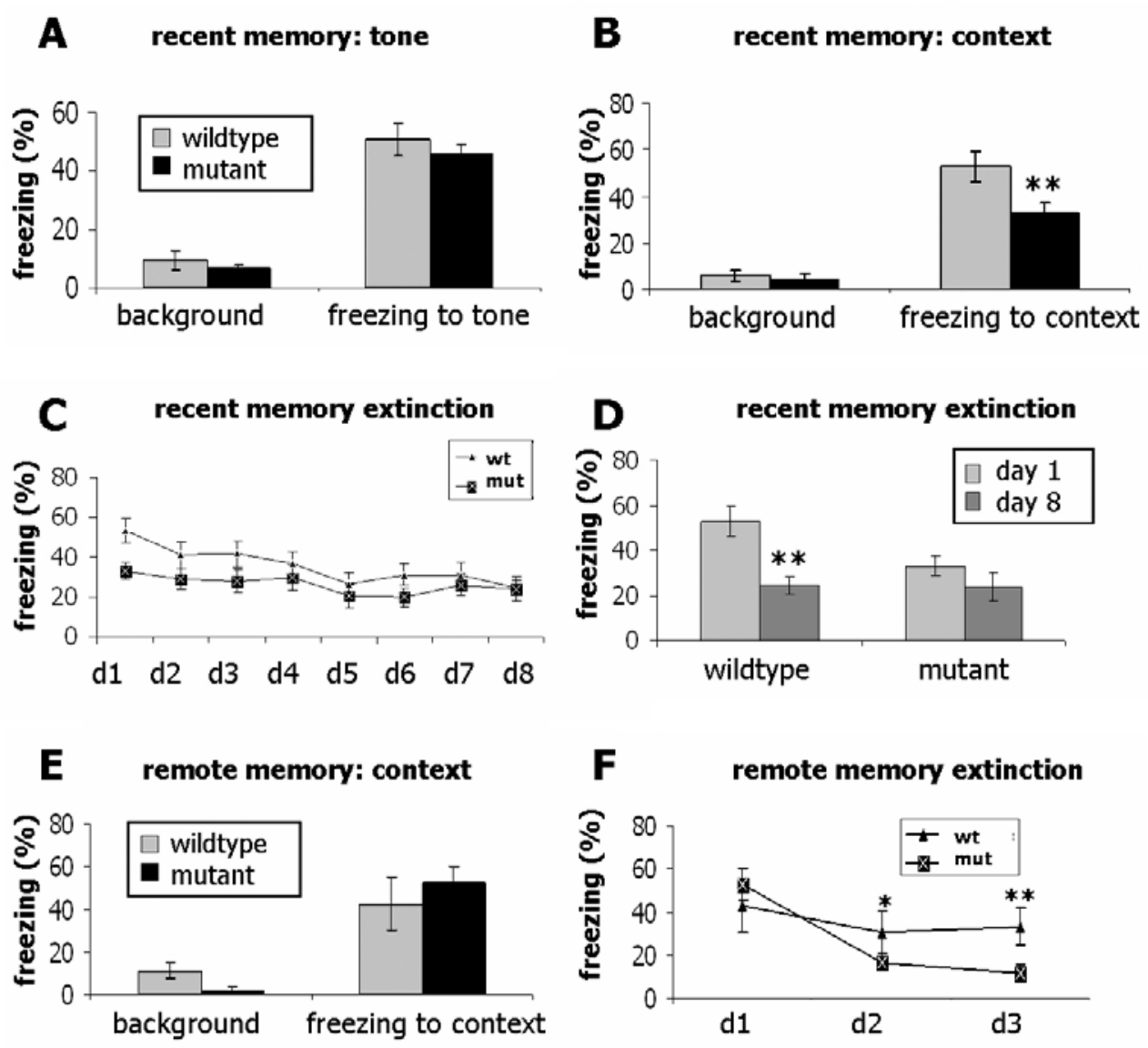

Fig. 13 Recent and remote fear memory extinction in NEX mutants

Mutants of the first cohort show a normal level of freezing response to the conditioned stimulus (tone) (A), but a reduced freezing response to conditioned context (conditioning box) (B) one day after training (recent memory). Wildtype animals demonstrate extinction of recent fear memory over 8 days, whereas the level of freezing response stays constant in the mutants $(\mathbf{C}, \mathbf{D})$. Mutants of the second cohort show normal freezing response to conditioned context (conditioning box) 36 days after training (remote memory) (E), but demonstrate rapid extinction of remote contextual fear memory $(\mathbf{F})$.

(* $\mathrm{p}<0,05 ; * * \mathrm{p}<0,01$; background shows basal level of freezing in a novel environment; error bars represent SEM)

The second cohort was tested for fear memory of context 36 days after conditioning (remote memory), after which extinction was performed. NEX null mutants showed a freezing level comparable to that of wildtype animals, demonstrating a similar level of remote contextual fear memory in both genotypes (Fig. 13E). However, already on the next day the level of freezing in NEX mutants has significantly decreased, whereas wildtype controls maintained approximately the same level of freezing (Fig. 13F). 


\section{Cre recombinase-mediated overexpression of NEX in neural progenitor cells of the Emx1 lineage}

In order to investigate the function of NEX in murine forebrain development, a gain-offunction approach was used to create a transgenic mouse line allowing for Cre-mediated overexpression of recombinant NEX protein. The Cre-loxP system is widely used in mouse genetics for the conditional inactivation of genes in certain tissue and cell types. The technique is based on the ability of Cre-recombinase to catalyse the excision of DNA fragments in a sequence-specific manner by binding to so-called loxP sites. In this study the Cre-loxP system was chosen as a tool for the inducible overexpression of a transgene in the mouse. To allow for Cre recombinase-dependent expression the ORF of the gene of interest is positioned 3' to a reporter ORF that serves as a stop cassette in the transgenic construct. In the absence of Crerecombinase the reporter, in this case enhanced green fluorescent protein (eGFP), is expressed. In the presence of Cre, the stop cassette is excised, and the gene of interest is expressed (see Fig.14A).

\subsection{Production of a mouse line with inducible overexpression of NEX}

An AU1 tag (aminoacid sequence: DTYRYI; see Shevtsova et al., 2006) was added at the N-terminus of the NEX ORF by PCR. A CMV- $\beta$-actin floxed eGFP construct was originally obtained from Dr. Victor Tarabykin (MPI of Experimental Medicine); and further modified by Maike Gummert (MPI of Experimental Medicine). After cloning of AU1-tagged NEX into the CMV- $\beta$-actin vector (see Fig. 14A) the integrity of the transgene construct (GFP-NEX) was verified by sequencing. A functional analysis was performed in PC12 cells. After transfection with the GFP-NEX construct in the presence or absence of CMV-promoter-driven Crerecombinase immunostaining against eGFP and AU1-tagged recombinant NEX was performed (Fig. 14B). Cells that only received the GFP-NEX construct, but not CMV-Cre, were GFPpositive and AU1-negative (arrow in Fig. 14B), those that received both constructs were AU1positive and showed no GFP staining (arrowhead in Fig. 14B). Cre-mediated expression of AU1tagged NEX was also observed on a western blot (Fig. 14C). 


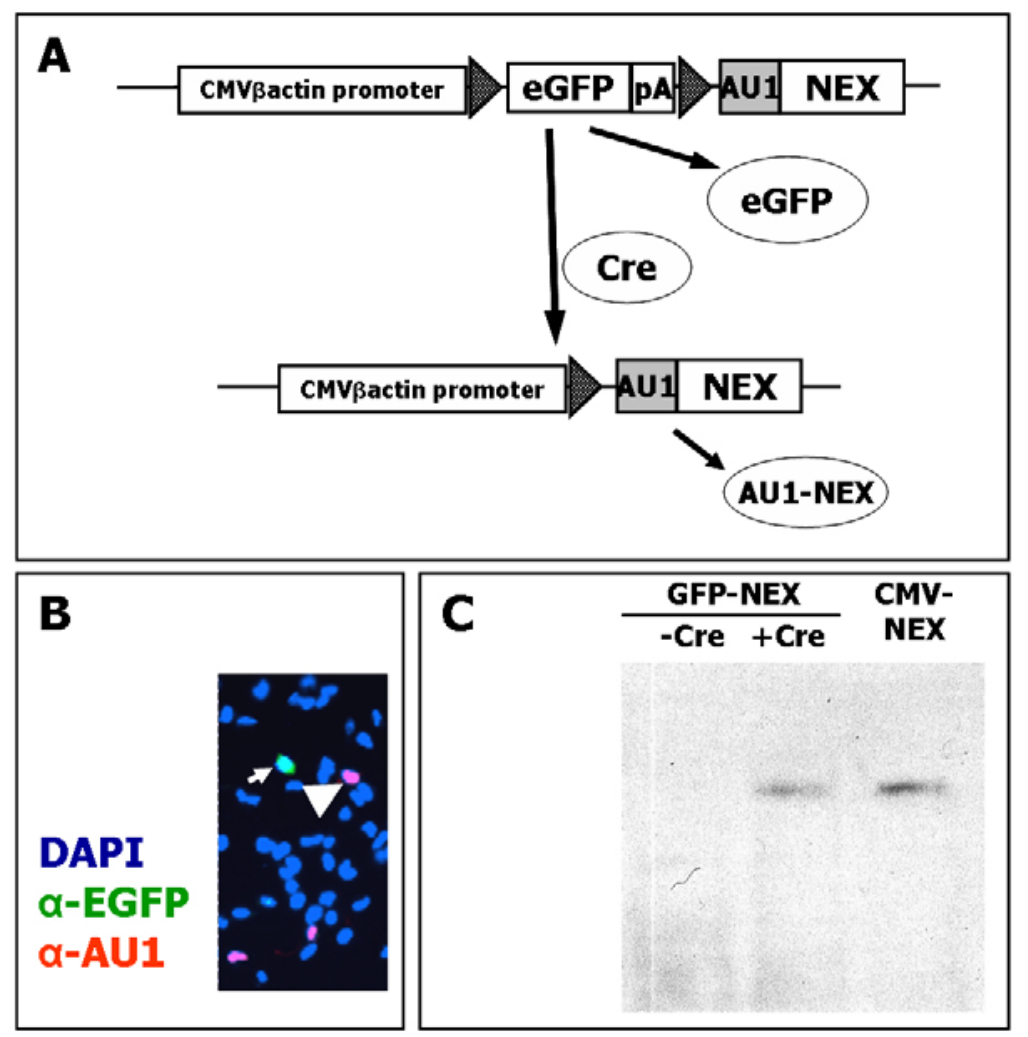

Fig. 14 Functional analysis of the GFP-NEX construct

(A) CMV-ßactin promoter - floxed eGFP (GFP-NEX) construct and its mode of function. Above: the original construct drives expression of eGFP protein. Below: as a result of Cre-recombinase-mediated excision of eGFP ORF, AU1-NEX recombinant protein expression is achieved. Immunocytochemistry on PC12 cells, transfected with the GFP-NEX construct and the CMV-Cre construct demonstrates AU1-tagged protein expression (B). Western blot on the extracts of PC12 cells, transfected with GFP-NEX construct in the presence or absence of Cre-recombinase, demonstrated transgenic NEX protein expression in the former case (C). On the right - extract of PC12 cells, transfected with CMV-NEX construct, used as positive control.

(AU1 - AU1 tag at the N-terminus of NEX ORF; CMV - human cytomegalovirus promoter; Cre - Crerecombinase; DAPI - nuclear stain; eGFP - enhanced green fluorescent protein; pA - poly A site; triangles represent loxP sites)

After functional analysis the GFP-NEX construct was used for the production of transgenic mouse lines. The functional module, shown in Fig. 14A (upper panel), was released from the vector backbone, purified and used for injection into the pronuclei of mouse zygotes. Thirty potential founder mice were screened for green fluorescence and analysed by PCR on genomic tail DNA. Two founders were PCR-positive and displayed ubiquitous fluorescence, but only one of them transmitted the transgene to its offspring. All progeny exhibited uniform green fluorescence when analysed under the inverted microscope equipped with a UV-lamp (Fig. 15A). 


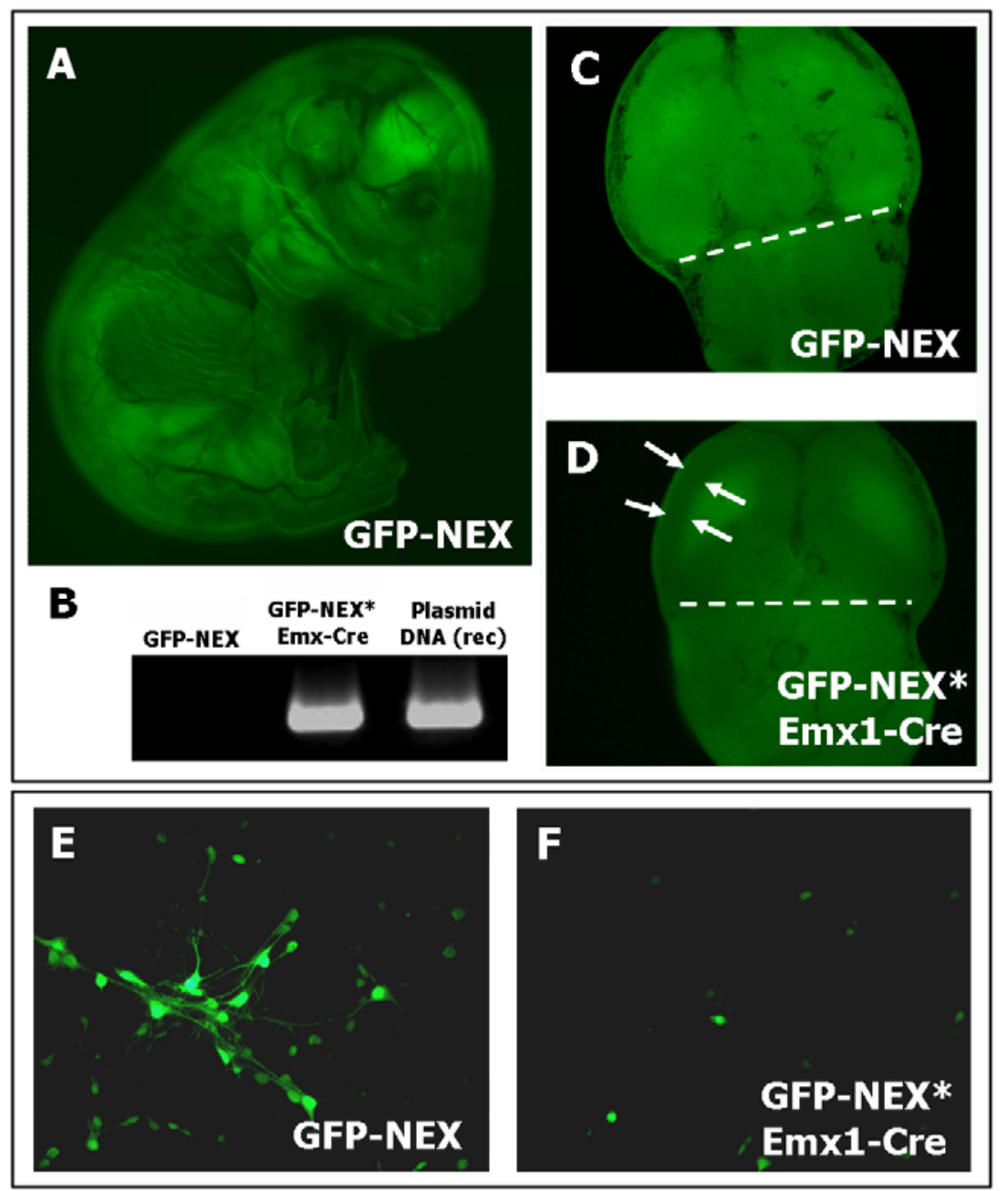

Fig. 15 Cre-mediated recombination in GFP-NEX transgenic mice

GFP-NEX transgenic mice exhibit uniform green fluorescence (A). PCR analysis of GFP-NEX and GFPNEX*Emx1-Cre animals demonstrates recombination in brain genomic DNA of double transgenic animals (B). Recombined GFP-NEX plasmid DNA (right) served as a positive control.

Comparison of green fluorescence in brains of GFP-NEX (C) and GFP-NEX*Emx1-Cre (D) mice at P0 revealed a reduction of fluorescence in the cortical region of GFP-NEX*Emx1-Cre brain (arrows in D). White dashed line separates the cerebral hemispheres from the hindbrain.

The number of fluorescent cells is greatly reduced in cultures of cortical neurons prepared at E16 from GFPNEX*Emx1-Cre mice (F) compared to GFP-NEX single transgenic controls (E) (embryonic neuronal cultures isolated from littermates at E16; same number of cells were present in the visual field).

\subsection{Inducible overexpression of NEX in neural progenitors}

The Emx1-Cre mouse line (Gorski et al., 2002) was used to overexpress AU1-NEX in progenitor cells of the developing mouse telencephalon. PCR on DNA isolated from the brains of single- and double-transgenic animals showed efficient recombination in GFP-NEX*Emx1Cre mice (Fig. 15B). Excision of the eGFP cassette was confirmed by a decrease of fluorescence in the cortical region of GFP-NEX*Emx1-Cre mouse brains as compared to GFP-NEX single transgenic mice (Fig. 15C,D). Primary cultures of cortical neuron were prepared from GFP-NEX and GFP-NEX*Emx1-Cre embryos at E16, and GFP fluorescence was examined. While the vast majority of neurons from GFP-NEX single transgenic animals were GFP-positive (Fig. 15E), in 
GFP-NEX*Emx1-Cre cultures only a few green cells were found, of which none displayed typical neuronal morphology (Fig. 15F).

\subsubsection{Overexpression of NEX in cortical progenitors impairs forebrain development}

GFP-NEX*Emx1-Cre double transgenic animals exhibited an obvious reduction in cerebral hemisphere size in comparison to single transgenic controls (Fig. 16A). The size reduction of cerebral hemispheres was further analysed on whole brain images using the surface area calculation module of ImageJ programme, with the hemisphere surface area of control mice taken as $100 \%$. Statistical analysis of this measurement revealed significant difference in cerebral hemisphere size between control animals and GFP-NEX*Emx1-Cre mutant mice in adulthood (Fig. 16B; $<<0,01$ ) as well as at birth (Fig. 16C; $<<0,01$ ). In contrast, no brain size reduction was observed in double transgenic mice that overexpress NEX in postmitotic pyramidal neurons (GFP-NEX*NEX-Cre; Fig. 16D).

In order to determine the particular defect leading to brain size reduction in GFPNEX*Emx1-Cre transgenic mice, immunohistochemistry for NeuN (a marker of neuronal nuclei) was performed on coronal sections of newborn and adult animals. Although neuronal density in the cortex and hippocampus appeared unchanged, the mutant cortex was thinner at P0 (Fig. 17A,B) and in the adult brain (Fig. 17C,D). NeuN staining of the adult somatosensory cortex revealed disturbed cortical layer formation in double transgenic mice (Fig. 18). Whereas superficial (II-III and IV) cortical layers appeared normal, the size of deeper cortical layers (V and VI) was reduced (Fig. 18), suggestive of impairment that occurred already during early stages of telencephalon development. 

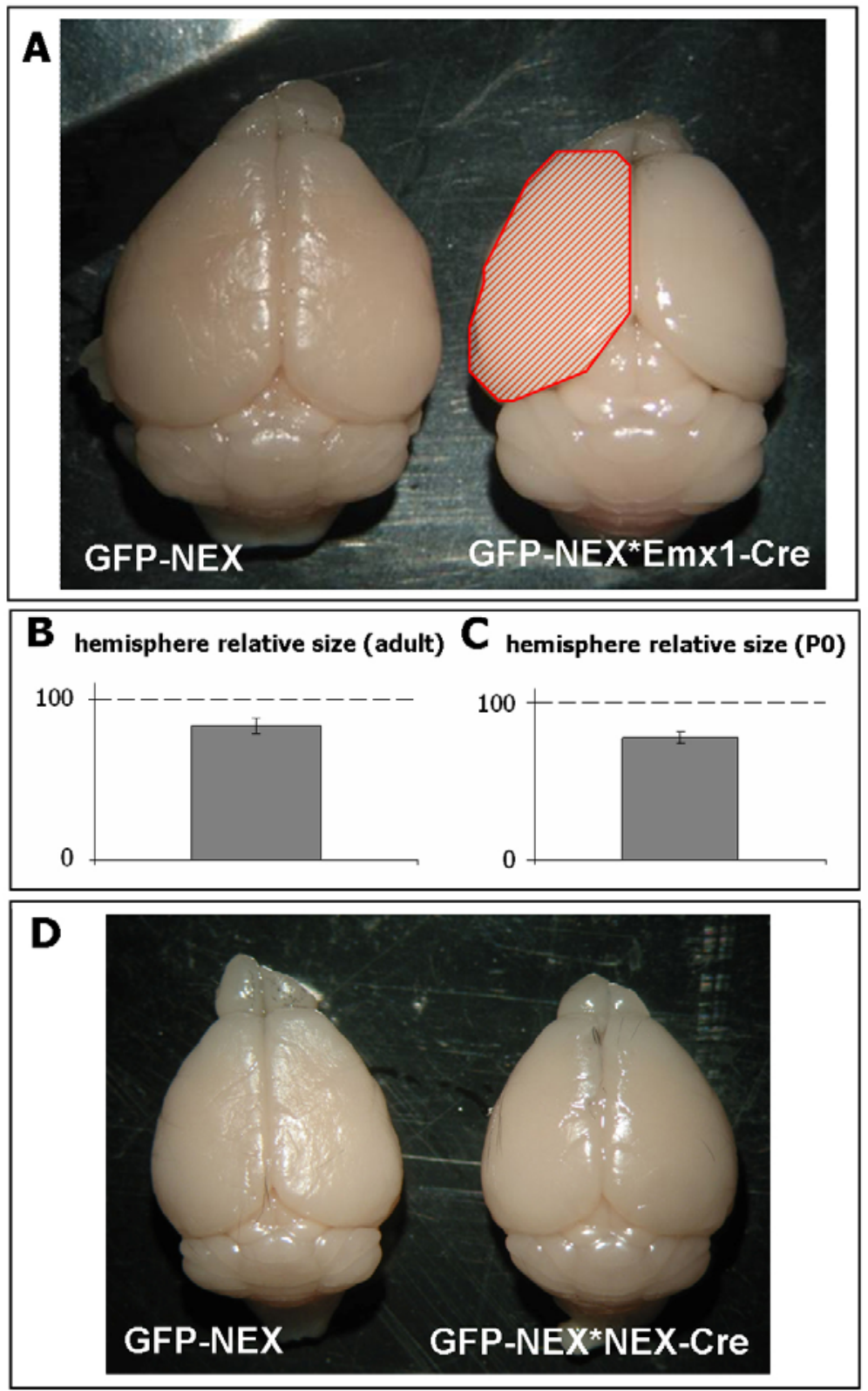

Fig. 16 Reduction of cerebral hemisphere size in GFP-NEX*Emx1-Cre transgenic mice

(A) Dorsal view of the brains of control (left) and GFP-NEX*Emx1-Cre (right) transgenic mice. The red shaded area illustrates the hemisphere surface that was taken for statistical analysis. Hemisphere size of adult mutant mice is significantly smaller than that of control animals; $n=3, p<0,01$ (B). Similar hemisphere size reduction was observed at birth; $n=3, p<0,01(\mathbf{C})$. In contrast, no size difference was observed between control (left) and GFP-NEX*NEXCre (right) brains (D). 

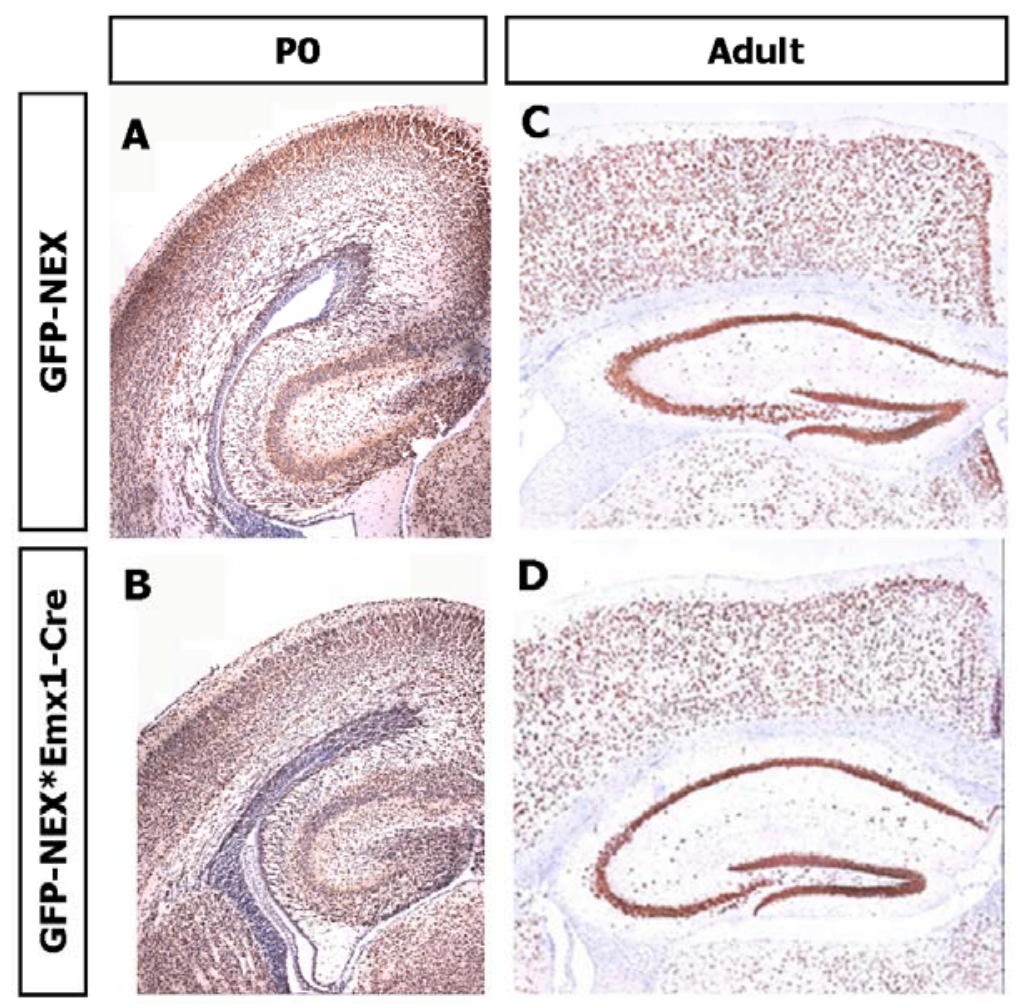

Fig. 17 Cortical size reduction in GFP-NEX*Emx1-Cre transgenic mice

(A, B) $\alpha$-NeuN staining shows cortical size reduction in the GFP-NEX*Emx1-Cre double transgenic mice at P0. Similar reduction was observed in adult animals (C, D) (counterstained with Nissl).

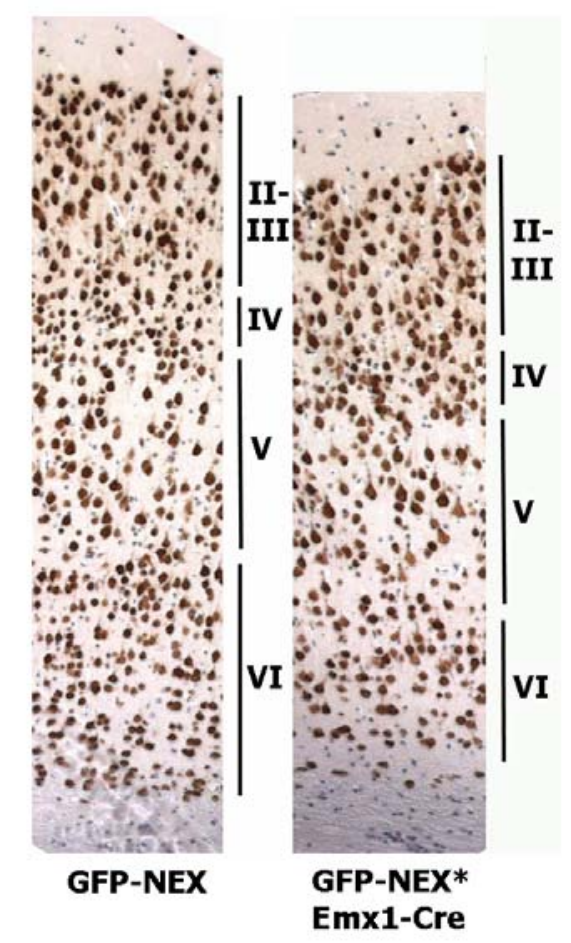

Fig. 18 Abnormal cortical layer formation in GFP-NEX*Emx1-Cre transgenic mice $\alpha$-NeuN immunostaining of control (left) and GFP-NEX*Emx1-Cre (right) adult brain sections revealed cortical lamination defects in the mutant. Roman numbers II-VI represent corresponding cortical layers. 


\subsubsection{Early stages of cortical plate formation in mutant embryos}

Since deeper cortical layers were primarily affected in GFP-NEX*Emx1-Cre double transgenic animals, a more detailed analysis was performed at E12,5 and E13,5. TUNEL staining for apoptotic cells revealed massive apoptosis in the ventricular zone of GFP-NEX*Emx1-Cre mutant mice at E12,5, which was not detected in littermate controls (Fig. 18A,B).Likewise, TUNEL staining performed on saggital sections at E13,5 also demonstrated an increase in the number of apoptotic cells in double transgenic animals (Fig. 19C,D). $\beta$-Tubulin III (Tuj1) immunohistochemistry revealed Tuj1-labelled cells in the ventricular zone of GFP-NEX*Emx1Cre mutant E13,5 embryos, which were not detected in littermate controls (Fig. 19E,F). An ectopic $\beta$-Tubulin III expression could be caused by various deficits in the neocortex development. First, a premature cell cycle exit of the progenitors could trigger differentiation already in the ventricular zone. Second, a similar effect could be achieved as a result of migration defects of the progenitor cells (Berger et al., 2007). Further detailed investigation of early telencephalon development in GFP-NEX*Emx1-Cre transgenic mice will include BrdU pulse-chase experiments and analysis of expression of cell cycle-related markers and adhesion molecules (such as N-cadherin), and therefore help to reveal whether the developmental deficits are caused by the abnormal cell cycle control or migration defects or both.

Although Emx1-Cre-mediated overexpression of NEX provides important information on the role of NEX in cell cycle and migration control, the ectopic expression of a differentiation gene in mitotically active progenitors is to a certain extent artificial and does not reveal the natural function of NEX in telencephalon development. Ongoing investigation of NEX overexpression in postmitotic neurons in GFP-NEX*NEX-Cre double transgenic animals will therefore continue to decipher the in vivo function of NEX during pyramidal cell differentiation. 

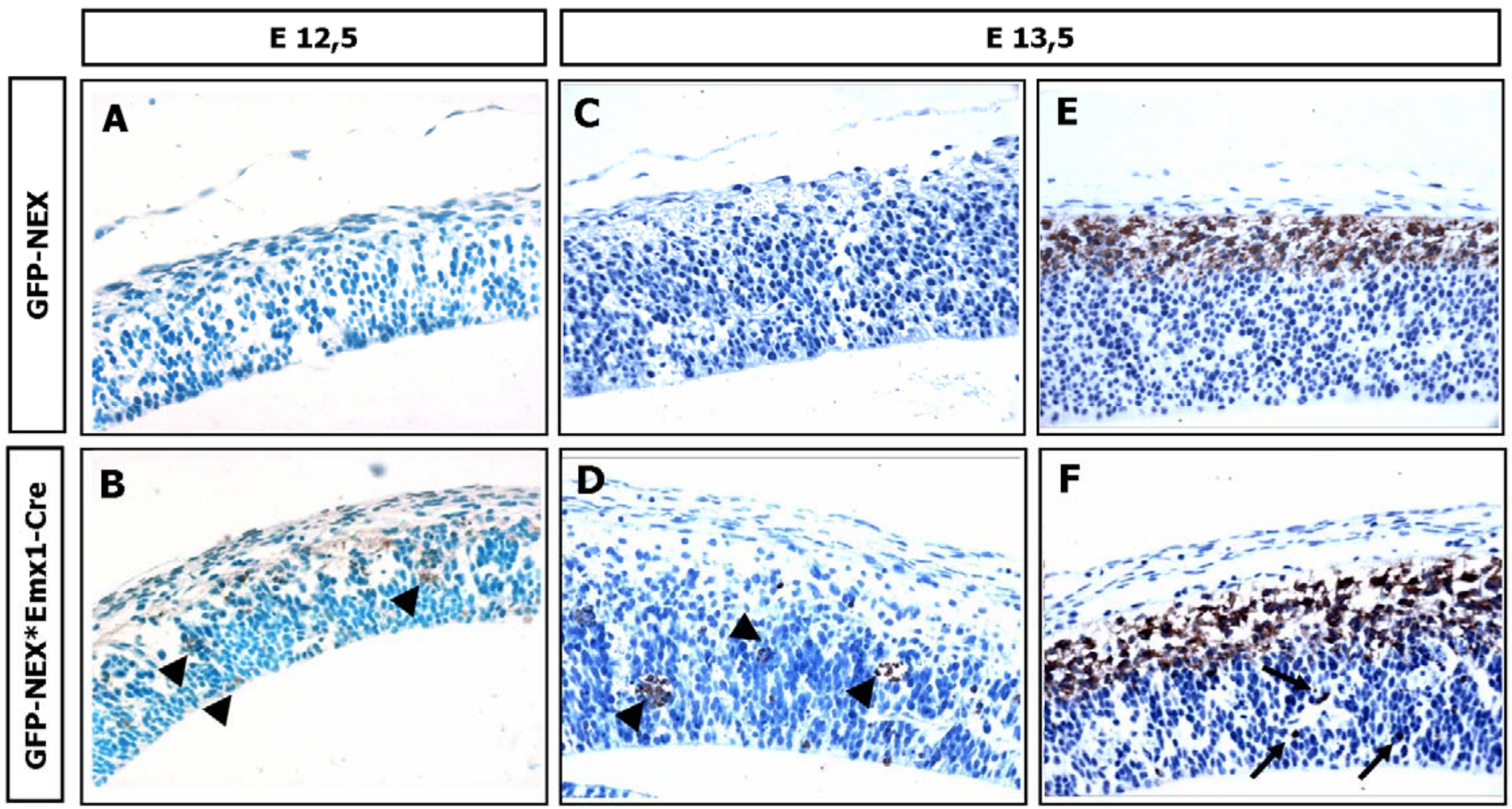

Fig. 19 Increased cell death and ectopic neuronal differentiation in GFP-NEX*Emx1-Cre transgenic mice

Increased apoptosis was revealed by TUNEL immunostaining in the ventricular zone of GFP-NEX*Emx1-Cre mice (B,D) compared to controls (A,C) at E12,5 and 13,5, respectively. Arrowheads in B and D indicate TUNEL-labelled apoptotic cells. Neuronal $\beta$-Tubulin III (Tuj1) immunostaining of control (E) and mutant (F) E13,5 embryonic forebrain revealed premature differentiation in the ventricular zone of the mutants (arrows in D indicate Tuj1-positive cells in the ventricular zone).

(sections were counterstained with Nissl) 


\section{Generation and analysis of NEX-htTA transgenic mouse line}

Many studies of brain function concentrate on the mechanisms of determination, differentiation, maintenance and plasticity of pyramidal neurons. In this context, gain- or loss-offunction experiments of several genes of interest explicitly in the pyramidal neurons (but not in other cell types) of the mouse telencephalon would be invaluable to reveal the mechanisms of neocortex formation in the embryonic development and of neuronal plasticity and higher cognitive functions in the adult animals. The unique expression pattern of NEX, restricted to pyramidal cells in the neocortex, and the fact that the Nex locus has been proven suitable for the expression of heterologous genes (Goebbels et al., 2006) suggest the usage of Nex gene for transgene expression in postmitotic pyramidal neurons. In the course of this study a new transgenic mouse line was created that allows for the inducible overexpression of a transgene under regulatory sequences of the Nex gene. The principle of reporter gene induction was based on the Tet-off system, in which the tetracycline-dependent transactivator (tTA) drives gene expression by binding to the tet-operator (tetO). tTA activity can be efficiently shut down by doxycycline (a tetracycline derivative), that prevents tTA binding to the tetO promoter. In case of the NEX-htTA line a humanized version of tTA (Kim, 2001) was used that is considered to be better tolerated by mammalian cells

\subsection{BAC-based transgene construction and verification of transgene integrity}

In order to increase the probability of faithful recapitulation of the endogenous NEX expression pattern, a bacterial artificial chromosome (BAC) transgenic approach was used for the creation of the NEX-htTA mouse line. The open reading frame (ORF) of NEX on a RP23268011 BAC was replaced by the htTA ORF by means of homologous recombination in bacteria (Fig. 20A; see Copeland et al., 2001). Of 13 BAC-harbouring transgenic founders, only one showed htTA activity after breeding to a reporter mouse line that expresses $\beta$-gal under control of the tetO-promoter. In this line, integrity of the Nex-htTA cassette was verified by PCR on genomic tail DNA (Fig. 20B). RT-PCR on total RNA isolated from cortex and hippocampus of adult transgenic mice demonstrated htTA expression in both brain regions (Fig. 20C). This line was taken for further analysis of htTA-mediated gene expression. 


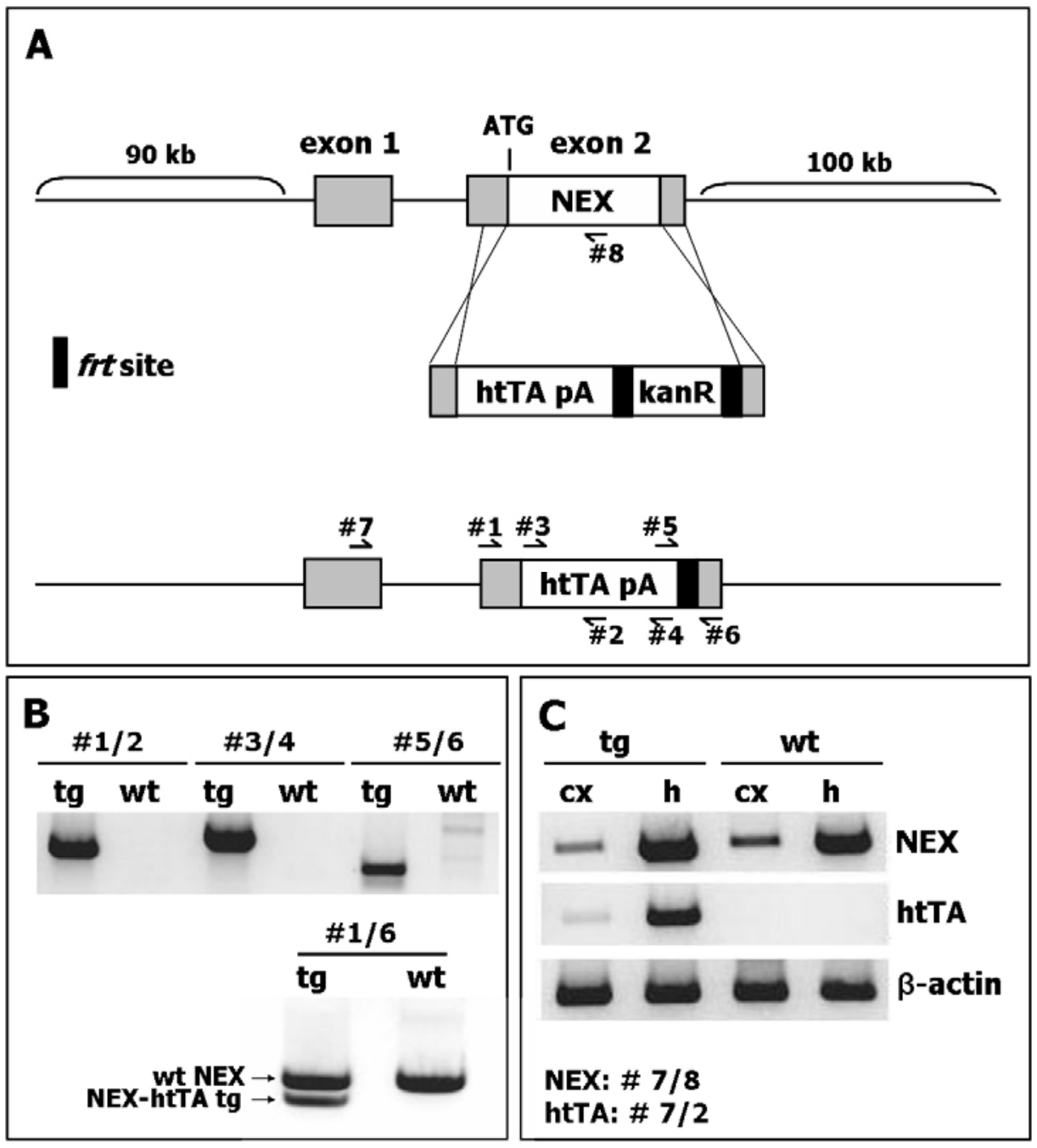

Fig. 20 Schematic representation of NEX-htTA homologous recombination; analysis of transgene integrity and function

(A) Schematic drawing of $190 \mathrm{~kb}$ BAC, harbouring Nex genomic region, and the htTA fragment used for homologous recombination in bacteria (upper panel). Lower panel represents the final transgene construct. PCR with various primers was performed on tail DNA to demonstrate the transgene integrity (B). Note that the primer pair 1 and 6 also amplifies a fragment of the Nex genomic locus. To demonstrate htTA expression, RT-PCR was performed on total RNA isolated from cortex (cx) and hippocampus (h) of transgenic (tg) and wildtype (wt) adult mice (C). (pA - polyadenilation site; kanR - kanamycin resistance cassette; \#1-8 - location of PCR primers used for PCR and RT-PCR analysis, represented in B and C).

\section{2 $\beta$-galactosidase reporter expression pattern}

To examine htTA activity in embryonic and adult brain the NEX-htTA mouse line was initially crossed to a reporter line (GFPG3), harbouring eGFP and $\beta$-galactosidase reporters under the control of a bidirectional tetO promoter (Krestel et al., 2001). X-gal histochemistry was performed on single and double transgenic embryos (whole-mount) and on vibratome sections of adult brain. In double transgenic animals the onset of reporter expression was detectable already at E12 (Fig. 21D). At E13 $\beta$-galactosidase activity was detected in the dorsal telencephalon, cerebellar anlage and spinal cord of NEX-htTA*GFPG3 double transgenic animals (Fig. 21A). In contrast, in single transgenic animals from the same litter X-gal staining 
was almost completely absent (Fig. 21B). Doxycycline administration to the pregnant mother efficiently suppressed htTA-dependent reporter expression, since no $\beta$-galactosidase activity was observed in double transgenic embryos that received doxycycline treatment (Fig. 21C).
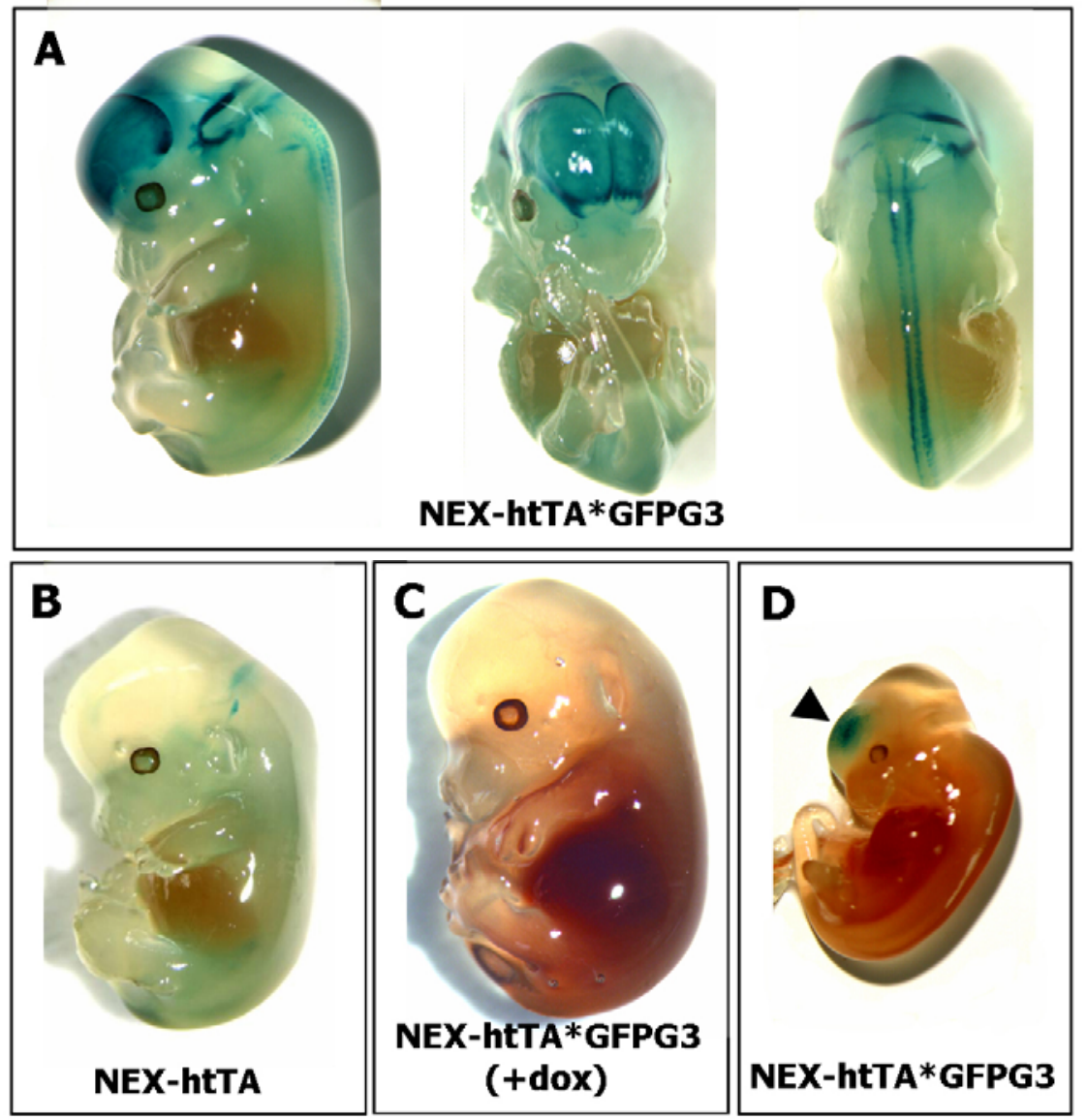

Fig. 21 NEX-htTA mediated lacZ reporter expression in embryonic development

A double transgenic NEX-htTA*GFPG3 embryo at E13,5 shows X-gal staining in the telencephalon and spinal cord (A), whereas no X-gal signal is observed in a single transgenic NEX-htTA embryo from the same litter (B). Similarly, no X-gal staining was observed in a double transgenic NEX-htTA*GFPG3 embryo at E14 when pregnant mother has received doxycycline throughout pregnancy (C). Double transgenic NEX-htTA*GFPG3 embryos exhibited X-gal staining in the forebrain already at E12 (D).

Because of the reported unreliability of transgenic $\beta$-galactosidase expression in several mouse lines (Robertson et al., 2002; Chevalier-Mariette et al., 2003; T. Fischer, personal communication), a Cre recombinase-based reporter approach was used to investigate htTAdependent gene expression. For this, a reporter line was used that harbours a stop-cassette 3 ' to $\beta$-galactosidase ORF in the Rosa26 locus and thus allows monitoring of Cre recombinase activity by Xgal staining (Soriano et al., 1999). To generate triple transgenic animals, NEX-htTA mice were crossed to tetO-Cre*Rosa26 (Schonig et al., 2002; Soriano et al., 1999), and $\beta$ galactosidase activity was visualised in the offspring by X-gal staining of the adult brain. Triple transgenic animals exhibited X-gal staining in cerebral cortex, hippocampus, amygdala, piriform and entorhinal cortex and supramamillary bodies (Fig. 22A). Administration of doxycycline successfully suppressed $\beta$-galactosidase expression in triple transgenic mice (Fig. 22B). 


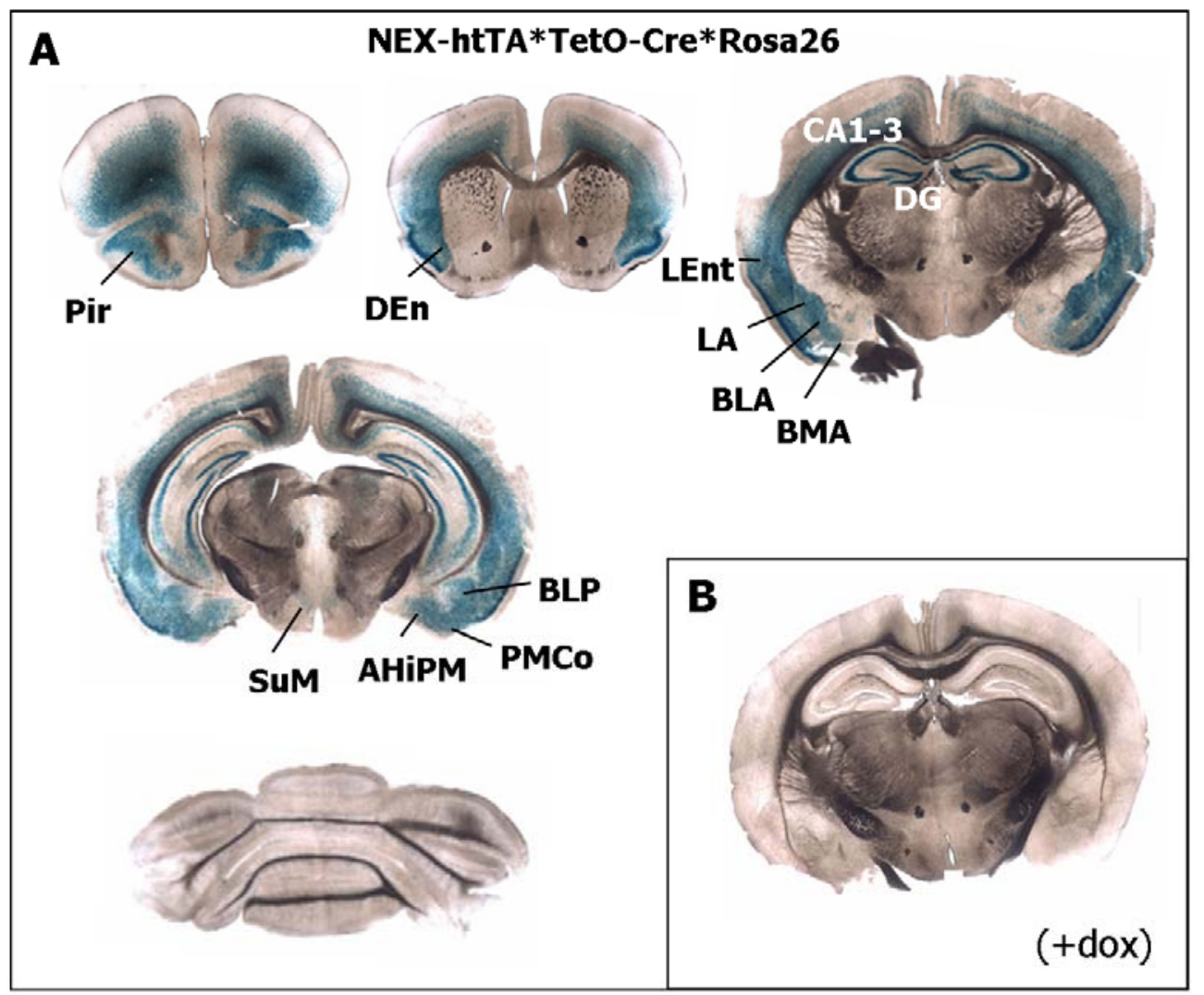

Fig. 22 LacZ reporter expression in the brain of adult NEX-htTA*tetO-Cre*Rosa26 mice

For evaluation of reporter activity, $100 \mu \mathrm{m}$-thick vibratome serial brain sections of triple transgenic animal (NEXhtTA*tetO-Cre*Rosa26) at P30 were stained for X-Gal (A). No lacZ activity is observed if the mouse has received doxycycline (B).

(AHiPM, amygdalohippocampal area, post-med; BLA, basolateral amygdaloid nu, anterior; BLP, basolateral amygdaloid nu, posterior; BMA, basomedial amygdaloid nu, anterior; CA1-3, fields CA1 to 3 of hippocampus; DEn, dorsal entopiriform nu; DG, dentate gyrus; LA, lateral amygdaloid nucleus; LEnt, lateral entorhinal cortex; PMCo, posteromedial cortical amygdaloid nucleus; SuM, supramammillary nucleus)

Surprisingly, in the cortex of NEX-htTA* tetO-Cre*Rosa26 animals $\beta$-galactosidase was only detected in deeper layers, in contrast to the recombination pattern induced by Cre expression directly from the Nex genomic locus (Goebbels et al., 2006). Combination of X-gal histochemistry with $\alpha-\mathrm{NeuN}$ immunostaining clearly demonstrated that the majority of X-gal positive cells was located in layers V and VI (with some scattered cells in layer IV), whereas cells in cortical layers II-III virtually lacked $\beta$-galactosidase activity (Fig. 23A,B).

X-gal histochemistry on coronal brain sections of NEX-htTA*GFPG3 double transgenic mice at P5 revealed a reduction of cortical $\beta$-galactosidase activity. Only very few X-gal positive cells were present in the cortex (Fig. 24A), however, hippocampus and entorhinal cortex showed continuous reporter expression. Further analysis of NEX-htTA transgene activity in postnatal brain was done using NEX-htTA* tetO-Cre*Rosa26 triple transgenic animals, that had received doxycycline treatment. In order to suppress htTA activity during embryonic development, doxycycline was administered to the pregnant mother till birth. The kinetics of doxycycline has 
not been efficiently studied in embryonic and early postnatal brain (K. Schoenig, personal communication), however, the data obtained on adult animals suggest that the onset of reporter expression takes place 1-2 weeks after the end of doxycycline treatment (Sprengel and Hasan, 2007).

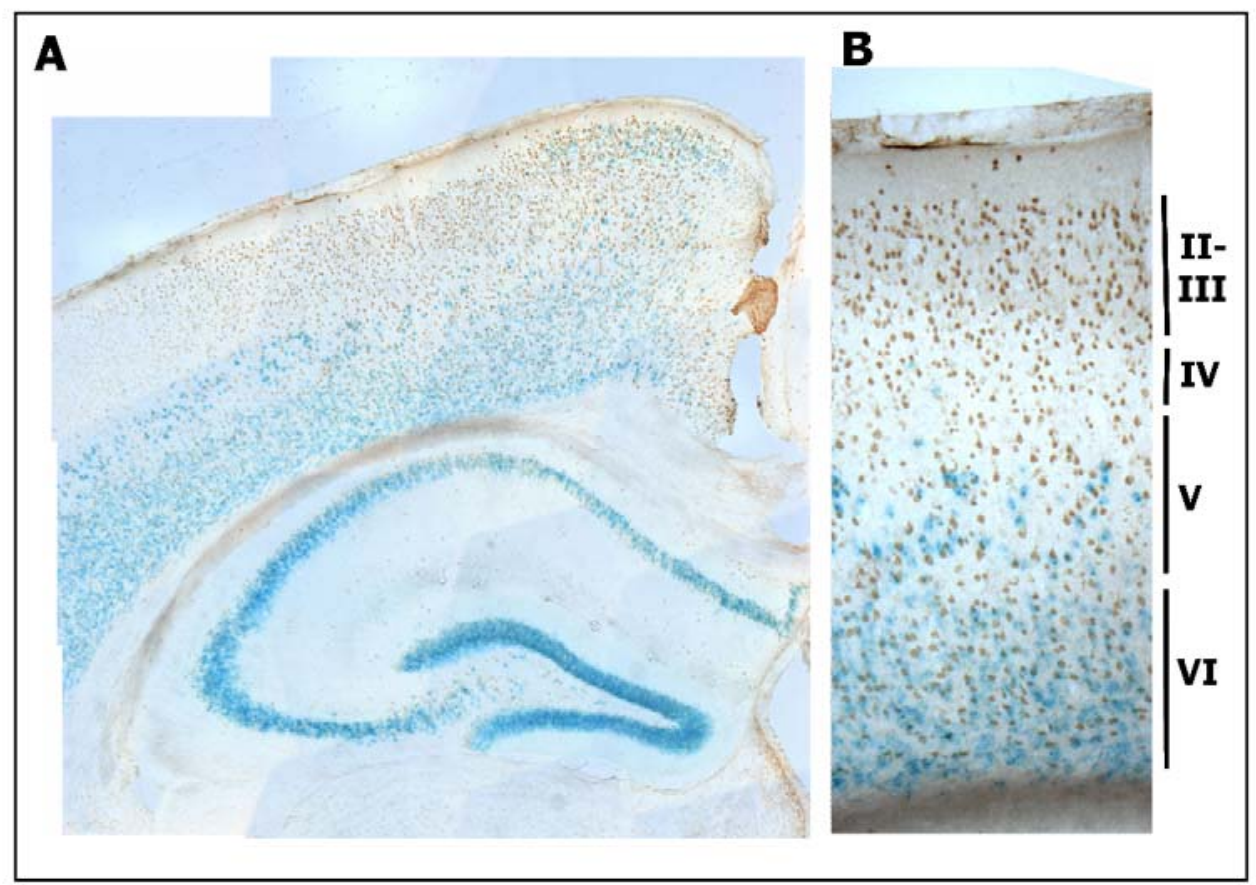

Fig. 23 NEX-htTA*tetO-Cre* Rosa26 transgenic mice reporter expression is restricted to cortical layers $\mathrm{V}$ and VI

X-gal staining of $100 \mu \mathrm{m}$-thick vibratome sections of the brain from a NEX-htTA*tetO-Cre*Rosa26 triple transgenic mouse was combined with $\alpha-\mathrm{NeuN}$ immunohistochemistry (A). X-gal positive cells are present in deeper (V and VI) cortical layers (B).

Therefore, after doxycycline withdrawal at P0 the mice were sacrificed 6 or 8 weeks later, and X-gal histochemistry on coronal brain sections was performed. Despite the prolonged clearance period, these animals displayed virtually no $\beta$-galactosidase activity in the cerebral cortex (Fig. 24, B). X-gal positive cells were detected in dentate gyrus, the CA3 region and entorhinal cortex (Fig. 24C,D). Similar results were obtained when the dosage of doxycycline given to the pregnant mother was decreased from $100 \mathrm{mg} / 1$ to $25 \mathrm{mg} / \mathrm{l}$.

An overview of X-gal staining patterns in different reporter lines is presented in Tab. 6 . The pattern of $\beta$-galactosidase activity in the postnatal brain was restricted to only a subset of pyramidal neurons in both GFPG3 and tetO-Cre*Rosa26 reporter mouse lines. Ongoing detailed investigation of reporter expression during forebrain development and doxycycline kinetics studies will help to reveal the exact spatiotemporal pattern of htTA activity in the NEX-htTA transgenic mouse line. 


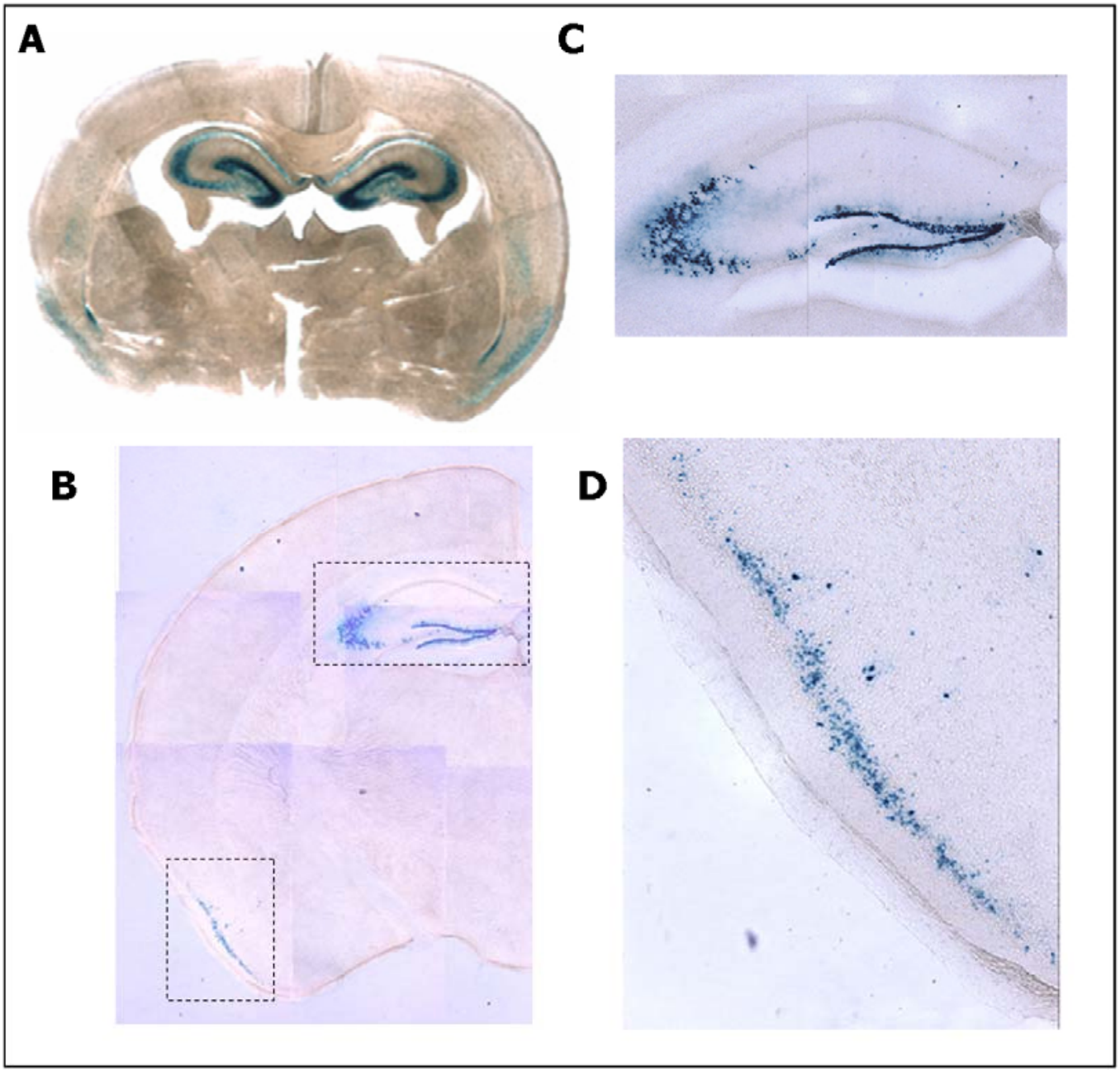

Fig. 24 htTA drives reporter expression only in a subset of pyramidal neurons in the postnatal brain X-gal staining of a coronal brain section of NEX-htTA*GFPG3 double transgenic mouse at P5 revealed strongly reduced reporter expression in the neocortex (A). For evaluation of postnatal Cre activity, X-gal staining was performed on NEX-htTA*tetO-Cre*Rosa26 triple transgenic animal that had received doxycycline treatment until birth. Reporter activity was restricted to the hippocampus and entorhinal cortex (B). (C, D) Blowup of boxed areas in $\mathrm{B}$.

Tab. 6 X-gal staining of different reporter lines, crossed with NEX-htTA transgenic line

\begin{tabular}{|l|c|c|c|}
\hline \multicolumn{1}{|c|}{ Area } & GFPG3 & tetO-Cre*Rosa26 & $\begin{array}{c}\text { tetO-Cre*Rosa26 } \\
(+ \text { dox till birth })\end{array}$ \\
\hline telencephalon E12 & + & + & n.a. \\
\hline telencephalon E13,5 & + & + & n.a. \\
\hline Adult brain: & & & few cells \\
\hline neocortex & few cells & deeper layers & - \\
\hline CA1 & + & + & + \\
\hline CA3 & + & + & + \\
\hline dentate gyrus & + & + & few cells \\
\hline amygdala & few cells & + & + \\
\hline entorhinal cortex & + & + & \\
\hline
\end{tabular}




\section{DISCUSSION}

The goal of this thesis was to investigate the role of the bHLH transcription factor NEX during development and in the adult brain. As discussed below, the analysis of developmental and adult functions of NEX was separated into two parts. The first part of this study comprised the profiling of gene expression in the hippocampus of adult NEX null mutants versus wildtype mice using DNA microarrays and a detailed analysis of behavioural changes in the mutant. Since NEX null mutants lack an obvious developmental phenotype, this section addresses the function of NEX in the adult brain. In the second part of this thesis the function of NEX during development was investigated in a gain-of-function approach. For this purpose a transgenic mouse line was generated that allows an inducible and cell type-specific overexpression of NEX. In a pilot study NEX was overexpressed in neuronal progenitors of the murine telencephalon in order to reveal a possible role of NEX in control of neural progenitor cell cycle and neuronal differentiation. Finally, a second transgenic mouse line was produced and characterized that expresses htTA under control of the NEX promoter. This mouse line will be discussed as a tool to study cortical development.

\section{The effect of NEX deficiency on hippocampal gene expression in the adult mouse}

The expression pattern of NEX has been previously described at various stages of embryonic development and in the postnatal mouse brain (Schwab et al., 1998). High levels of NEX mRNA are detected in principal neurons of the cortex, CA1-3 regions of the hippocampus, subiculum and amygdala. This expression pattern suggested a role for NEX in the development and plasticity of principal projection neurons, however, the development of the telencephalon appears to be largely unaffected in NEX null mutant mice (Schwab et al., 1998).

NEX belongs to the NeuroD family of neuronal differentiation factors. High structural homology and largely overlapping expression domains of NEX, NeuroD and NDRF suggest a possible partial functional redundancy between members of the NeuroD family (Schwab et al., 1998; Schwab et al., 2000). In general, functional redundancy during nervous system development appears to be a prominent feature of bHLH proteins (Inoue et al., 2002; Ohsawa et al., 2005; Kruger et al., 2006).

Functional redundancy during development does not exclude functional divergence in the adult brain. In order to identify factor-specific functions, expression overlap has also to be taken into account in functional studies of closely related factors. NEX mRNA levels are higher than those of NeuroD and NDRF in the CA3 region of the hippocampus, the entorhinal cortex and the 
subiculum (Schwab et al., 1998; M. Gummert, unpublished observation). Since physiological properties, cellular composition and molecular profiles have been best described for the CA3 region of the hippocampus, it was chosen for a microarray analysis of NEX null mutants.

A promising way to investigate a possible role of a transcription factor in regulating neuronal function and plasticity is to perform a molecular and physiological analysis of the brain in situations that induce plasticity changes. The enriched environment paradigm is such a positive challenge; indeed, exposure of rodents to an enriched environment has been shown to increase dendritic branching and length, number and size of synapses in certain populations of neurons, hippocampal neurogenesis and functional integration of newborn neurons (van Praag et al., 2000; Nithianantharajah and Hannan, 2006). The identification of molecular changes in the rodent cortex and hippocampus that are triggered by the exposure to an enriched environment have demonstrated a clear relationship between environmental complexity and changes in the expression profile of these brain areas (Rampon et al., 2000; Ronnback et al., 2005). In this study the enriched environment paradigm was applied and the potential consequences of the NEX null mutation on enrichment-induced changes in neuronal plasticity and metabolism was assessed. The duration of exposure to the enriched environment was set at 2 and 7 days in order to increase the probability to investigate the dynamic "onset phase" of NEX-dependent changes of gene expression upon environmental enrichment.

\subsection{Expression profiling of the $\mathrm{CA3}$ region of the adult hippocampus}

Previously published studies regarding the expression of NEX have focused on embryonic and early postnatal stages (Schwab et al., 1998) and did not fully answer the question of whether expression is maintained throughout the entire life of the mouse. Here, it was shown by in situ hybridization that NEX mRNA expression in the adult mouse brain is maintained in the CA1-3 regions of the hippocampus at least until 12 months of age (Fig. 1A), in contrast to NeuroD, whose expression has been shown to severely decline in the aging hippocampus (Uittenbogaard and Chiaramello, 2000).

For the comparison of CA3 expression profiles of NEX null mutants and wildtype mice, laser-capture microdissection of the CA3 region was performed. To minimize the influence of circadian rhythms on gene expression, mice were sacrificed at the same time of the day and the dissected brains were immediately frozen on dry ice. Sectioning was performed at $-20^{\circ} \mathrm{C}$. This procedure assures preservation of RNA integrity and is essential for microarray analysis. The tissue was isolated from thionin-stained unfixed sections as demonstrated in Fig. 2B-D. The hilar region was excluded from the laser-dissected area, which additionally lowered the cellular diversity of the analysed material. A three-round RNA amplification method, which was used in 
this study, has been described previously as highly suitable for small RNA quantities (Rossner et al., 2006). The size distribution of the probes (Fig. 2E) demonstrated that high probe quality was achieved. To determine the effect of technical variation on the experimental outcome, two technical replicas were prepared for each probe.

\subsection{Gene expression changes in wildtype mice upon environmental enrichment}

Expression profiling of enriched environment-induced transcriptional changes has been performed previously; however, in the reported experiments the profiles of large brain areas (i.e. cortex, hippocampus) or even the total brain were assessed (Rampon et al., 2000; Ronnback et al., 2005). The obtained transcriptional profiles normally do not provide information about expression changes in smaller groups of neurons (Lein et al., 2004). In contrast, the method of high-precision laser-capture microdissection (LCM) allows investigating transcriptional changes in specific populations of cells. Thus, this approach is more likely to elucidate the effect of environmental enrichment on the metabolism, maintenance and plasticity of specific cell types. Microarray analysis provides information about all transcriptional changes, therefore, this method allows much more detailed insights into changes of virtually all cellular processes, when compared to any gene-by-gene analysis such as immunohistochemistry or in situ hybridization (Pinaud et al., 2001; Pinaud et al., 2002; Lein et al., 2004).

In addition to the regular microarray profiling, which typically provides a list of differentially regulated candidate genes, a "gene set enrichment" analysis (GSEA; Mootha et al., 2003; http://www.broad.mit.edu/ personal/aravind/GSEA/index.html) was performed. This method of analysis focuses on groups of genes (gene sets) that share common biological function, chromosomal location or regulation. The results of GSEA are easier to interpret in terms of biological function than the conventional single gene scoring; indeed, a $20 \%$ expression change in several components of a metabolic pathway can often tell more than a 20 -fold increase in a single gene expression (Subramanian et al., 2005). In this study GSEA revealed an upregulation of metabolic and signalling pathways in the CA3 region of wildtype animals upon environmental enrichment (see Fig. 3; Tab.2), indicating increased neuronal activity and plasticity in the challenged animals. Interestingly, these transcriptional changes follow a certain order, such that shifts in metabolic activity (oxidative phosphorylation and electron transport chain gene sets) are already detectable after 2 days of exposure to the enriched environment, while upregulation of signalling cascades (e.g. MAPK cascade), which indicate an increase in plasticity, occurs only after prolonged exposure to the enriched environment (Tab. 2, Fig. 3).

The precise isolation of defined brain areas by LCM also has the advantage of minimizing the risk of contamination by other regions. In several gene expression studies, which 
were performed on crude preparation of hippocampus, transthyretin (ttr) was shown to be upregulated upon environmental enrichment (Ronnback et al., 2005). However, since ttr is highly expressed in the choroid plexus, the possibility of a contamination could not be excluded (Barlow and Lockhart, 2002; Lein et al., 2004; Ronnback et al., 2005). Changes in ttr expression have also been detected in this study (Fig. 6C, D). Since a contamination with choroid plexus during LCM of the CA3 region is unlikely, this observation suggests that changes in hippocampal $\mathrm{ttr}$ expression indeed occur as a response to environmental enrichment. A more detailed analysis of enriched environment exposure-mediated transcriptional changes in wildtype animals, including the CA1 region, is ongoing and will provide more detailed information about neuronal plasticity processes induced by differential housing of mice.

\subsection{Metabolic changes in NEX null mutants indicate hyperactivity}

A comparison between gene expression profiles of NEX null mutants and wildtype controls, housed in regular cages, identified alterations in the transcription of several functional groups of genes (Fig. 4), including those involved in transcriptional regulation, mitochondria function, cell cycle, cell growth and intracellular signalling. A gene set enrichment analysis revealed a shift in oxidative phosphorylation and the diacylglycerol pathway in mutant mice (Fig. 4), which, together with differential expression of mitochondrial proteins, indicates an increased metabolic activity in null mutants. Mitochondria are tightly associated with neuronal plasticity, both severe (stroke models) and moderate (enriched environment exposure) metabolic stress induce changes in mitochondrial protein composition and increase the number of mitochondria in synapses (Mattson, 2007). The observation of metabolic stress in NEX null mutants, housed in regular cages, suggests that mutants exhibit increased physical activity than their wildtype littermates. Interestingly, the level of calbindin mRNA was 20 fold higher in the CA3 region of null mutants (Fig. 6A; Tab. 3). An increase in calbindin indicates higher locomotory activity (Airaksinen et al., 1997), however, since calbindin has not been shown to be expressed in pyramidal neurons of the CA3 region, this might be an indirect effect of the NEX null mutation on other cell types.

Several cytoskeleton-related genes were differentially expressed in NEX mutants (Tab. 3, 4, 5). Interestingly, expression of some cytoskeletal proteins (e.g. Tmsb10, Hook1 and Marcks) was upregulated in null mutants, housed in regular cages, while exposure to the enriched environment resulted in downregulation of other cytoskeleton-related genes (Acta2, Ncald, Myl6, Epb4.112 and Scin) in the mutants. Such a shift in the pool of cytoskeletal proteins is compatible with plasticity changes in neurons (Dillon and Goda, 2005). In line with this hypothesis, several genes coding for synaptic proteins and ion channels were found to be 
differentially expressed in the null mutant at different time points of environmental enrichment (Tab. 3-5). For instance, expression of Doc2b, Stxbp6, Arhgap20 was higher in the CA3 hippocampal region of NEX null mutants during regular housing, but not upon environmental enrichment. In contrast, the expression level of Cacng5 and Cadps2 was higher in wildtype controls after 7 days of enriched environment exposure. A great part of differentially regulated genes are components of intracellular signalling pathways (Fig. 4, 5), that might mediate plasticity changes in neurons. In particular, Ptprz1, Cnih3, Dusp14, Plekha2, Pcsk5, Camk1, Inpp4b, Pcsk5 and Trib2A were upregulated in the null mutants compared to controls at different time points, whereas the expression of Ctsc, Adcy1, Phb2, Dusp5, Sostdc1, Frzb and Gng4 was downregulated. The misregulation of genes involved in intracellular signalling and synaptic plasticity in NEX null mutants indicates defects in neuronal function in the CA3 hippocampal region of these animals, however, the exact molecular mechanisms involved are still unclear and require more detailed analysis of gene expression data.

Interestingly, the expression of some transcription factors, e.g. Pou3f1 and Egr2, was differentially regulated in null mutants and wildtype animals, housed in both regular and enriched cages, suggesting a role of NEX in the regulation of molecular mechanisms involved in axonal outgrowth and myelination control. The fact that Pou3fl expression level is higher in the CA3 region of NEX mutants compared to controls in regular cage housing (Tab. 3), but vice versa profile is observed upon environmental enrichment (Tab. 4,5), indicates the involvement of NEX in the dynamic changes of Pou3f1 expression. Investigation of NEX-mediated transcriptional control of myelination-related genes, including profiling of the mutant CA1 region, will help revealing the link between neuron-glia interaction and mouse cognitive functions.

Finally, several genes involved in higher cognitive functions were differentially regulated in NEX null mutants and wildtype controls. Penk1 (pre-proenkephalin), involved in anxiety behaviour (Bilkei-Gorzo et al., 2004), showed an increased expression level in null mutants housed in regular cages. Fos, an immediate early gene involved in cognitive processes, was downregulated in mutants housed in regular cages (Tab. 3), but no difference in Fos expression was observed between mutants and controls after exposure to the enriched environment. This suggests that NEX contributes to the transcriptional control of basal Fos expression, while the enrichment-induced upregulation of Fos is NEX-independent. In contrast, Prkar2b, a protein kinase A subunit, was upregulated in the mutants, both under regular and enriched conditions (Tab. 3-5). Prkar2b null mutants exhibit locomotory deficits and altered gene expression (Brandon et al., 1998); moreover, protein kinase A is a well described component of the signalling cascades involved in learning and memory formation (Impey et al., 1998). Increased 
level of Prkar2b mRNA in NEX mutants indicates a deficit in the regulation of these signalling cascades.

Transthyretin (ttr), a component of the retinol transport pathway, showed an environmental enrichment-dependent expression profile both in wildtype mice and null mutants, the expression level being much lower in the mutants than in the wildtype mice after 2 days of environmental enrichment. However, after 7 days of enriched environment exposure ttr expression level was higher in the mutants than in the wildtype animals (Fig. 6C,D). Ttr has been implicated in hippocampus-dependent memory formation and maintenance in aged rodents (Brouillette and Quirion, 2007). An increase in ttr and ptgds (prostaglandin D2 synthase) expression in the hippocampus is associated with memory consolidation (Levenson et al., 2004). In this study the expression level of ptgds stayed constant in wildtype mice, but was upregulated in null mutants after 2 days of complex cage housing (Tab. 4).

Differential gene expression in NEX null mutants versus wildtype mice implies synaptic plasticity, behaviour and memory defects in the mutants. Several behavioural assays that will be discussed in the following chapters have confirmed this hypothesis.

\section{Behavioural analysis of NEX mutants}

\subsection{NEX null mutants exhibit hyperactivity and learning deficits}

NEX null mutants showed normal basic anxiety and fear levels when compared to wildtype mice in the open field (Fig. 8A), light-dark preference (Fig. 9A) and plus maze (Fig. 9B) tests. However, they exhibited hyperactivity and higher velocity, resulting in longer total distance travelled in the open field (Fig. 8E-H) and an increased number of crossings between compartments in the light-dark box and plus maze (Fig. 9C,D). The increased latency of NEX mutants to fall off in the Rotarod motor learning task (Fig. 7D), can also be attributed to hyperactivity and higher locomotion speed. Average swimming speed measured in the Morris water maze was also significantly elevated in the mutants (Fig. 10A). Together with the microarray data, these results indicate that increased metabolic activity in the CA3 hippocampal region is associated with higher locomotory activity in the null mutants. Importantly, the results obtained from light-dark preference and plus maze tests demonstrate that this increase is not associated with elevated anxiety (see Fig. 9A,B). Interestingly, a mutation in NDRF, a transcription factor closely related to NEX, results in decreased anxiety and emotional learning deficits of heterozygous mutant mice (Lin et al., 2005). The fact that NEX mutants display normal anxiety and fear levels supports the hypothesis of divergent roles for NEX and NDRF in the adult mouse brain. 
Hippocampus-dependent spatial memory formation was tested in the Morris water maze. NEX null mutants demonstrated slower learning in the hidden platform task (Fig. 10C; Fig. 11A) and a spatial memory deficit in the probe trials (Fig. 10D; Fig. 11C,D). However, the mutants seem to master the hidden platform task, as demonstrated by the constant decrease of the travelled distance (Fig. 10C; Fig. 11A).

The outcome of water maze training largely depends on the training paradigm. Detailed analysis of the performance of both experimental cohorts in the visible and hidden platform training demonstrates that the control mice require less time to reach the visible platform if the visible platform training is preceded by hidden platform training (Fig. 12A,B). In this paradigm, NEX null mutants also efficiently mastered the task (Fig. 12B). However, when the visible platform task was placed at the beginning of the training, a significant difference between null mutant and control mice was observed on the second day (Fig. 12A).

Learning in the Morris water maze task includes explicit and implicit components. The spatial learning component results in the formation of explicit memory of platform location; progress in this task is reflected by a decrease in the latency to reach the platform on consecutive training days and a preference for target quadrant in the probe trial (Crawley, 2000). However, an implicit memory component is also required for successful spatial memory formation, since the mouse has to learn the motor task of swimming to the platform and to associate the platform with escape. This implicit component has not been studied in detail; rather, it has been largely ignored in water maze-based tests (McGaugh, 2005; Rossato et al., 2006).

The poor performance of NEX null mutants in the probe trials (Fig. 10D; Fig. 11C,D) demonstrates that the explicit component of memory involved in mastering the water maze task, i.e. the ability to remember the platform location, is disturbed in the mutants. There is also an indication that the implicit component of learning might be disturbed. Mutants display poor learning on the second day of the relatively easy visible platform task (Fig. 12A), but are not different from wildtype animals if the task is performed after the hidden platform task (Fig. 12B). The inability of NEX null mutants to associate the platform (visible or hidden) with the means of escape (or a certain delay in this association) can partially account for their lag in spatial learning in the hidden platform task. The trial-by-trial analysis of performance on the first day of the hidden platform task demonstrates that wildtype mice travel shorter distance after being shown the location of the platform, whereas NEX null mutants fail to improve (Fig. 12C). This lack of improvement in trials performed within approximately 1 hour might indicate an impairment of either spatial short-term memory or motivation (i.e. association of the platform with escape). 
Another indication that decision making might be impaired or delayed in the mutants was derived from the hot plate test. The latency to lick the hind paw in the mutants is slightly (but not significantly; $p=0,057$ ) higher than that of wildtype control animals (Fig. 7C). Although the hot plate test is generally viewed as a pain sensitivity test, it includes a cognitive component. Unlike tail immersion or tail flick tests that measure the physiological events occurring at spinal cord level, the hot plate response involves events that are integrated at higher brain levels (Kieffer, 1999). The tendency for increased latency observed in NEX null mutants suggests that this integration might be slightly delayed; however, the results presented here are inconclusive. Altogether the learning deficit displayed by mutants in the Morris water maze seems to consist of more than one cognitive component and suggests further behavioural studies.

\subsection{NEX deficiency has differential effects on recent and remote memory}

Differences in molecular mechanisms and brain structure involved in recent and remote memory formation, reconsolidation and extinction (Lee et al., 2004; Maviel et al., 2004; Suzuki et al., 2004; Frankland and Bontempi, 2005; Squire and Bayley, 2007) indicate the presence of various control mechanisms responsible for memory formation and the importance of connections between different brain areas. The expression pattern of NEX (Schwab et al., 1998) suggests an involvement in maintenance and proper functioning of the entorhinal cortex, supramamillary bodies and subiculum, which are known to be involved in the interplay between the hippocampus and association areas of the cortex (de la Prida et al., 2006; Yasuda and Mayford, 2006). The fear conditioning paradigm was chosen to assess different types of memory formation and extinction in NEX null mutant mice.

Fear memory assessment revealed a unique phenotype in NEX null mutants. Although null mutants demonstrated high levels of freezing response in the cue memory assessment (Fig. 13A), indicative of undisturbed formation of hippocampus-independent recent fear memory, their contextual (hippocampus-dependent) recent fear memory was greatly reduced compared to wildtype (Fig. 13B). In contrast, their remote contextual memory was not disturbed (Fig. 13E). The extinction to context, performed at short (1 day) and long (36 days) retention times, showed a reverse pattern: null mutants demonstrated virtually no reduction in freezing response in recent memory extinction (Fig. 13C,D), but a significantly faster extinction was observed for remote contextual memory in mutants compared to controls (Fig. 13F).

In order to analyse the specific memory deficits displayed by NEX null mutants, their deficits in memory acquisition, reconsolidation and extinction will be discussed independently. 


\subsubsection{Fear memory acquisition}

The acquisition (consolidation) of recent contextual fear memory is known to depend on hippocampus, entorhinal cortex and subiculum, however, inactivation of these brain areas, while impairing new memory formation, does not have an impact on already recorded memories (Clark et al., 2002; Maviel et al., 2004; Yasuda and Mayford, 2006). The current theory of systems consolidation suggests a temporal role for the hippocampal formation in memory storage. According to this theory spatial memory is transferred within days through a hippocampocortical network to storage areas in the cortex (Frankland and Bontempi, 2005).

NEX null mutants display a normal level of freezing in the recent cue (tone) memory assessment, a task that involves cortex and amygdala, but does not depend on hippocampus (Sanders et al., 2003). Their reduced fear response to the context in the recent memory test indicates a hippocampus-specific memory deficit. In line with the hypothesis that hippocampal, but not cortical function is disturbed in the mutants, no deficit in remote contextual memory was observed. High levels of NEX expression in hippocampus, entorhinal cortex and subiculum (Schwab et al., 1998) imply that the proper function of one or all of these areas is disturbed in the mutant, resulting in the specific behavioural phenotype observed in the fear conditioning paradigm. Although NEX is expressed in pyramidal neurons throughout cortex and amygdala, normal cue memory and remote contextual memory, displayed by NEX mutants, imply that NEX expression in amygdala or the prefrontal cortex is not required for memory acquisition processes that do not depend on hippocampus or hippocampo-cortical connections.

The specific defect of recent, but not remote memory recall in NEX null mutants resembles that observed in studies of lesions or temporal inactivation (e.g. by infusion of a protein synthesis inhibitor) of the hippocampus (Clark et al., 2002; Maviel et al., 2004; Yasuda and Mayford, 2006). However, the situation in NEX null mutants is different from these experiments. For instance, the absence of NEX is permanent, in contrast to temporally restricted inhibition. Pre-training hippocampal lesions, if performed at long delay before training, have little or no impact on fear memory formation (Heldt et al., 2002), suggesting that although hippocampus is normally required for contextual representation, neocortex can perform this role in its absence (O'Reilly and Rudy, 2001; Sanders et al., 2003). In a previously proposed model hippocampus, when intact, inhibits the neocortex from forming a redundant representation of the context; if hippocampus is inactivated long enough before training, the inhibition is released and allows acquisition of normal contextual fear memory, mediated by the neocortex alone (Fanselow, 2000; Sanders et al., 2003). The failure of neocortex to overtake the contextual representation function in NEX null mutants demonstrates the presence of hippocampus- 
mediated inhibition of the neocortex in these animals; therefore, the lack of NEX does not cause a complete inactivation of hippocampal function.

Some insight into the particular hippocampal deficit of NEX null mutants was gained from transcriptional profiling. Thus, ttr, which is implicated in hippocampal fear memory formation (Stein and Johnson, 2002; Tsai et al., 2002), was differentially regulated in NEX null mutants and wildtype mice after environmental enrichment (Fig. 6C,D). An increase in ttr expression following fear conditioning was demonstrated in the CA1 region of the hippocampus (Levenson et al., 2004). Gene expression data from the CA3 region suggest that ttr upregulation in NEX null mutants is not completely absent but rather delayed. Indeed, there is an increase in ttr expression levels in the mutants after 2 days of environmental enrichment (Fig. 6C,D), albeit not quite as high as in control animals. After 7 days of environmental enrichment ttr mRNA level is higher in the mutants than in the controls (Tab. 5, see also Fig. 6C,D). This delayed upregulation of ttr expression might be a molecular indication of a delay (but not complete failure) of hippocampus-dependent processing in NEX null mutants. The delay hypothesis is supported by the lag (but not failure) of hippocampus-dependent learning, observed in the Morris water maze task in this study.

\subsubsection{Fear memory extinction}

Re-exposure to the conditioning context in the absence of the unconditioned stimulus (foot shock) has a dual effect on fear memory in rodents: retrieval (and reconsolidation) of original memory of the context is accompanied by extinction of such memory. Interestingly, original consolidation and retrieval-induced reconsolidation of memory involve different molecular processes. Thus, in the hippocampus consolidation requires BDNF, whereas reconsolidation is dependent on the transcription factor Zif268 (Lee et al., 2004). Upregulation of NGFI-B (nerve growth factor-inducible gene B) accompanies consolidation, but not reconsolidation processes (von Hertzen and Giese, 2005). Although recent memory retrieval is associated with Zif268 and fos upregulation in the hippocampus, recall of remote memory induces an increased expression of these factors in prefrontal and anterior cingulate cortices (Maviel et al., 2004), confirming the theory of memory transfer to cortical networks over time. In line with this observation, remote contextual memory recall has been shown to induce activation of prefrontal cortex, but not hippocampus (Frankland and Bontempi, 2005).

Although initial encoding and reactivation of fear memories require the involvement of different brain areas, they both depend on de novo transcription and protein synthesis (Kida et al., 2002). Reconsolidation is additionally affected by the extinction process, in which the original memory strengthening competes with formation of a novel memory; thus, during 
extinction of conditioned fear the animal learns that the conditioned stimulus (context) is not always associated with the unconditioned stimulus (shock). The duration of re-exposure seems to be the key element of such competition. Studies on reconsolidation and extinction have revealed three key phases of memory processing during re-exposure (Kida et al., 2002; Suzuki et al., 2004). The retrieval of memory happens first and precedes both reconsolidation and extinction. After a certain length of exposure first the reconsolidation process and then the extinction process is triggered. Both reconsolidation and extinction require de novo protein synthesis, and while short re-exposures suffice for memory reconsolidation, longer re-exposure time seems to favour extinction (Suzuki et al., 2004). The two competing processes take place in different brain areas. Reconsolidation of recent fear memory involves amygdala and hippocampus, while extinction learning depends on the medial prefrontal cortex (Quirck et al., 2000; Nader et al., 2000; Debiec et al., 2002).

Older memories are more resistant to extinction, thus, a much longer re-exposure time is required for the disruption of remote compared to recent memory (Suzuki et al., 2004). The explanation for an increased stability of older memories may lie in the transfer of remote memory from the place of original encoding to other brain areas. In this study the same reexposure time was used for recent and remote memory extinction; therefore, more pronounced extinction was observed in wildtype animals in recent (Fig. 13C) than in remote (Fig. 13F) fear memory assessment. In contrast, in NEX null mutants the recent fear memory trace (Fig. 13C,D) was more stable than remote (Fig. 13F).

The relative stability of recent contextual memory in NEX mutants can be explained by two mechanisms. First, in addition to a defect in spatial memory acquisition, NEX mutants might also possess a deficit in those molecular mechanisms that are involved in memory reconsolidation and extinction in the hippocampus. Molecular profiling of the mutant hippocampus revealed a downregulation of fos in mice housed in regular cages (Tab. 3) and upregulation of jun upon prolonged exposure to the enriched environment (Tab. 5). Fos is known to be required for contextual fear conditioning and fear memory retrieval (Fleischmann et al., 2003; Maviel et al., 2004). Expression levels of immediate early genes c-fos and JunB, but not cjun, have been shown to increase in the CA1 region after memory retrieval (Strekalova et al., 2003). Since transcriptional profiling so far only comprised the CA3 region, the obtained expression data do not provide the whole spectrum of NEX targets in the hippocampus. Molecular profiling of the CA1 region of NEX null mutants is ongoing and will reveal whether expression of immediate early genes in other hippocampal regions correlates with the function of NEX in memory processes. 
In the course of recent memory extinction, the level of freezing response in wildtype animals is reduced significantly after 8 days of re-exposure (Fig. 13D), however, it failed to fall below that exhibited by NEX mutants. It is possible that a $20 \%$ freezing level (Fig. 13C) is the minimal possible representation of a memory trace that is resistant to extinction. Indeed, fear conditioning produces a very strong association between unconditioned and conditioned stimulus, which often lasts for a life time, and spontaneous recovery of fear response even after extinction is not unusual (Crawley, 2000; Suzuki et al., 2004; Frankland and Bontempi, 2005; Isiegas et al., 2006). Moreover, the course of extinction can not be easily predicted in advance for any given paradigm, since the strength of a memory trace depends on various parameters, such as shock intensity and age of the animal. The conditioning parameters used in this study have not been applied for extinction previously; therefore, a $20 \%$ freezing level might represent the minimal memory trace that will persist throughout the animal's life.

The rapid extinction displayed by NEX null mutants during the assessment of remote memory demonstrated lower stability of remote memory in the mutants compared to wildtype animals. This might be caused by weaker reconsolidation or reinforced extinction or both. Although recent memory recall mediates activation and transcriptional changes in the hippocampus, remote memory reconsolidation and extinction seem to be hippocampusindependent (von Hertzen and Giese, 2005). Imaging and inactivation studies have demonstrated that different regions of prefrontal cortex (PFC) play a crucial role in the recall of remote memory (Bontempi et al., 1999; Takehara et al., 2003; Maviel et al., 2004; Frankland et al., 2004). Both recent and remote memory extinction seem to be mediated by the prefrontal cortex (Frankland and Bontempi, 2005). Recent experimental data suggest that the prefrontal cortex might be able to send inhibitory signals to the hippocampus upon recall of remote spatial and contextual memories (Takehara et al., 2003; Maviel et al., 2004; Frankland et al., 2004), preventing the hippocampus from re-encoding existing memories. Therefore, a deficit in prefrontal cortex function or a failure to establish normal PFC-hippocampal connections might be responsible for the increased rate of remote memory extinction, observed in NEX null mutants. Interestingly, anterior cingulate cortex, a part of PFC, which is implicated in attention and cognitive control (Sotres-Bayon et al., 2004), receives a direct excitatory input from hippocampus and subiculum (Floresco et al., 1997). Delay or deficit of this input might account for the learning lag that NEX null mutants exhibit in the Morris water maze spatial learning task. Detailed investigation of NEX expression in the adult prefrontal cortex and PFC-hippocampal connections in NEX null mutants will help to better understand the particular role that NEX plays in remote memory formation and stability. 


\subsection{Outlook: part I}

The specific learning deficits exhibited by NEX null mutants in spatial and contextual learning tasks imply a disruption of proper hippocampal function or cortico-hippocampal connections in these animals; however, cellular and molecular mechanisms of this disruption are still unclear. Detailed studies of immediate early gene expression in the hippocampus and cortex of NEX null mutants will provide deeper insights into NEX-dependent processes of memory formation. The mild nature of the reported spatial learning phenotype suggests investigating changes of expression patterns in those null mutants that have been subjected to behaviour and memory tests. Molecular profiling of the CA1 region of the hippocampus will also shed light on the expression changes of particular genes that are involved in spatial learning.

It was recently observed that CREB, a key player of memory acquisition, controls NEX expression (Uittenbogaard et al., 2007). Together with the behavioural deficits of NEX null mutants, this suggests a role for NEX in the control of the molecular cascade that mediates neuronal plasticity and learning. Expression of some members of this cascade (fos, jun, prkar2b) in the CA3 region of the hippocampus is affected in NEX null mutants. Transcriptional analysis of other genes implicated in memory formation, such as zif268 and CaMKII, in the neocortex and CA1 hippocampal region of mutant mice will clarify whether these proteins act upstream or downstream of NEX. The presence of potential PKC and CaMKII phosphorylation sites in the transcriptional activation domain of NEX suggests a phosphorylation-dependent modulation of NEX activity. The exact mechanism of NEX phosphorylation and its effect on NEX-mediated transcription can be addressed in vitro in cell lines as well as in primary neuronal cultures.

Finally, the GFP-NEX transgenic mouse line, which is described in the following chapter, is a potential tool for the investigation of NEX function in behaviour and memory in a gain-offunction approach. Transgenic rescue of the learning phenotype of NEX-deficient mice could be performed by breeding the GFP-NEX line to NEX-Cre mutants (Goebbels et al., 2006).

\section{The effect of NEX overexpression on telencephalon development}

The lack of an obvious developmental phenotype in NEX null mutants might be explained by functional redundancy among NeuroD family members. NEX, NeuroD and NDRF have overlapping expression domains in the developing mouse telencephalon (Schwab et al., 1998). All three factors are expressed in pyramidal neurons of the embryonic neocortex; however, no deficit of pyramidal neuron development has been observed in single gene mutants (Bartholoma and Nave, 1994; Kume et al., 1998; Schwab et al., 1998; Chae et al., 2004; Cho 
and Tsai, 2004). In contrast, double mutants of NEX and NDRF exhibit defects in the formation of major cortical projections, and inactivation of all three factors causes a complete loss of pyramidal neurons in the hippocampus and gross abnormalities of cortical layer formation (Bormuth et al., in preparation). These observations confirm the redundancy hypothesis, but also indicate that further studies are necessary to fully understand individual functions of NeuroD family members in cortical development.

Studies of mouse mutants provide valuable information about the loss-of-function of the gene of interest; however, in case of functional redundancy particular phenotypic alterations are masked by the compensatory action of factors related to the gene of interest. Complementary overexpression studies often prove to be more useful in revealing the specific role of such genes. Therefore, a gain-of-function approach was used in this study to investigate the role of NEX in mouse brain development. The inducible nature of transgenic NEX overexpression in GFP-NEX mice allows using various Cre-recombinase harbouring lines to investigate NEX function in different developmental processes. In the next section Emx1-Cre mediated NEX overexpression in cortical neural progenitors will be discussed.

\subsection{Possible involvement of NEX in cell cycle control}

The neuronal differentiation factors NEX, NeuroD and NDRF act downstream from neuronal determination factors, such as Pax6 and neurogenins (Lee et al., 1995; Sommer et al., 1996; Farah et al., 2000; Hevner et al., 2006), and their expression is mostly restricted to committed cells of the mouse telencephalon. Thus, NeuroD is detected in the upper subventricular zone and intermediate zone (Lee et al., 2000), and the first NEX-positive cells are located in the preplate (Goebbels et al., 2006). Moreover, NEX expression in the subventricular zone seems to mark those cells that are already committed to a glutamatergic neuronal fate $(\mathrm{Wu}$ et al., 2005). The absence of these factors from non-committed progenitor cells in the ventricular zone suggests a functional interplay between cell cycle exit, neuronal migration and differentiation in the developing cortex. Several in vitro studies have demonstrated the existence of a mutual inhibition between NeuroD and genes involved in cell cycle progression (Mutoh et al., 1998; Ratineau et al., 2002; Deisseroth et al., 2004). NEX has been shown to enhance expression of cyclin-dependent kinase inhibitors, thus promoting cell cycle arrest in PC12 cells (Uittenbogaard and Chiaramello, 2004). These results indicate a functional relationship between cell cycle and neuronal differentiation control; however, the question of whether these mechanisms also operate in vivo has not been addressed.

Transcriptional profiling performed in this study demonstrated that several genes involved in cell growth and cell cycle control are differentially regulated in NEX mutants 
compared to wildtype (Tab. 3-5). Interestingly, cdkn1a, nedd9 and reelin are upregulated in null mutants upon prolonged environmental enrichment (Tab. 5; Fig. 6E-H). This indicates that neurons of the CA3 region acquire a state of certain immaturity in the absence of NEX. This is a first in vivo observation that suggests an involvement of NEX in the repression of neural precursor cell markers and regulators of cell cycle progression. Since common molecular mechanisms of differentiation operate in embryonic and adult neurogenesis (Hevner et al., 2006), it is conceivable that NEX regulates the expression of cell cycle-related genes in the developing and adult neocortex.

To test the hypothesis that NEX is involved in cell cycle control, a gain-of-function experiment was performed, using the GFP-NEX transgenic mouse line, in which overexpression of NEX was induced in cortical progenitors by Emx1 promoter-driven Cre-recombinase (Gorski et al., 2002).

\subsection{Cortical development is impaired in GFP-NEX*Emx1-Cre animals}

Transgenic mice harbouring the GFP-NEX transgenic construct exhibited uniform green fluorescence (Fig. 15A). When this line was crossed to the Emx1-Cre mouse line (Gorski et al., 2002), the reduction of fluorescence in the cortex of double transgenic animals demonstrated the efficient excision of eGFP in the brain of GFP-NEX*Emx1-Cre mice. Examination of green fluorescence in primary neuronal cultures showed that while the majority of neurons were GFPpositive in GFP-NEX cultures, only few cells in GFP-NEX*Emx1-Cre exhibited green fluorescence (Fig. 15E,F). Importantly, the total number of cells in the culture was similar for both control and mutant cultures, demonstrating that overexpression of NEX does not cause neuronal death.

Unfortunately, transgenic expression of recombinant NEX protein in transgenic animals could not yet be confirmed directly. All attempts to detect the AU1 tag by immunocytochemistry on primary cultures and immunohistochemistry on brain sections failed (data not shown). Although successful $\alpha$-AU1 immunohistochemistry on brain sections has been reported previously (Shevtsova et al., 2006), it is possible that in this study the AU1 tag was subjected to posttranslational modifications or simply masked by the protein structure and therefore could not be accessed by the antibody. A recently published report of spontaneous HA-tag cleavage from a recombinant protein in cultured cells (Schembri et al., 2007) demonstrates that modifications of tagged proteins are unpredictable and can even result in a complete loss of immunoreactivity. The lack of a functional antibody directed against NEX itself further reduced the chances of detecting the recombinant protein in primary cell culture or on brain sections. However, results obtained from preliminary cell culture experiments that clearly demonstrated eGFP excision and 
expression of AU1-tagged NEX in vitro (Fig. 14), together with the observation that fluorescence is reduced in cortical neurons of transgenic mice (Fig. 15E,F), indicate expression of transgene-derived NEX in vivo. Ongoing semi-quantitative in situ hybridization, Northern blotting and QRT-PCR will likely provide a direct proof of transgene expression in the GFPNEX line.

Examination of brains from GFP-NEX*Emx1-Cre mice revealed a severe size reduction of cerebral hemispheres (Fig. 16A). Reduction of brain size was observed in both newborn and adult animals (Fig. 16B,C). GFP-NEX animals were additionally bred to the NEX-Cre mouse line (Goebbels et al., 2006), in which Cre expression is almost completely restricted to postmitotic neurons. Examination of brains from GFP-NEX*NEX-Cre double transgenic animals demonstrated no differences in brain size (Fig. 16D). These data suggest that hemisphere size reduction in GFP-NEX*Emx1-Cre mice represents a genuine effect of Emx1-Cre-mediated NEX overexpression and is not due to general toxicity of NEX transgene.

To analyse in more detail the reason for the reduced cerebral hemisphere size, histological analysis was performed. $\alpha-\mathrm{NeuN}$ staining of P0 and adult brain sections revealed a reduction in cortical thickness in NEX gain-of-function mutants (Fig. 17). A comparison of the architecture of the somatosensory cortex demonstrated that the density of neuronal nuclei in the cortex and hippocampus of mutant mice appeared comparable to that in wildtype animals (Fig. 17, 18). All cortical layers were present in the mutant, however, the thickness of all layers was reduced, and deeper layers (V and VI) were more affected than superficial layers (II-IV; Fig. 18). The fact that predominantly the formation of deep cortical layers was disturbed indicated that neocortical development was affected at early stages; therefore, detailed examination of telencephalon development at embryonic stages E12,5 and E13,5 was performed.

TUNEL staining for apoptotic cells was performed on paraffin sections of GFP-NEX and GFP-NEX*Emx1-Cre embryos. Whereas virtually no TUNEL-positive cells were detected in the developing telencephalon of GFP-NEX single transgenic animals at E12,5 or 13,5, the GFPNEX*Emx1-Cre double transgenic mutants showed massive apoptosis in the ventricular zone at both stages (Fig. 19). Immunostaining against neuronal $\beta$-TubulinIII (Tuj1) revealed that the density of differentiating neurons in the forming cortical plate at E13,5 was comparable in mutants and control animals. In contrast, in GFP-NEX*Emx1-Cre mice Tuj1-positive cells were also detected within the ventricular zone, indicating premature neuronal differentiation or a migration defect or both. Whether ectopic expression of Tuj1 in the ventricular zone of the mutants led to programmed cell death of the progenitors, remains an open question, since double labelling for Tuj1 and TUNEL has not yet been performed. 
Recently a study of Pax6 overexpression in cortical progenitors was reported (Berger et al., 2007). The authors used a similar Cre-recombinase mediated overexpression system to drive expression of different Pax6 isoforms. They observed cortical size reduction and progenitor cell death upon overexpression of Pax6, but not Pax6-5a, in cells of the Emx1 lineage. Cell cycle dysregulation in early cortical progenitors and ectopic expression of differentiation markers in the ventricular zone was also detected (Berger et al., 2007). Interestingly, the authors mention an upregulation of NEX mRNA upon Pax6 overexpression in early development. These data suggest that Pax6 and NEX are involved in the same cascade of cell cycle regulation during cortical development. The effects appear specific to progenitor cells of the Emx1 lineage at early stages of cortical development (Berger et al., 2007 and this study). In contrast, Pax6 or NEX overexpression in differentiating neurons, mediated by breeding both transgenic lines to NEXCre mice (Goebbels et al., 2006), did not seem to alter cerebral hemisphere size or cortical development (Berger et al., 2007, see also Fig. 16D).

Pax6 is an evolutionary conserved transcription factor that plays an important role in brain development, in particular in cortical patterning. Pax6 expression starts in the neuroepithelium as early as E8,5 and stays high in the ventricular zone until E15,5 (Stoykova et al., 2000). Different levels of Pax6 expression along the anterior-posterior axis play a role in progenitor regionalization (Bishop et al., 2000). Analysis of the regulatory elements of the murine Nex gene revealed the presence of Pax-responsive DNA elements in the NEX promoter (Uittenbogaard et al., 2007). Taken together, these data are compatible with the conclusion that NEX acts downstream of Pax6 in promoting cell cycle exit, migration and differentiation of cortical progenitors.

\subsection{Outlook: part II}

The production of the GFP-NEX mouse line and NEX overexpression in cortical progenitors is a first step towards a better understanding of the role of NEX in telencephalon development. Detailed studies of progenitor cell cycle progression (e.g. by BrdU labelling), their adhesive properties and neuronal marker expression pattern will shed light on the molecular mechanism mediating developmental defects in GFP-NEX*Emx1-Cre mutants.

Although Emx1-Cre-mediated overexpression of NEX provides important information on the role of NEX in cell cycle and migration control, the ectopic expression of a differentiation gene in mitotically active progenitors is to a certain extent artificial and does not reveal the natural function of NEX in telencephalon development. The ability of NEX to induce cytoskeletal and synaptic protein expression in vitro (Uittenbogaard and Chiaramello, 2004) and the failure of axonal outgrowth in mice lacking NEX and NDRF (Bormuth et al., in preparation), 
suggest an important role for NEX in neuronal development and maintenance. Investigation of NEX overexpression in postmitotic neurons in GFP-NEX*NEX-Cre double transgenic animals will therefore continue to decipher the in vivo function of NEX during pyramidal cell differentiation. The behavioural phenotype of NEX null mutants, described in the first part of this thesis, implies an involvement of NEX in cognitive functions, such as learning and memory. The GFP-NEX mouse line could be used to assess potential dosage effect of NEX on adult neuronal plasticity. The NEX-Cre mouse line harbours a NEX null allele, and breeding to GFPNEX mice provides the possibility to achieve a graded series of NEX expression levels. However, in the case of NEX-Cre-mediated NEX overexpression adult brain function can not be completely separated from a possible developmental effect on neuronal morphology. Therefore, the CaMKII-Cre transgenic mouse line (Minichiello et al., 1999) with postnatal onset of Cre expression could be used to overexpress NEX in adult neurons, and the effect could be assessed in behavioural experiments. 


\section{Generation and analysis of NEX-htTA transgenic mice}

Homologous recombination in bacteria (reviewed in Copeland et al., 2001; Hatten and Heintz, 2005) was used to place a humanized version of the tetracycline transactivator (htTA; Kim, 2001) under control of regulatory sequences of the Nex gene, located on a $200 \mathrm{~kb}$ bacterial artificial chromosome (BAC). The Nex locus was chosen to drive htTA expression in postmitotic pyramidal neurons throughout brain development and in adulthood (Bartholomä and Nave, 1994; Shimizu et al., 1995; Schwab et al., 1998). It has previously been shown as well suited to direct expression of heterologous genes, such as Cre recombinase, to postmitotic pyramidal neurons (Goebbels et al., 2006). The Tet-off system has been successfully applied to the analysis of the brain (Mayford et al., 1996; Mack et al., 2001; Yasuda and Mayford., 2006). Two reporter mouse lines, GFPG3 (harbouring eGFP and $\beta$-galactosidase cassettes under the control of tetO promoter; Krestel et al., 2001) and tetO-Cre (Schonig et al., 2002), were used to investigate NEX-htTA driven gene expression in development and postnatal brain.

\subsection{NEX-htTA transgene-mediated reporter gene expression}

Whole-mount X-gal staining of NEX-htTA*GFPG3 transgenic embryos showed NEXlike reporter expression pattern during early embryonic development, as $\beta$-galactosidase activity was detected in the developing telencephalon starting from E12 and was localized to cerebral hemispheres, cerebellar anlage and spinal cord (Fig. 21). No X-gal staining was detected in single transgenic NEX-htTA embryos or in double transgenic NEX-htTA*GFPG3 embryos, that have received doxycycline through their mother (Fig. 21B,C). Therefore, in early phases of cortical development htTA expression appears to faithfully recapitulate endogenous NEX expression. X-gal staining of NEX-htTA*GFPG3 double transgenic mice at postnatal stages revealed serious reduction of $\beta$-galactosidase activity in the cortex. Already at P5 reporter activity was restricted to amygdala and hippocampus, with only very few X-gal-positive cells present in the neocortex (Fig. 24A).

The lineage analysis of NEX-htTA-mediated recombination, performed on NEXhtTA*tetO-Cre*Rosa26 triple transgenic animals at P24, revealed Cre-mediated recombination in hippocampus, piriform and entorhinal cortex, supramamillary bodies and amygdala (Fig. 22A). Doxycycline treated animals showed no X-gal staining in the brain (Fig. 22B). Thus, htTA activity in NEX-htTA transgenic line was efficiently inhibited by doxycycline administration both in the embryonic development and in the adult.

Interestingly, X-gal staining in the adult cortex was restricted to deeper cortical layers. Co-staining for NeuN demonstrated virtually no $\beta$-galactosidase activity in layers II and III, 
whereas many cells in layers VI and V and some cells in layer VI were X-gal-positive (Fig. 23). Thus, the cortical expression pattern of htTA did not fully recapitulate the pattern of NEX lineage, as observed in the NEX-Cre mouse line, where Cre-mediated recombination was detected in all cortical layers (Goebbels et al., 2006). Such a restriction of cortical htTA expression to deeper layers indicates a deficit in the regulatory elements of the Nex gene in this transgenic line.

Further analysis of NEX-htTA transgene activity in postnatal brain was performed on NEX-htTA*tetO-Cre*Rosa26 triple transgenic animals. Here, doxycycline treatment was performed to suppress Cre activation during embryonic development: the pregnant mothers were given drinking water containing $100 \mathrm{mg} / \mathrm{l}$ of doxycycline, which was replaced with regular water after birth. Pups that received doxycycline through the mother till birth were analysed for reporter expression at 6 and 8 weeks of age and revealed a great reduction of $\beta$-galactosidase activity in the cerebral cortex (Fig. 24B-D). An overview of $\beta$-galactosidase expression in different reporter lines is given in Tab. 6. Similar patterns of reporter expression were observed in the adult brain of NEX-htTA*GFPG3 double transgenic animals that received no doxycycline and NEX-htTA* tetO-Cre*Rosa26 triple transgenic animals, in which htTA activity was suppressed by doxycycline till birth. Together these data suggest that although high htTA activity is observed in the developing telencephalon, postnatally prominent htTA expression is restricted to the hippocampal formation and a small population of cells in entorhinal cortex (Fig. $24 \mathrm{C}, \mathrm{D})$. The reason for the restriction of postnatal expression to only a subset of brain areas remains to be elucidated.

Several reasons can account for the loss of transgene activity in certain cell populations. Although the effect of transgene integration site is considered to be minor in experiments using BAC-based constructs, it can not be fully overruled. The fact that the NEX-htTA BAC transgene was not linearized prior to oocyte injection can also account for the loss or incorrect arrangement of regulatory elements during integration into the genome. Finally, the presence of point mutations in the transgenic construct could cause a loss of superficial cortical layer-specific regulatory sequences in NEX-htTA line. A recent study of the mouse NDRF promoter has demonstrated that approximately a $10 \mathrm{~kb}$ DNA region, located upstream of the transcriptional start site of NDRF, is sufficient to recapitulate the endogenous NDRF expression pattern in a transgenic reporter approach (Lin et al., 2004). Because of conserved DNA sequences in their promoter region and very similar expression patterns, it is reasonable to assume that cue regulatory elements for mouse NEX expression are also located within $10 \mathrm{~kb}$ upstream of the NEX coding region. Therefore, it is possible that a break or a point mutation within this $10 \mathrm{~kb}$ 
region might account for the restricted expression of htTA observed in the NEX-htTA BAC transgenic line.

\subsection{Outlook: part III}

The NEX-htTA mouse line provides a novel tool for studying mechanisms of neocortical development and neuronal plasticity. The inducible nature of the Tet-off system allows for the temporal modulation of expression of a gene of interest. It can be used for loss-of-function (e.g. RNAi or expression of a dominant-negative variant; Hong et al., 2007), as well as gain-offunction approaches. The combination of a tTA line with the tetO-Cre mouse line allows for inducible, but permanent recombination on genomic DNA level and is suitable for the conditional inactivation or overexpression of genes of interest.

The NEX-htTA transgenic line is currently being used together with the tetO-Cre line and an inducible diphtheria toxin line (Brockschneider et al., 2004) in collaboration with Anjana Nityanandam and Dr. Victor Tarabykin (Max-Planck-Institute of Experimental Medicine) to investigate mechanisms of cortical development. One intriguing question of brain development is whether dividing progenitors in the ventricular zone receive a feedback signal from the cortical plate and whether the production of layer-specific neuronal cell types depends on such a feedback. The restricted expression of htTA to layers V and VI in NEX-htTA mice together with the diphtheria toxin mouse line will allow to selectively kill neurons that would normally populate layers V and VI. Such an experiment will address the question as to whether a dialogue between ventricular zone and the forming cortical plate exists. Moreover, it will reveal whether the formation of specific layers depends only on intrinsic properties of the progenitors or can be modulated by extrinsic signals.

The unique postnatal expression pattern of NEX-htTA also provides a possibility to study adult neuronal plasticity in hippocampus and entorhinal cortex. These brain areas are not only involved in hippocampal learning, but also play an important role in hippocampo-cortical connections (Yasuda and Mayford, 2006). Inducible gene inactivation or transgene expression in these areas will prove useful for detailed studies of behaviour and memory formation. 


\section{MATERIALS}

\section{Chemicals and laboratory supplies}

\subsection{Laboratory equipment}

\section{Computer hardware}

IBM PC Intel ${ }^{\circledR}$ Pentium ${ }^{\circledR} 4$ CPU

HP Colour Laser Jet

OKI C9200

Computer Point, Goettingen

Hewlett Packard, Boeblingen

OKI Systems, Duesseldorf

\section{Computer software}

Acrobat Reader 7.0

Adobe Photoshop CS2

Illustrator 10.0

DNA Star 6.0

Endnote 9.0

Firefox web browser 1.5.0.7

Microarray suite 4.0

Microsoft Office X

Openlab 3.1.7

pDRAW 32.1.1.91

Roche: Universal Probe Library Assay

Design Center

Image J 1.34s

Moti (Mobilitäts-Mess System) 4.16

TSE Rota-Rod V2.6

TSE Fear Conditioning System

VideoMot2

Adobe, San Jose, CA

DNA Star Inc., Madison, WI

Thomson ResearchSoft, Carlsbad, CA

Mozilla Foundation, Mountain View, CA

Affymetrix, UK

Microsoft, Redmond, WA

Improvision, Lexington, MA

www.acaclone.com

www.roche-applied-science.com

rsb.info.nih.gov/ij/

Hasomed GmbH, Magdeburg

Technical+Scientific Equipment (TSE)

$\mathrm{GmbH}$

\section{Equipment}

Agilent 2100 Bioanalyser

Axiophot microscope

Camera CCTV (Panasonic)

Cryostate Jung CM 300

Digital camera ProgRes C14

GeneChip Fluidics Station 400

GenePulser Xcell

HP GeneArray Scanner

Kappa DX20 H-FW digital camera

Leica DM IRBE (inverted microscope)

Leica DM RXA

MICROM AP 280

MICROM HMP 110

Microtome HM 400

Philips DVDR 3330H

Agilent Biotechnologies, Boeblingen

Zeiss, Goettingen

TSE GmbH, Bad Homburg

Leica, Solms

Jenoptik, Jena

Affymetrix, UK

Biorad, Muenchen

Affymetrix, UK

Kappa, Gleichen

Leica, Solms

Stemi SV6 binocular

Microm, Walldorf

Philips, Goettingen

Zeiss, Goettingen 
SDS-PAGE and Western Blot chamber XCell II

Veritas Microdissection System

X-ray film developer KODAK X OMAT

7500 Fast Real-time PCR System

UV-Illuminator

\subsection{Laboratory supplies}

AquaPolyMount

CapSure Macro LCM Caps

Cryo-vials

Tissue Kit

MinElute Gel Extraction Kit

Plasmid Mini Kit

Plasmid Midi Kit

Plasmid Endo-Free Maxi Kit

RNAlater, RNA stabilization reagent

RNeasy mini kit

rNTPs

Zeocin

ECL-Hyperfilms

PVDF Membrane Hybond P

Glass slides and coverslips

Cell culture dishes and plates

96-well plates for RT-PCR

pGEM-T Vector System

pGEM-Teasy Vector System

ECL Plus Western-Blot Detection

Reagents

LSAB2®System-HRP

Liquid DAB + Substrate

PEN membrane frame slides

\subsection{Enzymes}

Pfu Taq Polymerase

HotStart MasterMix

Alkaline phosphatase, CIP

Restriction emzymes

RNaseH

RNAsin

RQ1 DNase, Rnase free

Sp6, T3, T7 RNA polymerases

T4 DNA ligase

SuperScript III
Invitrogen, Karlsruhe

Molecular devices, USA

Kodak, Stuttgart

Applied Biosystems, Warrington

Intas UV-Systeme, Heidelberg

Polysciences Inc, Warrington, PA

Molecular Devices, USA

Nalgene, Rochester, NY

Invitek, Berlin

Qiagen, Duesseldorf

Ambion, Darmstadt

Qiagen, Duesseldorf

Promega, Mannheim

Invitrogen, Karlsruhe

Amersham Biosciences, Freiburg

Menzel, Marienfeld

BD Falcon, Hamburg

Applied Biosystems, Warrington

Amersham Biosciences, Freiburg

Dako North America, Carpinteria, CA

Leica, Solms

Stratagene, La Jolla, CA

Qiagen, Duesseldorf

New England Biolabs, Frankfurt

Promega, Mannheim

Invitrogen, Karlsruhe 


\subsection{Antibodies}

Rat $\alpha$-BrdU monoclonal

Mouse $\alpha$-TUJ1 monoclonal

Mouse $\alpha$-NeuN monoclonal

Mouse $\alpha$-AU1 monoclonal

Mouse $\alpha$-GFP monoclonal

HRP-conjugated goat $\alpha$-mouse

HRP-conjugated goat $\alpha$-rabbit

Cy3-conjugated goat $\alpha$-mouse

Cy3-conjugated goat $\alpha$-rabbit

Alexa-488-conjugated goat $\alpha$-rat

AP-coupled $\alpha$-DIG Fab fragments

\subsection{Mouse lines}

KNEX ( Schwab et al, 1998)

GFPG3 (Krestel et al., 2001)

Rosa26 (Soriano et al., 1999)

tetO-Cre (Schonig et al., 2002)

Emx1-Cre (Gorski et al., 2002)

NEX-Cre (Goebbels et al., 2006)

\author{
Abcam, UK \\ Covance, UK \\ Chemicon, USA \\ Covance, UK \\ Boehringer Mannheim \\ Dianova, Hamburg \\ Dianova, Hamburg \\ Dianova, Hamburg \\ Dianova, Hamburg \\ Molecular Probes, Karlsruhe \\ Boehringer, Mannheim
}

Dr. Markus Schwab, MPI of Experimental Medicine, Goettingen Dr. Rolf Sprengel, MPI of Medical Research, Heidelberg

Dr. Victor Tarabykin, MPI of Experimental Medicine, Goettingen

Dr. Sandra Goebbels, MPI of Experimental Medicine, Goettingen

\subsection{Cell lines}

$\operatorname{COS} 1$

PC12

Tumour cells from the kidney of African green monkey

Rat adrenal pheochromocytoma cells

\subsection{Bacterial strains}

E.coli XL-1 blue

E.coli DH5 $\alpha$

E.coli DH10B

EL250, EL350
Stratagene, La Jolla, CA

Invitrogen, Karlsruhe

Dr. N. Copeland, NCI Frederick, MD

\subsection{Oligonucleotides}

\author{
Name \\ Genotyping KNEX \\ KNEX fwd \\ KNEX rev \\ Genotyping BEAN \\ BEAN fwd \\ BEAB rev \\ Genotyping NEX-Cre \\ Cre rev \\ NEX rev \\ NEX fwd \\ Genotyping Emx-Cre \\ EXEC rev
}

Sequence

In-house Nr.

CAAGTTGTCCTTCGAGGAAAGAGC

GATACAGACAAGAGGGAAGGG

2071

CCTGTAGGTGTCCATGGT

GGACGAGCTGTACAAGTA

9543

9544

CCG CAT AAC CAG TGA AAC AG

GAG TCC TGG AAT CAG TCT TTT TC

3131

CCAGGCTAAGTGCCTTCTCTACA 
Name

EXEC fwd

Genotyping Rosa 26

Rosa 1

Rosa 2

Rosa 3

Genotyping GFPG3

GFPG3 fwd

GFPG3 rev

Genotyping TAXB

TAXB fwd

TAXB rev

Genotyping tetO-Cre

Cre fwd

Cre rev

Introducing AU1 tag

AU1-NEX rev

AU1-NEX fwd

Mutagenesis

NexS230A rev

NexS230A fwd

Primer in NEX Exon1

NEXexon 1 fwd

Homology arms

NEX-htTA rev/inner

NEX-htTA rev/outer

NEX-htTA fwd/inner

NEX-htTA fwd/outer

Real time PCR

$\beta$-actin fwd

$\beta$-actin rev

18sRNA fwd

18sRNA rev

TTR fwd

TTR rev

Prkar2b fwd

Prkar2b rev

Npas4 fwd

Npas4 rev

Nedd9 fwd

Nedd9 rev

Reelin fwd

Reelin rev

Cdkn1a fwd

Cdknla rev
Sequence

AATGCTTCTGTCCGTTTGCCGGT

In-house Nr.

5383

AAA GTC GCT CTG AGT TGT TAT 3735

GCG AAG AGT TTG TCC TCA ACC $\mathbf{3 7 3 6}$

GGA GCG GGA GAA ATG GAT ATG $\quad 3737$

GCGGAGAGGGTGAAGGTGATGC $\quad \mathbf{2 0 0 8}$

CAGGGCCGTCGCCGATTGG

2009

CTG CTC TTG GAG TCT TAA CG

5791

5792

CCAGGCTAAGTGCCTTCTCTACA $\mathbf{5 3 8 2}$

AATGCTTCTGTCCGTTTGCCGGT $\mathbf{5 3 8 3}$

CGGATCCGCGGCCGCTTAATTATGAAAAA $\mathbf{8 2 4 4}$

CTGCATTTAATTCATCTTGCAT

GAATTCGCCACCATGGACACCTACAGGTA

CAT CTTAACACTACCGTTTGAC

GTAATTGTAGGGTTTCATGGCCTTGGAAT

TATCAAGAGT

ACTCTTGATAATTCCAAGGCCATGAAACC

CTACAATTAC

GATGCGACACTCAGCCTGAA

9762

TGG GAG GTG AAT GAC CAC TGT TTA TT T TCA

4612

TTT TCC TCA AGT GCC AAG CTA CTC GCG AC

CGC AGC CCA CAA GCA TCT GCT TTG CC T TAA

4613

TTA GAG TGG GAG GTG AAT GAC CAC TGT

GCT GGC ATT TTT TTT CAG GTA AAT T AA GGT

TAA AGA ACC ATG TCC AGA C TG GAC AAG AGC

TAT CAA TGC TAA AAA TGA AAA CAC TTT ACA

TAC TGT GCT GGC ATT TTT TTT CAG GT

CTTCCTCCCTGGAGAAGAGC

ATGCCACAGGATTCCATACC

AAATCAGTTATGGTTCCTTTGGTC

GCTCTAGAATTACCACAGTTATCCAA

GACCATCAGAGGACATTTGGA

TCGCTGGACTGGTATTTGTG

GTGCTCTGTGGGGTTTG

TTCCTCTTTTTGGCATTGTTTT

AGGGTTTGCTGATGAGTTGC

TTCCCCTCCACTTCCATCT

GCGGAGTTGGATGGATGAT

CTCCTTCTGCTGTCGCTCA

TCACCATTGCCATTCCAAG

CTTGGATCCATCGGAATCTG

TCCACAGCGATATCCAGACA

GGACATCACCAGGATTGGAC
4614

4615

9146

9147

9145

9166

9874

9875

12446

12447

12448

12449

12450

12451

12452

12453

12454

12455 


\subsection{Vectors}

\subsubsection{Cloning and expression vectors}

Name
pcDNA3
pRK5
pBluescript KS
pGEM
pGEMeasy
pEGFP C1
pDsRed Express
p $\beta$-actin
pTetO-lacz-GFP
pTOBiTRUE
pRL-CMV
pRL-SV-40
pRL-TK
pGL2

\section{Promoter \\ CMV \\ CMV}

T3 and T7

T7 and Sp6

T7 and Sp6

CMV

CMV

CMV-ßactin

tetO

tetO

CMV

SV-40

TK

TATA
Antibiotic resistance

AmpR

AmpR

AmpR

AmpR

AmpR

KanR

KanR

AmpR

AmpR

AmpR

AmpR

AmpR

AmpR

AmpR

\subsubsection{Bacterial artificial chromosomes}

RP23-268011

BAC PAC resources, Oakland, CA

\section{Media, buffers and solutions}

All media for mammalian cell line culture were based on DMEM Dulbecco's basic medium (1g/1 glucose, F12), purchased from Invitrogen, Karlsruhe. Heat inactivated foetal calf serum (FCS) and/or horse serum, $200 \mathrm{mM}$ glutamax (Invitrogen) and 1\% penicillin/streptavidin were added prior to use.

\begin{tabular}{lccc}
\multicolumn{1}{c}{ Cell line } & Basic medium & FCS content & HS content \\
COS 1 & DMEM & $10 \%$ & - \\
PC12 & DMEM & $15 \%$ & $5 \%$
\end{tabular}

Medium for cryoconservation contained additionally 20\% dimethylsulfoxide (DMSO).

Neuronal primary cell culture medium

Neurobasal medium (Gibco)

B27 Supplement (Gibco) 1\%

Glutamax (Gibco) 1\%

Penicillin/Streptomycin (Sigma) 1\%

MK801 (Tocris, $10 \mathrm{mM}$ Stock) 1:1000

NGF (Promega, $50 \mathrm{ng} / \mu \mathrm{l}$ Stock) 1:200

FGF (PeproTech, 10 ng/ $\mu$ l Stock) 1:100
$100 \mathrm{ml}$

$1 \mathrm{ml}$

$1 \mathrm{ml}$

$1 \mathrm{ml}$

$100 \mu 1$

$200 \mu \mathrm{l}$

$1000 \mu \mathrm{l}$ 
Transfection medium

OptiMEM ${ }^{\circledR}$ - Medium (Gibco BRL, Karlsruhe)

Trypsin-EDTA (10X)

Trypsin

EDTA

PBS (phosphate-buffered saline) 10x

$\mathrm{NaCl}$

$\mathrm{Na}_{2} \mathrm{HPO}_{4}$

$\mathrm{NaH}_{2} \mathrm{PO}_{4}$

\section{Cell electroporation buffer}

$\mathrm{KH}_{2} \mathrm{PO}_{4}$

$50 \mathrm{mM}$

$\mathrm{CH}_{3} \mathrm{COOK}$

$\mathrm{KOH}$

Adjust $\mathrm{pH}$ to 7,4

$\mathrm{MgCl}_{2}$

\section{LB-Medium (Luria's Broth)}

Yeast extract

Bacto-Pepton $\mathrm{pH}$ 7,5

$\mathrm{NaCl}$

For plates, add agar
$1,3 \mathrm{M}$

$70 \mathrm{mM}$

$30 \mathrm{mM}$
$5 \mathrm{~g}$

$6,84 \mathrm{mM}$

Autoclave before use; use still warm agar-containing medium to pour the plates.

LB/Amp: 0,1 mg/ml Ampicillin in LB-Medium

LB/Kan: $0,05 \mathrm{mg} / \mathrm{ml}$ Kanamycin in LB-Medium

$\mathrm{LB} / \mathrm{Amp} / \mathrm{IPTG}$ :

$35 \mu \mathrm{g} / \mathrm{ml}$ 5-Bromo-4-Chloro-3-Indolyl- $\beta$-D-Galactopyranosid (X-Gal);

$15 \mu \mathrm{g} / \mathrm{ml}$ Isopropyl- $\beta$-D-thiogalactopyranosid (IPTG);

$0,1 \mathrm{mg} / \mathrm{ml}$ Ampicillin in LB-Medium.

\section{TB (terrific broth) medium}

Trypton

Yeast extract

Glycerin

$\mathrm{H}_{2} \mathrm{O}$

Autoclave, let cool down to $60^{\circ} \mathrm{C}$.

Add $100 \mathrm{ml}$ phosphate buffer:

$\mathrm{KH}_{2} \mathrm{PO}_{4}$

$\mathrm{K}_{2} \mathrm{HPO}_{4}$

$$
\begin{aligned}
& 0,5 \%(\mathrm{w} / \mathrm{v}) \\
& 1 \%(\mathrm{w} / \mathrm{v}) \\
& 1 \%(\mathrm{w} / \mathrm{v}) \\
& 1,5 \%(\mathrm{w} / \mathrm{v})
\end{aligned}
$$


TB jap

PIPES

$10 \mathrm{mM}$

$\mathrm{CaCl}_{2}$

$15 \mathrm{mM}$

$\mathrm{KCl}$

$250 \mathrm{mM}$

Adjust pH to 6,7, then add:

$\mathrm{MnCl}_{2}$

$55 \mathrm{mM}$

Sterile filtrate.

DNA loading dye

Bromophenol blue

Xylene cyanol

$0.25 \%$

Ficoll (type 400)

$0.25 \%$

$15 \%$

$\mathrm{dH}_{2} \mathrm{O}$

to $10 \mathrm{ml}$; store at $-20^{\circ} \mathrm{C}$

\section{0x TAE}

Tris-base

Glacial acetic acid

EDTA (0,5 M, pH 8)

$242 \mathrm{~g}$

$57,1 \mathrm{ml}$

$100 \mathrm{ml}$

$\mathrm{dH}_{2} \mathrm{O}$

to $1000 \mathrm{ml}$

10x TBE

Tris-base

$108 \mathrm{~g}$

Boric acid

$55 \mathrm{~g}$

EDTA (0,5 M, pH 8)

$40 \mathrm{ml}$

$\mathrm{dH}_{2} \mathrm{O}$

to $1000 \mathrm{ml}$; filter solution

Injection buffer for transgene constructs

Tris $\mathrm{pH} 7,5$

$10 \mathrm{mM}$

EDTA pH 8,0

$0,1 \mathrm{mM}$

Lysis buffer (LyB)/Nuclear lysis buffer (NLyb)

Hepes pH 7,5

Glycerol

$\mathrm{NaCl}$

$\mathrm{MgCl}_{2}$

EDTA

Triton-X-100
$20 \mathrm{mM}$

$20 \%$

$10 \mathrm{mM}$ (500 mM for NLyB)

$1,5 \mathrm{mM}$

$0,2 \mathrm{mM}$

$0,1 \%$

Dissolve in $\mathrm{H}_{2} \mathrm{O}$; add fresh before use: $1 \mathrm{mM}$ DTT and 1 "Complete Mini" proteinase inhibitor tablet (Roche) per $10 \mathrm{ml}$. 


\section{RIPA Buffer}

$\begin{array}{ll}\text { Tris-HCl pH 7,4 } & 50 \mathrm{mM} \\ \mathrm{NaCl} & 150 \mathrm{mM} \\ \text { EDTA } & 1 \mathrm{mM} \\ \text { Triton-X-100 } & 1 \% \\ \text { Na-Deoxycholat } & 1 \% \\ \text { SDS } & 0,1 \%\end{array}$

Dissolve in $\mathrm{H}_{2} \mathrm{O}$; add 1 "Complete Mini" proteinase inhibitor tablet (Roche) per $10 \mathrm{ml}$ fresh before use.

MES electrophoresis buffer (10x)

MES

Tris-Base

SDS

EDTA
$1 \mathrm{M}$

$1 \mathrm{M}$

$2 \%$

$20 \mathrm{mM}$

Keep in dark place; $1 \mathrm{x}$ buffer $\mathrm{pH}=7,3$.

\section{LDS-loading buffer (4x)}

Tris-Base

Tris- $\mathrm{HCl}$

Lithiumdodecylsulfat (LDS)

EDTA

Glycerol

Serva Blue G250 (1\% solution)

Phenol Red (1\% solution)

Keep at $-20^{\circ} \mathrm{C}$; 1 xbuffer $\mathrm{pH}=8,5$.

\section{Transfer buffer for western blotting}

Bicine

Bis-Tris

EDTA

Chlorobutanol

Keep at $+4^{\circ} \mathrm{C} ; 1 \mathrm{x}$ buffer $\mathrm{pH}=7,2$.

TBST (wash buffer for Western blotting)

Tris-base, $\mathrm{pH} 7,5$

$\mathrm{NaCl}$

Tween 20

\section{Blocking buffer for Western blotting}

$5 \%$ non-fat dry milk in TBST
$564 \mathrm{mM}$

$424 \mathrm{mM}$

$8 \%(\mathrm{w} / \mathrm{v})$

$2,04 \mathrm{mM}$

$40 \%(\mathrm{w} / \mathrm{v})$

$7,5 \%(\mathrm{v} / \mathrm{v})$

$2,5 \%(\mathrm{v} / \mathrm{v})$
$500 \mathrm{mM}$

$500 \mathrm{mM}$

$20 \mathrm{mM}$

$1 \mathrm{mM}$
$50 \mathrm{mM}$

$150 \mathrm{mM}$

$0,03 \%(\mathrm{v} / \mathrm{v})$ 
Western blot membrane stripping buffer

Tris- $\mathrm{HCl} \mathrm{pH} 6,7$

$62,5 \mathrm{mM}$

SDS

$2 \%(\mathrm{w} / \mathrm{v})$

$\beta$-Mercaptoethanol

$100 \mathrm{mM}$

\section{Lysis buffer for luciferase assay}

Dilute 5x Passive lysis buffer (Promega) with water.

\section{Firefly luciferase assay buffer}

Tricine

$\left(\mathrm{MgCO}_{3}\right)_{4} * \mathrm{Mg}(\mathrm{OH})_{2} * 5 \mathrm{H}_{2} \mathrm{O}$

$\mathrm{MgSO}_{4}$

EDTA

DTT

Coenzym A

D-Luciferin

ATP

Keep in dark at $-20^{\circ} \mathrm{C}$.

Renilla luciferase assay buffer

$\mathrm{NaCl}$

$\mathrm{Na}_{2}$-EDTA

$\mathrm{KxPO}_{4}(\mathrm{pH} 5,1)$

Coelenterasin (dissolved in ethanol)
$20 \mathrm{mM}$

$1,07 \mathrm{mM}$

2,67 $\mathrm{mM}$

$0,1 \mathrm{mM}$

$33,3 \mathrm{mM}$

$270 \mu \mathrm{M}$

$470 \mu \mathrm{M}$

$530 \mu \mathrm{M}$

Keep coelenterasin stock at $-20^{\circ} \mathrm{C}$. To produce $\mathrm{KxPO}_{4}(\mathrm{pH}, 1)$ make $1 \mathrm{M} \mathrm{KH}_{2} \mathrm{PO}_{4}$ solution, then adjust $\mathrm{pH}$ level to 5,1 with $\mathrm{KOH}$.

After mixing the buffer components adjust $\mathrm{pH}$ to 5,0 ; keep in dark at $-20^{\circ} \mathrm{C}$.

\section{4\% paraformaldehyde solution}

Pre-warm $450 \mathrm{ml} \mathrm{H}_{2} \mathrm{O}$ to $65^{\circ} \mathrm{C}$, add paraformaldehyde $(20 \mathrm{~g})$, mix, add $\mathrm{NaOH}$ dropwise until solution becomes clear then adjust $\mathrm{pH}$ with $1 \mathrm{~N} \mathrm{HCl}$ to $\mathrm{pH} 7.4$, add $50 \mathrm{ml}$ 10x PBS, cool down to $4^{\circ} \mathrm{C}$, if necessary sterile filtrate $0.45 \mu \mathrm{m}$.

\section{Proteinase K buffer}

TrisCl $\mathrm{pH} 8$

EDTA pH 8

\section{0x SSC stock solution}

$\mathrm{NaCl}$

Na-Citrate $\left(\mathrm{Na}_{3} \mathrm{C}_{6} \mathrm{H}_{5} \mathrm{O}_{7} \times 2 \mathrm{H}_{2} \mathrm{O}\right)$

Adjust $\mathrm{pH}$ to 7,0 with $1 \mathrm{M}$ HCL.
$100 \mathrm{mM}$

$50 \mathrm{mM}$

$2,2 \mathrm{mM}$

$0,22 \mathrm{M}$

$1,43 \mathrm{mM}$ 


\section{Hybridization buffer (in situ hybridization)}

Formamide $50 \%$

Blocking agent (Boehringer)

$2 \%$

SSC

$5 \mathrm{x}$

SDS

$0,02 \%$

Sarcosyl

$0,1 \%$

Dissolve at RT for 30 minutes, then sterile filtrate. Store in aliquots at $-20^{\circ} \mathrm{C}$.

Maleic acid buffer (10x)

Maleic acid

$\mathrm{NaOH}$

$\mathrm{NaCl}$

Adjust $\mathrm{pH}$ to 7,5 ; sterile filtrate.

\section{Colour development buffer}

Tris $\mathrm{Cl} \mathrm{pH} 9,5$

$\mathrm{NaCl}$

$\mathrm{MgCl}_{2}$

$50 \mathrm{mM}$

Sterile filtrate.

\section{BCIP/X phosphate}

Stock solution $50 \mathrm{mg} / \mathrm{ml}$ in $100 \% \mathrm{DMF}$, store at $-20^{\circ} \mathrm{C}$.

NBT

Stock solution $75 \mathrm{mg} / \mathrm{ml}$ in $70 \% \mathrm{DMF}$, store at $-20^{\circ} \mathrm{C}$.

Working solution:

Shortly before use add $450 \mu \mathrm{l}$ of NBT stock solution and $350 \mu \mathrm{l}$ of BCIP stock solution to $100 \mathrm{ml}$ of colour development buffer. Keep in the dark.

TE (Tris-EDTA) buffer

Tris $\mathrm{Cl} \mathrm{pH} 7,5$

$10 \mathrm{mM}$

EDTA

$1 \mathrm{mM}$

TBS (tris-buffered saline) for immunocytochemistry

Tris, $\mathrm{pH} 7,5$

$25 \mathrm{mM}$

$\mathrm{NaCl}$

$136 \mathrm{mM}$

$\mathrm{KCl}$

$2.6 \mathrm{mM}$

Permeabilization buffer for immunocytochemistry

$0,1 \%$ Saponin in TBS

Blocking buffer for immunocytochemistry

$2 \%$ BSA, $0,1 \%$ porcine skin gelatine, $2 \%$ goat serum, $0,02 \%$ biotin, $0,1 \%$ saponin in TBS. 
Antibody dilution buffer for immunocytochemistry

$2 \%$ BSA, $0,1 \%$ porcine skin gelatine, $2 \%$ goat serum, $0,02 \%$ biotin, $0,02 \%$ saponin in TBS.

Citrate buffer for immunohistochemistry (antigen retrieval)

Solution A:

Citric acid $\mathrm{C}_{6} \mathrm{H}_{8} \mathrm{O}_{7} \quad 0,1 \mathrm{M}$

Solution B:

Sodium citrate $\mathrm{C}_{6} \mathrm{H}_{5} \mathrm{Na}_{3} \mathrm{O}_{7} \quad 0,1 \mathrm{M}$

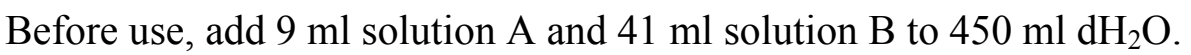

Tris buffer for immunohistochemistry (washing)

Tris $\mathrm{pH} 7,6 \quad 0,5 \mathrm{M}$

Working solution: add $9 \mathrm{~g} \mathrm{NaCl}$ to $100 \mathrm{ml}$ Tris buffer, add water to 11.

Tris buffer with milk: add 20 g non-fat dry milk powder to 11 working solution.

$\beta$-galactosidase staining buffer

$\mathrm{X}-\mathrm{Gal}(20 \mathrm{mg} / \mathrm{ml}$ in DMSO)

K-Ferricyanid (200 $\mathrm{mM}$ in PBS)

K-Ferrocyanid (200 $\mathrm{mM}$ in PBS)

$\mathrm{MgCl}_{2}(2 \mathrm{M})$

PBS

For whole mount embryos add:

$\mathrm{Na}$ Desoxycholate $(5 \%)$

NP40 (20\%)
$600 \mu 1$ (f.c. $1.2 \mathrm{mg} / \mathrm{ml}$ )

$250 \mu \mathrm{l}$ (f.c. $5 \mathrm{mM}$ )

$250 \mu \mathrm{l}$ (f.c. $5 \mathrm{mM}$ )

$10 \mu \mathrm{l}$ (f.c. $2 \mathrm{mM}$ )

To $10 \mathrm{ml}$

$20 \mu \mathrm{l}$ (f.c. $0,01 \%$ )

$10 \mu \mathrm{l}$ (f.c. $0,02 \%$ )

Thionin staining solution for cryosections

$\mathrm{NaAc}$, waterfree

Add water to

Thionin
$1,36 \mathrm{~g}$

$250 \mathrm{ml}$

$0,5 \mathrm{~g}$ per $100 \mathrm{ml}$ of solution, prepare fresh 


\section{METHODS}

\section{Molecular biology methods}

\subsection{Molecular cloning}

DNA digestion was performed with the help of Type II DNA endonucleases at $37^{\circ} \mathrm{C}$ in reaction volume of 20 to $100 \mu$ l. Choice of 10x reaction buffer was determined by the supplier's recommendations. DNA fragments were separated by electrophoresis in 1-2\% ethidium bromidecontaining agarose gel in 1x TAE buffer and visualized in the UV light. For using the fragments in further cloning, corresponding gel fragment were dissected by scalpel and DNA extraction was performed with the help of Gel extraction kit (Qiagen) according to manufacturer's protocol. In some cases, DNA inserts were phosphorylated before ligation (T4 polynucleotide kinase, 2 hours at $37^{\circ} \mathrm{C}$ followed by 10 minute heat-inactivation at $65^{\circ} \mathrm{C}$ ). Ligation of DNA fragments was performed with the help of T4 ligase (Promega) overnight at $+4^{\circ} \mathrm{C}$ or for 1 hour at RT. Generally, a vector to insert ratio of 1:4 was used. Ligation mix was transformed into bacteria using the Okayama transformation protocol for XL-1 blue strain or electroporation for DH5 $\alpha$ and DH10B strains.

\subsubsection{DNA transfer into bacteria}

\section{Okayama transformation}

Aliquots of transformation-competent bacteria were thawed on ice and transferred into clean vials containing 1,7 $\mu \mathrm{l} \beta$-mercaptoethanol per $100 \mu \mathrm{l}$ bacterial suspension. After 10 minute incubation the ligation mix was added to the vials, and bacteria were left on ice for additional 30 minutes, after which $30-40$ seconds heat shock was performed $\left(42^{\circ} \mathrm{C}\right)$. Bacteria were put on ice again for 2 minutes, then $800 \mu \mathrm{l}$ pre-warmed LB medium was added to each tube, and bacteria were allowed to recover for 45 minutes at $37^{\circ} \mathrm{C}$, moderate shaking, after which they were plated on relevant antibiotic-containing LB plates and incubated overnight at $37^{\circ} \mathrm{C}$.

\section{Electroporation of bacteria}

Aliquots of electrocompetent DH5 $\alpha$, DH10B or EL250/350 bacteria were thawed on ice and mixed with ligation mixes or plasmid DNA, after which the samples were transferred into $0,1 \mathrm{~cm}$ gap BioRad electroporation cuvettes and electroporated in BioRad "GenePulser" with the following parameters: voltage $1,8 \mathrm{kV}$, capacitance $25 \mu \mathrm{F}$, resistance $200 \Omega$. The cells were then resuspended in $800 \mu \mathrm{LB}$ medium, incubated for $30-45$ minutes at $37^{\circ} \mathrm{C}$ in an orbital shaker (EL250/350 were incubated for 90 minutes at $32^{\circ} \mathrm{C}$ ), then plated on relevant antibioticcontaining LB-plates and incubated overnight at $37^{\circ} \mathrm{C}$.

\section{Preparation of chemical competent cells (XL-1 blue)}

Bacteria were incubated in TB (terrific broth) medium at $18^{\circ} \mathrm{C}$ until the optical density of 0,6 was reached $(600 \mathrm{~nm})$ and then centrifuged 10 minutes at $3000 \mathrm{rpm}$ at $4^{\circ} \mathrm{C}$. After washing with TBjap, bacteria were again resuspended in TBjap, to which DMSO was added to the final concentration of $7 \%$. After waiting on ice for 10 minutes, bacterial suspension was aliquoted in eppendorf safe-lock tubes and snap-frozen in liquid nitrogen. 


\section{Preparation of electrocompetent bacteria}

Bacteria were allowed to grow in LB medium at $37^{\circ} \mathrm{C}$ until the optical density reached $0,5(600 \mathrm{~nm})$, after which they were centrifuged for 10 minutes at $3000 \mathrm{rpm}$. The supernatant was discarded, and the bacterial pellet was washed several times with ice-cold water. For the final three washes ice-cold water containing $10 \%$ glycerol was used. Bacteria were resuspended in $10 \%$ glycerol, aliquoted and snap-frozen in liquid nitrogen.

\subsubsection{Homologous recombination in bacteria}

In recent years recombination systems have been developed that utilize phage-derived factors enabling a site-specific recombination without the restriction-ligation-based methodology (Zhang et al., 1998; Lee et al., 2001). This method is also called "recombineering" and has been well described in literature (Zhang et al., 1998; Muyrers et al., 2000; Yu et al., 2000; Liu et al., 2003).

Efficient homologous recombination in $E$. coli is made possible by the use of $\lambda$-phage encoded Red genes. Copeland and colleagues (Lee et al., 2001) constructed several bacterial strains that carry a defective $\lambda$-prophage encoding the Red proteins exo, bet and gam, which have been placed under the transcriptional control of a temperature-sensitive $\lambda$-repressor. Exo encodes a 5'-3' exonuclease that acts on 5'ends of linear dsDNA fragments to produce 3' ssDNA overhangs; bet encodes a pairing protein that binds to 3' overhangs produced by exo and promotes their annealing to the complementary strands on the target DNA. The gam protein inhibits the RecBCD exonuclease activity of $E$. coli thereby protecting the linear dsDNA targeting DNA from degradation. By shifting the growth temperature from $32^{\circ} \mathrm{C}$ to $42^{\circ} \mathrm{C}$ for $10-$ 15 minutes the temperature-sensitive $\lambda$-repressor is no longer able to bind to the PL promoter allowing the transcription of exo, bet and gam at high levels. This system works very efficiently so that homology arms of 50-70 bp are already sufficient for homologous recombination.

The bacterial strains EL250 and EL350 also carry inducible cre and frt site-specific recombinases under the transcriptional control of the PвAD promoter, which allows their expression control by adding L-arabinose $(0.5 \% \mathrm{w} / \mathrm{v})$ to the LB medium. The recombinases were used to excise selectable markers, such as the zeocin resistance or kanamycin resistance, which were either flanked by loxP or FRT sites.

In this study $65 \mathrm{bp}$ homology arms were added to the targeting construct by PCR followed by sequence verification. Recombination was performed in the strain EL250. The bacteria were transduced with the BAC RP23-268011 (BAC PAC resources), containing NEX genomic region. From BAC-containing colonies electrocompetent cells were prepared, and subsequently electroporated with htTA cassette (kindly provided by R. Sprengel) bearing the homology arms, released from pGEMTeasy vector. Bacteria were grown at $42^{\circ} \mathrm{C}$ for 15 minutes to allow for homologous recombination and then plated on kanamycin agar plates to enable only the BAC-htTA cassette containing colonies' growth. After recombination was confirmed by PCR, the recombined colonies were expanded and grown in $0,1 \%$ arabinose containing medium, allowing for kanamycin-resistance cassette excision. BAC integrity was confirmed by various digests and consecutive pulse-field gel electrophoresis.

\subsection{Preparation and analysis of nucleic acids}

Small scale (mini-prep) and large scale (midi and maxi-prep) preparation of plasmid DNA was performed using the correspondent Qiagen kits. The principle of this DNA isolation is based on an alkaline lysis procedure (Birnboim and Doly, 1979) coupled to anion exchange resin purification under appropriate low-salt and $\mathrm{pH}$ conditions. Plasmid DNA was eluted in distilled water. DNA preparation for further primary culture transfection was performed with the Qiagen Endo-free Maxi Kit. Bacterial endotoxins, also known as lipopolysaccharides or LPS, strongly 
influence transfection of DNA into primary cells. The Qiagen Endo-free DNA preparation procedure couples plasmid DNA isolation with endotoxin removal by incubation with endotoxinremoval buffer that prevents LPS molecules from binding to the QIAGEN tips resin.

\subsubsection{BAC DNA preparation}

\section{Small scale preparation of bacterial artificial chromosomal DNA, "BAC minis"}

For the purification of small amounts of BAC DNA for BAC fingerprinting a modified alkaline lysis procedure was used (Birnboim and Doly, 1979). In brief, $4 \mathrm{ml}$ of LB medium with the appropriate antibiotic were inoculated from a single BAC clone or glycerol stock and grown overnight at $37^{\circ} \mathrm{C}$ or $32^{\circ} \mathrm{C}$ (for bacterial strains EL250, EL350). $2 \times 1,5 \mathrm{ml}$ of overnight culture were then spun down into the same tube, the supernatant was discarded and the pellet was resuspended in $400 \mu 1$ of buffer P1 (Qiagen). The cells were lysed by adding $400 \mu 1$ of buffer P2 (Qiagen) and proteins and bacterial chromosomal DNA were precipitated by adding $400 \mu 1$ of chilled buffer P3 (Qiagen). The cellular debris was removed by centrifugation and the supernatant was transferred into a new tube. BAC DNA was then precipitated by adding $450 \mu 1$ ice-cold isopropanol followed by an incubation at $-20^{\circ} \mathrm{C}$ for 1 hour. The BAC DNA was pelleted by centrifugation $(13.000 \mathrm{~g}, 10$ minutes) in a table-top centrifuge. The supernatant was discarded and the DNA was washed once with $1 \mathrm{ml}$ of $80 \%$ ethanol, air dried and re-suspended in $40 \mu \mathrm{l}$ of BAC-TE.

\section{Large-scale BAC DNA preparation (BAC maxi)}

Larger amounts of BAC DNA were obtained by an alkaline lysis procedure followed by anion-exchange resin chromatography (NucleoBond kit, Clontech). $250 \mathrm{ml}$ of selective LB medium were inoculated with 2,5 ml of an BAC overnight culture and grown for 18 hours at the appropriate temperature. Cells were spun down and either frozen away or directly processed. Cells were re-suspended in $12 \mathrm{ml}$ of chilled buffer S1, lysed by adding $12 \mathrm{ml}$ of buffer S2. Due to the large amount of cells, the lysis time was extended to 5 minutes. $12 \mathrm{ml}$ of chilled buffer S3 were added and the lysate was cleared by filtration through a pre-wetted filter. The lysate was then passed onto a AX-500 tip, which was pre-equilibrated with $5 \mathrm{ml}$ of buffer $\mathrm{N} 2$ followed by two $12 \mathrm{ml}$ washes with buffer N3. The BAC DNA was eluted from the column by two $3 \mathrm{ml}$ elution steps with buffer $\mathrm{N} 5$ heated to $55^{\circ} \mathrm{C}$. The DNA was precipitated by adding $8,4 \mathrm{ml}$ of isopropanol and pelleted by centrifugation at $12.000 \mathrm{~g}$ for 25 minutes. The BAC DNA pellet was then washed twice with $4 \mathrm{ml}$ of $80 \%$ ethanol, air dried and re-suspended in 200-300 $\mu$ l of BACTE.

\subsubsection{DNA purification for injection}

Transgene vectors were digested to remove the vector backbone, and the transgene fragments were extracted from agarose gel (no ethidium bromide was added to the gel or electrophoresis buffer). After ethanol precipitation the DNA pellet was resuspended in the TrisEDTA injection buffer to the final concentration of $200 \mathrm{ng} / \mu \mathrm{l}$.

NEX-htTA BAC was isolated from bacteria using the BAC maxi protocol and resuspended in the Tris-EDTA injection buffer.

\subsubsection{Genomic DNA extraction from mouse tissues}

Genomic DNA preparation was done with the help of Invitek Mini Tissue Kit, combining cell lysis with column purification. Briefly, mouse tail or brain tissue was digested with proteinase $\mathrm{K}$ in lysis buffer $\mathrm{G}$ overnight at $52^{\circ} \mathrm{C}$. After mixing with binding buffer $\mathrm{T}$ the lysate 
was transferred to the DNA-purification column and centrifuged. The column was washed twice and genomic DNA was eluted in pre-warmed elution buffer.

\subsubsection{Polymerase chain reaction (PCR)}

The polymerase chain reaction allows the in vitro amplification of a specific DNA sequence (Mullis et al., 1986). The DNA synthesis is started at two primers that are flanking the sequence to be amplified. One of the primers anneals to the sense and the other one to the antisense strand of the amplicon. The synthesis reaction is catalyzed by the DNA polymerase of the thermophilic bacterium Thermus aquaticus (taq polymerase). The enzyme is stable at $95^{\circ} \mathrm{C}$ and catalyzes the synthesis of DNA at a temperature of $72^{\circ} \mathrm{C}$. DNA synthesis therefore can be achieved in a thermocycler shifting temperatures between $95^{\circ} \mathrm{C}$, the specific annealing temperature of the primers and $72^{\circ} \mathrm{C}$ for DNA synthesis in the presence of a DNA template, primers, dNTPs and corresponding salt and $\mathrm{pH}$ conditions for taq polymerase.

Usually, 24 - 40 cycles are necessary to obtain sufficient quantities of the PCR product for subsequent steps. For this thesis Qiagen's @HotStar taq polymerase"was used, which is inactive at ambient temperatures to avoid misprimed PCR products. The "Master Mix" also contains dNTPs and a proprietary buffer. The enzyme can be activated by an initial denaturing step at $95^{\circ} \mathrm{C}$ for 15 minutes.

Standard PCR reactions were setup as follows:

$2 \mu 1$ 10x Buffer

$2 \mu \mathrm{dNTPs}(2 \mathrm{mM})$

$0,5 \mu$ primer $1(10 \mathrm{pM})$

$0,5 \mu 1$ primer2 $(10 \mathrm{pM})$

$1 \mu$ l DNA template $(100 \mathrm{pg}-25 \mathrm{ng})$

$14 \mu 1$ PCR-grade $\mathrm{dH}_{2} \mathrm{O}$

0,3-0,5 $\mu 1$ Taq polymerase

$20 \mu 1$

PCR was also used to introduce new restriction sites into the amplified product. The new restriction sites to be added were included in the sequence of PCR primers used. Taq polymerase does not possess a 3'-5' exonuclease activity ("proofreading"), which on average leads to the introduction of a mutation every $1.3 \times 10^{5}$ nucleotides. For high-fidelity amplifications, the DNA polymerase of Pyrococcus furiosus (pfu polymerase) was used, which has a proofreading activity that results in a $>10$-fold reduced mutation rate.

\subsubsection{In vitro transcription}

In vitro transcription was used for synthesis of DIG-labelled cRNA probes for in situ hybridization. The following reaction setup was used:

$4 \mu \mathrm{l}$ linearized DNA template $(250 \mathrm{ng} / \mu \mathrm{l})$

$6,5 \mu 1 \mathrm{H}_{2} \mathrm{O}$

$4 \mu 15 x$ transcription buffer (Promega)

$2 \mu 1100 \mathrm{mM}$ DTT (f.c. $10 \mathrm{mM}$ )

$2 \mu 1$ 10x DIG-RNA mix (Boehringer)

$0,5 \mu 1$ RNAsin $40 \mathrm{U} / \mu 1$

$1 \mu 1 \mathrm{~T} 3 / \mathrm{T} 7 / \mathrm{Sp} 6$ RNA-polymerase

$20 \mu 1$ 
The mix was incubated for $2-6$ hours at $37^{\circ} \mathrm{C}$. To remove the template DNA and to stop the reaction $2 \mu \mathrm{RQ} 1-\mathrm{DNase}$ was added. After 15 minutes incubation at $37^{\circ} \mathrm{C} 1 \mu 10,5 \mathrm{M}$ EDTA $(\mathrm{pH} 8,0)$ was added and the reaction was put on ice. Labelled RNA was then precipitated by ethanol and resuspended in RNase-free water. After determining the final RNA concentration at the spectrophotometer and RNA quality check on agarose gel the probes were stored at $-20^{\circ} \mathrm{C}$ either in water or in hybridization buffer.

\subsubsection{Total RNA extraction from mouse tissues}

Mouse brain, brain parts or embryos were put into pre-cooled vials after dissection and homogenized for 15 - 30 seconds in $2 \mathrm{ml}$ eppendorf tubes in RLT buffer (Qiagen) with the help of Polytron rotor-stator tissue homogenizer. RNA isolation followed Qiagen RNeasy Mini Kit protocol. Briefly, lysate was centrifuged, and the supernatant was mixed 1:1 with $70 \%$ ethanol and applied to the RNA isolation column. After centrifugation the column was washed twice, and RNA was eluted in 30-100 $\mu$ l of RNase-free water.

The laser-captured CA1 and CA3 regions of hippocampus were stored in RNAlater buffer at $-80^{\circ} \mathrm{C}$ until RNA isolation. The samples were thawed and vortexed, and the RNA isolation was performed using Qiagen RNeasy Micro Kit. The eluted in $100 \mu 1$ water RNA samples were precipitated in ethanol at $-20^{\circ} \mathrm{C}$, resuspended in $2 \mu \mathrm{l}$ RNase-free water and immediately used for cDNA synthesis.

\subsubsection{Reverse transcription and quantitative real-time PCR}

Reverse transcription (RNA-dependent DNA synthesis) was performed on mouse brain total RNA, mouse hippocampal slices total RNA and on the RNA isolated from laser-captured CA1 and CA3 areas. In all applications generation of the total cDNA pool was required, therefore random nonamer primer mix was used for amplification.

Reverse transcription reaction was performed with the help of SuperScript ${ }^{\mathrm{TM}}$ III RNaseHKit (Invitrogen) according to the manufacturer's protocol. Briefly, $2 \mu$ l of template RNA was mixed with $3 \mu \mathrm{l}$ of random nonamer primer mix $(25 \mathrm{pm} / \mu \mathrm{l})$ and $11 \mu \mathrm{l}$ water and heated at $70^{\circ} \mathrm{C}$ for 10 minutes. Then the reaction mix was put on ice and $4 \mu 1$ of $5 \mathrm{x}$ reaction buffer, $1 \mu 1$ of $10 \mathrm{mM}$ dNTPs and $1 \mu \mathrm{l}$ of $100 \mathrm{mM}$ DTT were added to the reaction tube. Following 10 minutes incubation at $42^{\circ} \mathrm{C}, 1 \mu \mathrm{l}$ of SuperScript reverse transcriptase was added and the final reaction mix was incubated for 10 minutes at $25^{\circ} \mathrm{C}$, then 55 minutes at $42^{\circ} \mathrm{C}$ and 15 minutes at $70^{\circ} \mathrm{C}$, after which the reaction tube was put on ice.

cDNA was then further used in a quantitative real-time PCR for determining the relative expression profiles. Quantification of the PCR products was performed with the help of the Fast Real-Time PCR System (Applied Biosystems), which measures the level of intercalation of SYBR-Green dye into the reaction product. The values of the genes of interest were normalized to $\beta$-actin and 18sRNA levels. Specific qPCR primers were designed with the help of Roche Universal Probe Library software (www.roche-applied-science.com).

Each real-time PCR reaction contained:

$5 \mu 1$ SYBR-Green Master Mix

$0,1 \mu 1$ forward primer

$0,1 \mu 1$ reverse primer

$0,8 \mu 1 \mathrm{H}_{2} \mathrm{O}$

$4 \mu 1 \mathrm{cDNA}$ (various dilutions)

$10 \mu 1$ 


\subsubsection{Photometric determination of nucleic acid concentration}

According to the Lambert-Beer law the absorption of an aqueous solution of a substance is directly proportional to its concentration, $A=\varepsilon \mathrm{cl}$, where $\varepsilon$ is the molar extinction coefficient (unit: M-1cm-1), c the concentration (unit: M) and 1 is the path length of the light through the sample (unit: $\mathrm{cm}$ ). The extinction coefficients for nucleic acids are (at $\lambda=260 \mathrm{~nm}$ ):

guanine : $\varepsilon=12010 \mathrm{M}-1 \mathrm{~cm}-1$

cytosine : $\varepsilon=7050 \mathrm{M}-1 \mathrm{~cm}-1$

adenine : $\varepsilon=15200 \mathrm{M}-1 \mathrm{~cm}-1$

thymine/uracil : $\varepsilon=8400 \mathrm{M}-1 \mathrm{~cm}-1$ ( $8111 \mathrm{M}-1 \mathrm{~cm}-1$ for uracil )

The absorption was measured at $260 \mathrm{~nm}$ and $280 \mathrm{~nm}$, which represent the absorption maxima for nucleic acids and proteins, respectively. The measured absorption is the sum of the absorptions of all the bases in the solution. For working purposes the following OD260 concentration relations were used:

1 OD260 for dsDNA : $50 \mathrm{mg} / \mathrm{ml}$

1 OD260 for RNA : $40 \mathrm{mg} / \mathrm{ml}$

1 OD260 for ss-oligonucleotides : $33 \mathrm{mg} / \mathrm{ml}$

The purity of a given sample was assessed by the ratio OD260:OD280. For clean preparations of nucleic acids, the ratio should be between 1,7 and 2,0; lower ratios usually indicate a protein contamination. Depending on the expected concentration of a sample it was diluted 1:50 to $1: 1000$ prior to measuring.

\subsection{Preparation and analysis of proteins}

\subsubsection{Protein extraction from cultured cells}

For total cytoplasmic and nuclear protein pools isolation cells were washed with PBS and lysed in the LyB for 5 minutes on ice. With the help of cell scraper, the lysate, containing cytoplasm, membrane pieces and intact nuclei, was collected into an eppendorf tube and centrifuged at $2000 \mathrm{rpm}$ for 10 minutes at $4^{\circ} \mathrm{C}$. The supernatant (cytoplasmic proteins) was collected into a separate cryovial and snap-frozen in liquid nitrogen. The pellet was resuspended in NLysB and incubated for 1 hour at $4^{\circ} \mathrm{C}$ and subsequently centrifuged at $16000 \mathrm{rpm}$ for 15 minutes at $4^{\circ} \mathrm{C}$. The supernatant (containing nuclear proteins) was transferred into a separate cryovial and snap-frozen in liquid nitrogen.

\subsubsection{Protein extraction from mouse tissues}

Mouse brain, brain parts or embryos were put on ice after dissection and homogenized for 60 seconds in $2 \mathrm{ml}$ eppendorf tubes in TBS buffer using a Polytron rotor-stator tissue homogenizer and centrifuged for 5 minutes at $4^{\circ} \mathrm{C}$. Pellet was resuspended in LysB and left on ice for 15 minutes, after which $50 \mu$ l of TritonX100 were added to the lysate followed by brief vortexing and centrifugation at $2000 \mathrm{rpm}$ for 10 minutes at $4^{\circ} \mathrm{C}$. The pelleted nuclei were resuspended in NLysB and incubated at $4^{\circ} \mathrm{C}$ for 1 hour and then centrifuged at $13000 \mathrm{rpm}$ for 20 minutes at $4^{\circ} \mathrm{C}$. The supernatant was transferred into a fresh tube and snap-frozen in liquid nitrogen.

\subsubsection{Protein concentration measurement (Bradford assay)}

This assay was first introduced by Bradford and is a quick and reliable means of determining the protein concentration of a given solution (Bradford, 1976). Basic amino acid side chains of proteins form complexes with Coomassie Brilliant Blue G-250 in solution that appear blue with an absorption maximum at $\lambda=595 \mathrm{~nm}$. The absorption is proportional to the 
amount of protein in solution. Through comparison with a calibration curve the protein concentration in a sample can be determined photometrically by measuring its OD595. Calibration curves have to be generated for each assay with defined protein concentrations. The interactions of Coomassie Brilliant Blue G-250 and the amino acid side chains are detergent sensitive; thus detergent concentrations of $>1 \%$ in the assay should be avoided.

Usually, a Bradford assay was setup as follows:

1-10 $\mu$ l protein lysate

$\mathrm{dH}_{2} \mathrm{O}$ to $40 \mu \mathrm{l}$

$+160 \mu 1$ Bradford reagent

The mix was incubated for 20 minutes at RT prior to measuring OD595. If the OD595 was $>1$, the sample was diluted further and the assay was repeated.

\subsubsection{Polyacrylamide gel electrophoresis (SDS-PAGE)}

Unlike DNA, proteins do not have a constant mass-charge ratio due to their differing amino acid sequence. Through heat-denaturing of the protein in the presence of the detergent sodium dodecylsulfate (SDS), the detergent is able to form a 'micelle' with the unfolded protein as its core. The length of this structure is proportional to the polypeptide chain length and therefore to its molecular weight. In addition to SDS, a reducing agent like $\beta$-mercaptoethanol or DTT is added to reduce any disulfide bonds.

The Invitrogen NuPAGE $\AA$ Bis-Tris system, used in this study, is based on a Bis-Tris$\mathrm{HCl}$ buffered polyacrylamide gel $(\mathrm{pH} 6,4)$. The Bis-Tris gels do not contain SDS, so they were used in the denaturing conditions. Protein samples were denatured in the NuPAGE® LDS loading buffer $\left(75^{\circ} \mathrm{C}, 10\right.$ minutes), loaded onto the $4 \%-12 \%$ gradient gel, and electrophoresis was performed at $100 \mathrm{~V}$ until the desired degree of protein separation was achieved.

\subsubsection{Western blotting}

The protein transfer from polyacrilamide gel onto PVDF membrane was carried out in the Invitrogen "XCell II" wet blotting chamber. The blot was set up as follows:

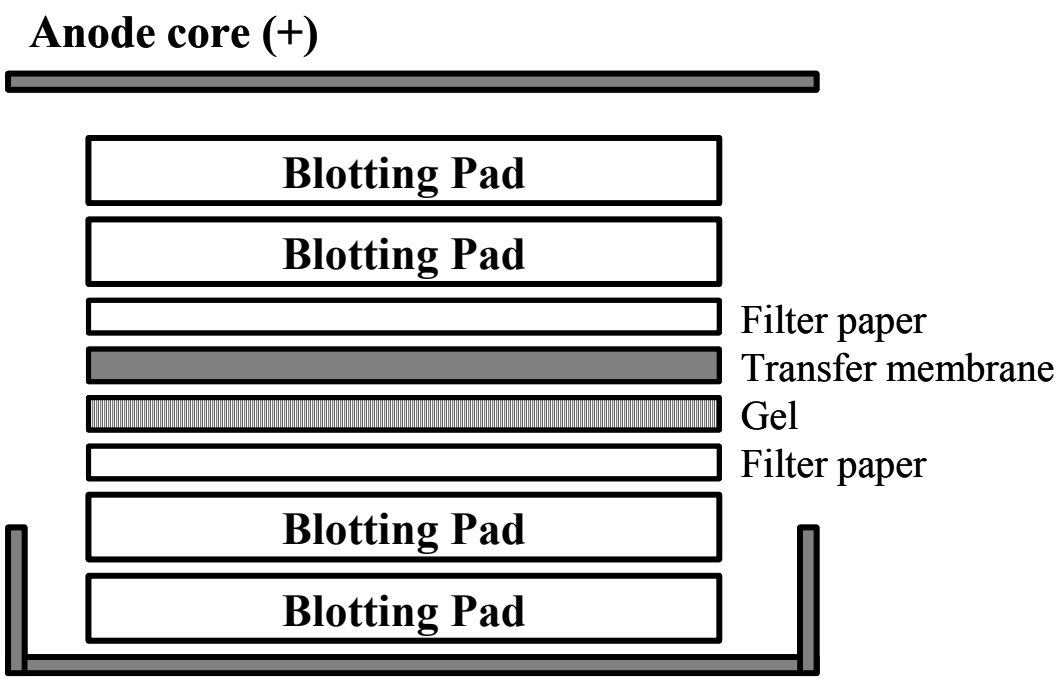

\section{Cathode core (-)}

Before blotting the PVDF membrane was soaked in methanol for 5 minutes and washed in transfer buffer. The sponges and filter paper were pre-wetted in transfer buffer. When an 
electric current is applied to the XCell blotting module, the negatively charged proteins migrate from the cathode to the anode and are retained on the PVDF membrane by electrostatic and hydrophobic interactions. For the transfer the blotting chamber was placed in a bucket with ice to avoid heating of the setup. Transfer was performed at $150 \mathrm{~mA}$ and a maximum of $35 \mathrm{~V}$ for approx. 4 hours. The blot was then disassembled, the membrane was soaked briefly in methanol, washed twice in TBST and placed in blocking buffer for 1 hour at RT or overnight at $4^{\circ} \mathrm{C}$. The primary antibody was diluted in antibody dilution buffer (blocking buffer without Tween20) and incubated with the membrane for 1-2 hours at RT followed by four 15 minute washes in TBST. The secondary HRP-coupled antibody, diluted in antibody dilution buffer, was then applied to the membrane and incubated for 45 minutes at RT followed by four 15 minute washes in TBST.

ECL solution (PerBio) was pre-warmed to RT and applied onto the membrane for 1 minute according to the manufacturer's recommendations. The membrane was then covered with Saran wrap and exposed to an ECL hyperfilm (Amersham). The exposure times varied depending on the signal intensity. The films were then developed in a KODAK X-OMAT developer. For re-probing of the membrane with another antibody, the membrane was placed into Western Blot stripping buffer at $60^{\circ} \mathrm{C}$ for $1-2$ hours in a water bath followed by three 15 minute washes with TBST.

\section{Tissue culture}

\subsection{Culture and transfection of mammalian cell lines}

Frozen aliquots of mammalian cell lines were transferred from the liquid nitrogen tank onto ice and allowed to thaw for 20-30 minutes, after which the cell suspension was diluted with access amount of serum-containing medium, washed and plated.

Cells were allowed to grow on $15 \mathrm{~cm}$ tissue culture dishes (Falcon) in $\mathrm{CO}_{2}$ tissue culture incubators at $37^{\circ} \mathrm{C}$ with $5 \% \mathrm{CO}_{2}$. When $70-90 \%$ confluency was reached, the cell layer was washed with PBS and incubated with Trypsin-EDTA for $1-5$ minutes at $37^{\circ} \mathrm{C}$, after which serum-containing medium was added, and the cell suspension was collected in a $15 \mathrm{ml}$ Falcon tube, centrifuged, resuspended in medium and transferred to fresh plates. No additional coating was used for COS1 cells, whereas for PC12 cells culture dishes, well plates and coverslips were coated with poly-L-lysine.

For transfection PC12 cells were plated onto coverslips in $24-w e l l$ plates $\left(10^{6}\right.$ cells/well) or onto 96-well plates $\left(5 \times 10^{4}\right.$ cells/well) and allowed to grow for 18 -24 hours, after which medium was changed to serum-free and DNA constructs were introduced by means of lipofection. For 24-well plates $0,5-1 \mu \mathrm{g}$ of DNA and $1 \mu \mathrm{l}$ Lipofectamine2000, and for 96-well plates 200-400 ng of DNA and 0,2 $\mu$ l Lipofectamine2000 was used. Cells were fixed or lysed 1824 hours after transfection.

COS 1 cells were electroporated in 0,4 cm BioRad cuvettes in BioRad "GenePulser" with the following parameters: voltage $1,7 \mathrm{kV}$, capacitance $25 \mu \mathrm{F}$, resistance $200 \Omega$. $2-3 \times 10^{6}$ cells were resuspended in $300 \mu \mathrm{l}$ electroporation buffer, containing 6-10 $\mu \mathrm{g}$ of DNA, and after electroporation plated onto $6 \mathrm{~cm}$ tissue culture dishes. Cells were lysed 24-48 hours later.

\subsection{Culture of mouse primary neurons}

Mouse E16 or E18 embryos were dissected and put into ice-cold HBSS+. After isolation of cortices and hippocampi, meninges were removed under binocular and neuronal tissues were transferred into $15 \mathrm{ml}$ Falcon tube. HBSS + was replaced with Trypsin-EDTA solution, and after resuspension of tissue DNaseI solution $(0,05 \%$ in $\mathrm{HBSS}+)$ was added. After 10 minutes incubation at RT neurons were again resuspended, washed with HBSS + and triturated again in DNaseI solution. After the final wash, neurons were resuspended in Neurobasal medium and 
plated onto poly-D-lysine coated coverslips in 24-well plates. Neuronal culture was incubated in $\mathrm{CO}_{2}$ tissue culture incubators at $37^{\circ} \mathrm{C}$ with $5 \%$ atmospheric $\mathrm{CO}_{2}$. Neurons were transfected with Lipofectamine2000 following the protocol used for PC12 cells and fixed 24 hours after transfection with 4\% PFA in PBS.

\subsection{Immunocytochemistry}

Cells were washed with PBS, fixed with $4 \%$ PFA for 10 minutes, washed 3 times with PBS and permeabilized with $0,1 \%$ saponin for 30 minutes at RT. Blocking was carried out for 30 minutes, after which primary antibody was applied for $1-2$ hours at RT or overnight at $4{ }^{\circ} \mathrm{C}$ in a humidified chamber. After three PBS washes secondary antibody-DAPI mix was applied for 45 minutes at RT. Cells were washed again in PBS and rinsed in distilled water, and coverslips were mounted onto glass slides with AquaPolyMount (Polysciences Inc).

\subsection{Luciferase reporter gene assay}

Luciferase reporter assay is based on the ability of luciferase protein to catalyze its substrate into a luminescent product in the presence of ATP. In this study the assay was performed according to the standard laboratory protocol (Wehr et al., 2006) on the MicrolumatPlus 96V luminometer (Berthold Technologies). Briefly, medium was removed from the transfected cells 24 hours after transfections and the cells were lysed in Passive Lysis Buffer (Promega) for 20 minutes at slow shaking. The lysates were then transferred into a fresh white 96-well plate and the luciferase activity in each well was measured in the luminometer. The following parameters were used:

- $75 \mu$ Firefly luciferase substrate, reaction time 1,6 seconds, luminescence intensity integration time 6 seconds, break 6 seconds;

- $75 \mu$ Renilla luciferase substrate, reaction time 2 seconds, luminescence intensity integration time 6 seconds.

Winglow software was used for further data analysis.

\section{Production and maintenance of transgenic mice}

All animal experiments used in this study were performed in compliance with animal policies of the Max-Planck-Institute of Experimental Medicine. DNA injection into pronuclei and embryo transfer was performed in the transgenic facility of the MPI of Experimental Medicine by Simone Schmidt and Monika Schindler. Potential founders were ear-marked and tail biopsy was taken at 4-6 weeks of age. 


\subsection{Enriched environment housing}

Typically, mice were housed in $19 \mathrm{~cm}$ wide, $34 \mathrm{~cm}$ long and $20 \mathrm{~cm}$ high standard mouse cages with straw, Kimberley-Clark wipes as nesting material and food and water ad libitum. The enriched environment cages were $36 \mathrm{~cm}$ wide, $50 \mathrm{~cm}$ long and $20 \mathrm{~cm}$ high, i.e. approx. 3 times larger than conventional cages. In addition, each enriched environment cage was equipped with a running wheel, a 2-floor house, a crunchy tunnel and a wall separating "living room" from "dining room" as shown below:

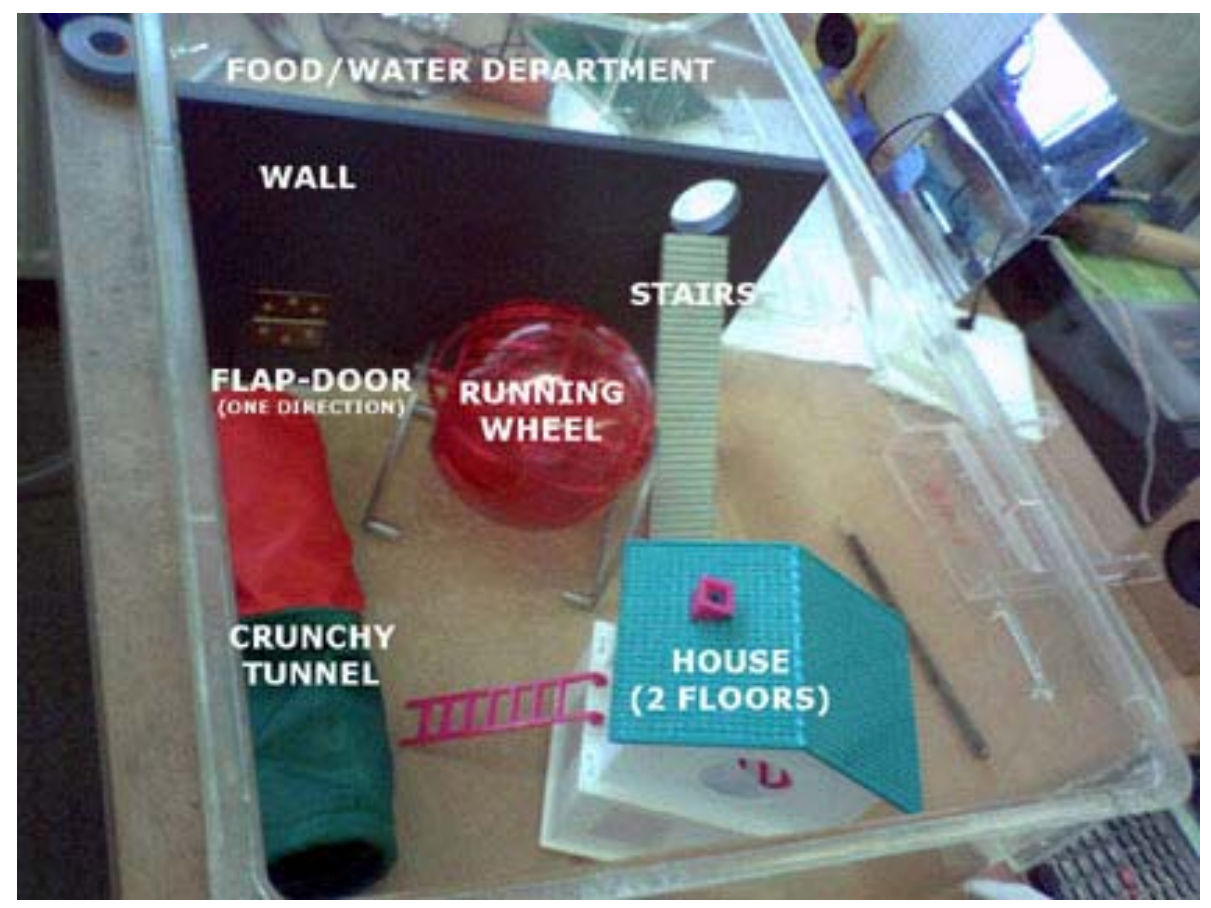

The mice had access to food and water compartment ("dining room") through the stairs, and could go back to the "living room" through a one direction flap-door.

Mice were housed in the enriched environment cages for 2 or 7 days. Every day a toilet paper roll carton and a novel object of yellow colour ("Easter chicken" or "Duck") were placed into the cage. Additional enrichment was provided by "handling" the mice: every day each mouse was taken into hands and allowed for a minute to explore the hands, watch and lab-coat sleeves of the experimentator. Thus all criteria of the environmental enrichment were met: novelty, physical exercise, cognitive, visual and somatosensory stimulation (Nithianantharajah and Hannan, 2006).

\subsection{Behavioural cohort breeding and maintenance}

Behavioural cohorts were comprised of wildtype and NEX null mutant littermates, all mice within one cohort born within a week. The first cohort contained exclusively male mice (14 null mutants and 10 wildtype controls) that were tested at the age of 5,5 to 7 months. The second cohort contained male and female mice of mixed genotypes ( 9 null mutants and 10 wildtype controls), tested at the age of 6 to 9 months. Mice were housed in regular cages in numbers of 3 to 6 , gender-separated, with individuals of both genotypes present in each cage. 


\subsection{Behavioural assays}

\subsubsection{Open field}

Spontaneous activity in the open field is the most general measure of mouse motor function. The automated open field boxes can be square or round shape and are supplied with photocell beams that detect movement. Typically the automated open field is a square box $40 \times 40 \mathrm{~cm}$ in size, where 8 photocell emitters along one side of the floor send out beams that are detected by the receptors on the opposite wall (x axis). Similarly, another set of emitters and receptors is located on the adjacent walls, thus forming the y axis. One more set of photocells is located at $4 \mathrm{~cm}$ level above the floor and constitutes the $\mathrm{z}$ axis. When the animal moves in the box the beam path is broken and it is detected by the receptor and recorded by the photocell analyzer software. Beam breaks of the lower photocell sets (x-y) are recorded as horizontal activity, whereas the $\mathrm{z}$ axis recordings reflect the animal standing upright in a vertical position (usually when exploring the walls), the so-called rearings. The computer calculates a large set of variables, such as horizontal activity, vertical activity, total distance travelled, total number of movements, resting and moving time, total number of rearings, rearings in each corner and time spent in the centre of the field versus time spent in the periphery.

In this study an automated $45 \times 45 \mathrm{~cm}$ open field box (TSE $\mathrm{GmbH}$ ) was used; each mouse was allowed to explore the box for 10 minutes, and scores of total distance travelled, time spent at rest, time spent moving, time spent in the periphery or in the centre of the field and the average velocity were taken for statistical analysis.

\subsubsection{Rotarod}

Ability to stay on a rotating cylinder, called rotarod, serves as a test for general motor coordination and balance. A typical mouse rotarod is $3-4 \mathrm{~cm}$ in diameter and rotates at a constant speed of 3-5 rpm. A mouse placed onto it has to walk forward in order to stay on the rotating cylinder; the latency to fall off is measured as the dependent variable (usually a cut-off time of 12 minutes is used). In many automated systems a timer is linked to the floor panel under the cylinder, onto which the mouse falls. Usually the mouse is given one or two practice trials, after which it is again placed onto the rod and the latency to fall is recorded. Modern automated rotarod systems allow simultaneous scoring of several animals.

The accelerating rotarod is used to assess motor learning in rodents. The mouse is placed onto the cylinder at 3-4 rpm, but then the rotation speed gradually rises to 30-40 rpm over a period of 3-5 minutes. Again the latency to fall off is recorded, and most mice fall off the rod well before the cut-off time. Since the accelerating rod is a challenging motor learning task, it is usually repeated on 3-5 consecutive days. Performance over days of repeated training is quantified as a measure of motor learning.

Accelerating rotarod (TSE GmbH), $3 \mathrm{~cm}$ in diameter, with rotation speed rising from 3 to $30 \mathrm{rpm}$ over a period of 5 minutes was used in this study. Mice were given several practice trials on the first day, after which each mouse had two recorded trials on four consecutive days.

\subsubsection{Light-dark preference box}

Light-dark preference test is used for measuring the general anxiety and exploratory activity of rodents. It usually consists of a box with a light (transparent ceiling and walls) and a dark compartment, separated by a door. The mouse is placed into the light compartment, and the time count starts after it crosses the wall and enters the dark compartment. Then the time spent in the dark box, the time spent in the light box and the number of crossings between the compartments is measured. 
In this study a $37 \times 24 \times 20 \mathrm{~cm}$ plexiglas box with $3 \times 5 \mathrm{~cm}$ door between compartments was used, and the mouse movements were scored manually for 5 minutes, starting from the first entrance into the dark compartment.

\subsubsection{Elevated plus maze}

Plus maze consists of two open arms and two closed arms, connected in a cross-shape with a small open square in the middle, and is used for measuring general anxiety and fear in mice. The elevated plus maze is elevated $20-30 \mathrm{~cm}$ above the floor; the mouse is placed onto the platform in the middle and allowed to move freely in the arms of the maze for 5-10 minutes scoring time. Time spent in the closed arms, time spent in the open arms and the number of crossings between closed and open arms is scored.

The elevated plus maze used in this study had $5 \mathrm{~cm}$ wide and $30 \mathrm{~cm}$ long arms, the closed arms having $15 \mathrm{~cm}$ high walls, and was elevated $25 \mathrm{~cm}$ above the floor. Each mouse was put into the middle and behaviour was scored manually for 5 minutes.

\subsubsection{Hot plate}

Hot plate test is a simple test to measure pain sensitivity. The mouse is put onto a platform heated to $52-56^{\circ} \mathrm{C}$ (depending on age of the mouse) and the latency to lick the hind paw is recorded. In this study platform heated to $54^{\circ} \mathrm{C}$ was used, and the mouse was taken away from the platform immediately after it licked one of its hind paws or jumped out. The mice that failed to do so were taken off the hot plate after a cut-off time of 60 seconds and excluded from the statistical analysis.

\subsubsection{Visual cliff test}

Vision acuteness was measured in mutant and wildtype mice with the help of visual cliff box (Crawley, 2000). The principle of vision assessment in this test lies in the optical illusion. The box contains a cliff of approximately $50 \mathrm{~cm}$; both upper and lower surfaces of which are covered with black and white chequered paper. Transparent plexiglas cover is then placed onto the upper surface, and the "virtually safe" and "virtually unsafe" parts are separated by a $0,5-1 \mathrm{~cm}$ high bar. The mouse is placed on the bar and allowed to step down. If the decision is based on visual stimuli, the mouse will typically step down to the "virtually safe" area; however, if the mouse is blind, it will rely on the somatosensory information from the whiskers and will step down to both sides of the cliff with equal probability.

In this study, each animal was given 10 step-down trials on the same day, and the percent share of "virtually safe" decisions was taken for the statistical analysis.

\subsubsection{Morris water maze}

The Morris water maze task is a widely used means to assess spatial navigation and spatial learning in rodents. The animal learns to find an escape platform, hidden under water surface, by orienting itself on the spatial cues present in the room. The paradigm was developed more then twenty years ago by Professor Morris (Morris, 1981; Morris, 1984) and is based on the assumption that rodents are highly motivated to escape from water by the quickest and most direct route.

The hidden platform finding task is often preceded by the visible platform finding task, in which mice are trained to swim to the platform, which is covered by water, but its location is marked with a flag. The visible platform training demonstrates the means of escape (i.e. the platform) to the animals and helps the mouse overcome the original stress it experiences by being placed into water. 
In the course of hidden platform training mice are placed in a round water tank filled with opaque water and are allowed to swim for 90 seconds, after which they are guided to the hidden platform, assisted in climbing it and left on the platform for 30 seconds. If the mouse finds the platform itself, the trial time stops, and the mouse is allowed to sit on the platform for 30 seconds before return to home cage. The animals use the time they spend on the platform to look around and memorize the environmental cues (usually 1-5 high-contrast objects are placed on the walls to assist spatial orientation), which allows them to find the platform faster in the following trials. Each mouse performs several trials per day over a period of 1-3 weeks, and the swimming trajectory, distance travelled and latency to find the platform are recorded.

The hidden platform training is often followed by reversal training, where the cues on the walls and the platform location are changed, and the mouse has to master the new task. An important part of the training is the so-called probe trial, which usually takes place the day after the training is completed; this procedure has a key role in evaluating whether the mouse has mastered the task. The platform is removed from the water tank, and the mouse is allowed to swim for 90 seconds. If the mouse has memorized the platform location, the time it spends in the target quadrant in the probe trial should be significantly higher than the time it spends in each of other three quadrants.

In this study a $120 \mathrm{~cm}$ in diameter water tank was used, white wall paint was mixed to the water to make the platform invisible, and the mouse trajectory was recorded by TSE VideoMot2 software (TSE GmbH), which received input from the camera hanging above the tank. Each mouse performed 4 (in some cases 8 ) trials per day and was put onto $37^{\circ} \mathrm{C}$-heated platform between trials to allow it to dry. Distance travelled and time spent in each quadrant and latency to find the platform, recorded by the software, were later used for statistical analysis.

\subsubsection{Fear conditioning and extinction}

Conditioning was first described by a Russian physiologist I. Pavlov in the beginning of the $20^{\text {th }}$ century. It represents a process of association between an irrelevant (conditioned) stimulus and a relevant (unconditioned) stimulus; as a result, subsequent presentation of the conditioned stimulus produces the unconditioned response in the animal. Cued and contextual fear conditioning is widely used in the phenotyping of mouse mutants (Crawley, 2000).

Conditioning training on day one consists of placing the mouse into the conditioning chamber with metal grid floor and allowing it to explore the novel environment for 2 minutes, during which the baseline freezing is measured. After the 2 minutes of "white noise" the mouse hears a tone (conditioned stimulus), after which it receives a foot shock (unconditioned stimulus). The mouse is then transferred back into home cage. Different foot shock intensity is used for mice of different age; increase in voltage and shock repetitions result in stronger fear memory formation. The tone cue is usually immediately followed by the shock; the strength of cue conditioning depends on the length of the time gap between tone cue and shock.

Fear memory assessment is performed approximately 24 hours afterwards and is divided into context and cue testing. In the context testing the mouse is placed into the conditioning chamber and presented with the white noise. In the cue testing the mouse is placed into a novel chamber, as different as possible from the conditioning chamber in shape, floor texture and odour. It is allowed to explore the chamber for 2 or 3 minutes, after which the tone (cue) is presented. The freezing response in both chambers is usually scored online or offline (from video-recorded sessions) every 5 or 10 seconds for 2-3 minutes. Freezing is a common response to fearful situations and is characterized by the absence of any movement apart from breathing.

In this study the conditioning chamber had the dimensions of $35 \times 20,5 \times 20,5 \mathrm{~cm}$, had a metal grid floor and was usually washed with ethanol. On the first day (training) the mouse was placed into the chamber and presented with white noise for 120 seconds, followed by 30 seconds $10 \mathrm{kHz}$ tone, after which $0,4 \mathrm{~mA}$ foot shock was given for 2 seconds. After a 30 second break the tone and the shock were repeated, and the mouse was returned back into the home cage. 
In the recent memory testing the mouse was placed into the conditioning chamber on the next day, and the freezing was scored online every 5 seconds for 120 seconds, after which the mouse was returned into the home cage. Following a 3 hour break the cue testing was performed in $36 \times 21 \times 35 \mathrm{~cm}$ novel chamber with white walls and Plexiglas floor, usually washed with water. The baseline freezing was scored every 5 seconds for 120 seconds, after which the mouse was presented with the conditioned tone for 120 seconds, during which the freezing was scored.

In the remote memory testing the mouse was placed again into the conditioning chamber 36 days after training. Freezing response was scored as in recent memory testing. Remote memory testing was performed only for the context memory.

The extinction of fear conditioning memory of the context was performed by placing the mouse into the conditioning chamber on several consecutive days approximately at the same time of the day; each time the freezing response was scored online every 5 seconds for 120 seconds, after which the mouse was returned into the home cage.

\subsection{Doxycycline administration}

Doxycycline was administered to mice via the drinking water. $\mathrm{Ca}^{2+}$ and $\mathrm{Mg}^{2+}$ ions efficiently chelate doxycycline and therefore distilled water should be used to avoid unwanted precipitation of doxycycline. The drinking water should also be supplemented with sucrose, since doxycycline has a bitter taste. Furthermore, doxycycline is light sensitive, and the drinking bottles should therefore be wrapped with aluminum foil. For this thesis the drinking water was supplemented with either $100 \mathrm{mg} / 1$ or $25 \mathrm{mg} / 1$ doxycycline and $1 \%$ sucrose and was replaced every week.

\section{Histological analysis of mice}

\subsection{Transcardial perfusion of adult mice}

Mice were deeply anesthetized by injection with sodium-pentobarbitol $(0.25 \mathrm{mg} / \mathrm{g}$ body weight). The thorax was opened and a $25 \mathrm{G}$ needle was inserted into the left ventricle, followed by a cut into the right atrium. The mouse was then perfused with HBSS solution, pre-warmed to RT. The flow rate of the perfusion pump was set to $1-3 \mathrm{ml} /$ minute and the perfusion was allowed to proceed until all blood was replaced by HBSS solution, after which the mouse was perfused with phosphate-buffered 4\% PFA, pH 7.4 for 10-20 minutes. Organs were removed and post fixed for 30 minutes to 2 hours in phosphate-buffered $4 \%$ PFA at $4{ }^{\circ} \mathrm{C}$.

\subsection{Preparation of embryos and early postnatal animals}

Pregnancies were dated according to vaginal plug with the morning of plug appearance taken as embryonic day (E) 0,5. Pregnant females were sacrificed by cervical dislocation, and embryos were dissected and placed in PBS. Chorion and amnion membranes were taken for subsequent genotyping of corresponding embryos. The GFP-expressing embryos were screened for green fluorescence using the inverted fluorescent microscope. Embryos were fixed for 1-2 hours in ice-cold phosphate-buffered 4\% PFA, followed by three washes in PBS, and kept in PBS at $4^{\circ} \mathrm{C}$ until further processing.

Early postnatal (P0-P2) animals were sacrificed by decapitation. Brains were dissected and fixed for 1-4 hours in ice-cold phosphate-buffered 4\% PFA, followed by three washes in PBS, and kept in PBS at $4^{\circ} \mathrm{C}$ until further processing. 


\subsection{Material processing for paraffin embedding and sectioning}

Brains and embryos were rinsed once with water, dehydrated in a series of solutions with rising ethanol concentration and subsequently incubated in xylol and heated paraffin in the paraffin-infiltration machine (MICROM HMP 110). The processed material was embedded into liquid heated paraffin with the help of paraffin-embedding machine (MICROM AP 280), and blocks were allowed to solidify on a cold plate for 30 minutes and then at $4{ }^{\circ} \mathrm{C}$ overnight. $6 \mu \mathrm{m}-$ thick sections were made on a sliding microtome (MICRON HM 400). Sections were pulled from a heated water bath $\left(42^{\circ} \mathrm{C}\right)$ onto HistoBond ${ }^{\circledR}$ glass slides (Marienfeld), which were then allowed to dry overnight at $37^{\circ} \mathrm{C}$ and stored at RT in closed boxes.

\subsection{Material processing for cryosectioning}

Fresh tissue was frozen on dry ice, wrapped in aluminium foil and parafilm and stored at $-80^{\circ} \mathrm{C}$ until sectioning. Sectioning was performed at $-20^{\circ} \mathrm{C}$. For subsequent laser-dissection, fresh $18 \mu \mathrm{m}$ tissue sections were mounted onto PEN membrane frame slides, stained with thionin staining solution and quickly dried under pressure.

Fixed tissue was washed with PBS and cryoprotected by soaking in $30 \%$ sucrose in PBS solution. After sinking, tissue was washed with PBS, dried with Kimberly-Clark wipes and frozen on dry ice.

Stored at $-80^{\circ} \mathrm{C}$ tissue was first allowed to warm up in the cryostate at $-20^{\circ} \mathrm{C}$ for 30 minutes to 1 hour after which it was glued with Tissue Tek onto tissue support plates. 18-20 $\mu \mathrm{m}$ thick sections were transferred onto HistoBond ${ }^{\circledR}$ (Marienfeld) or SuperFrost ${ }^{\circledR P l u s}$ (Menzel) glass slides, post-fixed for 10 minutes in phosphate-buffered 4\% PFA and washed 3 times in PBS. Glass slides were either used immediately for histochemistry or dried for 3-10 hours in a desiccator and stored at $-80^{\circ} \mathrm{C}$ in tightly closed boxes.

\subsection{In situ hybridization}

Cryosections of mouse brains were acetylated in $0,25 \%$ acetic anhydride in triethanolamine solution, washed in PBS and prehybridized for $4-6$ hours in a $5 \times \mathrm{SSC} / 50 \%$ formamide humidified chamber. cRNA probes were diluted to $1-2 \mathrm{ng} / \mu \mathrm{l}$ concentration in the hybridization buffer, and the hybridization samples were applied to the sections, which were then covered with parafilm and incubated overnight at $65^{\circ} \mathrm{C}$ in a $5 \times \mathrm{SSC} / 50 \%$ formamide humidified chamber. The next day slides were washed three times in $2 \mathrm{xSSC}$ at RT and two times in $0,2 \times S S C$ at $68^{\circ} \mathrm{C}$. After washing in maleic acid buffer (Buffer1) slides were blocked in maleic acid buffer supplemented with blocking agent (Buffer2). Anti-DIG antibody (Boehringer) was diluted 1:2000 in Buffer2 and applied to the sections for 1 hour at RT. Slides were washed twice for 30 minutes in Buffer1, equilibrated for 5 minutes in colour development buffer and then incubated at RT in the darkness in colour development buffer supplemented with BCIP and NBT until desired staining level was achieved. Colour development was stopped by transferring the slides into TE buffer. Stained sections were mounted in Kaiser's gelatine.

\subsection{Immunohistochemistry}

Slides with paraffin sections were deparaffinated in xylol and isopropanol and rehydrated in a series of solution with declining ethanol concentration. After distilled water wash the slides were transferred into citrate buffer and heated in a microwave oven for 10 minutes at 650 Watt (antigen retrieval step). Slides were then allowed to cool to RT, washed in Tris-buffer, blocked for 1 hour in blocking buffer and incubated overnight with primary antibody in a humidified chamber at $4{ }^{\circ} \mathrm{C}$. The next day slides were washed 3 times in Tris-buffer supplemented with $2 \%$ milk powder and incubated with the secondary antibody (fluorescent dye-coupled antibody and 
DAPI) for 45 minutes to 1 hour at RT in a humidified chamber. After three more washes slides were rinsed with distilled water and mounted with AquaPolyMount (Polysciences Inc).

Biotin-coupled secondary antibody was detected with the help of streptavidin-HRP complex (LSAB2 ${ }^{\circledR}$ System-HRP) and liquid DAB-substrate complex (Liquid DAB+Substrate; Dako North America) according to manufacturer's protocol.

\subsection{X-Gal staining}

\subsubsection{X-Gal staining of sections}

20-200 $\mu \mathrm{m}$ thick vibratome sections were washed with PBS and incubated in the dark at $37^{\circ} \mathrm{C}$ in the X-Gal staining solution until the desired level of staining was achieved (for different mouse lines time of staining varied from 10 minutes to several hours). Sections were then washed with PBS and mounted onto HistoBond ${ }^{\circledR}$ glass slides with Eukitt mounting medium.

\subsubsection{X-Gal whole-mount staining of mouse embryos}

Fixed E10,5 - E14,5 embryos were washed with PBS and incubated in the dark at $37^{\circ} \mathrm{C}$ in the X-Gal staining solution until the desired level of staining was achieved, washed again with PBS, post-fixed in $4 \%$ PFA overnight at $4^{\circ} \mathrm{C}$ and processed through a series of glycerol/KOH solutions with raising concentration of glycerol and declining concentration of $\mathrm{KOH}$ (see table) for tissue clearing.

\author{
Solution mix (for $10 \mathrm{ml}$ ) \\ $2 \mathrm{ml}$ glycerol \\ $5 \mathrm{ml}$ glycerol \\ $8 \mathrm{ml}$ glycerol \\ $10 \mathrm{ml}$ glycerol
}

$$
\begin{aligned}
& \text { Duration } \\
& 1-4 \text { days } \\
& 1-4 \text { days } \\
& 1-4 \text { days } \\
& 1-4 \text { days }
\end{aligned}
$$

\section{Microarray analysis}

\subsection{Tissue preparation and laser dissection}

NEX null mutant and wildtype mice were sacrificed at 9 weeks of age by cervical dislocation at $1 \mathrm{pm} \pm 1$ hour. Brains were freshly dissected and immediately placed onto dry ice, wrapped in aluminium foil and parafilm and stored at $-80^{\circ} \mathrm{C}$ until sectioning. Serial coronal 20 $\mu \mathrm{m}$ thick brain cryosections were prepared from the left hemisphere in the region containing dorsal hippocampus (bregma -1,7 to -2,0). For subsequent laser-capture the fresh tissue sections were mounted onto PEN-membrane frame slides, stained with thionin staining solution and rapidly dried under a nitrogen beam. Veritas Microdissection System (Arcturus) was used for LCM. Thionin stained CA1 and CA3 regions of the hippocampus were dissected by the UV laser (laser power set to 3,2-4,0) and attached to the CapSure LCM caps with the help of infrared laser. CA1 and CA3 regions from each animal were collected on separate caps. The caps with the attached material were placed onto $0,5 \mathrm{ml}$ Eppendorf tubes containing $100 \mu \mathrm{l}$ RLT buffer, after which the tubes were inverted and stored upside-down at $-80^{\circ} \mathrm{C}$ until RNA isolation.

\subsection{RNA isolation and probe synthesis}

RNA isolation and probe synthesis were performed by a highly qualified technical assistant according to the protocol applied in the laboratory (Rossner et al., 2006). Briefly, on the 
day of RNA isolation and amplification the frozen samples were rapidly thawed at RT, half of each sample was transferred into a fresh tube, and RNA isolation was performed as described above (1.2.6.). The eluted in $100 \mu \mathrm{l}$ RA samples were pooled within groups (same genotype, same duration of exposure to the enriched environment), and RNA was precipitated and subsequently resuspended with T7-tagged dT21V oligonucleotides. Two-round T7 RNA polymerase-mediated RNA amplification and biotin labelling was performed, after which the probe quality was analyzed with the Agilent 2100 Bioanalyzer.

\subsection{Microarray hybridization}

Microarray hybridization was performed in Max-Planck-Institute for biophysical chemistry on GeneChip Fluidics Station 400 (Affymetrix). At least $10 \mu \mathrm{g}$ of labelled cRNA was fragmented by heating the sample to $95^{\circ} \mathrm{C}$ for $35 \mathrm{~min}$ in a volume of $20 \mu$ l containing $40 \mathrm{~mm}$ Tris acetate, $\mathrm{pH}$ 8.1, $100 \mathrm{mM} \mathrm{KOAc}$, and $30 \mathrm{mM} \mathrm{MgOAc}$. Fragmentation was checked by alkaline agarose electrophoresis. Hybridization, washing, staining, and scanning were performed under standard conditions as described by the manufacturer. Mouse 4302.0 gene chips were used that contain over 22,600 probe sets representing transcripts and variants from over 14,000 wellcharacterized mouse genes.

\subsection{Microarray data analysis}

GeneChip data were analyzed using the software packages MAS version 5 (Affymetrix), $\mathrm{R}$ (http://www.r-project.org) and packages available from Bioconductor (http://www.bioconductor.org). Scanned data were first processed with MAS to convert raw image files (.DAT) to probe signal value files (.CEL). Probe signal values were normalized across samples using $\mathrm{R}$ and the affy package (Bioconductor). Three different normalization Methods have been applied to the gene chip data. These were the "robust multi-chip analysis" (RMA), the standard affymetrix normalization "MAS5" and the "variance stabilized normalization" (VSN). All methods are available as packages from Bioconductor. Selection of differentially expressed genes was performed with the limma package (Bioconductor) employing a moderated T-statistics. This approach was applied to all 3 different normalized data sets. The genes which showed a significant differential expression in all different normalized data sets were considered as the most robust differentially expressed genes. To identify significantly differential expression profiles in time-course microarray experiments we used the MaSigPro package (Conesa et. al., Bioinformatics 2006).

The identification of coordinated changes in a priori defined sets of functionally grouped

genes was performed using nonparametric tests and in parallel with the Gene Set Enrichment Analysis (GSEA) approach (Mootha et al., 2003) The GSEA software package was downloaded (http://www.broad.mit.edu/personal/aravind/GSEA/index.html) and used with default parameters (permutations set to 1000) and the selected gene sets as suggested. 


\section{ABBREVIATIONS}

All the abbreviations for the names of genes/proteins and chemical entities were used in accordance with the standard nomenclature.

\begin{tabular}{|c|c|}
\hline $\mathrm{ACC}$ & Anterior cingulate cortex \\
\hline AmpR & Ampicillin resistance \\
\hline $\mathrm{BAC}$ & Bacterial artificial chromosome \\
\hline BDNF & Brain-derived neurotrophic factor \\
\hline bHLH & Basic helix-loop-helix \\
\hline $\mathrm{bp}$ & Base pair \\
\hline CIP & Calf intestinal phosphatase \\
\hline CMV & Cytomegalovirus promoter \\
\hline CNS & Central nervous system \\
\hline $\mathrm{CP}$ & Cortical plate \\
\hline DIG & Digoxygenin \\
\hline DNase & Deoxyribonuclease \\
\hline dNTPs & Deoxyribonucleic acid tri-phosphate \\
\hline DMSO & Dimethyl sulfoxide \\
\hline dox & Doxycycline \\
\hline $\mathrm{E}$ & Embryonic day (mouse development) \\
\hline EDTA & Ethylene diamine tetra acetic acid \\
\hline eGFP & Enhanced green fluorescent protein \\
\hline f.c. & Final concentration \\
\hline floxed & Flanked by loxP sites \\
\hline GABA & Gamma-aminobutyric acid \\
\hline GSEA & Gene set enrichment analysis \\
\hline KanR & Kanamycin resistance \\
\hline $\mathrm{kb}$ & kilo base pairs \\
\hline ko & Knock-out (null mutant) \\
\hline 1 & Litre \\
\hline $\operatorname{lacZ}$ & $\beta$-galactosidase \\
\hline LB & Luria's Broth \\
\hline LCM & Laser-capture microdissection \\
\hline LDS & Lithium dodecyl sulfate \\
\hline LTD & Long-term depression \\
\hline LTP & Long-term potentiation \\
\hline $\mathrm{Mb}$ & Mega base pairs \\
\hline $\mathrm{ml}$ & Millilitre \\
\hline mRNA & Messenger RNA \\
\hline NMDA & N-methyl-D-aspartate \\
\hline OD & Optical density \\
\hline ORF & Open reading frame \\
\hline $\mathrm{P}$ & Postnatal day (mouse development) \\
\hline $\mathrm{pA}$ & Polyadenylation signal \\
\hline PBS & Phosphate-buffered saline \\
\hline PCR & Polymerase chain reaction \\
\hline PFA & Paraformaldehyde \\
\hline PFC & Prefrontal cortex \\
\hline pH & $\mathrm{pH}=-\lg \left[\mathrm{H}^{+}\right]$ \\
\hline PNS & Peripheral nervous system \\
\hline
\end{tabular}


PVDF

QRT-PCR

RNase

RNasin

RT

SAM

SDS

SVZ

TAE

TE

TEA

Tris

UV

$\mathrm{VZ}$

WB

wt

X-Gal
Polyvinylidene fluoride

Quantitative PCR

Ribonuclease

Recombinant RNase inhibitor

Room temperature

Significance analysis of microarrays

Sodium dodecyl sulfate

Subventricular zone

Tris-Acetate-EDTA solution

Tris-EDTA solution

Triethanolamine

Tris(hydroxymethyl)aminomethane

Ultraviolet

Ventricular zone

Western blot

wildtype

5-bromo-4-chloro-3-indolyl- $\beta$-D-galactopyranoside 


\section{REFERENCES}

Abe, K., O. Chisaka, F. Van Roy and M. Takeichi (2004). Stability of dendritic spines and synaptic contacts is controlled by alpha N-catenin. Nat Neurosci 7(4): 357-63.

Abraham, W. C. and A. Huggett (1997). Induction and reversal of long-term potentiation by repeated highfrequency stimulation in rat hippocampal slices. Hippocampus 7(2): 137-45.

Ackermann, M. and A. Matus (2003). Activity-induced targeting of profilin and stabilization of dendritic spine morphology. Nat Neurosci 6(11): 1194-200.

Airaksinen, M. S., J. Eilers, O. Garaschuk, H. Thoenen, A. Konnerth and M. Meyer (1997). Ataxia and altered dendritic calcium signaling in mice carrying a targeted null mutation of the calbindin D28k gene. Proc Natl Acad Sci U S A 94(4): 1488-93.

Angelo, M., F. Plattner and K. P. Giese (2006). Cyclin-dependent kinase 5 in synaptic plasticity, learning and memory. J Neurochem 99(2): 353-70.

Barco, A., C. H. Bailey and E. R. Kandel (2006). Common molecular mechanisms in explicit and implicit memory. J Neurochem 97(6): 1520-33.

Barlow, C. and D. J. Lockhart (2002). DNA arrays and neurobiology--what's new and what's next? Curr Opin Neurobiol 12(5): 554-61.

Bartholoma, A. and K. A. Nave (1994). NEX-1: a novel brain-specific helix-loop-helix protein with autoregulation and sustained expression in mature cortical neurons. Mech Dev 48(3): 217-28.

Berger, J., S. Berger, T. C. Tuoc, M. D'Amelio, F. Cecconi, J. A. Gorski, K. R. Jones, P. Gruss and A. Stoykova (2007). Conditional activation of Pax6 in the developing cortex of transgenic mice causes progenitor apoptosis. Development 134(7): 1311-22.

Bertrand, N., D. S. Castro and F. Guillemot (2002). Proneural genes and the specification of neural cell types. Nat Rev Neurosci 3(7): 517-30.

Bilkei-Gorzo, A., I. Racz, K. Michel, A. Zimmer, D. Klingmuller and A. Zimmer (2004). Behavioral phenotype of pre-proenkephalin-deficient mice on diverse congenic backgrounds. Psychopharmacology (Berl) 176(3-4): 343-52.

Birnboim, H. C. and J. Doly (1979). A rapid alkaline extraction procedure for screening recombinant plasmid DNA. Nucleic Acids Res 7(6): 1513-23.

Bishop, K. M., G. Goudreau and D. D. O'Leary (2000). Regulation of area identity in the mammalian neocortex by Emx2 and Pax6. Science 288(5464): 344-9.

Bliss, T. V. and G. L. Collingridge (1993). A synaptic model of memory: long-term potentiation in the hippocampus. Nature 361(6407): 31-9.

Bliss, T. V. and T. Lomo (1973). Long-lasting potentiation of synaptic transmission in the dentate area of the anaesthetized rabbit following stimulation of the perforant path. $J$ Physiol 232(2): 331-56.

Bontempi, B., C. Laurent-Demir, C. Destrade and R. Jaffard (1999). Time-dependent reorganization of brain circuitry underlying long-term memory storage. Nature 400(6745): 671-5.

Bormuth, I. (in preparation). Neuronal bHLH proteins cooperatively regulate axogenesis.

Bozdagi, O., W. Shan, H. Tanaka, D. L. Benson and G. W. Huntley (2000). Increasing numbers of synaptic puncta during late-phase LTP: N-cadherin is synthesized, recruited to synaptic sites, and required for potentiation. Neuron 28(1): $245-59$.

Bradford, M. M. (1976). A rapid and sensitive method for the quantitation of microgram quantities of protein utilizing the principle of protein-dye binding. Anal Biochem 72: 248-54. 
Brandon, E. P., S. F. Logue, M. R. Adams, M. Qi, S. P. Sullivan, A. M. Matsumoto, D. M. Dorsa, J. M. Wehner, G. S. McKnight and R. L. Idzerda (1998). Defective motor behavior and neural gene expression in RIIbeta-protein kinase A mutant mice. J Neurosci 18(10): 3639-49.

Brockschnieder, D., C. Lappe-Siefke, S. Goebbels, M. R. Boesl, K. A. Nave and D. Riethmacher (2004). Cell depletion due to diphtheria toxin fragment A after Cre-mediated recombination. Mol Cell Biol 24(17): 7636-42.

Brouillette, J. and R. Quirion (2007). Transthyretin: A key gene involved in the maintenance of memory capacities during aging. Neurobiol Aging.

Chae, J. H., G. H. Stein and J. E. Lee (2004). NeuroD: the predicted and the surprising. Mol Cells 18(3): 271-88.

Chevalier-Mariette, C., I. Henry, L. Montfort, S. Capgras, S. Forlani, J. Muschler and J. F. Nicolas (2003). CpG content affects gene silencing in mice: evidence from novel transgenes. Genome Biol 4(9): R53.

Chiaramello, A., T. Neuman, D. R. Peavy and M. X. Zuber (1996). The GAP-43 gene is a direct downstream target of the basic helix-loop-helix transcription factors. J Biol Chem 271(36): 22035-43.

Chien, C. T., C. D. Hsiao, L. Y. Jan and Y. N. Jan (1996). Neuronal type information encoded in the basic-helixloop-helix domain of proneural genes. Proc Natl Acad Sci U S A 93(23): 13239-44.

Chilton, J. K. (2006). Molecular mechanisms of axon guidance. Dev Biol 292(1): 13-24.

Cho, J. H., I. S. Kwon, S. Kim, S. H. Ghil, M. J. Tsai, Y. S. Kim, Y. D. Lee and H. Suh-Kim (2001). Overexpression of BETA2/NeuroD induces neurite outgrowth in F11 neuroblastoma cells. J Neurochem 77(1): 103-9.

Cho, J. H. and M. J. Tsai (2004). The role of BETA2/NeuroD1 in the development of the nervous system. Mol Neurobiol 30(1): 35-47.

Clark, R. E., N. J. Broadbent, S. M. Zola and L. R. Squire (2002). Anterograde amnesia and temporally graded retrograde amnesia for a nonspatial memory task after lesions of hippocampus and subiculum. J Neurosci 22(11): 4663-9.

Copeland, N. G., N. A. Jenkins and D. L. Court (2001). Recombineering: a powerful new tool for mouse functional genomics. Nat Rev Genet 2(10): 769-79.

Crawley, J. N. (2000). What's Wrong With My Mouse?: Behavioral Phenotyping of Transgenic and Knockout Mice Wiley \& Sons. 0471316393

de la Prida, L. M., S. Totterdell, J. Gigg and R. Miles (2006). The subiculum comes of age. Hippocampus 16(11): 916-23.

Debiec, J., J. E. LeDoux and K. Nader (2002). Cellular and systems reconsolidation in the hippocampus. Neuron 36(3): 527-38.

Deisseroth, K., S. Singla, H. Toda, M. Monje, T. D. Palmer and R. C. Malenka (2004). Excitation-neurogenesis coupling in adult neural stem/progenitor cells. Neuron 42(4): 535-52.

Dillon, C. and Y. Goda (2005). The actin cytoskeleton: integrating form and function at the synapse. Annu Rev Neurosci 28: 25-55.

Eichenbaum, H. (2004). Hippocampus: cognitive processes and neural representations that underlie declarative memory. Neuron 44(1): 109-20.

Erzurumlu, R. S. and P. C. Kind (2001). Neural activity: sculptor of 'barrels' in the neocortex. Trends Neurosci 24(10): 589-95.

Fanselow, M. S. (1980). Conditioned and unconditional components of post-shock freezing. Pavlov J Biol Sci 15(4): 177-82.

Fanselow, M. S. (2000). Contextual fear, gestalt memories, and the hippocampus. Behav Brain Res 110(1-2): 73-81. 
Farah, M. H., J. M. Olson, H. B. Sucic, R. I. Hume, S. J. Tapscott and D. L. Turner (2000). Generation of neurons by transient expression of neural bHLH proteins in mammalian cells. Development 127(4): 693-702.

Fleischmann, A., O. Hvalby, V. Jensen, T. Strekalova, C. Zacher, L. E. Layer, A. Kvello, M. Reschke, R. Spanagel, R. Sprengel, E. F. Wagner and P. Gass (2003). Impaired long-term memory and NR2A-type NMDA receptordependent synaptic plasticity in mice lacking c-Fos in the CNS. J Neurosci 23(27): 9116-22.

Floresco, S. B., J. K. Seamans and A. G. Phillips (1997). Selective roles for hippocampal, prefrontal cortical, and ventral striatal circuits in radial-arm maze tasks with or without a delay. J Neurosci 17(5): 1880-90.

Fode, C., G. Gradwohl, X. Morin, A. Dierich, M. LeMeur, C. Goridis and F. Guillemot (1998). The bHLH protein NEUROGENIN 2 is a determination factor for epibranchial placode-derived sensory neurons. Neuron 20(3): 48394.

Foster, T. C. and T. C. Dumas (2001). Mechanism for increased hippocampal synaptic strength following differential experience. J Neurophysiol 85(4): 1377-83.

Foster, T. C., J. Gagne and G. Massicotte (1996). Mechanism of altered synaptic strength due to experience: relation to long-term potentiation. Brain Res 736(1-2): 243-50.

Fox, K. (2002). Anatomical pathways and molecular mechanisms for plasticity in the barrel cortex. Neuroscience 111(4): 799-814.

Frankland, P. W. and B. Bontempi (2005). The organization of recent and remote memories. Nat Rev Neurosci 6(2): 119-30.

Frankland, P. W., B. Bontempi, L. E. Talton, L. Kaczmarek and A. J. Silva (2004). The involvement of the anterior cingulate cortex in remote contextual fear memory. Science 304(5672): 881-3.

Frankland, P. W., H. K. Ding, E. Takahashi, A. Suzuki, S. Kida and A. J. Silva (2006). Stability of recent and remote contextual fear memory. Learn Mem 13(4): 451-7.

Frankland, P. W., C. O'Brien, M. Ohno, A. Kirkwood and A. J. Silva (2001). Alpha-CaMKII-dependent plasticity in the cortex is required for permanent memory. Nature 411(6835): 309-13.

Frey, U. and R. G. Morris (1997). Synaptic tagging and long-term potentiation. Nature 385(6616): 533-6.

Frey, U. and R. G. Morris (1998). Synaptic tagging: implications for late maintenance of hippocampal long-term potentiation. Trends Neurosci 21(5): 181-8.

Giese, K. P., N. B. Fedorov, R. K. Filipkowski and A. J. Silva (1998). Autophosphorylation at Thr286 of the alpha calcium-calmodulin kinase II in LTP and learning. Science 279(5352): 870-3.

Gilbert, S. F. (2006). Developmental Biology, Palgrave Macmillan. 8th rev. ed. 087893250X

Goebbels, S. (2002). Zelltyp-spezifische Expression der Rekombinase Cre im Nervensystem der Maus. PhD: ZMBH Heidelberg University.

Goebbels, S., I. Bormuth, U. Bode, O. Hermanson, M. H. Schwab and K. A. Nave (2006). Genetic targeting of principal neurons in neocortex and hippocampus of NEX-Cre mice. Genesis 44(12): 611-21.

Gorski, J. A., T. Talley, M. Qiu, L. Puelles, J. L. Rubenstein and K. R. Jones (2002). Cortical excitatory neurons and glia, but not GABAergic neurons, are produced in the Emx1-expressing lineage. J Neurosci 22(15): 6309-14.

Gossen, M. and H. Bujard (1992). Tight control of gene expression in mammalian cells by tetracycline-responsive promoters. Proc Natl Acad Sci U S A 89(12): 5547-51.

Gotz, M. and L. Sommer (2005). Cortical development: the art of generating cell diversity. Development 132(15): 3327-32.

Goulding, S. E., N. M. White and A. P. Jarman (2000). cato encodes a basic helix-loop-helix transcription factor implicated in the correct differentiation of Drosophila sense organs. Dev Biol 221(1): 120-31. 
Hamasaki, T., A. Leingartner, T. Ringstedt and D. D. O'Leary (2004). EMX2 regulates sizes and positioning of the primary sensory and motor areas in neocortex by direct specification of cortical progenitors. Neuron 43(3): $359-72$.

Hand, R., D. Bortone, P. Mattar, L. Nguyen, J. I. Heng, S. Guerrier, E. Boutt, E. Peters, A. P. Barnes, C. Parras, C. Schuurmans, F. Guillemot and F. Polleux (2005). Phosphorylation of Neurogenin 2 specifies the migration properties and the dendritic morphology of pyramidal neurons in the neocortex. Neuron 48(1): 45-62.

Hassan, B. A. and H. J. Bellen (2000). Doing the MATH: is the mouse a good model for fly development? Genes Dev 14(15): 1852-65.

Hatten, M. E. (1999). Expansion of CNS precursor pools: a new role for Sonic Hedgehog. Neuron 22(1): 2-3.

Hatten, M. E. and N. Heintz (2005). Large-scale genomic approaches to brain development and circuitry. Annu Rev Neurosci 28: 89-108.

Hayashi, M. L., S. Y. Choi, B. S. Rao, H. Y. Jung, H. K. Lee, D. Zhang, S. Chattarji, A. Kirkwood and S. Tonegawa (2004). Altered cortical synaptic morphology and impaired memory consolidation in forebrain- specific dominantnegative PAK transgenic mice. Neuron 42(5): 773-87.

He, F. and Y. E. Sun (2007). Glial cells more than support cells? Int J Biochem Cell Biol 39(4): 661-5.

Heldt, S. A., G. D. Coover and W. A. Falls (2002). Posttraining but not pretraining lesions of the hippocampus interfere with feature-negative discrimination of fear-potentiated startle. Hippocampus 12(6): 774-86.

Hering, H. and M. Sheng (2003). Activity-dependent redistribution and essential role of cortactin in dendritic spine morphogenesis. J Neurosci 23(37): 11759-69.

Hevner, R. F., R. D. Hodge, R. A. Daza and C. Englund (2006). Transcription factors in glutamatergic neurogenesis: conserved programs in neocortex, cerebellum, and adult hippocampus. Neurosci Res 55(3): 223-33.

Holland, L. L. and J. J. Wagner (1998). Primed facilitation of homosynaptic long-term depression and depotentiation in rat hippocampus. J Neurosci 18(3): 887-94.

Hong, H. K., J. L. Chong, W. Song, E. J. Song, A. A. Jyawook, A. C. Schook, C. H. Ko and J. S. Takahashi (2007). Inducible and reversible Clock gene expression in brain using the tTA system for the study of circadian behavior. PLoS Genet 3(2): e33.

Hu, X. D., Q. Huang, X. Yang and H. Xia (2007). Differential regulation of AMPA receptor trafficking by neurabintargeted synaptic protein phosphatase-1 in synaptic transmission and long-term depression in hippocampus. $J$ Neurosci 27(17): 4674-86.

Impey, S., D. M. Smith, K. Obrietan, R. Donahue, C. Wade and D. R. Storm (1998). Stimulation of cAMP response element (CRE)-mediated transcription during contextual learning. Nat Neurosci 1(7): 595-601.

Ince-Dunn, G., B. J. Hall, S. C. Hu, B. Ripley, R. L. Huganir, J. M. Olson, S. J. Tapscott and A. Ghosh (2006). Regulation of thalamocortical patterning and synaptic maturation by NeuroD2. Neuron 49(5): 683-95.

Inoue, T., M. Hojo, Y. Bessho, Y. Tano, J. E. Lee and R. Kageyama (2002). Math3 and NeuroD regulate amacrine cell fate specification in the retina. Development 129(4): 831-42.

Isiegas, C., A. Park, E. R. Kandel, T. Abel and K. M. Lattal (2006). Transgenic inhibition of neuronal protein kinase A activity facilitates fear extinction. J Neurosci 26(49): 12700-7.

Jan, Y. N. and L. Y. Jan (1993). HLH proteins, fly neurogenesis, and vertebrate myogenesis. Cell 75(5): 827-30.

Jan, Y. N. and L. Y. Jan (1994). Neuronal cell fate specification in Drosophila. Curr Opin Neurobiol 4(1): 8-13.

Jarman, A. P., Y. Grau, L. Y. Jan and Y. N. Jan (1993). atonal is a proneural gene that directs chordotonal organ formation in the Drosophila peripheral nervous system. Cell 73(7): 1307-21.

Jimenez, F. and J. Modolell (1993). Neural fate specification in Drosophila. Curr Opin Genet Dev 3(4): 626-32. 
Johnston, M. V., L. Alemi and K. H. Harum (2003). Learning, memory, and transcription factors. Pediatr Res 53(3): 369-74.

Kandel, E. (2006). Biology of the mind. Newsweek 147(13): 47.

Kandel, E. R. (2001). The molecular biology of memory storage: a dialogue between genes and synapses. Science 294(5544): 1030-8.

Kandel, E. R., J. H. Schwartz and T. M. Jessell (2000). Principles of Neural Science Appleton and Lange. 0838577016

Khoo, S., S. C. Griffen, Y. Xia, R. J. Baer, M. S. German and M. H. Cobb (2003). Regulation of insulin gene transcription by ERK1 and ERK2 in pancreatic beta cells. J Biol Chem 278(35): 32969-77.

Kida, S., S. A. Josselyn, S. P. de Ortiz, J. H. Kogan, I. Chevere, S. Masushige and A. J. Silva (2002). CREB required for the stability of new and reactivated fear memories. Nat Neurosci 5(4): 348-55.

Kieffer, B. L. (1999). Opioids: first lessons from knockout mice. Trends Pharmacol Sci 20(1): 19-26.

Kim, J. (2001). Improvement and establishment of the tTAdependent inducible system in the mouse brain. PhD: ZMBH Heidelberg University.

Kim, S. H., J. H. Warram, A. S. Krolewski and A. Doria (2001). Mutation screening of the neurogenin-3 gene in autosomal dominant diabetes. J Clin Endocrinol Metab 86(5): 2320-2.

Kobayashi, S., Y. Ohashi and S. Ando (2002). Effects of enriched environments with different durations and starting times on learning capacity during aging in rats assessed by a refined procedure of the Hebb-Williams maze task. $J$ Neurosci Res 70(3): 340-6.

Krestel, H. E., M. Mayford, P. H. Seeburg and R. Sprengel (2001). A GFP-equipped bidirectional expression module well suited for monitoring tetracycline-regulated gene expression in mouse. Nucleic Acids Res 29(7): E39.

Kruger, M., T. Schmid, S. Kruger, E. Bober and T. Braun (2006). Functional redundancy of NSCL-1 and NeuroD during development of the petrosal and vestibulocochlear ganglia. Eur J Neurosci 24(6): 1581-90.

Kume, H., K. Maruyama, K. Shinozaki, H. Kuzume and K. Obata (1998). Phosphorylation and spatiotemporal distribution of KW8 (NDRF/NeuroD2), a NeuroD family basic helix-loop-helix protein. Brain Res Mol Brain Res 60(1): 107-14.

LeDoux, J. (1996). Emotional networks and motor control: a fearful view. Prog Brain Res 107: 437-46.

LeDoux, J. E. (1995). Emotion: clues from the brain. Annu Rev Psychol 46: 209-35.

Lee, E. C., D. Yu, J. Martinez de Velasco, L. Tessarollo, D. A. Swing, D. L. Court, N. A. Jenkins and N. G. Copeland (2001). A highly efficient Escherichia coli-based chromosome engineering system adapted for recombinogenic targeting and subcloning of BAC DNA. Genomics 73(1): 56-65.

Lee, E. H., W. L. Hsu, Y. L. Ma, P. J. Lee and C. C. Chao (2003). Enrichment enhances the expression of sgk, a glucocorticoid-induced gene, and facilitates spatial learning through glutamate AMPA receptor mediation. Eur $J$ Neurosci 18(10): 2842-52.

Lee, J. E. (1997). Basic helix-loop-helix genes in neural development. Curr Opin Neurobiol 7(1): 13-20.

Lee, J. E., S. M. Hollenberg, L. Snider, D. L. Turner, N. Lipnick and H. Weintraub (1995). Conversion of Xenopus ectoderm into neurons by NeuroD, a basic helix-loop-helix protein. Science 268(5212): 836-44.

Lee, J. L., B. J. Everitt and K. L. Thomas (2004). Independent cellular processes for hippocampal memory consolidation and reconsolidation. Science 304(5672): 839-43.

Lein, E. S., X. Zhao and F. H. Gage (2004). Defining a molecular atlas of the hippocampus using DNA microarrays and high-throughput in situ hybridization. J Neurosci 24(15): 3879-89. 
Levenson, J. M., S. Choi, S. Y. Lee, Y. A. Cao, H. J. Ahn, K. C. Worley, M. Pizzi, H. C. Liou and J. D. Sweatt (2004). A bioinformatics analysis of memory consolidation reveals involvement of the transcription factor c-rel. $J$ Neurosci 24(16): 3933-43.

Lin, C. H., S. Hansen, Z. Wang, D. R. Storm, S. J. Tapscott and J. M. Olson (2005). The dosage of the neuroD2 transcription factor regulates amygdala development and emotional learning. Proc Natl Acad Sci U S A 102(41): 14877-82.

Lin, C. H., J. Stoeck, A. C. Ravanpay, F. Guillemot, S. J. Tapscott and J. M. Olson (2004). Regulation of neuroD2 expression in mouse brain. Dev Biol 265(1): 234-45.

Lisman, J., H. Schulman and H. Cline (2002). The molecular basis of CaMKII function in synaptic and behavioural memory. Nat Rev Neurosci 3(3): 175-90.

Liu, M., S. J. Pleasure, A. E. Collins, J. L. Noebels, F. J. Naya, M. J. Tsai and D. H. Lowenstein (2000). Loss of BETA2/NeuroD leads to malformation of the dentate gyrus and epilepsy. Proc Natl Acad Sci U S A 97(2): 865-70.

Liu, P., N. A. Jenkins and N. G. Copeland (2003). A highly efficient recombineering-based method for generating conditional knockout mutations. Genome Res 13(3): 476-84.

Lynch, G. S., T. Dunwiddie and V. Gribkoff (1977). Heterosynaptic depression: a postsynaptic correlate of longterm potentiation. Nature 266(5604): 737-9.

Ma, Q. (2006). Transcriptional regulation of neuronal phenotype in mammals. J Physiol 575(Pt 2): 379-87.

Ma, Q., C. Fode, F. Guillemot and D. J. Anderson (1999). Neurogenin1 and neurogenin2 control two distinct waves of neurogenesis in developing dorsal root ganglia. Genes Dev 13(13): 1717-28.

Ma, Q., C. Kintner and D. J. Anderson (1996). Identification of neurogenin, a vertebrate neuronal determination gene. Cell 87(1): 43-52.

Mack, V., N. Burnashev, K. M. Kaiser, A. Rozov, V. Jensen, O. Hvalby, P. H. Seeburg, B. Sakmann and R. Sprengel (2001). Conditional restoration of hippocampal synaptic potentiation in Glur-A-deficient mice. Science 292(5526): 2501-4.

Malecki, M. T., U. S. Jhala, A. Antonellis, L. Fields, A. Doria, T. Orban, M. Saad, J. H. Warram, M. Montminy and A. S. Krolewski (1999). Mutations in NEUROD1 are associated with the development of type 2 diabetes mellitus. Nat Genet 23(3): 323-8.

Malenka, R. C. and M. F. Bear (2004). LTP and LTD: an embarrassment of riches. Neuron 44(1): 5-21.

Mansuy, I. M., M. Mayford, B. Jacob, E. R. Kandel and M. E. Bach (1998). Restricted and regulated overexpression reveals calcineurin as a key component in the transition from short-term to long-term memory. Cell 92(1): 39-49.

Marr, D. (1970). A theory for cerebral neocortex. Proc R Soc Lond B Biol Sci 176(43): 161-234.

Marr, D. (1971). Simple memory: a theory for archicortex. Philos Trans R Soc Lond B Biol Sci 262(841): 23-81.

Martelli, A. M., C. Evangelisti, M. Nyakern and F. A. Manzoli (2006). Nuclear protein kinase C. Biochim Biophys Acta 1761(5-6): 542-51.

Martin, S. J., P. D. Grimwood and R. G. Morris (2000). Synaptic plasticity and memory: an evaluation of the hypothesis. Annu Rev Neurosci 23: 649-711.

Mattson, M. P. (2007). Mitochondrial regulation of neuronal plasticity. Neurochem Res 32(4-5): 707-15.

Maviel, T., T. P. Durkin, F. Menzaghi and B. Bontempi (2004). Sites of neocortical reorganization critical for remote spatial memory. Science 305(5680): 96-9.

Mayford, M., M. E. Bach, Y. Y. Huang, L. Wang, R. D. Hawkins and E. R. Kandel (1996). Control of memory formation through regulated expression of a CaMKII transgene. Science 274(5293): 1678-83. 
McCormick, M. B., R. M. Tamimi, L. Snider, A. Asakura, D. Bergstrom and S. J. Tapscott (1996). NeuroD2 and neuroD3: distinct expression patterns and transcriptional activation potentials within the neuroD gene family. Mol Cell Biol 16(10): 5792-800.

McGaugh, J. L. (2005). Emotional arousal and enhanced amygdala activity: new evidence for the old perseverationconsolidation hypothesis. Learn Mem 12(2): 77-9.

McNaughton, B. L., F. P. Battaglia, O. Jensen, E. I. Moser and M. B. Moser (2006). Path integration and the neural basis of the 'cognitive map'. Nat Rev Neurosci 7(8): 663-78.

Minichiello, L., M. Korte, D. Wolfer, R. Kuhn, K. Unsicker, V. Cestari, C. Rossi-Arnaud, H. P. Lipp, T. Bonhoeffer and R. Klein (1999). Essential role for TrkB receptors in hippocampus-mediated learning. Neuron 24(2): 401-14.

Miyata, T., T. Maeda and J. E. Lee (1999). NeuroD is required for differentiation of the granule cells in the cerebellum and hippocampus. Genes Dev 13(13): 1647-52.

Molteni, R., Z. Ying and F. Gomez-Pinilla (2002). Differential effects of acute and chronic exercise on plasticityrelated genes in the rat hippocampus revealed by microarray. Eur J Neurosci 16(6): 1107-16.

Mootha, V. K., C. M. Lindgren, K. F. Eriksson, A. Subramanian, S. Sihag, J. Lehar, P. Puigserver, E. Carlsson, M. Ridderstrale, E. Laurila, N. Houstis, M. J. Daly, N. Patterson, J. P. Mesirov, T. R. Golub, P. Tamayo, B. Spiegelman, E. S. Lander, J. N. Hirschhorn, D. Altshuler and L. C. Groop (2003). PGC-1alpha-responsive genes involved in oxidative phosphorylation are coordinately downregulated in human diabetes. Nat Genet 34(3): $267-73$.

Morgenstern, B. and W. R. Atchley (1999). Evolution of bHLH transcription factors: modular evolution by domain shuffling? Mol Biol Evol 16(12): 1654-63.

Morris, R. (1984). Developments of a water-maze procedure for studying spatial learning in the rat. $J$ Neurosci Methods 11(1): 47-60.

Morris, R. G. M. (1981). Spatial localization does not depend on the presence of local cues. Learning and motivation 12: 239-260.

Mullis, K., F. Faloona, S. Scharf, R. Saiki, G. Horn and H. Erlich (1986). Specific enzymatic amplification of DNA in vitro: the polymerase chain reaction. Cold Spring Harb Symp Quant Biol 51 Pt 1: 263-73.

Munoz, E. M., M. J. Bailey, M. F. Rath, Q. Shi, F. Morin, S. L. Coon, M. Moller and D. C. Klein (2007). NeuroD1: developmental expression and regulated genes in the rodent pineal gland. J Neurochem 102(3): 887-99.

Murase, S., E. Mosser and E. M. Schuman (2002). Depolarization drives beta-Catenin into neuronal spines promoting changes in synaptic structure and function. Neuron 35(1): 91-105.

Mutoh, H., B. P. Fung, F. J. Naya, M. J. Tsai, J. Nishitani and A. B. Leiter (1997). The basic helix-loop-helix transcription factor BETA2/NeuroD is expressed in mammalian enteroendocrine cells and activates secretin gene expression. Proc Natl Acad Sci U S A 94(8): 3560-4.

Mutoh, H., F. J. Naya, M. J. Tsai and A. B. Leiter (1998). The basic helix-loop-helix protein BETA2 interacts with p300 to coordinate differentiation of secretin-expressing enteroendocrine cells. Genes Dev 12(6): 820-30.

Muyrers, J. P., Y. Zhang, F. Buchholz and A. F. Stewart (2000). RecE/RecT and Redalpha/Redbeta initiate doublestranded break repair by specifically interacting with their respective partners. Genes Dev 14(15): 1971-82.

Nader, K. (2003). Memory traces unbound. Trends Neurosci 26(2): 65-72.

Nader, K., G. E. Schafe and J. E. Le Doux (2000). Fear memories require protein synthesis in the amygdala for reconsolidation after retrieval. Nature 406(6797): 722-6.

Naya, F. J., C. M. Stellrecht and M. J. Tsai (1995). Tissue-specific regulation of the insulin gene by a novel basic helix-loop-helix transcription factor. Genes Dev 9(8): 1009-19.

Nguyen, P. V., T. Abel and E. R. Kandel (1994). Requirement of a critical period of transcription for induction of a late phase of LTP. Science 265(5175): 1104-7. 
Nithianantharajah, J. and A. J. Hannan (2006). Enriched environments, experience-dependent plasticity and disorders of the nervous system. Nat Rev Neurosci 7(9): 697-709.

Oda, H., F. Fushimi, M. Kato, M. Kitagawa, K. Araki, N. Seki and H. Ohkubo (2005). Microarray analysis of the genes induced by tetracycline-regulated expression of NDRF/NeuroD2 in P19 cells. Biochem Biophys Res Commun 335(2): 458-68.

Ohsawa, R., T. Ohtsuka and R. Kageyama (2005). Mash1 and Math3 are required for development of branchiomotor neurons and maintenance of neural progenitors. J Neurosci 25(25): 5857-65.

O'Leary, D. D. and Y. Nakagawa (2002). Patterning centers, regulatory genes and extrinsic mechanisms controlling arealization of the neocortex. Curr Opin Neurobiol 12(1): 14-25.

Olson, J. M., A. Asakura, L. Snider, R. Hawkes, A. Strand, J. Stoeck, A. Hallahan, J. Pritchard and S. J. Tapscott (2001). NeuroD2 is necessary for development and survival of central nervous system neurons. Dev Biol 234(1): $174-87$.

O'Reilly, R. C. and J. W. Rudy (2001). Conjunctive representations in learning and memory: principles of cortical and hippocampal function. Psychol Rev 108(2): 311-45.

Parras, C. M., C. Schuurmans, R. Scardigli, J. Kim, D. J. Anderson and F. Guillemot (2002). Divergent functions of the proneural genes Mash1 and Ngn2 in the specification of neuronal subtype identity. Genes Dev 16(3): 324-38.

Pinaud, R., M. R. Penner, H. A. Robertson and R. W. Currie (2001). Upregulation of the immediate early gene arc in the brains of rats exposed to environmental enrichment: implications for molecular plasticity. Brain Res Mol Brain Res 91(1-2): 50-6.

Pinaud, R., L. A. Tremere, M. R. Penner, F. F. Hess, H. A. Robertson and R. W. Currie (2002). Complexity of sensory environment drives the expression of candidate-plasticity gene, nerve growth factor induced-A. Neuroscience 112(3): 573-82.

Plachez, C. and L. J. Richards (2005). Mechanisms of axon guidance in the developing nervous system. Curr Top Dev Biol 69: 267-346.

Price, D. J., H. Kennedy, C. Dehay, L. Zhou, M. Mercier, Y. Jossin, A. M. Goffinet, F. Tissir, D. Blakey and Z. Molnar (2006). The development of cortical connections. Eur J Neurosci 23(4): 910-20.

Quirk, G. J., G. K. Russo, J. L. Barron and K. Lebron (2000). The role of ventromedial prefrontal cortex in the recovery of extinguished fear. J Neurosci 20(16): 6225-31.

Rampon, C., C. H. Jiang, H. Dong, Y. P. Tang, D. J. Lockhart, P. G. Schultz, J. Z. Tsien and Y. Hu (2000). Effects of environmental enrichment on gene expression in the brain. Proc Natl Acad Sci U S A 97(23): 12880-4.

Ratineau, C., M. W. Petry, H. Mutoh and A. B. Leiter (2002). Cyclin D1 represses the basic helix-loop-helix transcription factor, BETA2/NeuroD. J Biol Chem 277(11): 8847-53.

Remondes, M. and E. M. Schuman (2004). Role for a cortical input to hippocampal area CA1 in the consolidation of a long-term memory. Nature 431(7009): 699-703.

Roberson, E. D., J. D. English, J. P. Adams, J. C. Selcher, C. Kondratick and J. D. Sweatt (1999). The mitogenactivated protein kinase cascade couples PKA and PKC to cAMP response element binding protein phosphorylation in area CA1 of hippocampus. J Neurosci 19(11): 4337-48.

Robertson, A., J. Perea, T. Tolmachova, P. K. Thomas and C. Huxley (2002). Effects of mouse strain, position of integration and tetracycline analogue on the tetracycline conditional system in transgenic mice. Gene 282(1-2): 6574.

Ronnback, A., P. Dahlqvist, P. A. Svensson, M. Jernas, B. Carlsson, L. M. Carlsson and T. Olsson (2005). Gene expression profiling of the rat hippocampus one month after focal cerebral ischemia followed by enriched environment. Neurosci Lett 385(2): 173-8.

Ross, S. E., M. E. Greenberg and C. D. Stiles (2003). Basic helix-loop-helix factors in cortical development. Neuron 39(1): 13-25. 
Rossato, J. I., C. G. Zinn, C. Furini, L. R. Bevilaqua, J. H. Medina, M. Cammarota and I. Izquierdo (2006). A link between the hippocampal and the striatal memory systems of the brain. An Acad Bras Cienc 78(3): 515-23.

Rossner, M. J., J. Hirrlinger, S. P. Wichert, C. Boehm, D. Newrzella, H. Hiemisch, G. Eisenhardt, C. Stuenkel, O. von Ahsen and K. A. Nave (2006). Global transcriptome analysis of genetically identified neurons in the adult cortex. J Neurosci 26(39): 9956-66.

Roth, G. and U. Dicke (2005). Evolution of the brain and intelligence. Trends Cogn Sci 9(5): 250-7.

Sanders, M. J., B. J. Wiltgen and M. S. Fanselow (2003). The place of the hippocampus in fear conditioning. Eur J Pharmacol 463(1-3): 217-23.

Scardigli, R., N. Baumer, P. Gruss, F. Guillemot and I. Le Roux (2003). Direct and concentration-dependent regulation of the proneural gene Neurogenin2 by Pax6. Development 130(14): 3269-81.

Schembri, L., R. Dalibart, F. Tomasello, P. Legembre, F. Ichas and F. De Giorgi (2007). The HA tag is cleaved and loses immunoreactivity during apoptosis. Nat Methods 4(2): 107-8.

Schonig, K., F. Schwenk, K. Rajewsky and H. Bujard (2002). Stringent doxycycline dependent control of CRE recombinase in vivo. Nucleic Acids Res 30(23): e134.

Schrijver, N. C., N. I. Bahr, I. C. Weiss and H. Wurbel (2002). Dissociable effects of isolation rearing and environmental enrichment on exploration, spatial learning and HPA activity in adult rats. Pharmacol Biochem Behav 73(1): 209-24.

Schuurmans, C., O. Armant, M. Nieto, J. M. Stenman, O. Britz, N. Klenin, C. Brown, L. M. Langevin, J. Seibt, H. Tang, J. M. Cunningham, R. Dyck, C. Walsh, K. Campbell, F. Polleux and F. Guillemot (2004). Sequential phases of cortical specification involve Neurogenin-dependent and -independent pathways. Embo J 23(14): 2892-902.

Schuurmans, C. and F. Guillemot (2002). Molecular mechanisms underlying cell fate specification in the developing telencephalon. Curr Opin Neurobiol 12(1): 26-34.

Schwab, M. H., A. Bartholomae, B. Heimrich, D. Feldmeyer, S. Druffel-Augustin, S. Goebbels, F. J. Naya, S. Zhao, M. Frotscher, M. J. Tsai and K. A. Nave (2000). Neuronal basic helix-loop-helix proteins (NEX and BETA2/Neuro D) regulate terminal granule cell differentiation in the hippocampus. J Neurosci 20(10): 3714-24.

Schwab, M. H., S. Druffel-Augustin, P. Gass, M. Jung, M. Klugmann, A. Bartholomae, M. J. Rossner and K. A. Nave (1998). Neuronal basic helix-loop-helix proteins (NEX, neuroD, NDRF): spatiotemporal expression and targeted disruption of the NEX gene in transgenic mice. J Neurosci 18(4): 1408-18.

Scoville, W. B. and B. Milner (1957). Loss of recent memory after bilateral hippocampal lesions. J Neurol Neurosurg Psychiatry 20(1): 11-21.

Seet, B. T., I. Dikic, M. M. Zhou and T. Pawson (2006). Reading protein modifications with interaction domains. Nat Rev Mol Cell Biol 7(7): 473-83.

Shalin, S. C., U. Zirrgiebel, K. J. Honsa, J. P. Julien, F. D. Miller, D. R. Kaplan and J. D. Sweatt (2004). Neuronal MEK is important for normal fear conditioning in mice. $J$ Neurosci Res 75(6): 760-70.

Shevtsova, Z., J. M. Malik, U. Michel, U. Scholl, M. Bahr and S. Kugler (2006). Evaluation of epitope tags for protein detection after in vivo CNS gene transfer. Eur J Neurosci 23(8): 1961-9.

Shimizu, C., C. Akazawa, S. Nakanishi and R. Kageyama (1995). MATH-2, a mammalian helix-loop-helix factor structurally related to the product of Drosophila proneural gene atonal, is specifically expressed in the nervous system. Eur J Biochem 229(1): 239-48.

Skaggs, W. E. and B. L. McNaughton (1996). Replay of neuronal firing sequences in rat hippocampus during sleep following spatial experience. Science 271(5257): 1870-3.

Slezak, M. and F. W. Pfrieger (2003). New roles for astrocytes: regulation of CNS synaptogenesis. Trends Neurosci 26(10): 531-5. 
Smyth, G. K., J. Michaud and H. S. Scott (2005). Use of within-array replicate spots for assessing differential expression in microarray experiments. Bioinformatics 21(9): 2067-75.

Sommer, L., Q. Ma and D. J. Anderson (1996). neurogenins, a novel family of atonal-related bHLH transcription factors, are putative mammalian neuronal determination genes that reveal progenitor cell heterogeneity in the developing CNS and PNS. Mol Cell Neurosci 8(4): 221-41.

Soriano, P. (1999). Generalized lacZ expression with the ROSA26 Cre reporter strain. Nat Genet 21(1): 70-1.

Sotres-Bayon, F., D. E. Bush and J. E. LeDoux (2004). Emotional perseveration: an update on prefrontal-amygdala interactions in fear extinction. Learn Mem 11(5): 525-35.

Sprengel, R. and M. T. Hasan (2007). Tetracycline-controlled genetic switches. Handb Exp Pharmacol(178): 49-72.

Squire, L. R. (1992). Memory and the hippocampus: a synthesis from findings with rats, monkeys, and humans. Psychol Rev 99(2): 195-231.

Squire, L. R. and P. Alvarez (1995). Retrograde amnesia and memory consolidation: a neurobiological perspective. Curr Opin Neurobiol 5(2): 169-77.

Squire, L. R. and P. J. Bayley (2007). The neuroscience of remote memory. Curr Opin Neurobiol 17(2): 185-96.

Squire, L. R., C. E. Stark and R. E. Clark (2004). The medial temporal lobe. Annu Rev Neurosci 27: 279-306.

Squire, L. R. and S. Zola-Morgan (1991). The medial temporal lobe memory system. Science 253(5026): 1380-6.

Stein, T. D. and J. A. Johnson (2002). Lack of neurodegeneration in transgenic mice overexpressing mutant amyloid precursor protein is associated with increased levels of transthyretin and the activation of cell survival pathways. $J$ Neurosci 22(17): 7380-8.

Stoykova, A., D. Treichel, M. Hallonet and P. Gruss (2000). Pax6 modulates the dorsoventral patterning of the mammalian telencephalon. J Neurosci 20(21): 8042-50.

Strekalova, T., B. Zorner, C. Zacher, G. Sadovska, T. Herdegen and P. Gass (2003). Memory retrieval after contextual fear conditioning induces c-Fos and JunB expression in CA1 hippocampus. Genes Brain Behav 2(1): 310.

Subramanian, A., P. Tamayo, V. K. Mootha, S. Mukherjee, B. L. Ebert, M. A. Gillette, A. Paulovich, S. L. Pomeroy, T. R. Golub, E. S. Lander and J. P. Mesirov (2005). Gene set enrichment analysis: a knowledge-based approach for interpreting genome-wide expression profiles. Proc Natl Acad Sci U S A 102(43): 15545-50.

Sur, M. and J. L. Rubenstein (2005). Patterning and plasticity of the cerebral cortex. Science 310(5749): 805-10.

Sutherland, G. R. and B. McNaughton (2000). Memory trace reactivation in hippocampal and neocortical neuronal ensembles. Curr Opin Neurobiol 10(2): 180-6.

Suzuki, A., S. A. Josselyn, P. W. Frankland, S. Masushige, A. J. Silva and S. Kida (2004). Memory reconsolidation and extinction have distinct temporal and biochemical signatures. J Neurosci 24(20): 4787-95.

Takahashi, M. and N. Osumi (2002). Pax6 regulates specification of ventral neurone subtypes in the hindbrain by establishing progenitor domains. Development 129(6): 1327-38.

Takehara, K., S. Kawahara and Y. Kirino (2003). Time-dependent reorganization of the brain components underlying memory retention in trace eyeblink conditioning. $J$ Neurosci 23(30): 9897-905.

Tong, L., H. Shen, V. M. Perreau, R. Balazs and C. W. Cotman (2001). Effects of exercise on gene-expression profile in the rat hippocampus. Neurobiol Dis 8(6): 1046-56.

Tronson, N. C. and J. R. Taylor (2007). Molecular mechanisms of memory reconsolidation. Nat Rev Neurosci 8(4): $262-75$.

Tsai, K. J., S. K. Chen, Y. L. Ma, W. L. Hsu and E. H. Lee (2002). sgk, a primary glucocorticoid-induced gene, facilitates memory consolidation of spatial learning in rats. Proc Natl Acad Sci U S A 99(6): 3990-5. 
Uittenbogaard, M. and A. Chiaramello (2000). Differential expression patterns of the basic helix-loop-helix transcription factors during aging of the murine brain. Neurosci Lett 280(2): 95-8.

Uittenbogaard, M. and A. Chiaramello (2002). Constitutive overexpression of the basic helix-loop-helix Nex1/MATH-2 transcription factor promotes neuronal differentiation of PC12 cells and neurite regeneration. $J$ Neurosci Res 67(2): 235-45.

Uittenbogaard, M. and A. Chiaramello (2004). Expression profiling upon Nex1/MATH-2-mediated neuritogenesis in PC12 cells and its implication in regeneration. J Neurochem 91(6): 1332-43.

Uittenbogaard, M. and A. Chiaramello (2005). The basic helix-loop-helix transcription factor Nex-1/Math-2 promotes neuronal survival of PC12 cells by modulating the dynamic expression of anti-apoptotic and cell cycle regulators. J Neurochem 92(3): 585-96.

Uittenbogaard, M., D. L. Martinka, P. F. Johnson, C. Vinson and A. Chiaramello (2007). 5'UTR of the neurogenic bHLH Nex1/MATH-2/NeuroD6 gene is regulated by two distinct promoters through CRE and C/EBP binding sites. J Neurosci Res 85(1): 1-18.

van Praag, H., G. Kempermann and F. H. Gage (2000). Neural consequences of environmental enrichment. Nat Rev Neurosci 1(3): 191-8.

von Hertzen, L. S. and K. P. Giese (2005). Memory reconsolidation engages only a subset of immediate-early genes induced during consolidation. J Neurosci 25(8): 1935-42.

Vyssotski, A. L., G. Dell'Omo, Poletaeva, II, D. L. Vyssotsk, L. Minichiello, R. Klein, D. P. Wolfer and H. P. Lipp (2002). Long-term monitoring of hippocampus-dependent behavior in naturalistic settings: mutant mice lacking neurotrophin receptor TrkB in the forebrain show spatial learning but impaired behavioral flexibility. Hippocampus 12(1): 27-38.

Wang, X., V. Korzh and Z. Gong (2002). The functional specificity of NeuroD is defined by a single amino acid residue (N11) in the basic domain. FEBS Lett 520(1-3): 139-44.

Wehr, M. C., R. Laage, U. Bolz, T. M. Fischer, S. Grunewald, S. Scheek, A. Bach, K. A. Nave and M. J. Rossner (2006). Monitoring regulated protein-protein interactions using split TEV. Nat Methods 3(12): 985-93.

Weintraub, H. (1993). The MyoD family and myogenesis: redundancy, networks, and thresholds. Cell 75(7): 12414.

Whitlock, J. R., A. J. Heynen, M. G. Shuler and M. F. Bear (2006). Learning induces long-term potentiation in the hippocampus. Science 313(5790): 1093-7.

Wilson, M. A. (2002). Hippocampal memory formation, plasticity, and the role of sleep. Neurobiol Learn Mem 78(3): 565-9.

Winder, D. G., I. M. Mansuy, M. Osman, T. M. Moallem and E. R. Kandel (1998). Genetic and pharmacological evidence for a novel, intermediate phase of long-term potentiation suppressed by calcineurin. Cell 92(1): 25-37.

Wolpert, L., T. M. Jessel and P. Lawrence (2006). Principles of development, Oxford University Press. 3rd ed. 019927536X

Wu, S. X., S. Goebbels, K. Nakamura, K. Nakamura, K. Kometani, N. Minato, T. Kaneko, K. A. Nave and N. Tamamaki (2005). Pyramidal neurons of upper cortical layers generated by NEX-positive progenitor cells in the subventricular zone. Proc Natl Acad Sci U S A 102(47): 17172-7.

Yasuda, M. and M. R. Mayford (2006). CaMKII activation in the entorhinal cortex disrupts previously encoded spatial memory. Neuron 50(2): 309-18.

Yasunami, M., K. Suzuki, H. Maruyama, H. Kawakami, Y. Nagai, M. Hagiwara and H. Ohkubo (1996). Molecular cloning and characterization of a cDNA encoding a novel basic helix-loop-helix protein structurally related to Neuro-D/BHF1. Biochem Biophys Res Commun 220(3): 754-8.

Yu, D., H. M. Ellis, E. C. Lee, N. A. Jenkins, N. G. Copeland and D. L. Court (2000). An efficient recombination system for chromosome engineering in Escherichia coli. Proc Natl Acad Sci U S A 97(11): 5978-83. 
Zhang, Y., F. Buchholz, J. P. Muyrers and A. F. Stewart (1998). A new logic for DNA engineering using recombination in Escherichia coli. Nat Genet 20(2): 123-8. 


\section{APPENDIX}

The Tet-off system has been successfully applied to the analysis of brain function (Mayford et al., 1996; Mack et al., 2001; Yasuda and Mayford., 2006). The system requires two components: the tetracycline-controlled transactivator (tTA) and a target gene under the control of a hybrid promoter that combines sequences of the human cytomegalovirus promoter (CMV) and tet operator (tetO) of E. coli (Gossen and Bujard, 1992). In the absence of doxycycline (Dox, a tetracycline derivative), tTA binds to tetO and activates transcription of the target gene. In contrast, in the presence of Dox, tTA dissociates from tetO, resulting in termination of transcription of the target gene. This inducible gene expression system allows alterations of transcription in intact animals and therefore provides a valuable tool for gain-of-function and dominant-negative mutation studies in the mouse. Control of gene expression at specifically chosen time points is often required to uncouple the possible developmental effects from the adult function of the gene-of-interest and would facilitate studies of neuronal transcription factors in the context of behavioural analysis.

The original goal of my $\mathrm{PhD}$ thesis was to create two transgenic mouse lines, in which the expression of wildtype (wt) or dominant-negative (dn) forms of the neuronal bHLH transcription factor NeuroD could be modulated by doxycycline administration to adult mice. These mouse lines would have been used to assess the role of NeuroD in higher cognitive functions of adult and senile mice in both gain- and loss-of-function approaches.

In the first gain-of-function approach, a Flag-tag (aminoacid sequence: MDYKDDDDK) was fused to the N-terminus of wildtype NeuroD, and the cassette was cloned into a bidirectional Tet-operator construct, harbouring a $\beta$-galactosidase reporter gene. Functional integrity of this transgenic construct was assessed by X-gal staining of transfected cells, immunocytochemistry and Western blotting against Flag-tag (Suppl. Fig. 1). For this and following transgene functionality assessments in vitro a PC12-TetOff cell line with constitutive tTA expression was used. 


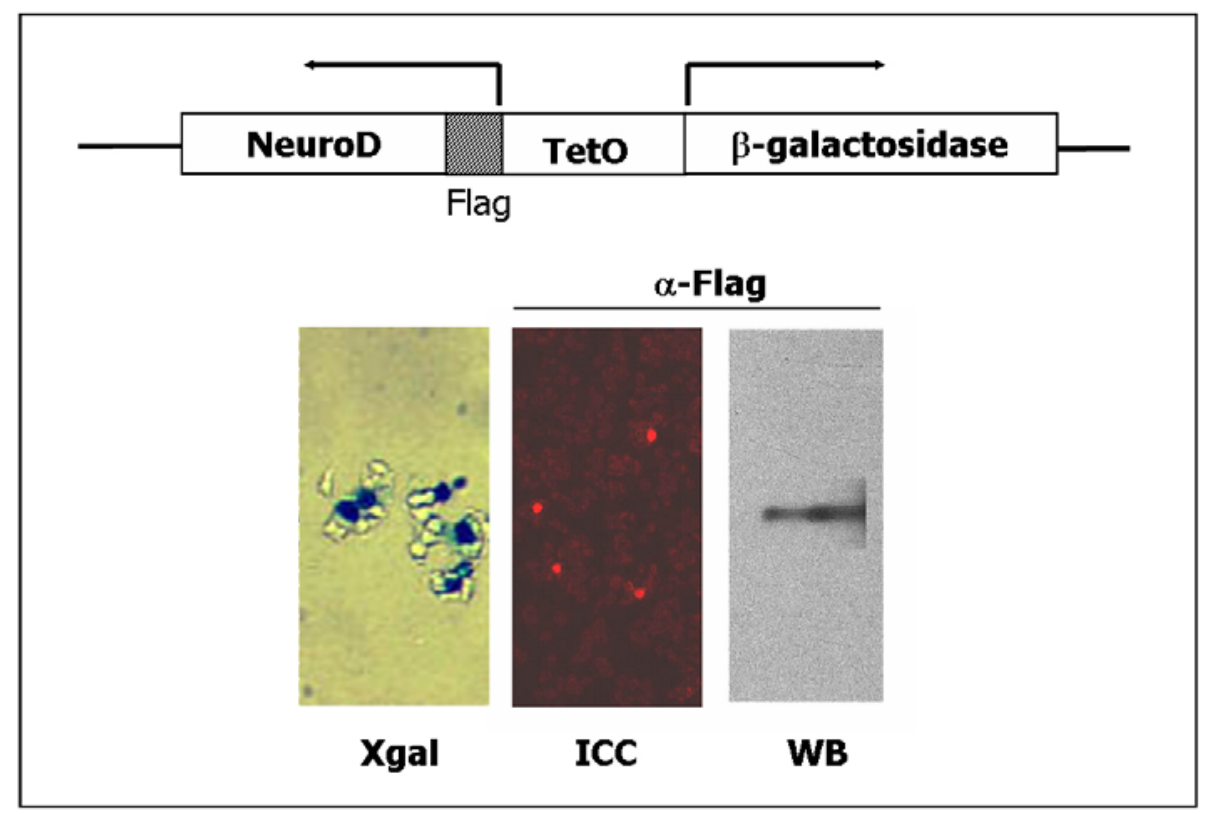

Suppl. Fig. 1 NeuroD gain-of-function transgenic construct 1: in vitro activity analysis

PC12 TetOff cells were transfected with the bidirectional tetO transgenic construct (above), and functional analysis was performed by Xgal histochemistry (Xgal), immunocytochemistry against Flag-tag (ICC) and Western blotting (WB) (below).

In the second gain-of-function approach, a 3xFlag-tag (aminoacid sequence: MDYKDHDGDYKDHDIDYKDDDDKLAAANSSIDLISTSLYKKAGS) was fused to the Nterminus of wildtype NeuroD, and the cassette was cloned into a modified bi-directional tetO vector. The modified vector (TOBi-TRUE) harboured splice donor and acceptor sites on both sides of the tetO promoter and an enhanced green fluorescent protein (eGFP) ORF instead of the $\beta$-galactosidase reporter. Functional integrity of the new transgenic construct was tested by Western blotting and immunocytochemistry against Flag-tag (Suppl. Fig. 2). Doxycycline treatment of transfected PC12 TetOff cells showed that reporter gene expression was efficiently shut down by adding low doses of doxycycline to the culture medium. 


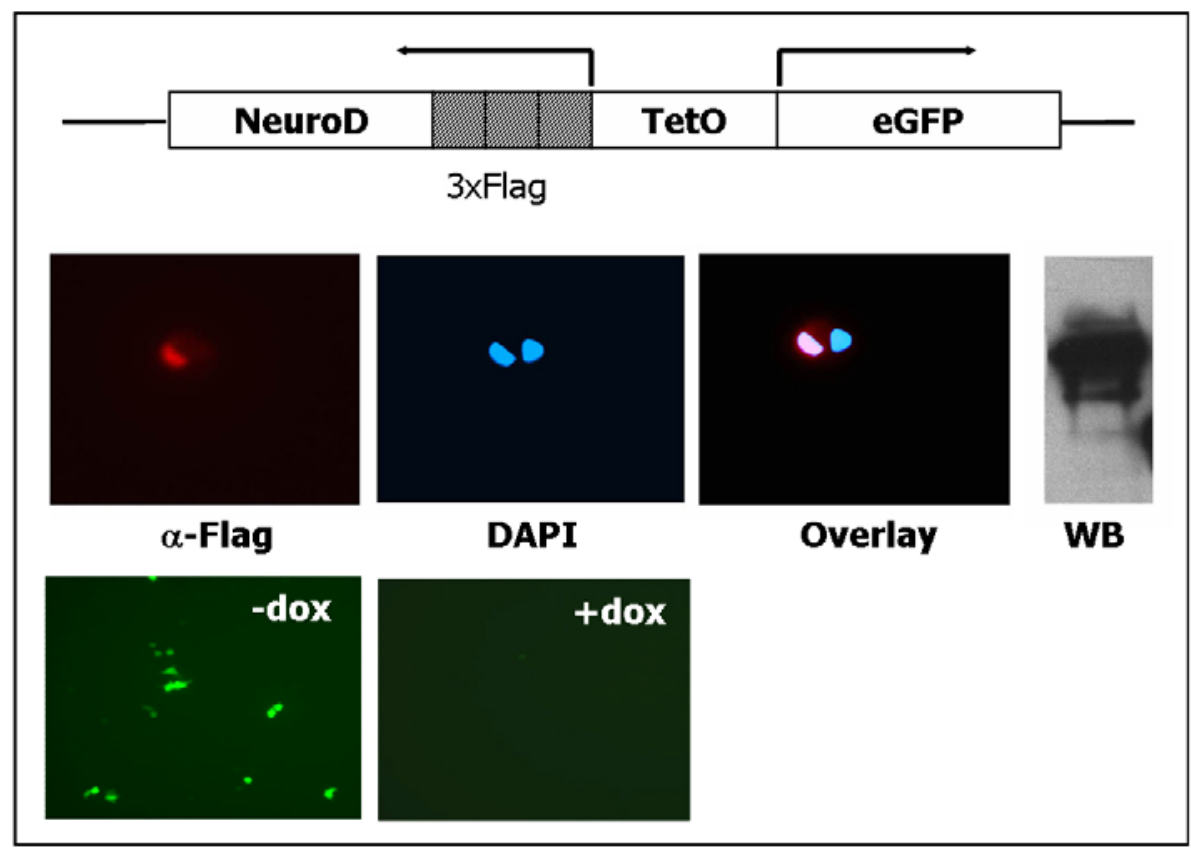

Suppl. Fig. 2 NeuroD gain-of-function transgenic construct 2: in vitro activity analysis

PC12 TetOff cells were transfected with the bidirectional tetO transgenic construct (above), and functional analysis was performed by immunocytochemistry against Flag-tag and Western blotting (WB) (middle panel). eGFP expression was efficiently suppressed by addition of doxycycline to the medium (below).

(DAPI - nuclear stain; eGFP - enhanced green fluorescent protein; WB - Western blot)

For the loss-of-function approach, a dominant-negative form of NeuroD (dnNeuroD) was designed, in which the transcriptional activation domain of NeuroD was replaced by transcriptional repression domain. The KRAB domain of NK10 has been shown to confer potent transcriptional repression in mammalian cells (Thiel et al., 2001). Various versions of fusions between bHLH domain-harbouring part of NeuroD and KRAB domain of NK10 (see Suppl. Fig. 3) were tested in cell culture for their ability to repress luciferase reporter gene expression in PC12 cells, for which a modified version of pTK-luci reporter plasmid was used. The modified reporter plasmid contained a 4xE-box cis-element (tgCAGATGgaagcCATCTGcgtg CAGATGgaagcCATCTGcg) that has previously been shown to drive wtNeuroD-mediated transcriptional activation (data not shown). Of all the chimaeric proteins tested, only the fusion of KRAB domain to the N-terminus of NeuroD truncated version (first 155 aminoacids; see constructs 8 and 9 in Suppl. Fig. 3) efficiently repressed luciferase activity in PC12 cells (Suppl. Fig. 4A). It is, however, unclear whether the positioning of KRAB domain at the N-terminus rather than at the C-terminus of the chimaeric protein, or the deletion of extended homology helix in the NeuroD part, or both account for the transcriptional repression potential. 

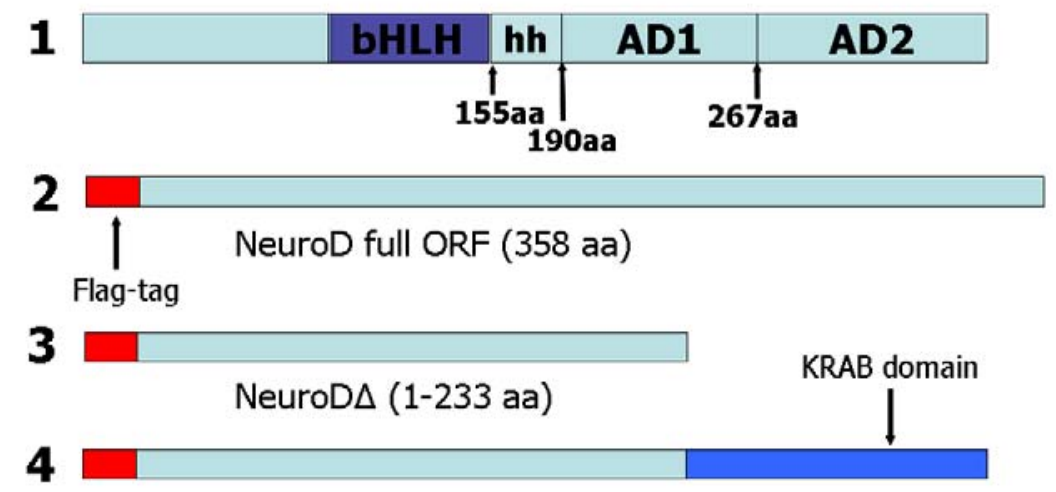

NeuroD $\Delta(1-233$ aa) fused with RD

5 NeuroDA (1-192 aa) fused with RD

6 NeuroDA (1-192 aa) fused with RD trough flexible linker SGGGGS

7 NeuroDA (1-192 aa) fused with RD trough helical linker ASPSNPGASNGS

8

NeuroD $\Delta$ (1-155 aa) fused with RD at N-terminus

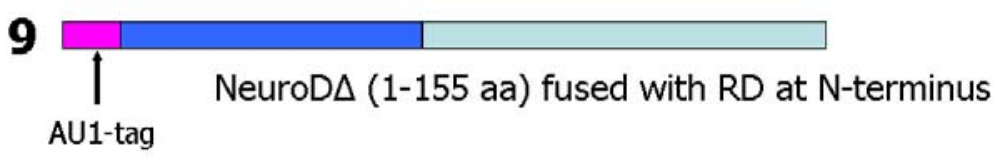

Suppl. Fig. 3 Schematic structure of various dominant-negative forms of NeuroD.

1 - domain structure of wildtype NeuroD protein.

2 - Flag tag was fused to the N-terminus of NeuroD ORF; this construct was later used as wtNeuroD.

$3-233$ aminoacid truncated version of NeuroD, lacking most of the transcriptional activation domains.

4 - Same as 3, fused with KRAB repressor domain at C-terminus.

5, 6, 7 - KRAB domain was fused at the C-terminus to a 192 aminoacid truncated version of NeuroD (completely lacking the transcriptional activation domains) directly $(5)$ or via a linker $(6,7)$.

8 - KRAB repressor domain was fused to a 155 aminoacid truncated version of NeuroD (lacking the transcriptional activation domains and the extended homology helix) at the $\mathrm{N}$-terminus.

9 - Same as 8 , harbouring an AU1-tag at the N-terminus.

(aa - aminoacid; AD1, AD2 - transcriptional activation domains 1 and 2; bHLH - basic helix-loop-helix domain; hh - extended homology helix; $\mathrm{RD}$ - transcriptional repression domain)

The chosen dnNeuroD version (8 and 9 in Suppl.Fig. 3) was further tested in cell culture for its ability to interfere with wildtype NeuroD protein function. For this, PC12 cells were transfected with different ratios of wtNeuroD: dnNeuroD expression vectors, and the reporter activity was measured. dnNeuroD efficiently repressed NeuroD-dependent transcriptional activation at the vectors ratio of 1:1 (Suppl. Fig. 4B), and this interference was DNA-binding dependent (Suppl. Fig. 4C). 

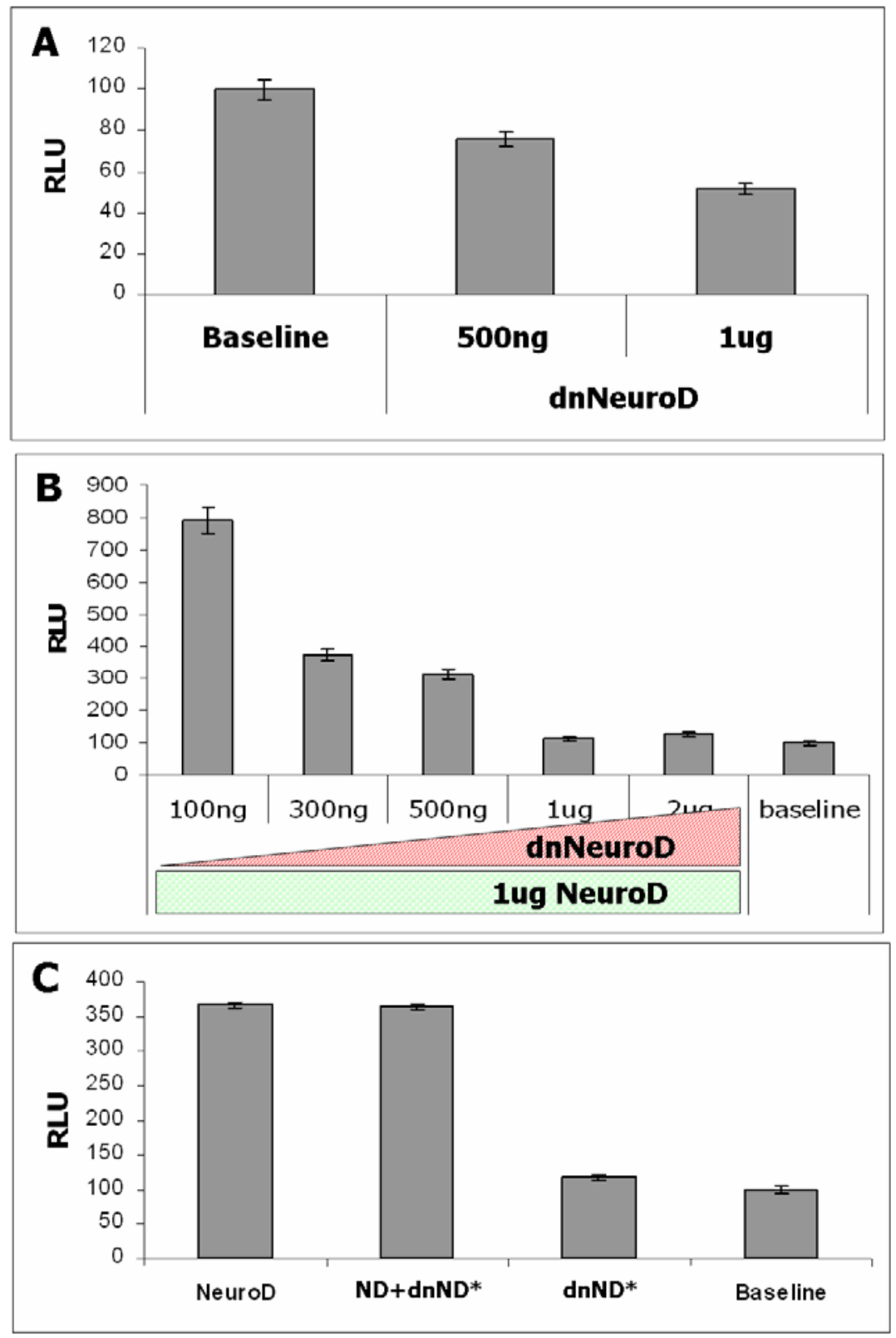

\section{Suppl. Fig. 4 dnNeuroD represses transcription and interferes with NeuroD-mediated transcriptional activation in vitro.}

Expression of dnNeuroD (construct 9 in Suppl. Fig. 3) in PC12 cells induced repression of luciferase reporter activity compared to baseline (A) and interfered with wtNeuroD-induced activation of reporter gene expression in a dosage-dependent manner (B). When a mutation was introduced into the basic (DNA-binding) domain of dnNeuroD, its ability to repress the reporter gene transcription was lost (C).

The reporter construct used for this assay contained luciferase gene under the control of TK promoter, supplemented with 4 E-box sequences (NeuroD-responsive cis-elements).

(baseline - baseline luciferase activity in PC12 cells, transfected only with reporter plasmids; dnND* - dnNeuroD with a mutation in the basic domain; ND- NeuroD; RLU - relative luciferase activity units)

This dnNeuroD chimaeric cassette was used to generate a tetracycline-dependent dominant negative transgenic construct. AU1-tag (aminoacid sequence: DTYRYI) was added by PCR to the N-terminus of dnNeuroD, after which it was cloned into the TOBi-TRUE construct. 
The functional integrity of the construct was checked in PC12 cells (Suppl. Fig. 5) as well as in transiently transfected primary mouse cortical neurons (data not shown).

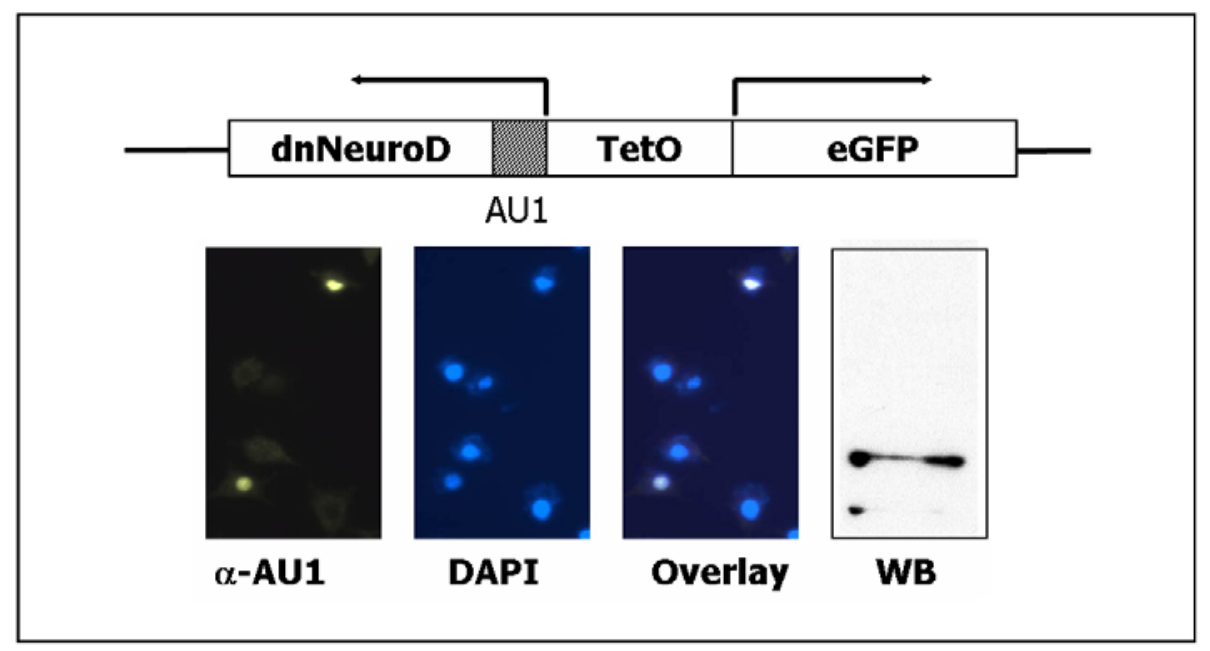

Suppl. Fig. 5 dnNeuroD transgenic construct: in vitro activity analysis

PC12 TetOff cells were transfected with the bidirectional tetO transgenic construct (above), and functional analysis was performed by immunocytochemistry against AU1-tag and Western blotting (below).

(DAPI - nuclear stain; eGFP - enhanced green fluorescent protein; WB - Western blot)

Multiple rounds of transgene injection into C57B16 and FVB oocytes were performed. The ratio of PCR-positive founder mice was within 5-30\% for each injection. However, none of the founder lines displayed the expected pattern of reporter gene expression when bred with the CaMKII-tTA mouse line (data not shown). The table below gives an overview of the number of transgenic founders screened for all three constructs:

\begin{tabular}{|l|c|c|}
\hline Transgenic construct & Number of founders & Reporter expression \\
\hline $\begin{array}{l}\text { wtNeuroD overexpression 1 } \\
\text { (see Suppl. Fig. 1) }\end{array}$ & $12+8$ & NO* \\
\hline $\begin{array}{l}\text { wtNeuroD overexpression } 2 \\
\text { (see Suppl. Fig. 2) }\end{array}$ & 12 & NO \\
\hline $\begin{array}{l}\text { dnNeuroD overexpression 1 } \\
\text { (see Suppl. Fig. 5) }\end{array}$ & 6 & $1 * *$ \\
\hline $\begin{array}{l}\text { NEX-htTA (see Results } \\
\text { chapter, Fig. 20-24) }\end{array}$ & 13 & NO \\
\hline
\end{tabular}

* - one line displayed lacz activity, but it was restricted to CA3 region of the hippocampus and the cerebellum, i.e. did not fully recapitulate the CaMKII expression pattern.

** - as shown in Fig. 24 and Tab. 6, postnatal reporter activity is restricted to hippocampus and entorhinal cortex, i.e. does not fully recapitulate NEX expression pattern.

Altogether, 51 founders have been screened for transgene activity in the adult brain (38 for reporter lines and 13 for NEX-htTA line), of which only two demonstrated X-gal staining. In these two the pattern of $\beta$-galactosidase activity was restricted to only a subset of brain areas and 
did not fully follow the expected pattern (see results section, Fig. 24; for reporter lines data not shown).

Several transgenic mouse lines that employ tet-dependent gene expression in the brain have been reported (for review, see Sprengel and Hasan, 2007). The reason for the poor outcome in our approach could potentially be the effect of the transgenic construct integration site, or sequence-dependent silencing of the transgene (due to the parts of bacterial DNA elements they contained), or both. All transgenic constructs used did not exceed the size of $10 \mathrm{~kb}$, which can be a reason for integration site-mediated silencing. On the other hand, the current knowledge of tetsystem-based transgene efficiency does not provide the information about the number of founders one has to screen before obtaining a functional mouse line. The results presented in this appendix were obtained for 3 different transgenic constructs and represent full-time research work during a period of 2 years and 5 months. Altogether, the data presented here suggest that some advances in brain-specific application of the tet-system as well as relevant modifications of the currently used transgenic approaches are required to develop a reliable system of tetracycline-regulated gene expression in the adult mouse brain. 


\section{CURRICULUM VITAE}

Personal data

Address:

Date and place of birth:

Citizenship:

$1984-1987$

$1987-1994$

$1994-1998$

$2000-2002$

$2002-2007$

\section{Olga Mikhailova}

Böttingerstr. 5

37073 Göttingen

$2^{\text {nd }}$ of February, 1978; Brest, Belarus

Russian Federation

Primary school, Lahdenpohya (Russia), Brest (Belarus), StPetersburg (Russia)

Secondary school, St-Petersburg (Russia)

St-Petersburg State University, Russia

Faculty of Biology and Soil Sciences

Diploma (B.Sc.), Topic:

"Axes formation in the early development of a free-living sea nematode Enoplus brevis"

Molecular Biology Graduate Programme, Göttingen, Germany Diploma (M.Sc.), Topic:

"Knockdown of cytoplasmic dynein heavy chain $\mathbf{2}$ in mouse zygotes by means of RNA interference"

PhD, Topic:

"The role of bHLH transcription factor NEX in neuronal differentiation and experience-dependent plasticity"

Dept. Neurogenetics, Prof. Dr. Klaus-Armin Nave,

Max-Planck-Institute of Experimental Medicine, Göttingen

\section{PUBLICATIONS}

- Mikhailova et al. (in preparation) Uncoupling of neuronal development from functional plasticity by mutation in the bHLH transcription factor gene.

- Mikhailova et al., (in preparation) Doxycycline-regulated gene expression in embryonic forebrain pyramidal neurons. 